\title{
MODELING SHEET-FLOW SAND TRANSPORT UNDER PROGRESSIVE SURFACE WAVES
}

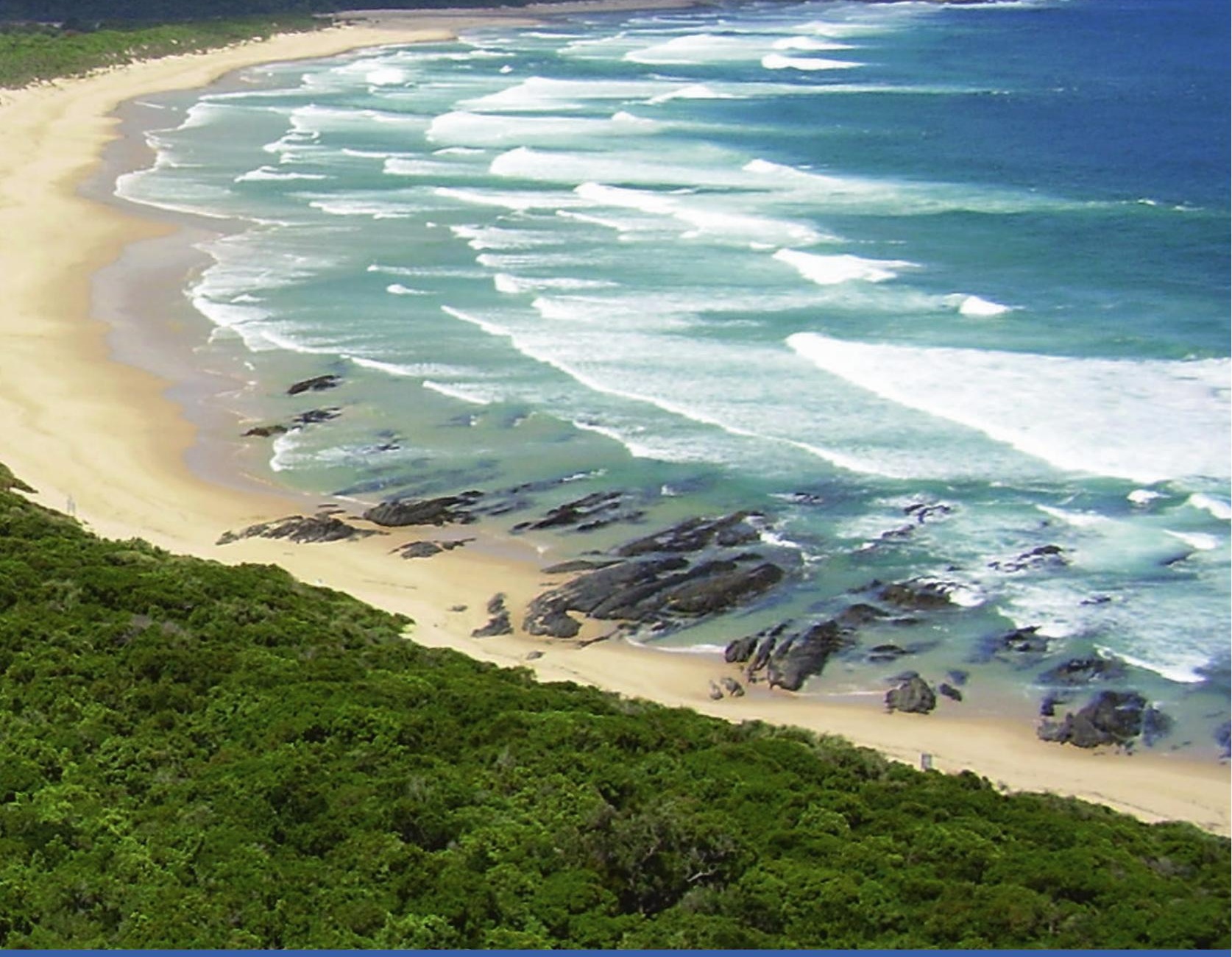


MODELING SHEET-FLOW SAND TRANSPORT

UNDER PROGRESSIVE SURFACE WAVES 
Promotion committee:

Prof. dr. F. Eising

Prof. dr. S.J.M.H. Hulscher

Dr. ir. J.S. Ribberink

Dr. ir. R.E. Uittenbogaard

Prof. A.G. Davies, MSc. PhD.

Prof. dr. ir. L. van Rijn

Prof. dr. ir. Z.B. Wang

Prof. dr. rer.-nat. S. Luding

Dr. ir. C.M. Dohmen-Janssen
University of Twente, chairman and secretary

University of Twente, supervisor

University of Twente, assistant supervisor

Deltares, Delft

Bangor University, UK

Utrecht University

Delft University of Technology

University of Twente

University of Twente

ISBN 978-90-365-3504-5

DOI $10.3990 / 1.9789036535045$

URL http://dx.doi.org/10.3990/1.9789036535045

Copyright (C) 2013 by W.M. Kranenburg, Enschede, The Netherlands

Cover: Nature's Valley, Western Cape, South-Africa; picture by W.M. Kranenburg

Printed by Gildeprint Drukkerijen, Enschede, The Netherlands 


\title{
MODELING SHEET-FLOW SAND TRANSPORT \\ UNDER PROGRESSIVE SURFACE WAVES
}

\section{PROEFSCHRIFT}

\author{
ter verkrijging van \\ de graad van doctor aan de Universiteit Twente, \\ op gezag van de rector magnificus, \\ prof. dr. H. Brinksma, \\ volgens besluit van het College voor Promoties \\ in het openbaar te verdedigen op \\ vrijdag 15 februari 2013 om 14:45 uur
}

door

Wouter Michiel Kranenburg

geboren op 6 juni 1981

te Zoetermeer 
Dit proefschrift is goedgekeurd door:

prof. dr. S.J.M.H. Hulscher

promotor

dr. ir. J.S. Ribberink

assistent-promotor 




\section{CONTENTS}

$\begin{array}{ll}\text { Contents } & 7\end{array}$

$\begin{array}{lll}\text { Summary } & 11\end{array}$

Samenvatting $\quad 13$

1 Introduction $\quad 15$

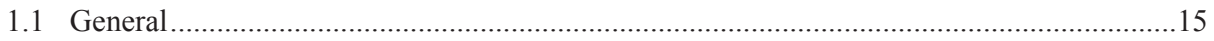

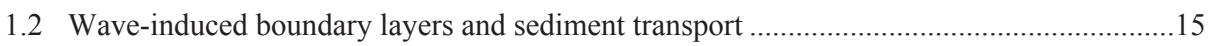

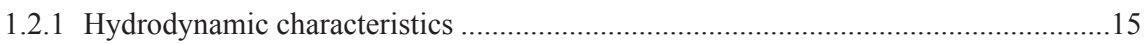

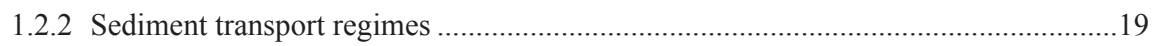

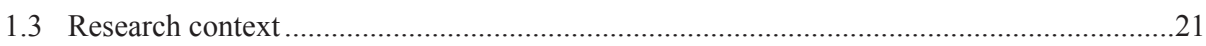

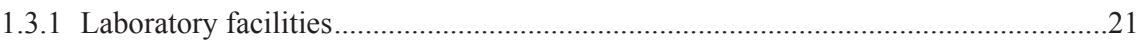

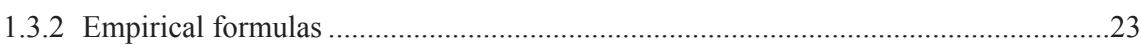

1.3.3 Process-based intra wave boundary layer models .....................................................23

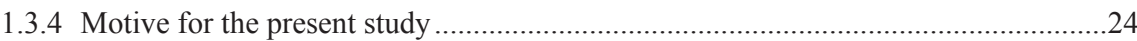

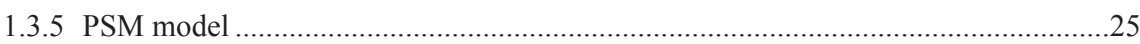

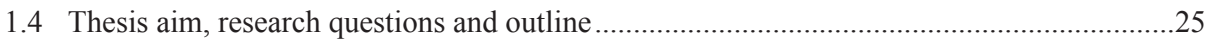

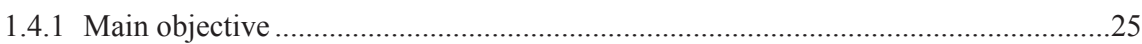

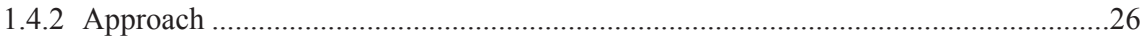

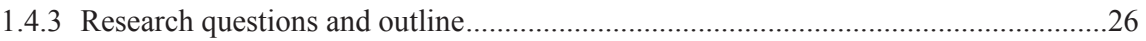

2 Net currents in the wave bottom boundary layer: on wave shape streaming and progressive wave streaming $\quad 29$

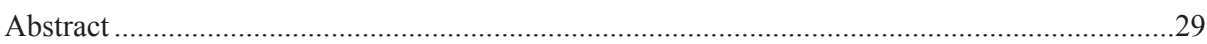

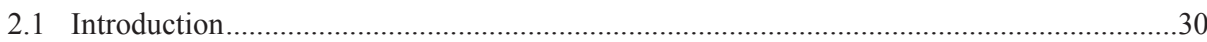

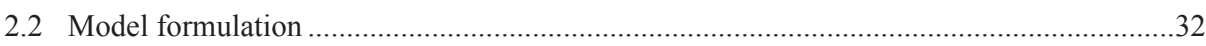

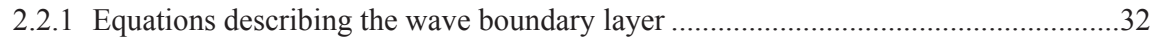

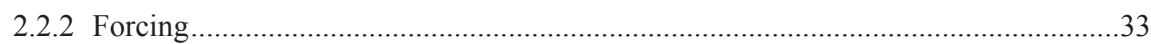

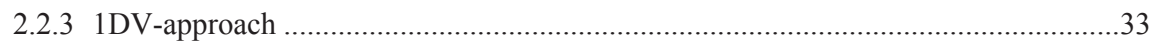

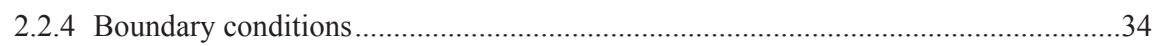

2.2.5 Relation to other numerical boundary layer models .....................................................

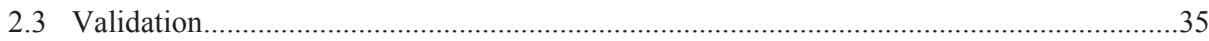

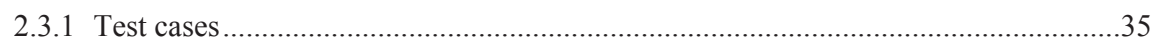

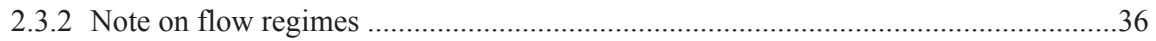

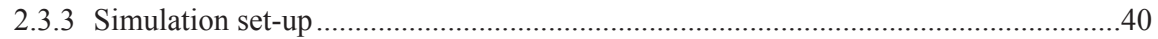

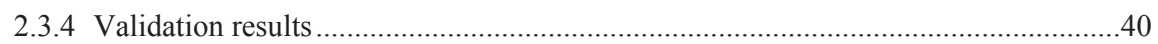

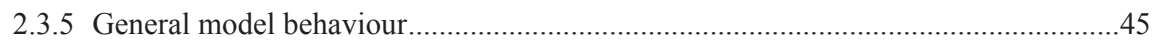

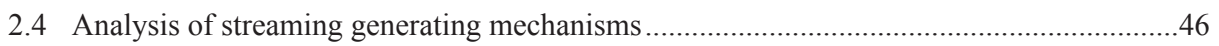

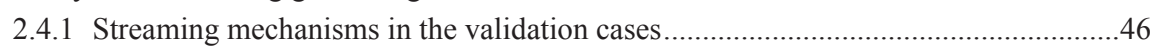

2.4.2 Influence of changing wave and bed conditions.....................................................47 
2.4.3 Effects of a mean pressure gradient on current and stress ...................................... 52

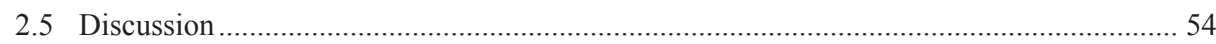

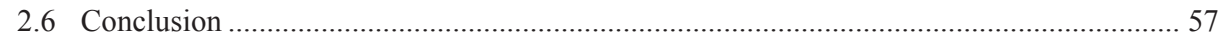

3 Sand transport beneath waves: the role of progressive wave streaming and other free surface effects $\quad 59$

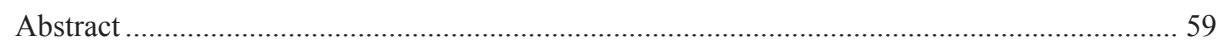

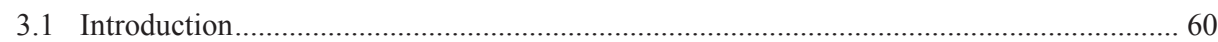

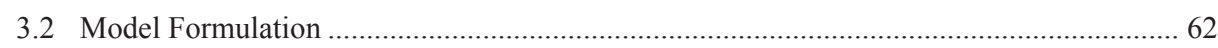

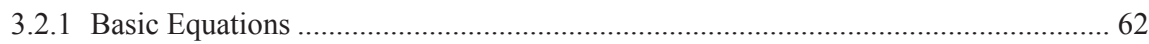

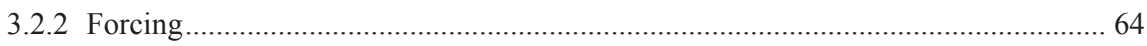

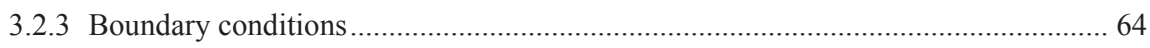

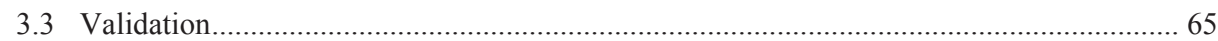

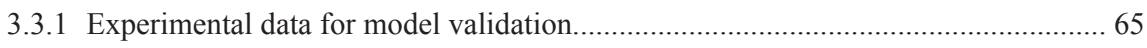

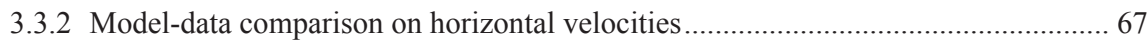

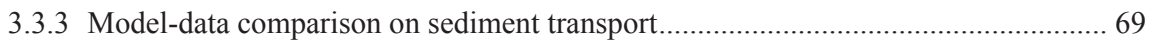

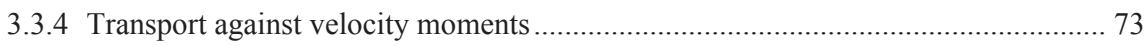

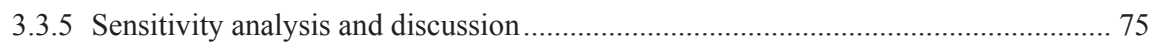

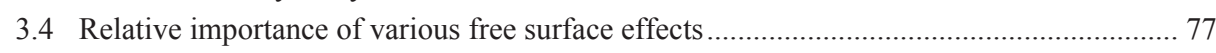

3.4.1 Compensation of mass transport in closed tunnels and flumes ................................ 77

3.4.2 Advection processes: illustration for sinusoidal waves .......................................... 78

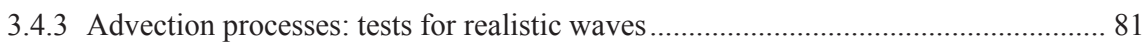

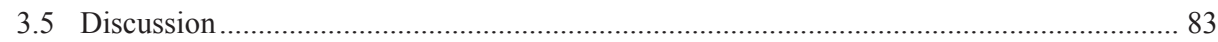

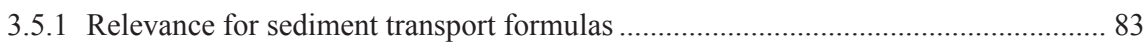

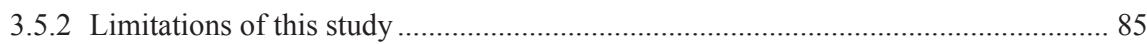

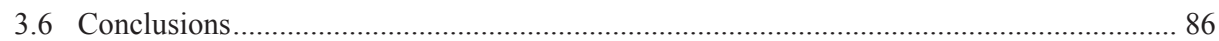

4 Sheet-flow beneath waves: erosion depths and sediment fluxes and their dependence on $\begin{array}{ll}\text { grain size and streaming } & 89\end{array}$

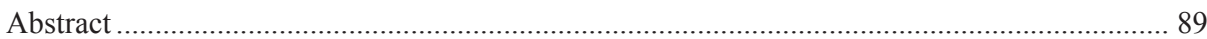

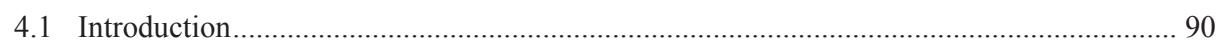

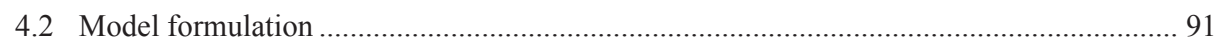

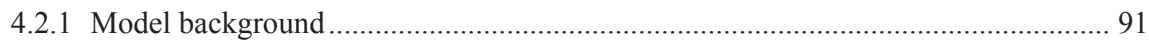

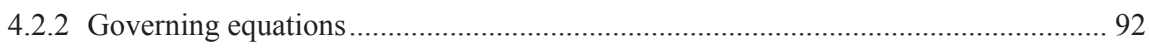

4.2.3 Closures for the fluid and particle stresses ............................................................ 94

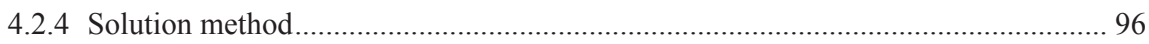

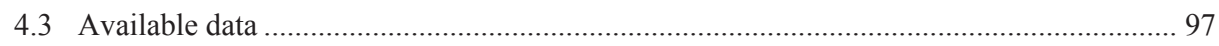

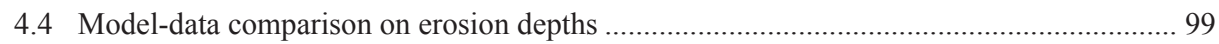

4.4.1 Model-data comparison on grain size dependent erosion behavior........................... 99

4.4.2 Grain-turbulence interaction (I): alternative formulations for fluctuation coefficient $\alpha 99$

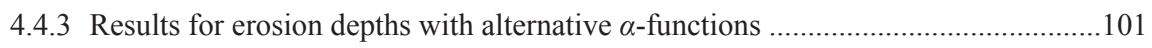

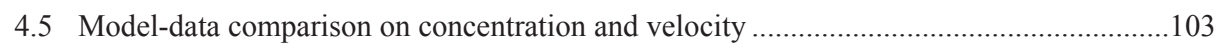

4.5.1 Time-dependent concentration profiles for medium and fine sized sand ..................103

4.5.2 Time-dependent and wave-averaged velocity profiles ............................................105 


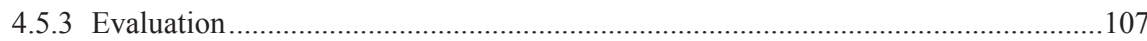

4.5.4 Grain-turbulence interaction (II): further sensitivity tests .......................................108

4.6 Sediment fluxes for fine and medium sized sand in tunnel and flume ..............................109

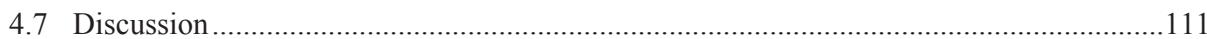

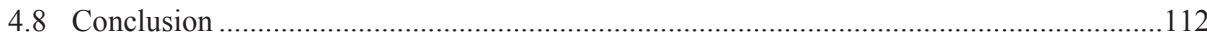

5 Discussion $\quad 115$

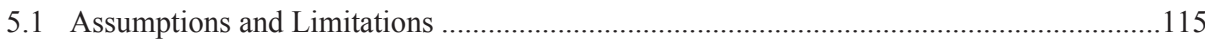

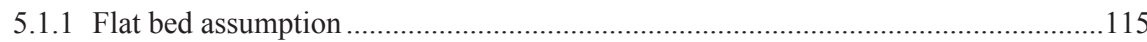

5.1.2 Horizontal bed assumption \& steady, uniform wave assumption ............................116

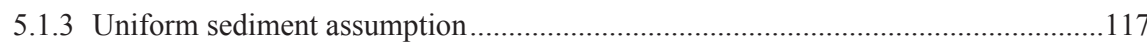

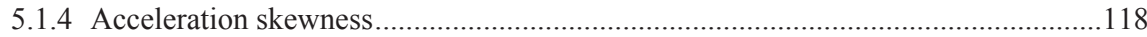

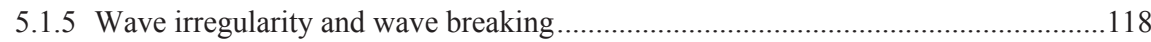

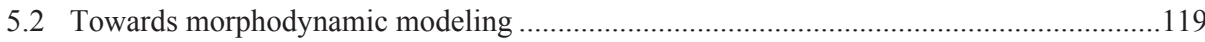

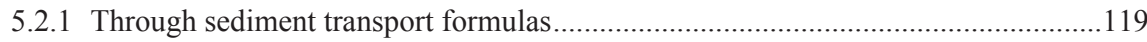

5.2.2 Through direct application of the process-based models........................................119

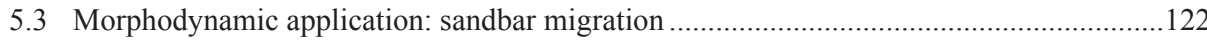

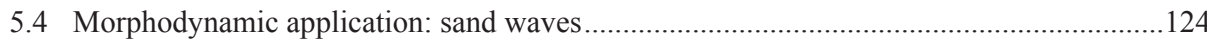

6 Conclusions and recommendations $\quad 127$

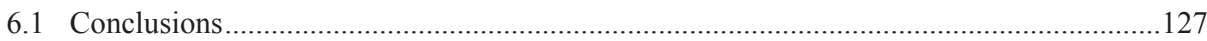

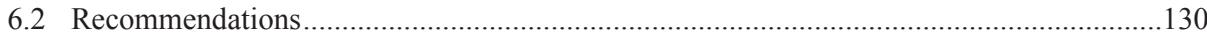

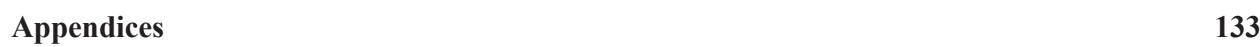

Appendix A: Boundary layer velocities (analytical solutions) ..................................................133

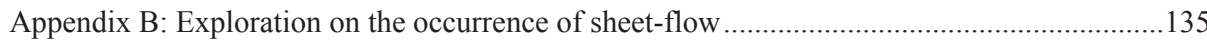

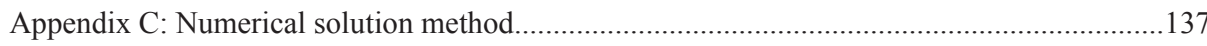

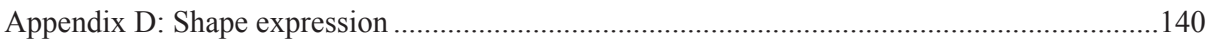

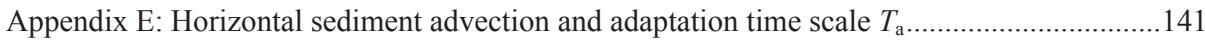

Appendix F: Drag-related terms in momentum and energy equations ......................................144

Appendix G: Particle motion and Stokes number range _...............................................................147

$\begin{array}{ll}\text { References } & 151\end{array}$

$\begin{array}{ll}\text { List of figures } & 161\end{array}$

List of symbols $\quad 163$

Dankwoord / Acknowledgements $\quad 169$

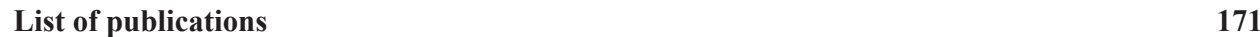

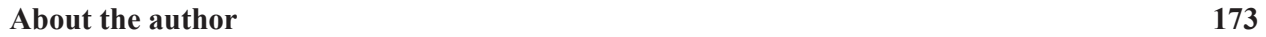




\section{SUMMARY}

In the near-shore zone, water depths are relatively small and wave-related orbital water motions extend down to the sea bed. These motions exert a mobilizing force on the bed sediments. Under relatively energetic near-bed flow, sheet-flow occurs: sedimentary bed forms are washed away and the bed is turned into a dense layer of moving sediment. Sheet-flow has been investigated extensively under laboratory conditions in oscillatory flow tunnels (see Figure 1), considering e.g. the effect of the wave shape or the grain size on sediment transport rates. This research has resulted in semi-empirical formulas for the sediment transport rate, often applied within morphodynamic modeling systems. However, recent sheet-flow experiments in large scale wave flumes (Figure 1) show sediment transport rates rather different from the earlier findings in tunnels. For fine sand under Stokes waves, this even includes a reversal from offshore (tunnels) to onshore (flumes) directed transport. A potential explanation of these observations is 'progressive wave streaming', an onshore directed current present under progressive surface waves, but absent in oscillatory flow. In this thesis we study this streaming and other hydrodynamic differences between tunnels and flumes. We determine how these hydrodynamic differences affect sediment transport, and develop parameterizations to include the additional sediment transporting processes in transport formulas.

Firstly, we focus on the hydrodynamics (chapter 2). We investigate the importance of progressive wave streaming for turbulent boundary layer flow over a fixed rough bed, relative to other current generating processes, especially wave shape streaming. Hereto, we present a numerical 1DV Reynolds-averaged boundary layer model including progressive wave effects. The newly developed model shows good agreement with detailed experimental data on different types of wave boundary layer flow. Next, we determine the balance between progressive wave streaming and wave shape streaming for changing wave and bed conditions from model simulations throughout the parameter domain. This balance, governed by the relative water depth and the relative bed roughness, is subsequently described in parameterizations for the period-averaged boundary layer current and the period-averaged bed shear stress. Thus, our hydrodynamic study results in parameterizations which can be used in transport formulas and a validated numerical tool for the next step of this study.

Secondly, we investigate how hydrodynamic flume - tunnel differences influence sediment transport (chapter 3). Hereto, we use the model of chapter 2, now extended with pick-up, advection and diffusion, and turbulence damping effects of suspended sediment. We demonstrate the good predictive skills of the model in a validation against flow and transport measurements from, amongst others, the recent flume experiments. Next, we quantify the separate contribution of progressive wave streaming and of other flume - tunnel differences to sand transport from numerical model simulations. The results show that progressive wave streaming indeed contributes largely to increased onshore sediment transport rates in flumes. However, especially for fine sand, also the convergence and divergence in horizontal sediment advection in the non-uniform flow field are found to contribute significantly to transport under progressive waves. We therefore conclude that in addition to streaming, also these advection effects should be accounted for in sediment transport 
formulas and morphodynamic models for the near-shore. Hence we present a parameterization of this effect, founded on the numerical model results and an analytical derivation.

Thirdly, we adopt a two-phase continuum model to take a closer look to progressive wave effects on the erosion depth, sheet-flow layer thickness and sediment fluxes inside the sheet-flow layer (chapter 4). We improve the grain size dependent erosion behavior of the model by implementing an alternative formulation for the effects of fluid-grain drag forces on fluid turbulence. This results in good reproductions of measured erosion depths of fine, medium and coarse sized sand beds. Also intra wave concentration and velocity profiles are generally reproduced well, except for some remaining inaccuracies in the fine sand simulations around flow reversal. Next, we apply the model for various grain sizes to predict flux profiles both in oscillatory flow and under progressive waves. From mutual comparison we learn that for fine sand the increased period-averaged flux under influence of progressive waves originates both from the current-related and the wave-related transport contribution. Our exploration shows that this two-phase model can become a valuable instrument for further study and parameterization of sheet-flow layer processes.

The results of this study can be used (some have been used already) in morphodynamic modeling through implementation of the provided parameterizations in sand transport formulas. Alternatively, this study's process-based numerical models can also be applied directly within morphodynamic modeling systems. This is illustrated with a simplified morphological computation concerning sandbar migration. In the example, the predicted sandbar migration speed with and without progressive wave effects differs a factor 2. This clearly emphasizes the need to account for progressive wave effects in morphodynamic models.

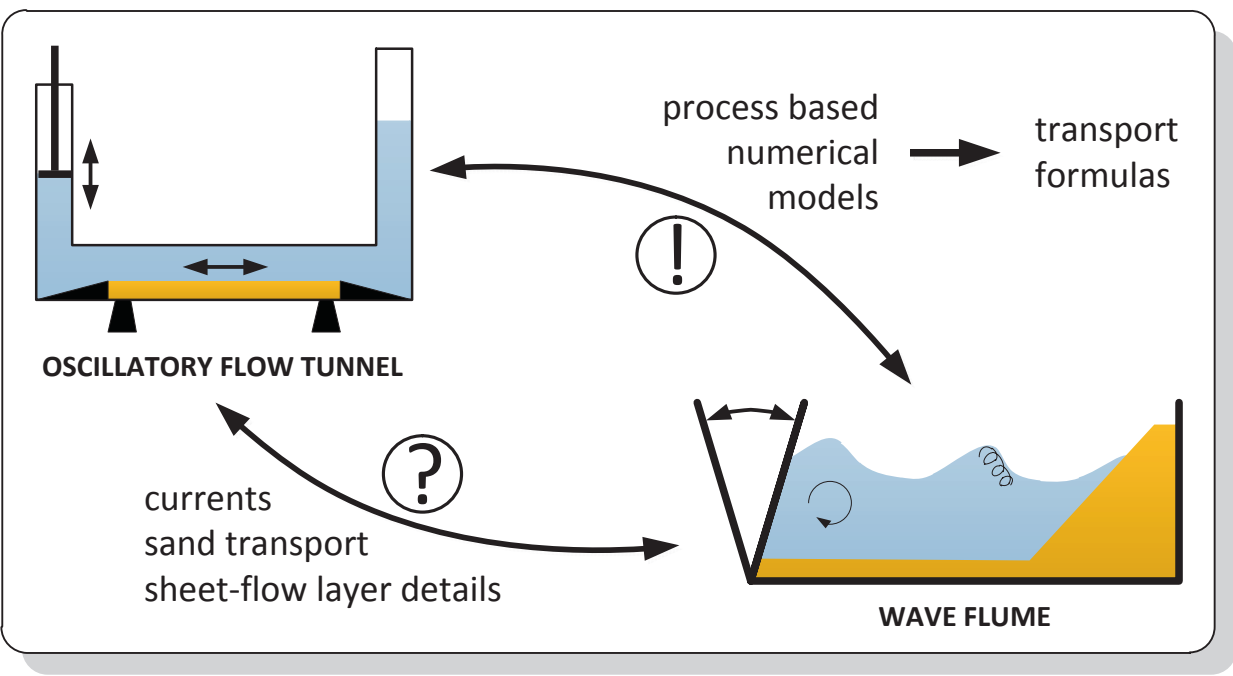

Figure 1: Graphical summary 


\section{SAMENVATTING}

Dichtbij de kust, waar het water relatief ondiep is, is de golf gerelateerde beweging van het water voelbaar tot op de zeebodem. Deze waterbeweging oefent een mobiliserende kracht uit op het bed sediment. In geval van sterke waterbeweging nabij de bodem treedt er sheet-flow op: bodemvormen worden weggespoeld en de bodem verandert in een dichte laag van bewegend sediment. Het fenomeen sheet-flow is uitgebreid onderzocht in laboratorium omstandigheden in zogenoemde oscillatory flow tunnels (zie figuur 1). Hierbij is b.v. gekeken naar de effecten van de golfvorm en de korrelgrootte op de hoeveelheid zand transport per seconde. Dit onderzoek heeft geresulteerd in semi-empirische transport formules, die vaak worden toegepast in morfologische modellen. Echter, recente sheet-flow experimenten in golfgoten (figuur 1) laten sediment transportsnelheden zien die nogal verschillen van de eerdere bevindingen in tunnels. Voor fijn zand onder Stokes' golven houdt dit zelfs een omkering in van de transportrichting: waar tunnel-experimenten zand transport lieten zien van de kust af, laten de golfgoot-experimenten transport zien naar de kust toe. Een mogelijke verklaring hiervoor is progressive wave streaming, een kustwaartse stroming die wel aanwezig is in de prototype situatie en in golfgoten, maar niet in tunnels. Dit proefschrift onderzoekt deze streaming en andere hydrodynamische verschillen tussen tunnels en goten. We bepalen hoe deze hydrodynamische verschillen het sediment transport beïnvloeden en ontwikkelen parametrisaties om de extra sediment transport processen mee te nemen in sediment transport formules.

Hoofdstuk 2 zoomt in op de hydrodynamica: hoe belangrijk is progressive wave streaming voor de totale stroming in een turbulente grenslaag boven vaste, ruwe bodems, in vergelijking met andere stroming genererende processen? Om dit te onderzoeken hebben we een numeriek model ontwikkeld voor de stroming in de bodemgrenslaag waarin de effecten van lopende golven worden meegenomen. Modelsimulaties voor verschillende typen golfgrenslaagstroming laten resultaten zien die goed overeenkomen met gedetailleerde experimentele data. Vervolgens hebben we het model gebruikt om te onderzoeken hoe de invloed van progressive wave streaming verandert ten opzichte van andere processen als de golf- en bodemcondities veranderen. De resultaten hiervan zijn beschreven in parametrisaties voor de golfgemiddelde stroming en bodemschuifspanning, waarin de relatieve waterdiepte en de relatieve bodemruwheid de belangrijkste parameters zijn. Naast deze parametrisaties, die op zich al kunnen worden gebruikt in de ontwikkeling van zand transport formules, is het voornaamste resultaat van dit hoofdstuk het model zelf, want hiermee hebben we een instrument in handen voor de volgende stap.

Hoofdstuk 3 onderzoekt de vraag hoe de hydrodynamische verschillen tussen golfgoten en tunnels uiteindelijk het sediment transport beïnvloeden. Hiertoe gebruiken we het model van hoofdstuk 2 , uitgebreid met modelformuleringen voor het oppikken en transporteren van zand en voor de invloed van gesuspendeerd zand op turbulentie. Eerst valideren we dit model met metingen van zowel stroming als zand transport, onder andere uit de recente golfgoot experimenten. Vervolgens kwantificeren we m.b.v. numerieke simulaties de afzonderlijke bijdrage van progressive wave streaming en van andere verschillen tussen tunnels en golfgoten. De resultaten laten zien dat 
progressive wave streaming inderdaad een aanzienlijk bijdrage levert aan het extra kustwaartse zanstransport in golfgoten. Maar vooral voor fijn zand blijkt ook de afwisselend convergerende en divergerende horizontale advectie van het zand in suspensie aanzienlijk bij te dragen aan het zand transport onder lopende golven. Onze conclusie is daarom dat niet alleen het effect van progressive wave streaming, maar ook het bovengenoemde advectie-effect moet worden meegenomen in formules voor sediment transport en in morfologische modellen. Met het oog hierop sluit hoofdstuk 3 af met een parametrisatie van dit advectie-effect, gebaseerd op een analytische afleiding en resultaten van het numerieke model.

In hoofdstuk 4 gaan we over tot het gebruik van een twee-fase model, met aparte bewegingsvergelijkingen voor water en sediment, om in meer detail te kijken naar het effect van lopende golven op de erosiediepte, de sheet-flow laag dikte en de sediment fluxen binnenin de sheetflow laag. We verbeteren de wijze waarop de modelresultaten voor erosie afhangen van de korrelgrootte door een alternatieve modelformulering te implementeren voor de effecten van gesuspendeerde zandkorreltjes op de turbulentie. Hiermee is het gelukt om voor een range van korrelgroottes de gemeten erosiedieptes te reproduceren. Ook de snelheids- en concentratieprofielen worden over het algemeen goed gereproduceerd, al blijven er in de simulaties met fijn zand enige onnauwkeurigheden aanwezig rondom de omkering van de waterbeweging. Vervolgens simuleren we voor diverse korrelgroottes profielen van de sediment flux in zowel oscillerende stroming als onder lopende golven. Uit de onderlinge vergelijking van de resultaten leren we dat voor het fijne zand de extra sediment flux onder lopende golven zowel een stromings-gerelateerde als een golfgerelateerde component heeft. Dit is in lijn met de resultaten van hoofdstuk 3 en bevestigt onze aanpak van afzonderlijke parametrisatie van de extra transportprocessen on der lopende golven. Verder laat dit hoofdstuk zien dat het twee-fase model in potentie aan waardevol instrument is voor verdere studie en parametrisatie van de processen in de sheet-flow laag.

De resultaten van dit promotieonderzoek kunnen worden toegepast in morphologische modellen door implementatie van de geboden parametrisaties in zand transport formules. Deze ontwikkeling is momenteel ook aan de gang. Daarnaast kunnen de proces-gebaseerde numerieke modellen uit deze studie ook direct worden toegepast binnen een morfologische model. In hoofdstuk 5 wordt deze laatste toepassing geïllustreerd met een eenvoudige morfologische berekening voor de verplaatsing van een zandbank. In het voorbeeld is het verschil in migratiesnelheid met of zonder het loepndegolf-effect een factor 2. Dit onderstreept nog eens de noodzaak om deze effecten mee te nemen in morfologische voorspellingen. 


\section{INTRODUCTION}

\subsection{GENERAL}

Coastal zones are the scene of a wide range of economic and social activities and form valuable and vulnerable environmental systems. To support their various functions, a good understanding and management of coastal systems is essential. A key element herein is the prediction of morphological changes in these systems under influence of natural developments or human intervention. Morphological developments arise from transport of sediments, driven by the water flow originating from e.g. tides, wind, waves, river discharges or density current.

This thesis focusses on wave-related sediment transport processes: we investigate the dynamics of water and sediment in the bottom boundary layer beneath non-breaking waves through numerical modeling. As introduction, section 1.2 gives a brief description of definitions and physical processes most relevant for the motion of water and sediment beneath waves. Subsequently, section 1.3 shortly discusses experimental research, empirical formulas and computational models on wave-induced sediment transport and describes how recent experiments give cause for the present computational modeling study. The research questions central to this thesis are listed in section 1.4, together with the thesis outline.

\subsection{WAVE-INDUCED BOUNDARY LAYERS AND SEDIMENT TRANSPORT}

\subsubsection{Hydrodynamic characteristics}

The wave bottom boundary layer is the near-bed shear layer in which the water motion is not only governed by pressure gradients from the surface waves, but also influenced by friction at the bed.

Propagating surface waves generate orbital water motions: the wave top coincides with maximal orbital velocities in direction of wave propagation, the wave front with maximal upward orbital velocities. At deep water, for sinusoidal waves the orbits are practically circular. The velocity amplitudes decrease with distance from the surface and the influence of the waves does not extend to the bed (Figure 1.1, left). When water depths are smaller than around $1 / 2$ the wave length, the waves start to 'feel the bed': the propagation speed will decrease, causing decreasing wave lengths and increasing wave heights (shoaling). Furthermore, the horizontal velocity amplitudes will be larger than the vertical velocity amplitudes (elliptic orbits) and the near-bed horizontal velocities will be non-zero (Figure 1.1, middle). When the water depth decreases further, the horizontal velocity amplitude becomes nearly constant over depth (Figure 1.1, right). During propagation from deep to shallow water, also the shape of the waves is changing. Firstly, the crest height is amplified compared to the wave trough. Subsequently, the waves start to lean forward (steep front) until they eventually break. 


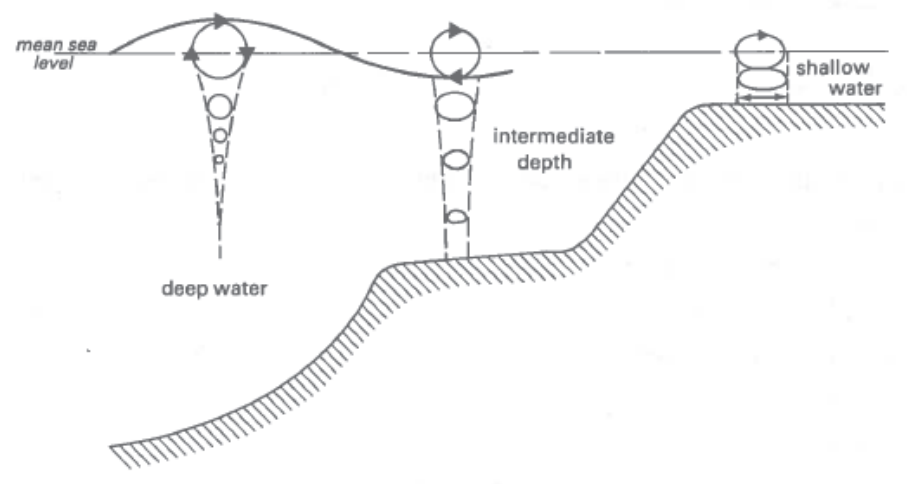

Figure 1.1: Orbital motions under waves for various water depths [figure: Van Rijn, 1990]

In intermediate and shallow water depths, friction will occur between the wave-generated near-bed horizontal velocities and the sea bed. This will introduce shear forces in a thin layer above the bed: the wave boundary layer. In laminar flow, the shear is exerted by viscous stresses. The waveinduced flows of our interest, i.e. relevant for sediment transport, are mostly turbulent. In turbulent flow, the momentum transfer predominantly takes place by turbulent eddies. In analogy with laminar flows, the turbulent momentum transfer is often modeled as a viscous stress, with an eddy viscosity much larger than the kinematic viscosity of water (Boussinesq hypothesis).

The flow inside the viscous or turbulent shear layer shows a number of important characteristics. Firstly, the horizontal flow inside the wave boundary layer is ahead of the near-bed free stream velocity, and this 'phase lead' increases towards the bed. Secondly, the gradual reduction of the horizontal velocity amplitude towards the bed is preceded by a 'velocity overshoot': at certain elevation, the amplitude of the horizontal velocity exceeds the maximum free stream velocity. Figure 1.2 shows analytically obtained profiles of the horizontal velocity amplitude (panel a) and phase (panel b) inside a boundary layer beneath a sinusoidal wave (first order solution, constant viscosity, see appendix A). Herein $z$ is the vertical level above the bed, $\delta_{\mathrm{s}}$ is the Stokes length, $\hat{u}(z)$ and $\hat{u}_{\infty}$ are the horizontal velocity amplitude in the boundary layer and free stream respectively, and $\theta(z)$ is the phase difference between boundary layer and free stream flow.

In the absence of friction ('free stream'), the flow is only accelerated horizontally by the pressure gradient, and horizontal velocities are maximum beneath the wave crest (zero gradient). However, friction forces work against the flow direction and cause flow deceleration as soon as they exceed the force from the pressure gradient. This happens already before the passage of the wave crest. The velocity overshoot arises because the difference between the boundary layer and free stream velocity amplitude behaves as a wave being damped while traveling from the bed upwards. 
(a)

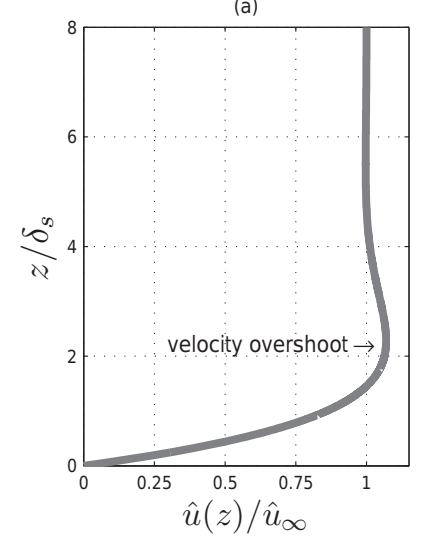

(b)

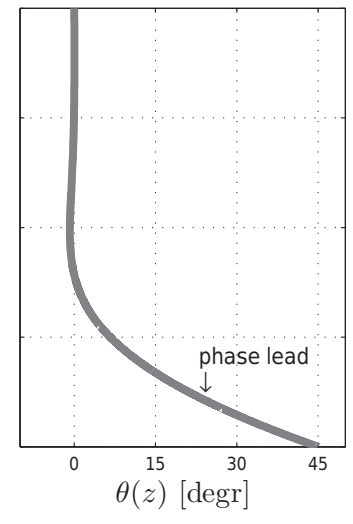

(c)

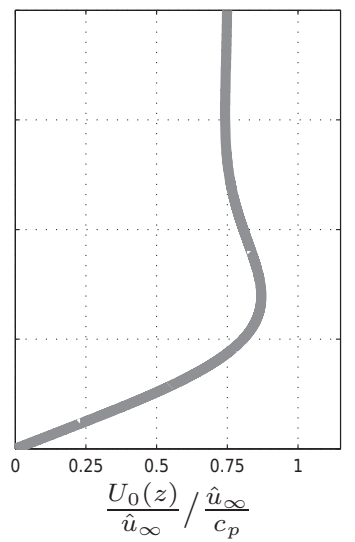

Figure 1.2: Vertical profiles (normalized) of (a) the amplitude and (b) the phase of the horizontal component of the orbital velocity, and (c) the period-averaged current. The shown profiles are analytical solutions for a constant viscosity layer and sinusoidal wave. See appendix A for the mathematical expressions.

A third important characteristic of the boundary layer flow beneath progressive surface waves is the presence of a non-zero wave averaged current ('progressive wave streaming'). The origin of this current can be explained as follows: the vertical velocity at a certain level is the result of the convergence or divergence of the horizontal flow beneath that level (continuity). Because the (depthintegrated) horizontal flow inside the wave boundary layer has a phase lead, also the vertical velocity at the edge of the wave boundary layer will develop a phase lead ( $\Delta \theta$ in Figure 1.3). As a results the horizontal and vertical orbital motion at that level will be more than 90 degrees out of phase. This results in a non-zero wave averaged downward transport of horizontal momentum into the wave boundary layer by the vertical orbital motion. This momentum flux drives a wave-averaged current in the direction of wave propagation ([Longuet-Higgins, 1958]). The generation of this progressive wave streaming is illustrated in Figure 1.3. The analytically obtained current profile (constant viscosity, sinusoidal waves) is shown in Figure 1.2(c). Progressive wave streaming is a key notion in this study.

Other mechanisms that may influence the current inside the boundary layer are the generation of 'wave shape streaming' and of return currents. For waves that have developed a non-sinusoidal form, differences in friction and turbulence appear between the onshore and offshore phase of the wave. For waves with amplified crests, this gives rise to a wave-averaged boundary layer current against the propagation direction. The generation of wave shape streaming, firstly predicted by Trowbridge and Madsen [1984] and firstly observed by Ribberink and Al-Salem [1995], is illustrated in Figure 1.4. Return currents are currents compensating wave-averaged mass and momentum transport in wave propagation direction from e.g. Stokes drift or wave breaking. The transport 


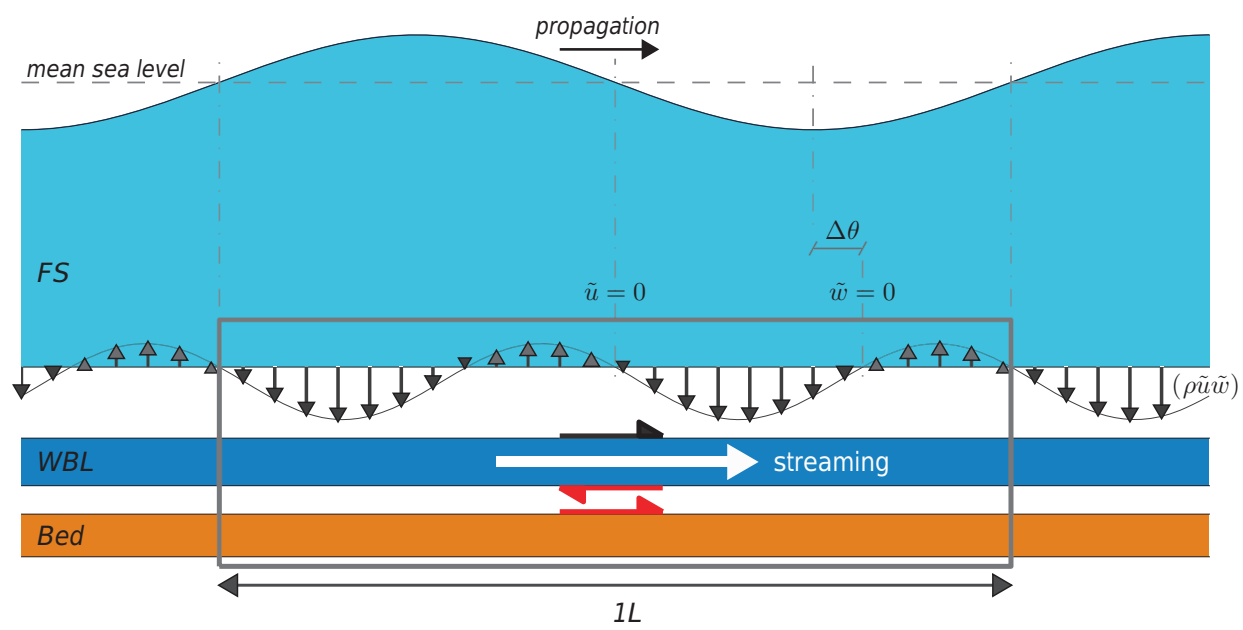

Figure 1.3: Schematic representation of the generation of streaming beneath sinusoidal progressive waves; Averaged over a wave, the exchange of horizontal momentum between the free stream (FS) and the wave boundary layer (WBL) (the vertical arrows, denoting $\rho \tilde{u} \widetilde{w}$ ) results in a net downward momentum transport, i.e. a positive stress on the top of the WBL (black shear arrow). This stress drives a boundary layer current (streaming) in direction of wave propagation till the wave-induced stress is balanced by the current related bed shear stress (red shear arrows). Symbols $\tilde{u}$ and $\tilde{w}$ : horizontal and vertical component of the orbital velocity (at the edge of the boundary layer). $\Delta \theta$ : phase lead of $\tilde{w}$ compared to the situation without friction.

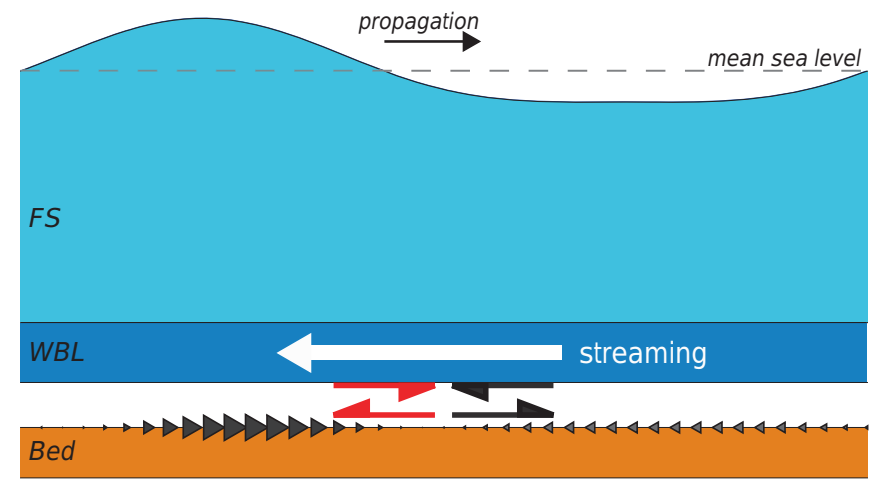

Figure 1.4: Schematic representation of the generation of streaming beneath Stokes waves (amplified crest); Averaged over a wave, wave-related shear stresses on the bed (black and gray triangles) are onshore directed (black shear arrow), equivalent to an offshore directed stress on the WBL (black shear arrow). This stress drives a boundary layer current (streaming) against the direction of wave propagation till the waveinduced stress is balanced by the current related stress (red shear arrows). 
towards the 'closed' coast generates a pressure gradient that subsequently drives an offshore current. Note that the return current generating mass and momentum transport predominantly occurs near the surface and in the upper part of the free stream [Svendsen, 1984]. On the other hand, progressive wave streaming and wave shape streaming are typical wave boundary layer phenomena.

\subsubsection{Sediment transport regimes}

Not only the near-bed flow, but also the bed will be affected by the friction between the flow and the bed. Under influence of the flow, individual sand grains at the bed are mobilized and subsequently transported with the flow. Various regimes of wave-induced sand transport can be distinguished, connected to the ratio of mobilizing forces due to drag and lift and stabilizing forces due to the grain's immersed weight, reflected by the Shields parameter $\theta$ :

$$
\theta=\frac{\tau_{b}}{\left(\rho_{s}-\rho_{w}\right) g D}
$$

were $\tau_{\mathrm{b}}$ is the bed shear stresss, $\rho_{\mathrm{s}}$ the density of sand, $\rho_{\mathrm{w}}$ the density of water, $g$ the gravitational acceleration and $D$ the grain diameter. In order of increasing mobilizing forces, one distinguishes:

No-transport regime: Below a certain threshold of motion (critical Shields parameter), the wavegenerated forces are too small to mobilize the grains.

Ripple regime: Above the threshold of motion, the grains start to move, roll over the bed and form small ridges (rolling-grain ripples). For increasing Shields parameter, vortex ripples will develop: the flow over the ripples generates vortices that erode sand from the ripple troughs and bring it towards the ripple crest. Net sediment transport occurs when these ripples migrate e.g. due to nonsinusoidal wave shapes.

Sheet-flow regime: For increasing Shields parameter, transition to sheet-flow occurs $(\theta>0.8$, [Wilson, 1989]). Characteristics of this phenomenon are that ripples are washed out from the bed, which becomes flat again, and that the motion of sediment extends down to several grain diameters below the initial bed level. The moving layer with high sediment concentrations causes very large sediment transport rates. Sheet-flow sediment transport is regarded as the dominating regime for near-shore morphological changes during energetic wave conditions, and is the focus of the present thesis.

To illustrate the relevance of sheet-flow sediment transport, Table 1.1 and Figure 1.5 present the results of an exploration on the occurrence of sheet-flow in front of the Dutch coast. This brief exploration (see Appendix B) consisted of three steps: (1) analysis of data of a wave buoy in front of the Dutch coast to obtain a schematized wave climate (i.e. functions relating wave period and probability of exceedance to the wave height); (2) construction of representative deep water wave conditions and translation of these conditions into wave heights and near bed velocities in the near shore area; (3) determination of the depth where the sheet-flow criterion is met. Table 1.1 gives the deep water wave height and the wave period for waves with a probability of exceedance of 50, 20, 
10 and $1 \%$ as derived from 35 years of data from wave buoy YM6 (IJmuiden munitiestortplaats). Figure 1.5 gives - for two median sand grain sizes in the range occurring in front of the Dutch coast - the water depth where the sheet-flow criterion is met as function of the deep water wave height. Notwithstanding its strong simplifications, this example indicates that for $d_{50} \approx 0.20 \mathrm{~mm}$ sheet-flow may occur as from the $7 \mathrm{~m}$ water depth contour for about $20 \%$ of the time, and already at the $10 \mathrm{~m}$ contour for about $10 \%$ of the time. Note that with the large sediment transport rates involved, the relative contribution of sheet-flow to the total sediment transport will strongly exceed its percentage of occurrence.

Table 1.1: Schematized deep water wave characteristics in front of the Dutch coast (see appendix B for the derivation).

\begin{tabular}{ccc}
\hline $\begin{array}{c}\text { Probability of } \\
\text { Exceedance (\%) }\end{array}$ & $\begin{array}{c}\text { Deep Water } \\
\text { Wave Height (m) }\end{array}$ & Wave Period (s) \\
\hline 50 & 1.1 & 5.4 \\
20 & 1.9 & 6.1 \\
10 & 2.5 & 6.6 \\
1 & 4.4 & 8.3 \\
\hline
\end{tabular}

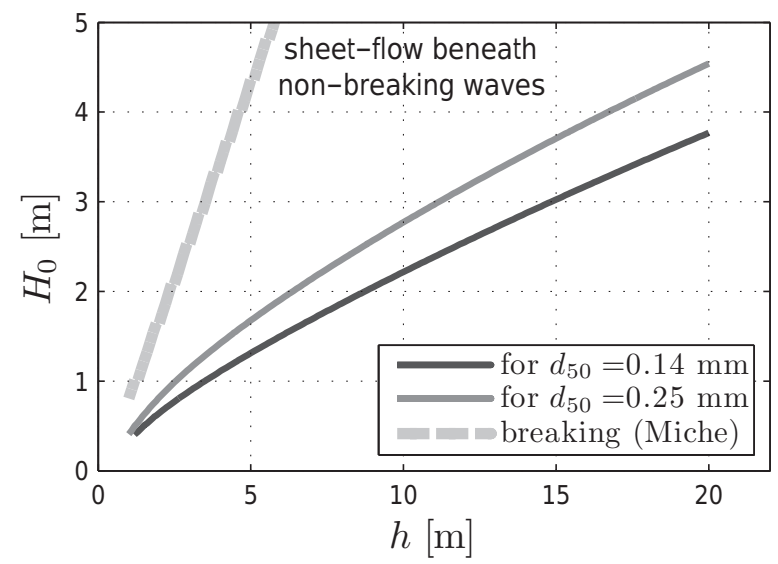

Figure 1.5: Parameter space delineation for sheet-flow beneath non-breaking waves. Lines: water depth $h$ where the sheet-flow criterion is met as function of deep water wave height $H_{0}$. Left of the lines, sheet-flow may be expected. Dashed line: breaking limit according to Miche $(H / h=0.88)$. 


\subsection{RESEARCH CONTEXT}

This section provides a brief discussion of experimental research, empirical formulas and computational models on wave-induced sediment transport. It furthermore describes how recent experiments give reason for the computational modeling study presented in this thesis.

\subsubsection{Laboratory facilities}

Field measurements on wave-induced boundary layer flow and sediment transport are difficult to obtain, especially under the energetic wave conditions generating sheet-flow. Most research on wave boundary layer processes is therefore carried out in laboratory facilities. These facilities enable researchers to gather detailed measurements of flow, sediment concentration and transport and to investigate varying wave and bed conditions systematically in well-controlled circumstances. Basically, two types of laboratory facilities are used: Oscillating Flow Tunnels and Wave Flumes (Figure 1.6).

In Oscillating Flow Tunnels the wave-induced near-bed water motion in intermediate and shallow water is simulated by a horizontally uniform oscillating flow. This flow is generated in a U-tube, with a horizontal test section with rigid lid in the middle and reservoirs at either end. The oscillatory water motion results from a moving piston at one end and pressure from water accumulation in the opposite open reservoir. The special advantage of such tunnel facilities is the possibility to mimic near-bed flow with prototype flow velocities and oscillation periods in relatively small facilities. This way, all difficulties and uncertainties related to scaling of turbulence and sediment related processes are eliminated and the empirical insights can be directly applied in engineering problems. In Oscillating Flow Tunnels the vertical component of the orbital velocity is absent and related wave-induced currents are not reproduced.

Wave Flumes are longitudinal reservoirs, at one end equipped with a wave generator to produce propagating surface waves. In such facilities, entire cross shore profiles can be physically modeled and cross shore wave propagation, flow phenomena, sediment transport and profile development can be investigated. Wave Flumes allow for a more complete representation of the processes in the field. However, experiments at prototype scale need large facilities and are costly, while experiments at smaller scale introduce scaling problems. Only few full scale wave flume experiments on sheet-flow sediment transport have been reported in literature and the investigated wave and bed conditions are limited in range and less well-controlled. 


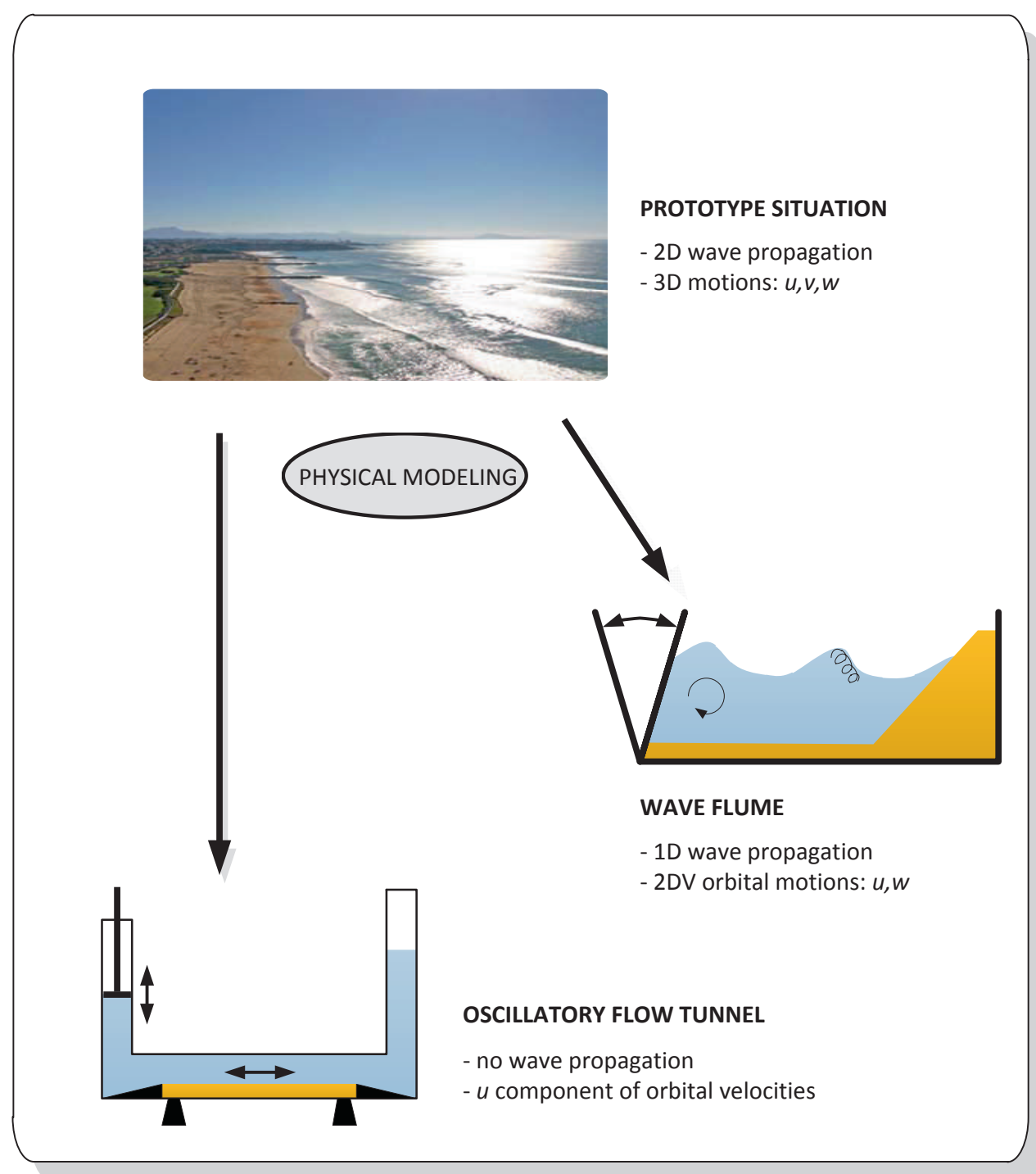

Figure 1.6: Laboratory facilities for research on wave-induced sediment transport and there most important characteristics compared to prototype situation. 


\subsubsection{Empirical formulas}

Laboratory experiments over the last fifty years on wave (or oscillating) boundary layer flow over both fixed and mobile beds have provided numerous insights in the dynamics of water and sand under waves. Over time, these insights have become available for engineering practice trough empirical formulas for e.g. boundary layer thickness, wave-induced friction, sheet-flow layer thickness, and through practical sediment transport formulas.

A key insight concerning boundary layer flow is that in the turbulent flow regime the structure of the boundary layer depends on the roughness of the bed relative to the orbital excursion. Based hereon, various authors have proposed formulas for the boundary layer thickness and friction factor, e.g. Jonsson [1966], Swart [1974], Kamphuis [1975], Jonsson [1980], Sleath [1987], Fredsøe and Deigaard [1992] and Nielsen [1992]. Measurements on behavior of the sheet-flow layer under waves have been summarized in expressions for the sheet-flow layer thickness by e.g. Wilson [1989], Sumer et al. [1996] and Ribberink et al. [2008].

Sediment transport formulas are semi-empirical formulations that relate the wave-induced, timedependent transport to the (free stream) horizontal flow velocity or bed shear stress. A distinction can be made between 'quasi-steady' and 'semi-unsteady' transport formulas. Quasi-steady formulas directly relate the instantaneous transport to the instantaneous velocity or stress through power laws and empirical coefficients (e.g. Madsen and Grant [1976], Bailard [1981], Trowbridge and Young [1989], Ribberink [1998], Nielsen [2006], Van Rijn [2007]). Transport formulas are mainly based on tunnel experiments, and over time much effort has been spent to incorporate newly investigated conditions and processes, e.g. wave shape influence (investigated by Ribberink and Al-Salem [1995] and Van der A et al. [2010]), grain size effects (Dibajnia and Watanabe [1992], Dohmen-Janssen et al. [2002], O'Donoghue and Wright [2004]), size gradation effects [Hassan and Ribberink, 2005] and sediment transport in the ripple regime [Van der Werf et al., 2007]. An important insight, especially from the studies on grain size and ripple effects, is that sediment concentration and sediment transport do not always react instantaneously to changes in the flow velocity. In case of ripples and fine sand sheet-flow, concentration and transport show a phase lag with respect to the free stream velocity. Semi-unsteady transport formulas are formulas that account for the effects of these phase lags on the net transport rate. Examples are Dibajnia and Watanabe [1998], DohmenJanssen et al. [2002], and Van der A et al. [2011]. Sand transport formulas fulfill an important role in morphodynamic modeling, because they provide the possibility to predict the wave-induced net sediment transport without simulations of flow and transport on (intra) wave period and (intra) boundary layer time and length scale.

\subsubsection{Process-based intra wave boundary layer models}

Next to experiments, also process-based modeling is applied to investigate the flow and sand transport mechanisms in the wave boundary layer (WBL). Parallel to the physical modeling studies, also the computational modeling studies mostly consider horizontally uniform oscillating flows. Contrary to the semi-empirical formulas, process-based intra WBL models explicitly compute the 
(turbulence averaged) time-dependent flow inside the WBL. Among the turbulence averaged intra WBL models, we can distinguish (I) (quasi-)single phase models and (II) two phase models.

Models of the first type solve the (horizontal) flow velocities from Reynolds averaged Navier-Stokes (RANS) equations, while sediment concentrations are solved from an advection-diffusion equation. This assumes that, apart from sediment settling, the sand moves with the fluid velocity. Examples of this type of model are e.g. Fredsøe et al. [1985], Hagatun and Eidsvik [1986], Davies and Li [1997], Holmedal et al. [2003], Henderson et al. [2004]. Differences between these models appear in the adopted turbulence closure (e.g. $k-\varepsilon, k-\omega, k-L$ turbulence model) and in the extent to which the model accounts for effects of sediment concentration on water and sediment motions. Single phase models have been helpful tools to investigate the effect of the wave shape [Holmedal and Myrhang, 2006], [Ruessink et al., 2009], sediment-induced stratification [Conley et al., 2008], grain size variations [Hassan and Ribberink, 2010], and combined wave and currents [Li and Davies, 1996], [Holmedal et al., 2004] on wave-induced sediment transport.

In two phase continuum models, the fluid and sediment motions are computed from separate turbulence averaged mass and momentum equations for both the fluid and sediment phase, coupled through fluid-sediment interaction forces. In principle, a more accurate description of the sand motion within the highly concentrated sheet-flow layer is made possible with two phase models, because these models explicitly account for the various forces driving the sediment motion. However, hereto proper descriptions of the various interaction forces are needed. Furthermore, as consequence of including a second set of flow equations for the sediment phase, also a closure is needed for the 'turbulent' inter-granular stresses. Examples of two phase continuum models are Asano [1990], Dong and Zhang [1999], Hsu et al. [2004], Teakle [2006], Amoudry et al. [2008], Li et al. [2008]. Again, the main differences between the various models appear in the closures. For the fluid stresses, both mixing length, one and two equation turbulence models are applied. Intergranular stresses are modeled with either rheological equations (e.g. [Bagnold, 1954], [Ahilan and Sleath, 1987]) or a 'granular temperature' for the energy of the turbulent particle fluctuations [Jenkins and Hanes, 1998]. At present, two phase models start to become helpful tools for parameterization of 'micro processes' like bed erosion [Chen et al., 2011] and sediment pick-up [Yu et al., 2012].

\subsubsection{Motive for the present study}

The motive for the present study lies in observations made during large scale wave flume experiments on sheet-flow sediment transport. Dohmen-Janssen and Hanes [2002] measured significantly more onshore sediment transport than reported earlier for tunnel experiment with comparable sediment and comparable horizontal velocities in the free stream. More recently, Schretlen [2012] found even a reversed transport direction for fine sand in the wave flume (onshore) compared to tunnel experiments (offshore). Therefore, the question is whether the differences in transport can be explained from the hydrodynamic differences between the experimental facilities, and how processes not considered in tunnel experiments can be accounted for in practical sediment 
transport formulas. Dohmen-Janssen and Hanes [2002] formulated the hypothesis that effects of the small onshore directed progressive wave streaming - being absent in oscillating flow tunnels - on flow and sheet-flow sand transport processes are the major explanation for the found differences in transport rates. These questions and hypothesis are the starting-point of the present study. We will investigate this using process-based intra wave boundary layer models. In complement to physical experiments, these models allow us to investigate a wider range of wave and bed conditions and to isolate processes and their effects on transport for parameterization in aid of sediment transport formulas. development.

\subsubsection{PSM model}

Next to experimental studies, also numerical studies exist that point at the large potential influence of progressive wave induced streaming on sediment transport. Bosboom and Klopman [2000] predicted increased onshore transport under propagating free surface waves compared to horizontally uniform oscillating flow on the basis of numerical experiments with the 1DV Point Sand Model (PSM) ([Uittenbogaard, 2000], [Uittenbogaard et al., 2001]). This model can be classified as a non-hydrostatic single phase RANS model. It solves the fluid velocity and sediment concentration throughout the water column, including the WBL. In the PSM model, a spectral / harmonic approach is adopted: the various harmonic components of the vertical and horizontal velocity are solved consecutively from harmonic components of the water level elevation through linearized Poisson equations. The wave component related contribution to the period-averaged current is subsequently determined through exchange of period-averaged momentum between intrawave and wave averaged motions. Within this project, we started our study on progressive wave streaming and its influence on sediment transport with the original PSM model. Although we have found good reproductions of measured wave-generated current profiles for linear waves, we did not manage to achieve steady and accurate results for the current under non-linear waves (with multiple harmonic components). Considering that non-linear wave shapes are of utmost importance for sediment transport and that sediment transport mostly takes place inside the wave boundary layer, we have left the spectral approach during this project and report here only on our activities to implement/investigate free surface effects into/with hydrostatic, wave boundary layer models. The latter approach allows for computation of the combined mean and orbital horizontal velocity without numerical procedures to exchange momentum between various components of the motion.

\subsection{THESIS AIM, RESEARCH QUESTIONS AND OUTLINE}

\subsubsection{Main objective}

The main objective of this study is to develop a detailed understanding of the effects of progressive wave streaming on boundary layer flow and sheet-flow sand transport processes beneath surface waves for realistic wave and bed conditions by development, validation and application of numerical models for wave-induced sediment transport. 


\subsubsection{Approach}

A good understanding of the hydrodynamics is a pre-requisite for understanding sand transport mechanisms. For that reason, the methodology of the present study is to focus first on the wave boundary layer flow over fixed beds. Subsequently, the effect of progressive wave streaming on sediment transport rates is investigated without considering all the details of the processes within the sheet-flow layer. Finally, typical sheet-flow layer processes related to the strong erosion of the bed in the sheet-flow regime are investigated in more detail.

The method adopted in this study is process-based numerical modeling. Within each project step described above, we extend an existing model with formulations essential to investigate the effects of progressive wave streaming for either flow, transport or detailed sheet-flow layer processes under various wave and bed conditions. In each step, the model development is validated with data especially relevant for that specific step. Subsequently, the model is applied to investigate the relative importance of progressive wave streaming compared to other processes by numerically isolating separate processes and exploring the parameter domain. Next, parameterizations are developed to implement the newly obtained insights in practical sediment transport formulations for morphodynamic modeling.

\subsubsection{Research questions and outline}

The research objective and approach are further specified by the following research questions and thesis outline (see Figure 1.7).

RQ1: How can we develop process-based numerical tools to investigate the effects of progressive wave streaming on flow, transport and detailed sheet-flow layer processes for realistic wave and bed conditions?

Elementary, progressive wave streaming is connected to the vertical advection of horizontal momentum. Whether process-based models account for streaming, depends directly on the question whether this advection process is present in the model formulation. However, to investigate its effect on flow, transport and sheet-flow layer details for realistic wave and bed conditions, also other model features are relevant. The features are discussed for flow, transport and sheet-flow layer details in the sections 2 of respectively chapter 2,3 and 4 .

RQ2: How important is progressive wave streaming for the turbulent boundary layer flow above a fixed rough bed relative to other current generating processes, especially wave shape streaming? How do changes in wave and bed conditions affect the balance between these processes?

This question is discussed in chapter 2. After describing the developed numerical Reynolds-averaged hydrodynamic boundary layer model with free surface effects, this chapter describes the model validation using selected laboratory measurements of different types of wave boundary layer flow (fixed beds). The successful validation allows us to answer the question from model simulations for 
various wave and bed conditions, reflected by the relative water depth $k h$ and relative bed roughness $A / k_{\mathrm{N}}$. Chapter 2 also gives a parameterization of the results for streaming velocities and additional wave-averaged bed shear stresses to include streaming in practical sand transport formulas for morphodynamic modeling.

RQ3: To what extent is progressive wave streaming important for sheet-flow transport of fine and medium sized sand, relative to other transport generating effects of the free surface wave? How do changes in wave and bed conditions affect the role of these processes?

This question is investigated in chapter 3 with the hydrodynamic model of chapter 2 extended with formulations describing the pick-up, the advective and diffusive transport and the turbulence damping effects of suspended sediment. The model validation includes a comparison with the recently obtained full scale flume measurements of Schretlen [2012] on both flow and transport. The importance of progressive wave streaming and other free surface effects is quantified from numerical simulations for various wave and bed conditions and the results are parameterized.

RQ4: What is the influence of progressive wave streaming and other free surface effects on the erosion depth, sheet-flow layer thickness and the sediment flux taking place within the sheet-flow layer? How do these effects differ for various realistic grain sizes?

This question, discussed in chapter 4, is investigated using a two-phase model that describes the processes inside the sheet-flow layer in more detail. However, to investigate erosion depth and fluxes for both medium and fine sized sands, a further development turned out to be needed concerning the model's turbulence closure. Chapter 4 describes the model development and the validation using detailed flow and concentration measurements inside the sheet-flow layer. Subsequently, trends in sediment flux profiles under influence of grain size variation and free surface effects are investigated from numerical simulations.

Chapter 5 and 6 form the closure of this thesis. Chapter 5 discusses the main assumptions behind the process-based models and the potential consequences of neglected aspects. Next, it discusses how the results of the present study can be used in morphodynamic modeling and illustrates the potential implications hereof for morphodynamic predictions. Chapter 6 summarizes the answers to the research question and gives recommendations for further research. 


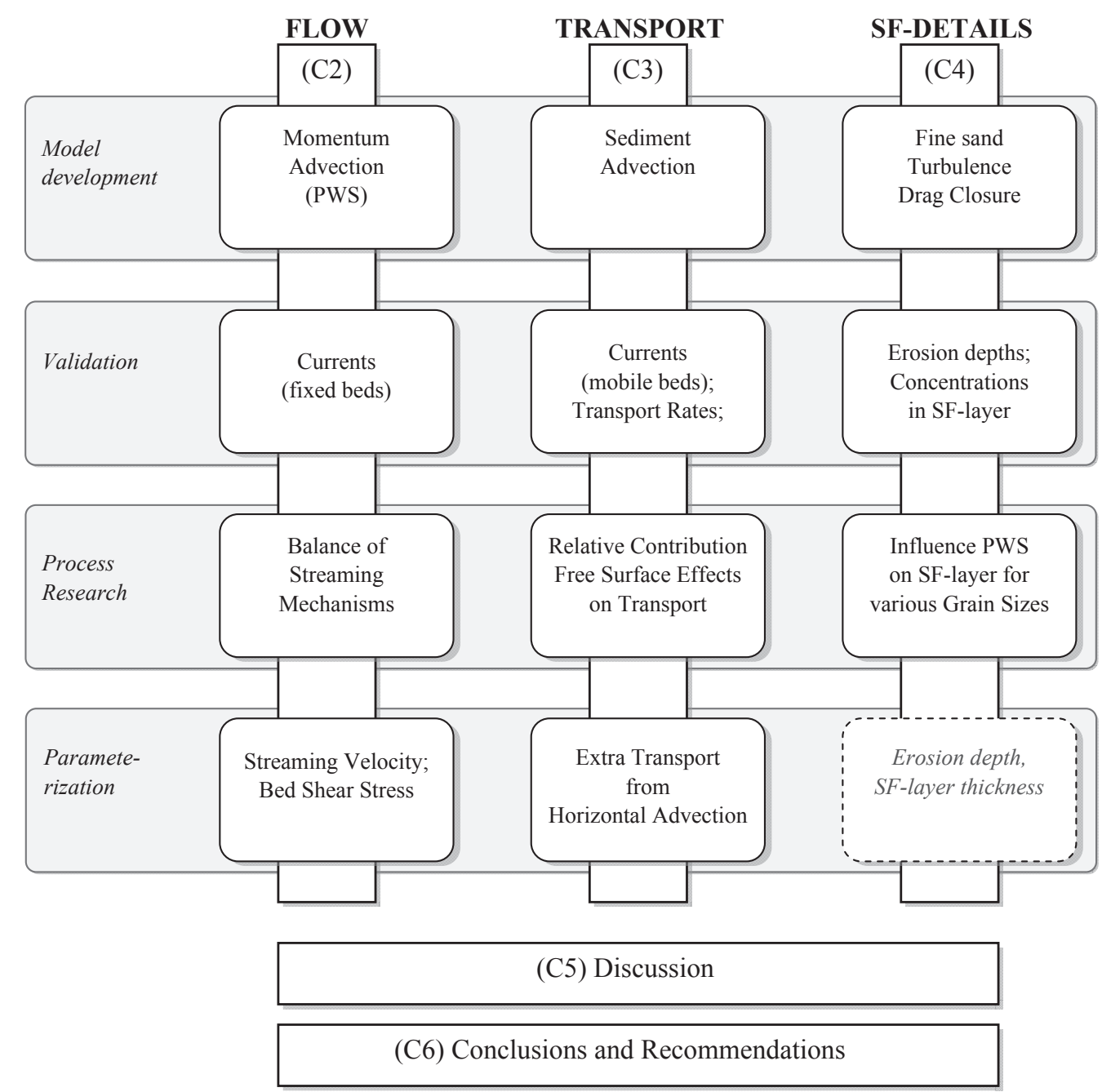

Figure 1.7: Schematic overview of thesis methodology and outline 


\title{
2 NET CURRENTS IN THE WAVE BOTTOM BOUNDARY LAYER: ON WAVE SHAPE STREAMING AND PROGRESSIVE WAVE STREAMING ${ }^{1}$
}

\begin{abstract}
The net current (streaming) in a turbulent bottom boundary layer under waves above a flat bed, identified as potentially relevant for sediment transport, is mainly determined by two competing mechanisms: an onshore streaming resulting from the horizontal non-uniformity of the velocity field under progressive free surface waves, and an offshore streaming related to the non-linearity of the wave shape. The latter actually contains two contributions: oscillatory velocities under non-linear waves are characterized in terms of velocity-skewness and acceleration-skewness (with pure velocity-skewness under Stokes waves and acceleration-skewness under steep sawtooth waves), and both separately induce offshore streaming. This paper describes a 1DV Reynolds-averaged boundary layer model with $k-\varepsilon$ turbulence closure that includes all these streaming processes. The model is validated against measured period-averaged and time-dependent velocities, from 4 different welldocumented laboratory experiments with these processes in isolation and in combination. Subsequently, the model is applied in a numerical study on the wave shape and free surface effects on streaming. The results show how the dimensionless parameters $k h$ (relative water depth) and $A / k_{\mathrm{N}}$ (relative bed roughness) influence the (dimensionless) streaming velocity and shear stress and the balance between the mechanisms. For decreasing $k h$, the relative importance of wave shape streaming over progressive wave streaming increases, qualitatively consistent with earlier analytical modeling. Unlike earlier results, simulations for increased roughness (smaller $A / k_{\mathrm{N}}$ ) show a shift of the streaming profile in onshore direction for all $k h$. Finally, the results are parameterized and the possible implications of the streaming processes on sediment transport are shortly discussed.
\end{abstract}

\footnotetext{
${ }^{1}$ This chapter has been published as: Kranenburg, W.M., J.S. Ribberink, R.E. Uittenbogaard and S.J.M.H. Hulscher (2012), Net currents in the wave bottom boundary layer: on wave shape streaming and progressive wave streaming, Journal of Geophysical Research, 117(F03005),

DOI:10.1029/2011JF002070.
} 


\subsection{INTRODUCTION}

The dynamics of water and sediment in the bottom boundary layer under waves in coastal seas are of key importance for the development of cross-shore and long-shore coastal profiles. Many recent studies on the complex interaction between wave motion and sea bed emphasize the influence of the wave shape on bed shear stress, sediment transport and flow velocities, either focusing on velocityskewness (present under waves with amplified crests), acceleration-skewness (present under waves with steep fronts) or both phenomena in joint occurrence (for references see Ruessink et al. [2009]). Experimental studies on wave shape effects have often been carried out in oscillating flow tunnels, with both fixed and mobile beds of various sand grain sizes, and special attention has been paid to the sheet-flow transport regime, where bed forms are washed away and the bed is turned into a moving sediment layer [Ribberink et al., 2008]. An important observation from tunnel experiments in the sheet-flow regime is that under velocity-skewed flow over coarse grains the sediment transport is mainly onshore, but that net transport decreases with decreasing grain sizes and can even become negative for fine sand [O'Donoghue and Wright, 2004]. Dohmen-Janssen and Hanes [2002] and very recently Schretlen et al. [2011] carried out detailed full-scale wave flume experiments on sand transport by waves in the sheet-flow regime. These flume measurements show onshore instead of offshore transport of fine sand under $2^{\text {nd }}$ order Stokes waves and larger transport rates for medium sized sand compared to experiments with comparable velocity-skewness in oscillating flow tunnels. These different results for sediment transport emphasize the importance of a good understanding of the hydrodynamic differences between oscillating flow tunnels, with horizontally uniform oscillating pressure gradients, and wave flumes, with horizontally non-uniform pressure gradients and vertical motions due to the free surface.

A remarkable free surface effect that potentially contributes to onshore (current related) sediment transport is the generation of a steady bottom boundary layer current in onshore direction [LonguetHiggins, 1953]: the vicinity of the bed affects the phase of the horizontal and vertical orbital velocities. This introduces a wave-averaged downward transport of horizontal momentum that drives an onshore boundary layer current (here called 'progressive wave streaming'). This process acts opposite to the net current that will be generated in a turbulent bottom boundary layer by a velocityskewed or acceleration-skewed oscillation ('wave shape streaming'). The latter mechanism, that can be present both in tunnels and flumes, is due to the different characteristics of the time-dependent turbulence during the on- and offshore phase of the wave, introducing a non-zero wave-averaged turbulent shear stress. This phenomenon was firstly predicted for velocity-skewed waves by Trowbridge and Madsen [1984b] and observed in tunnel experiments by Ribberink and Al-Salem [1995].

It is the aim of this study to develop a carefully validated numerical model for the net currents in the turbulent wave boundary layer above a flat but hydraulically rough bed, and to develop more insights in the balance between the wave shape streaming and progressive wave streaming on the shoreface. 
The various streaming contributions have been modeled before by several authors: Longuet-Higgins [1958] predicted the onshore streaming under progressive waves analytically using a constant viscosity. Johns [1970] included height-dependency in the eddy viscosity and later [Johns, 1977] used a turbulent kinetic energy closure in a numerical study on the residual flow under linear waves. Trowbridge and Madsen [1984a] developed an analytical model with time dependent eddy viscosity. Their second order approach [Trowbridge and Madsen, 1984b] (TM84) jointly included 1) the advective terms of the momentum equation, 2) (forcing) free stream velocities determined with Stokes' 2nd order wave theory, and 3) an eddy viscosity being the product of a vertical length scale and the first three Fourier components of the shear velocity. This key development revealed the competition between onshore progressive wave streaming and offshore velocity-skewness streaming, with dominance of the latter for relatively long waves. Later work [Trowbridge and Young, 1989] and a recent coupling of the TM84 model with a bed load transport formula [Gonzalez Rodriquez, 2009, chapter 6] indeed showed a significant effect of progressive wave streaming on shear stress and net bed load transport. Due to the absence of detailed flume measurements and just tunnel data available for validation, progressive wave streaming was not included in most of the (one and two phase) numerical boundary layer models developed for research on shear stress and sediment transport under waves [e.g. Davies and Li, 1997; Holmedal and Myrhaug, 2006; Conley et al., 2008; Fuhrman et al., 2009a; 2009b; Hassan and Ribberink, 2010; Hsu and Hanes, 2004; Li et al., 2008; Ruessink et al., 2009]. Such models, both with one and two-equation ( $k-\varepsilon$ and $k-\omega)$ turbulence closures, are generally fairly well capable to reproduce the velocity-skewness streaming as measured in tunnels by Ribberink and Al-Salem [1995]. These Reynolds-averaged models have recently been supported by results of Direct Numerical Simulations [Cavallaro et al., 2011], have been used in a 2D version to investigate slope effects in tunnels [Fuhrman et al., 2009a] and have shown good reproduction of measured sediment transport rates in tunnels as well [e.g. Ruessink et al., 2009; Hassan and Ribberink, 2010]. To the author's knowledge, only a few studies ([Henderson et al., 2004], [Hsu et al., 2006], [Holmedal and Myrhaug, 2009] and [Yu et al., 2010]) have presented numerical boundary layer models that include effects of the free surface and the wave shape on the boundary layer flow simultaneously. These studies demonstrate respectively the relevance of progressive wave streaming for onshore sand bar migration (first two references, validation on morphological field data), for streaming profile predictions (third reference, without data-model comparison) and for suspended sediment transport (fourth reference, validation on concentration profiles). Nevertheless, a detailed validation of the numerical models on net current measurements is still lacking until now.

Considering the experimental observations and indications from the model studies, the research objectives in this study are: i) to validate the hydrodynamics of a numerical Reynolds-averaged boundary layer model, extended with free surface effects, using selected laboratory measurements of different types of wave boundary layer flow, ii) to apply this model to obtain insight in the balance between progressive wave streaming and wave shape streaming, and how this is affected by varying wave and bed conditions. Our model, basically an extension of the model used in [Ruessink et al., 2009] and [Hassan and Ribberink, 2010], is described in section 2. The model validation on detailed 
velocity measurements above fixed beds is given in section 3. The balance between progressive wave streaming and velocity-skewness streaming is studied with a systematic numerical investigation of velocities and shear stresses in section 4. Section 5 gives a short outlook on the implications of modeling these streaming processes on sediment transport predictions. Section 6 summarizes the major conclusions of this study.

\subsection{MODEL FORMULATION}

\subsubsection{Equations describing the wave boundary layer}

This study considers the water motion under waves close to the bed to determine the net, period averaged current. The short period of the horizontal oscillation confines the generation of timedependent turbulence to a layer that is thin compared to the wave length. Therefore, the boundary layer approximation is applied and the flow field is described with a Reynolds-averaged momentum equation and a continuity equation:

$$
\begin{gathered}
\frac{\partial u}{\partial t}+u \frac{\partial u}{\partial x}+w \frac{\partial u}{\partial z}=-\frac{1}{\rho} \frac{\partial p}{\partial x}+\frac{\partial}{\partial z}\left\{\left(v+v_{t}\right) \frac{\partial u}{\partial z}\right\} \\
\frac{\partial u}{\partial x}+\frac{\partial w}{\partial z}=0
\end{gathered}
$$

where $u$ is the horizontal velocity, $w$ the vertical velocity, $\rho$ the density of water, $p$ the pressure, $v$ the kinematic viscosity of water, $v_{\mathrm{t}}$ the turbulent viscosity, $t$ the time and $x$ and $z$ horizontal and vertical axes directed respectively onshore and upward. Within the boundary layer, the horizontal pressure gradient is approximately constant over the vertical.

A $k$ - $\varepsilon$ model [Launder and Spalding, 1972; Rodi, 1984] provides the closure for $v_{\mathrm{t}}$ :

$$
\begin{gathered}
v_{t}=c_{\mu} \frac{k^{2}}{\varepsilon} \\
\frac{\partial k}{\partial t}+u \frac{\partial k}{\partial x}+w \frac{\partial k}{\partial z}=\frac{\partial}{\partial z}\left\{\left(v+\frac{v_{t}}{\sigma_{k}}\right) \frac{\partial k}{\partial z}\right\}+P_{k}-\varepsilon \\
\frac{\partial \varepsilon}{\partial t}+u \frac{\partial \varepsilon}{\partial x}+w \frac{\partial \varepsilon}{\partial z}=\frac{\partial}{\partial z}\left\{\left(v+\frac{v_{t}}{\sigma_{\varepsilon}}\right) \frac{\partial \varepsilon}{\partial z}\right\}+\frac{\varepsilon}{k}\left(c_{1 \varepsilon} P_{k}-c_{2 \varepsilon} \varepsilon\right)
\end{gathered}
$$

where $k$ is the turbulent kinetic energy, $P_{\mathrm{k}}$ is the turbulence production, $\varepsilon$ is the dissipation rate, and $\sigma_{\mathrm{k},} \sigma_{\varepsilon,} c_{\mu}, \mathrm{c}_{1 \varepsilon}$ and $c_{2 \varepsilon}$ are constants, respectively 1.0, 1.3, 0.09, 1.44, 1.92 (standard values), [Rodi, 1984]. The production term yields:

$$
P_{k}=v_{t}\left(\frac{\partial u}{\partial z}\right)^{2}
$$


because it follows from the boundary layer assumption that the contribution by vertical shear can be neglected. (Note that also sediment-induced stratification effects are not considered in the present hydrodynamic study).

\subsubsection{Forcing}

Two alternatives have been formulated to force the model. In the first alternative, here called the 'match' model, the principally unknown $u(z)$ is forced to match a predefined horizontal velocity signal at a certain vertical level $z_{\mathrm{m}}$. This level may be in, or a limited distance above, the wave boundary layer and the signal could have a non-zero mean. The associated pressure gradient is determined automatically by the model. In the second alternative, the 'free' model formulation, the unsteady horizontal pressure gradient $\tilde{p}$ is determined in advance from a given horizontal (component of a) free stream velocity $\tilde{u}_{\infty}$ with zero mean using:

$$
-\frac{1}{\rho} \frac{\partial \tilde{p}}{\partial x}=\frac{\partial \tilde{u}_{\infty}}{\partial t}+\tilde{u}_{\infty} \frac{\partial \tilde{u}_{\infty}}{\partial x}
$$

In this approach the net current arising from the streaming mechanisms is not compensated by any mean pressure gradient and is allowed to develop freely. The first alternative is especially suitable to compare the model with measurements that, by their nature, not only include boundary layer streaming mechanisms, but also possible return currents. The mere balance between boundary layer streaming mechanisms can be investigated using the second forcing alternative, adopting any temporal velocity series to predefine $\tilde{u}_{\infty}$, e.g. from $2^{\text {nd }}$ order Stokes theory (as applied by [Trowbridge and Madsen, 1984b; Holmedal and Myrhaug, 2009]). Second order Stokes theory gives:

$$
\hat{u}_{1, \infty}=\frac{\omega}{k} \frac{a k}{\sinh (k h)} \quad ; \quad \hat{u}_{2, \infty}=\hat{u}_{1, \infty} \frac{3}{4} \frac{a k}{\sinh ^{3}(k h)} \quad ; \quad \tilde{u}_{\infty}(t)=\sum_{n=1}^{2} \hat{u}_{n, \infty} \cos (n \omega t)
$$

with $\hat{u}_{\mathrm{n}, \infty}$ the amplitude of the $\mathrm{n}$-th harmonic component of $\tilde{u}_{\infty}, h$ the water depth and $k, a$ and $\omega$ respectively the wave number, amplitude and angular frequency.

\subsubsection{DV-approach}

If time- and length scale of changes in the wave shape are large compared to wave period and length, the wave can be considered as a sum of steady harmonic oscillations with identical phase speed. This allows for a $1 \mathrm{DV}$-approach by transforming horizontal velocity gradients into time derivatives [Trowbridge and Madsen, 1984b] with:

$$
\frac{\partial u}{\partial x}=-\frac{1}{c} \frac{\partial u}{\partial t}
$$

where $c$ is the wave celerity determined from water depth $h$ and wave period $T$ through the regular dispersion relation. Using transformation (2.9) and continuity equation (2.2) the vertical velocity at level $z$ can be expressed as: 


$$
w(z)=\frac{1}{c} \int_{0}^{z} \frac{\partial u}{\partial t} d z
$$

\subsubsection{Boundary conditions}

To solve equation (2.1), (2.4) and (2.5) using the 1DV-approach, six boundary conditions are needed. In the present model, the lower boundary $(z=0)$ is defined at the top of the roughness elements and turbulent flow over a hydraulically rough bed is modeled with a partial slip condition. Making use of an assumed logarithmic velocity profile close to the bed, the quadratic friction law, and local equilibrium between production and dissipation:

$$
\frac{u(z)}{u_{*}}=\frac{1}{\kappa} \ln \left(\alpha+\frac{z}{z_{0}}\right) \quad ; \quad u_{*}{ }^{2}=\left.\left(v_{t} \frac{\partial u}{\partial z}\right)\right|_{z=0} \quad ;\left.\quad P_{k}\right|_{z=0}=\left.\varepsilon\right|_{z=0}
$$

the lower boundary conditions are:

$$
\left.\frac{\partial u}{\partial z}\right|_{z=0}=\frac{u_{*}}{\alpha \kappa z_{0}} \quad ;\left.\quad k\right|_{z=0}=\frac{u_{*}^{2}}{\sqrt{c_{\mu}}} \quad ;\left.\quad \varepsilon\right|_{z=0}=\frac{u_{*}^{3}}{\alpha \kappa z_{0}}
$$

Where $u *$ is the friction velocity, $\kappa$ is the Von Karman constant, 0.41 , and $z_{0}$ is the roughness length scale for hydraulically rough flow related to the Nikuradse roughness height $k_{\mathrm{N}}$ with $z_{0}=k_{\mathrm{N}} / 30$. With $z=0$ defined at the top of the roughness elements, a value $\alpha \neq 1$ has to be adopted. Here, $\alpha=9$ is used based on Hinze [1975] and Jackson [1981].

In the free model formulation, no wave-averaged pressure gradient resulting from a mean surface slope caused by mass transport or radiation stress gradients is included. Therefore, shear and all vertical gradients in turbulence properties will be confined to the wave boundary layer, resulting in upper boundary conditions:

$$
\left.v_{t} \frac{\partial u}{\partial z}\right|_{z=t o p}=0 \quad ;\left.\quad \frac{\partial k}{\partial z}\right|_{z=t o p}=0 \quad ;\left.\quad \frac{\partial \varepsilon}{\partial z}\right|_{z=t o p}=0
$$

being applicable for a domain size exceeding the boundary layer thickness. In the match model formulation, application of these conditions is very well justified for comparison with experiments in oscillating flow tunnels in combination with a domain size that is half the tunnel height, forming a frictionless rigid lid acting as a line of symmetry. One could argue that these conditions are less suitable for simulation of net boundary layer currents from flume experiments, because the conditions slightly incorrectly assume no transfer of momentum by shear stress at the upper boundary. This may introduce possible model sensitivity to the domain height $Z$. However, sensitivity tests show that the dependency of the mean current for $Z$ vanishes for $Z$ is larger than around two and a half times the boundary layer thickness (tests not shown here). 


\subsubsection{Relation to other numerical boundary layer models}

The order of the advective terms in equation (2.1), (2.4) and (2.5) ( $2^{\text {nd }}$ and $3^{\text {rd }}$ term) compared to the others is $\mathrm{O}\left(\hat{u}_{\infty} / c\right)$. For waves in prototype situation, $\mathrm{O}\left(\hat{u}_{\infty} / c\right)$ is generally smaller than 0.3 . Although the unsteady horizontal flow in the wave boundary layer can be predicted rather well with only the terms of $O(1)$, it is essential to include these term to study the net current under waves, because the progressive wave streaming is driven by the (non-zero) wave averaged vertical advective transport of horizontal momentum into the wave boundary layer. With these terms turned off, free surface effects are neglected and our present model (called BL2-model) reduces again to the first order tunnel version (BL1-model) as used by [Ruessink et al., 2009] and [Hassan and Ribberink, 2010]. The BL-2 model can be considered as a representative RANS-boundary layer model with $k-\varepsilon$ turbulence formulations that includes the non-linear advective terms. It has strong similarities with the numerical models of Henderson et al. [2004] and Holmedal and Myrhaug, [2009], although there are small differences in the forcing and in the bed boundary condition (see Table 2.1 for an overview of model characteristics). Table 2.1 also shows the type of model validation carried out with these models so far, showing that the non-linear second-order models (BL2-type) are still lacking a validation with detailed velocity data measured in the wave boundary layer under controlled conditions. Information on the numerical solution method as applied in BL-1 and BL-2 can be found in appendix $\mathrm{C}$.

\subsection{VALIDATION}

\subsubsection{Test cases}

We validate the model with measurements of period-averaged and time-dependent horizontal velocities from laboratory experiments on boundary layer flow. Because of our focus on the hydrodynamics, we use fixed bed experiments. Four cases with various flow conditions have been selected, to cover situations with the various types of streaming both in isolation and combination.

Table 2.2 lists the four selected test cases. CASE 1 comes from small scale flume experiments in the Delft Scheldt Flume by Klopman [1994], with free surface waves with relatively small amplitudes ( $T=1.44 \mathrm{~s}, a=0.06 \mathrm{~m}, h=0.50 \mathrm{~m})$ and therefore nearly linear, sinusoidal form. With velocity skewness nearly absent, we expect the net current to be determined by progressive wave streaming and a return current only, the latter compensating for streaming induced mass transport and Stokes' drift in the closed facility. CASE 2 and 3 stem from experiments in the Aberdeen Oscillating Flow Tunnel by respectively Campbell et al. [2007] and [Van der A et al., 2011]. In tunnel experiments, the vertical component of the orbital velocity and therefore progressive wave streaming is absent. In CASE 2 , with velocity-skewed oscillatory flow, the mean current is determined by the offshore velocityskewness streaming and a return current that follows from the restriction of zero net mass transport in the closed tunnel facility. Also in CASE 3, we expect an offshore streaming, but now originating from the acceleration skewness of the flow [see Fuhrman et al., 2009a]. Although in accelerationskewed flows maximum on- and offshore horizontal velocity are equally large, differences in turbulence will still be present between on- and offshore half cycle, due to differences in 
development time of the boundary layer. CASE 4 originates from small scale flume experiments by Van Doorn [1981], with free surface waves with relatively large amplitudes in relatively small water depths $(T=2.0 \mathrm{~s}, a=0.052 \mathrm{~m}, h=0.30 \mathrm{~m})$. Under such circumstances, non-linear wave shapes will develop with increased wave crests and decreased and stretched wave troughs. This is expected to result in a combination of velocity-skewness streaming and progressive wave streaming inside the boundary layer. So, where the period-averaged velocity in CASE 1 to 3 arises from only one streaming mechanism (in combination with a return current), the net current in CASE 4 is generated by a combination of streaming mechanisms.

More information on the experimental facilities, the way the bed has been roughened and the method of velocity measurement in the various experiments can be found in Table 2.2. Note that in all experiments horizontal and vertical velocity components were measured in the vertical symmetry plane along the length axis of the facility. In CASE 4, measurements were taken in a vertical line above (V00RA) and in between (V00RB) the bed roughness elements.

\subsubsection{Note on flow regimes}

Starting from the assumption that the energetic waves in case of sheet-flow sediment transport in prototype situation generate turbulent flow, the model has been formulated for turbulent flow over a hydraulically rough, but flat bed. Before discussing the validation results, we investigate whether the flow in the validation cases might also be considered as a turbulent flow over a rough but flat bed. Firstly, note that the tunnel experiments aim for a one-to-one reproduction of the prototype situation, while the length scales of the waves in the small scale flume experiments of CASE 1 and 4 relate with approximately 1:10 to prototype length scales. Although this scaling will not influence the wave dispersion, the boundary layer flow might be affected. Orbital flow velocity and excursion $\hat{u}_{1}$ and $A$ will be much smaller, causing a reduction of the (wave) Reynolds number $R e=\hat{u}_{1} A / v$ (with factor $10^{1.5}$ for mentioned scaling). For lower Reynolds numbers, turbulent flow can only be generated with larger (relative) bed roughness. Figure 2.1 shows the position of the experiments in a chart of the flow regimes as determined by the non-dimensional relative roughness $A / k_{\mathrm{N}}$ and the nondimensional wave Reynolds number $R e$, with $k_{\mathrm{N}}$ the Nikuradse roughness height (values as discussed in section 2.3.3). The regime delineations are gathered from Jonsson [1966], Jonsson [1980], Davies [1980], Fredsøe and Deigaard [1992] (fig. 2.13) and Davies and Villaret [1999].

Figure 2.1 shows that CASE 3 is at the border of the rough turbulent flow regime. The scaled flume experiment of CASE 4 is in the very rough turbulent regime. In this case, the relative large roughness elements might even cause 2D-effects [Davies and Villaret, 1999], not accounted for in the flat bed model approach. Despite the large roughness, CASE 1 is situated just inside the transition from turbulent to laminar. Apparently the wave, with small amplitude to maintain linearity, was too small to generate fully turbulent boundary layer flow. Also CASE 2 is just outside the rough turbulent flow regime. Here, the roughness elements are of the same order of magnitude as the viscous sub-layer $\delta_{\mathrm{v}}$, and the flow tends to be of turbulence in hydraulically smooth conditions. The line of $\delta_{\mathrm{v}} / k_{\mathrm{N}}=1$, the 
dash-dotted line in Figure 2.1, has been estimated from conventional expressions for friction velocity $u_{*}$ in steady flow [Fredsøe and Deigaard, 1992; see also Justesen, 1988] (here $\alpha=11.7$ ):

$$
\delta_{v}=\alpha \frac{v}{u_{*}} ; \quad u_{*}=\sqrt{0.5 f_{w}} \hat{u}_{1, \infty} ; \quad f_{w}=\frac{2}{\sqrt{\operatorname{Re}}} ; \quad \rightarrow \quad \frac{\delta_{v}}{k_{N}}=\alpha \operatorname{Re}^{-3 / 4} \frac{A}{k_{N}}
$$

with $f_{\mathrm{w}}$ the friction factor.

In this study, we use CASE 1 to 4 for validation of the rough turbulent boundary layer model. Some influence of changing $R e$ is included in the model (see equation (2.1)). Alternative formulations for the smooth turbulent or transitional regime and their influence on streaming are not explored in the present study.

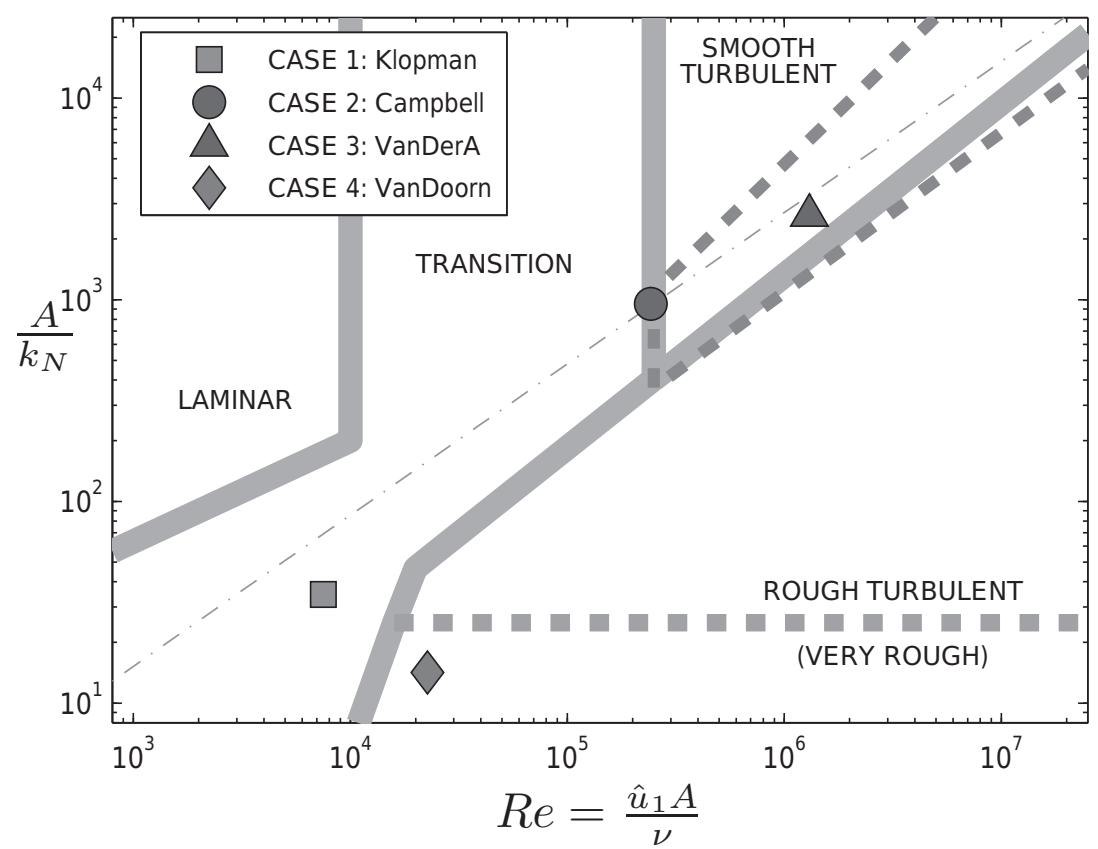

Figure 2.1: Delineation of flow regimes with position of validation cases, following the example of Davies [1980]. Thick gray lines based on Jonsson [1966] and Jonsson [1980]. Thick dashed dark gray line: transition zone from rough turbulent to smooth turbulent as derived from Fredsøe and Deigaard [1992, fig 2.13]. Thick dashed light gray line: transition from rough to very rough turbulent flow, with $2 \mathrm{D}$ effects around roughness elements (e.g. ripples) from Davies and Villaret [1999]. Thin dash-dotted line: line of $\delta_{\mathrm{v}} / k_{\mathrm{N}}=1$, an indication for the rough-smooth transition. 


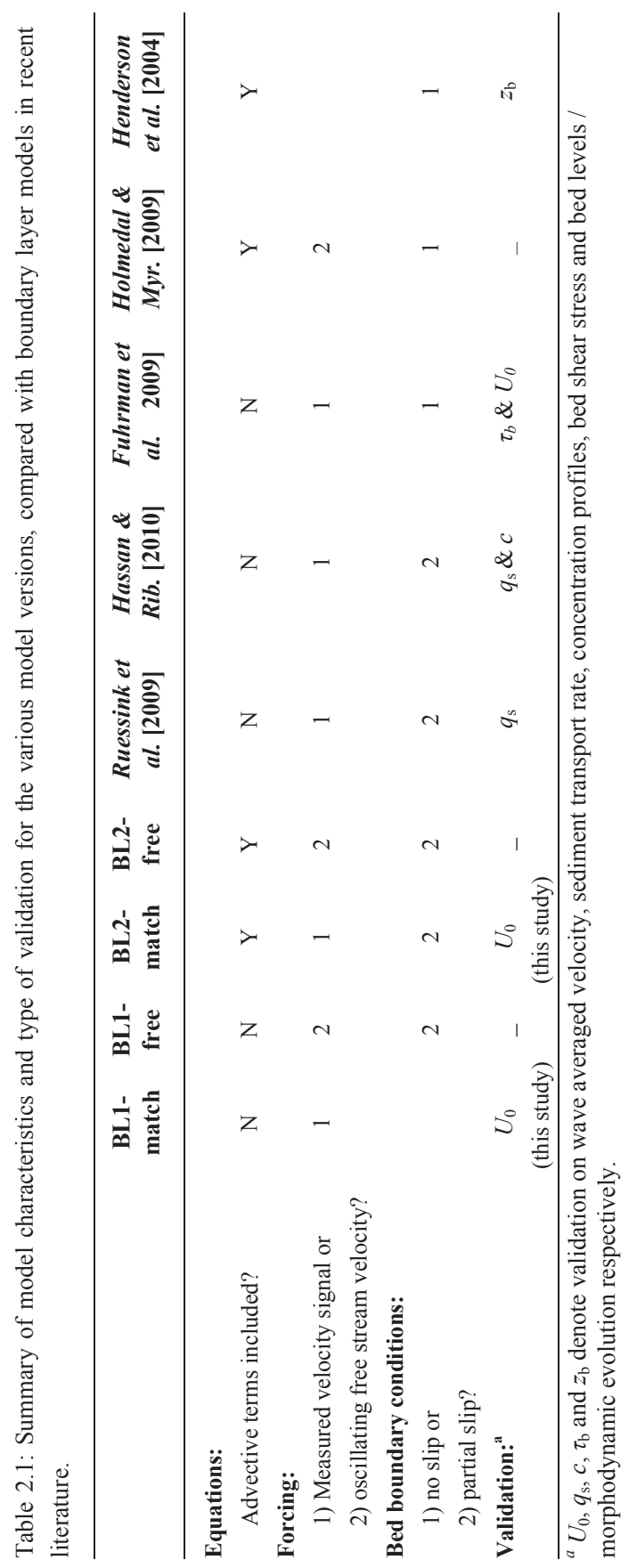




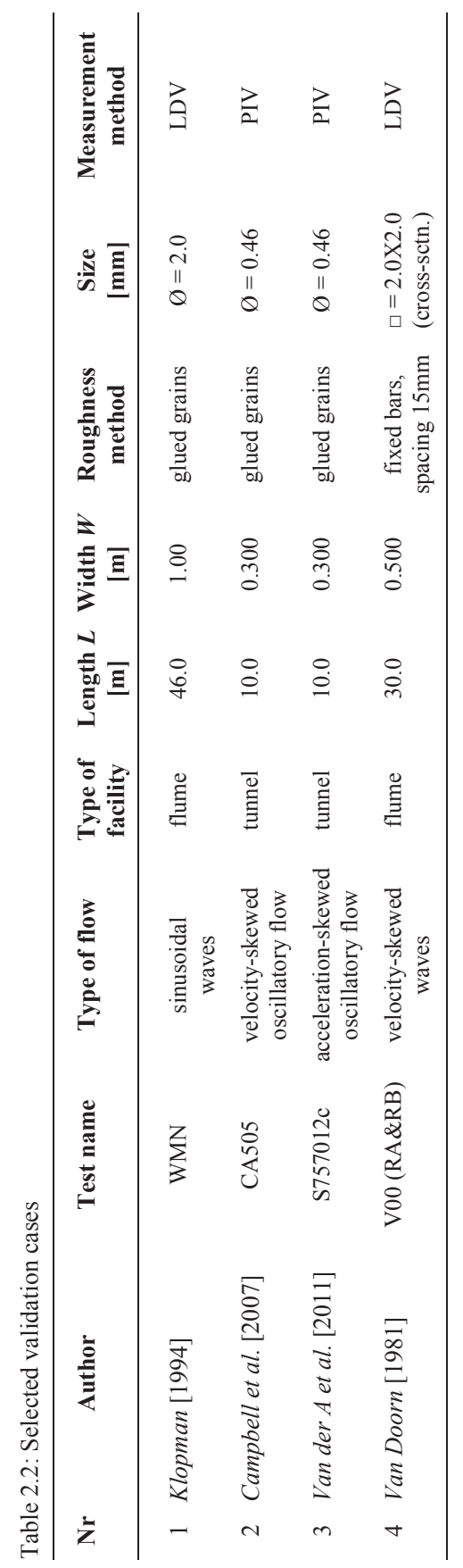

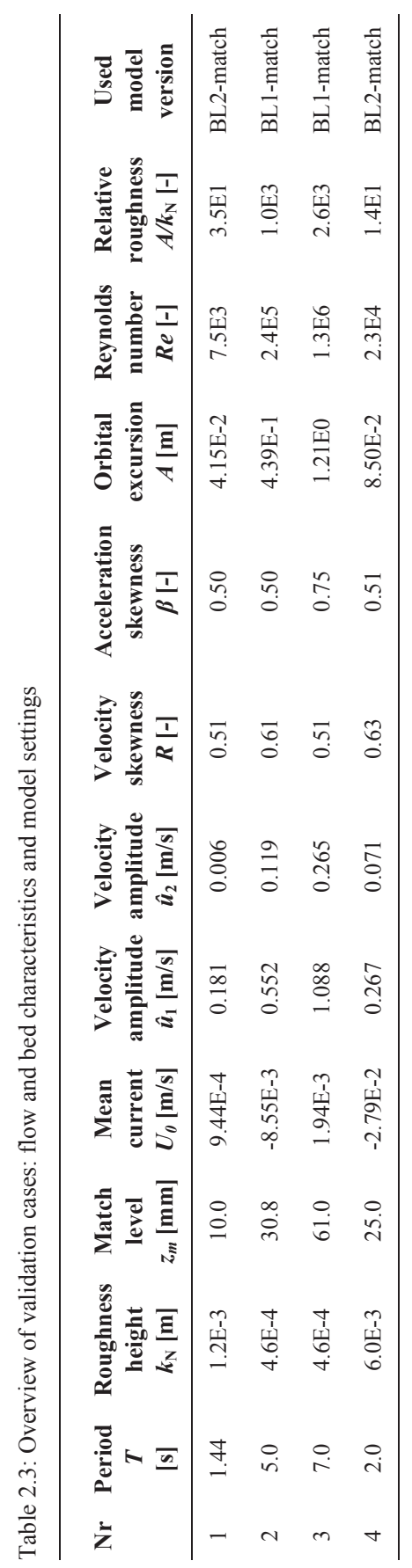




\subsubsection{Simulation set-up}

In order to force the model in a consistent way for the different test cases, we force the model to match the measured velocity signal at the measurement location closest to $2.5^{*} \delta_{\mathrm{s}}$, with $\delta_{\mathrm{s}}$ the boundary layer thickness estimate of [Sleath, 1987]:

$$
\frac{\delta_{s}}{k_{N}}=0.27\left(\frac{A}{k_{N}}\right)^{0.67}
$$

and $A$ the orbital excursion. Note that with the selection of a high matching level $z_{\mathrm{m}}$, a large degree of freedom is allowed for the net current inside the boundary layer, which yields a more conclusive model validation. However, selection of a high matching level also has disadvantages: the measurements could be affected by side wall effects, non-hydrostatic pressure effects or other processes absent in the model. The present choice for $z_{\mathrm{m}}$ yields a consistent treatment of all validation cases and takes account of the mentioned considerations. The model domain size is set to five times the matching level. Another modeling choice concerns the bed roughness height. In CASE 1 we use a Nikuradse roughness height $k_{\mathrm{N}}=1.2 \mathrm{~mm}$ as derived by Klopman [1994] from experiments with current only. In the other validation cases we use $k_{\mathrm{N}}=\gamma d_{50}$, with $\gamma$ somewhere between 1 and 3 , the exact value chosen based on the best fit of computed and measured level of maximum amplitude of the first harmonic component of the time dependent signal $\left(\hat{u}_{1}\right)$. The used values for $z_{\mathrm{m}}$ and $k_{\mathrm{N}}$ can be found in Table 2.3, together with information on the input velocity signal, characterized by velocity-skewness and acceleration-skewness parameters $R$ and $\beta$, with:

$$
R=\frac{u_{\infty \text { max }}}{u_{\infty \text { max }}+\left|u_{\infty \text { min }}\right|} ; \quad \beta=\frac{\dot{u}_{\infty, \max }}{\dot{u}_{\infty, \max }+\left|\dot{u}_{\infty, \min }\right|}
$$

where $u_{\infty}$ and $\dot{u}_{\infty}$ are the velocity and acceleration of the free stream respectively.

\subsubsection{Validation results}

The results are presented in Figure 2.2 and Figure 2.3. These figures show the mean $U_{0}$ and the amplitude $\hat{u}$ and phase $\theta$ of the harmonic components of the measured and computed horizontal velocity $u(z, t)$, where:

$$
u(z, t)=U_{0}(z)+\sum_{n=1}^{n_{\max }} \hat{u}_{n}(z) \cos \left\{n \omega t+\theta_{n}(z)\right\}
$$

Firstly, we consider the time-dependent flow, focusing the model-data comparison on the features boundary layer thickness, phase lead and velocity overshoot. The phase lead, increasing with decreasing distance to the bed, and the velocity overshoot, an increased maximum orbital velocity just inside the wave boundary layer, (both compared to the free stream), are visible in panels $\mathrm{b}$ and $\mathrm{c}$ of Figure 2.2 and Figure 2.3, that show respectively amplitude $\hat{u}_{1}$ and phase $\theta_{1}$ of the first harmonic component. As proxy of the thickness, we look to the level where $\hat{u}_{1}$ has its maximum. Note that this 
feature was actually used for a slight tuning of roughness $k_{\mathrm{N}}$. We observe from the figures' panels (b) and (c) that the velocity overshoot and phase lead are present in the model results for $\hat{u}_{1}$ and phase $\theta_{1}$ in all cases. For CASE 2 to 4 , the data also show a velocity overshoot in $\hat{u}_{2}$ and $\hat{u}_{3}$ (respectively panel $\mathrm{d}$ and $\mathrm{f}$ ) and a phase lead in $\theta_{2}$ and $\theta_{3}$ (respectively panel e and g). These features are also present in the model results. We especially point at the neat reproduction of the local minimum and two velocity overshoots in $\hat{u}_{3}$ of CASE 3 with acceleration-skewed flows. Note that for this case the third harmonic is more important than in the other cases (compare $\hat{u}_{3} / \hat{u}_{1}$ ). In aid of further model skill assessment, Table 2.4 provides quantitative measures of the reproduction quality: $S\left(\hat{u}_{\mathrm{n}, \max }\right)$ is the ratio of maximum amplitude for component $n$ in model and data, $S\left(\theta_{\mathrm{n}, \mathrm{z}=\mathrm{min}}\right)$ is the ratio of modeled and measured phase lead for component $n$ at the lowest data point. Figures and table show that $0.8<\mathrm{S}<1.2$ for most of the cases. For CASE 2 and especially 4 the near-bed phase lead is predicted somewhat worse. We briefly return on explanations of this mismatch in section 2.3.5 and on implications hereof for further model application in section 2.5 .
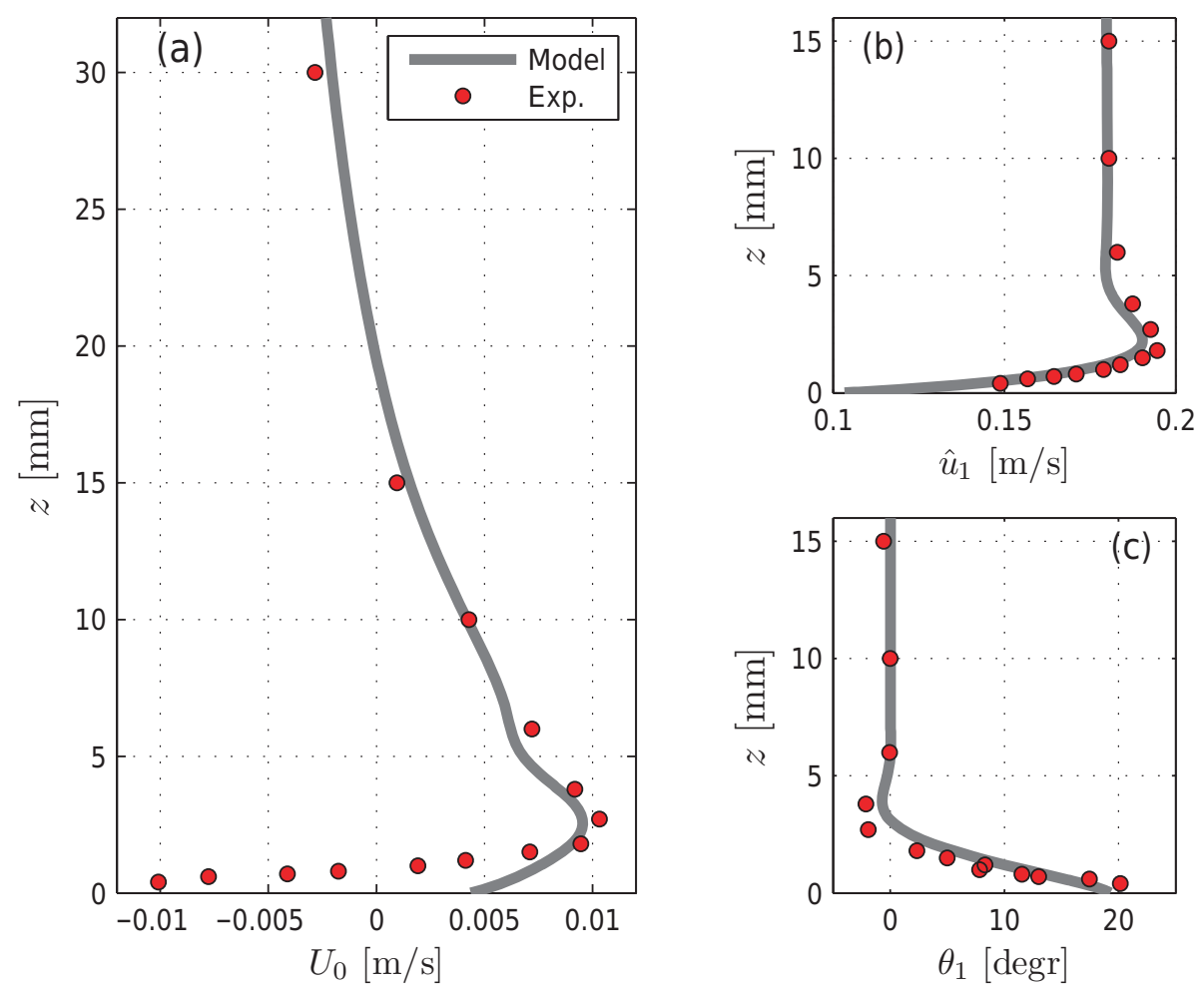

Figure 2.2: Model-data comparison on (a) wave averaged velocity $U_{0}$ and (b) amplitude $\hat{u}$ and (c) phase $\theta$ of $1^{\text {st }}$ harmonic component of the horizontal velocity for validation CASE 1: Klopman (see Table 2.2 and Table 2.3). Positive velocities in (a) are directed onshore. 

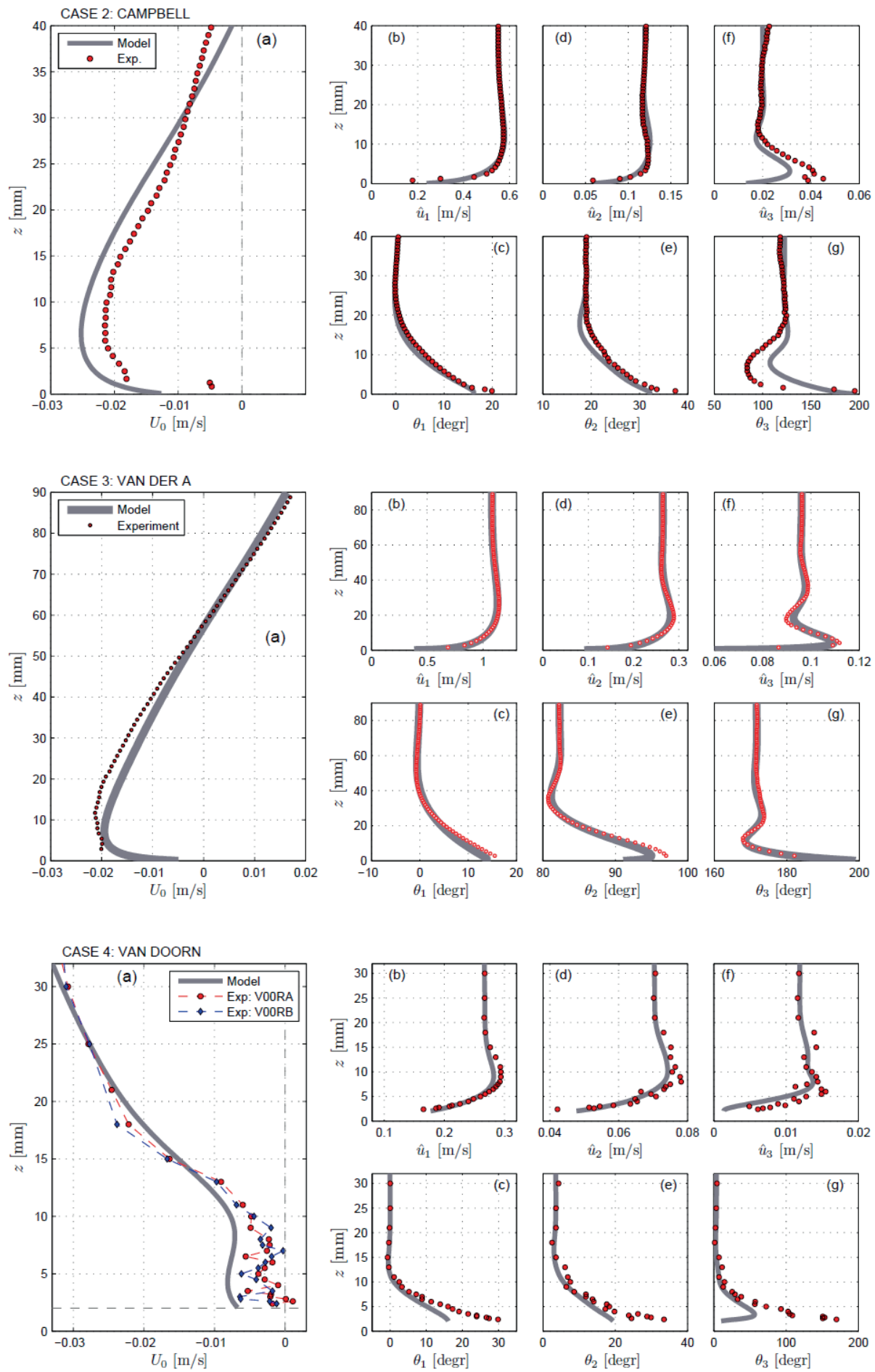
Figure 2.3 (previous page): Model-data comparison on (a) wave averaged velocity $U_{0}$ and (b, d, f) amplitudes and (c, e, g) phases of the first three harmonic velocity components as function of $z$ for validation CASE 2, 3 and 4 (see Table 2.2 and Table 2.3). Positive values in (a): onshore directed velocities.

Next, we consider the period averaged horizontal current velocity $U_{0}$, comparing model and data for direction, magnitude and shape of the streaming profile. The quality of the reproduction is again quantified in Table 2.4 with $S\left(\left|U_{0}\right|, \max \right)$ the ratio of maximum absolute streaming velocity inside the boundary layer in model and data, and $S\left(z\left(\left|U_{0}\right|, \max \right)\right)$ the ratio of the level of maximum streaming in model and data. For CASE 1 to 3, direction and profile shape of $U_{0}$ are correctly reproduced by the model. Consistent with the expected dominance of progressive wave streaming beneath a linear wave, the results in CASE 1 show an onshore current inside the wave boundary layer, even though the mean pressure gradient generates an offshore current. The absence of a negative horizontal velocity component in the model results close to the bed is not considered as a major defect. It should be noted that in this scaled flume experiment the grains $(d \approx 2 \mathrm{~mm})$ were very large compared to the wave boundary layer thickness (approximately 6mm). Therefore, Klopman [1994, p.33] attributed these negative horizontal velocity components to the local effect of individual sand grains at the particular horizontal position where the measurements were taken. For CASE 2 and 3, both data and model show an offshore boundary layer current, consistent with the expected wave shape streaming for both velocity-skewed and acceleration-skewed oscillatory flow. We observe in Figure 2.3 for CASE 2 a clear overestimation of $\left|U_{0, \max }\right|$ and, like in the time-dependent flow for this case, a mismatch in the 3 to 4 lowest measurement locations. On the other hand, $U_{0}$ is neatly reproduced in CASE 3. This is an important result, because earlier effort to reproduce the (direction of the) mean flow in acceleration-skewed oscillations using an analytical boundary layer model [Gonzalez Rodriquez, 2009, fig. 4-14] (basically the model of Trowbridge and Madsen [1984b] with adapted upper boundary conditions to account for return currents in the closed facility) was not successful. An analysis of the contributions from the various harmonic components to the mean shear stress showed that the contribution of the 3rd harmonic components of eddy viscosity and horizontal velocity was significant. These components were not included in the analytical models. We therefore believe that the success of the present model to reproduce offshore streaming in acceleration skewed flow is essentially because the model includes the higher harmonic components.

Table 2.4: Quantification of the reproduction quality by model/data ratios

\begin{tabular}{|c|c|c|c|c|c|c|c|c|}
\hline \multirow[b]{2}{*}{ CASE } & \multicolumn{3}{|c|}{ Amplitude } & \multicolumn{3}{|c|}{ Phase lead } & \multicolumn{2}{|c|}{ Mean } \\
\hline & $S\left(\hat{u}_{1, \max }\right)$ & $S\left(\hat{u}_{2, \max }\right)$ & $S\left(\hat{u}_{3, \max }\right)$ & $S\left(\theta_{1, \mathrm{z}=\mathrm{min}}\right)$ & $S\left(\theta_{2, z=\min }\right)$ & $S\left(\theta_{3, z=\min }\right)$ & $S\left(\left|U_{0}\right|_{\text {,max }}\right)$ & $S\left(z\left(\mid U_{0 \mid, \max }\right)\right)$ \\
\hline 1 & 0.98 & not rel. ${ }^{\mathrm{a}}$ & not rel. ${ }^{\text {a }}$ & 0.94 & not rel. ${ }^{\text {a }}$ & not rel. ${ }^{a}$ & 0.93 & 0.94 \\
\hline 2 & 1.01 & 1.02 & 0.72 & 0.80 & 0.70 & 0.86 & 1.16 & 0.93 \\
\hline 3 & 0.99 & 0.97 & 0.97 & 0.88 & 0.88 & 1.00 & 0.92 & 0.67 \\
\hline 4 & 0.96 & 0.95 & 0.89 & 0.53 & 0.58 & 0.06 & $+/-2$ & - \\
\hline
\end{tabular}

a) not relevant 


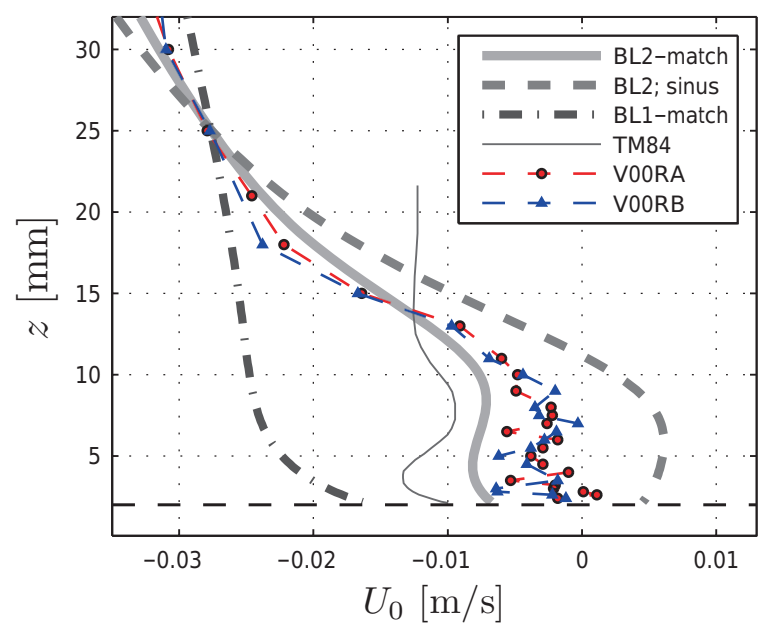

Figure 2.4: Period averaged velocity $U_{0}$ computed with: 1) complete model (BL2-match model, solid curve); 2) velocity-skewness streaming excluded (BL2-sinus, dashed line); 3) progressive wave streaming excluded (BL1-match model, dash-dotted line), compared with measurements of Van Doorn [1981] in the vertical above (V00RA) and in between (V00RB) the roughness elements, and the analytical results of Trowbridge and Madsen [1984b] (TM84, thin solid line). Positive velocities are onshore directed. All model simulations are forced at $z=25 \mathrm{~mm}$.

For CASE 4 the (negative) streaming is clearly overpredicted by the model, however it should be realized that the measured mean velocities near the bed are very close to zero and show a relatively large scatter. The absolute magnitude of the overprediction is only a few $\mathrm{mm} / \mathrm{s}$. Figure 2.4 shows that the present model clearly gives improved predictions compared to the analytical model of Trowbridge and Madsen [1984b] (TM84). The reason why we compare with this model is that it is essentially this model that has been used by Gonzalez Rodriquez and Madsen [2011] (GRM) to investigate the influence of streaming on sediment transport (medium sized sand). Note that the adapted boundary conditions of GRM compared to TM84, incorporating the negative return flow, will lead to an even worse analytical prediction for $U_{0}$ in CASE 4. Like TM84, the present model (BL2-match) shows a clear competition between the generation of offshore directed streaming close to the bed and onshore directed streaming at a higher levels inside the boundary layer (local minimum and maximum). Above $z=9 \mathrm{~mm}$, the $U_{0}$-profile bends in offshore direction: Within the boundary layer, both velocity-skewness streaming and progressive wave streaming are present, but keep each other (in this case) practically in balance, explaining measured net currents so close to zero. Outside the boundary layer, however, where these mechanisms are not active anymore, the return current is the dominating mechanism governing $U_{0}$. To illustrate this balance quantitatively, we add simulations to Figure 2.4 with the velocity-skewness respectively the progressive wave streaming mechanism turned off. The first is achieved by forcing the BL2-match model with mean and first harmonic only (a sinusoidal wave), the second by forcing the BL1-match model with the complete measured $u(z, t)$ at $z_{\mathrm{m}}$. The first predicts onshore streaming, while the latter predicts far too 
much offshore streaming. Both are clearly further off than the complete BL2-match model. The improved predictions of the present model compared to TM84 can probably be explained by the turbulence memory effect, as included in the k- $\varepsilon$ model: Turbulent kinetic energy generated by the strong onshore movement is diffused upward. Because this takes time, this t.k.e. can even end up in offshore directed flow, thus reducing the difference in turbulent shear stresses during on- and offshore flow and therefore also reducing the offshore velocity-skewness streaming component.

\subsubsection{General model behaviour}

To explain the underestimation of the phase lead in CASE 4, the behaviour of the model under influence of changing roughness has been investigated in more detail by studying the computed friction factor $f_{\mathrm{w}}$ and level of maximum velocity overshoot $z\left(\hat{u}_{1, \max }\right)$ for sinusoidal oscillations (so no streaming involved). It appears that inside the rough turbulent regime, model results for $f_{\mathrm{w}}$ and $z\left(\hat{u}_{1, \max }\right)$ are well described with:

$$
f_{w}=0.062\left(\frac{A}{k_{N}}\right)^{-0.3}, \quad \frac{z_{u_{1}, \max }}{k_{N}}=0.135\left(\frac{A}{k_{N}}\right)^{0.75}
$$

When we compare the model predicted $f_{\mathrm{w}}$ with (empirical) relations from literature (Figure 2.5), we observe that for $A / k_{\mathrm{N}}>50$ the relations from literature are reproduced rather well. However, for $A / k_{\mathrm{N}}$ $<50$ the friction is underpredicted. Such model performance for oscillatory flows has been observed before, see e.g. Justesen [1988] ( $k-\varepsilon$ turbulence closure) and Fuhrman et al. [2009a] (k- $\omega$ turbulence closure) and is ascribed to the fact that the model does not consider the 2-dimensional flow phenomenae taking place around the relatively large roughness elements in the very rough turbulent regime. An under predicted friction factor in CASE 4 would indeed explain the underestimation of the phase lead.

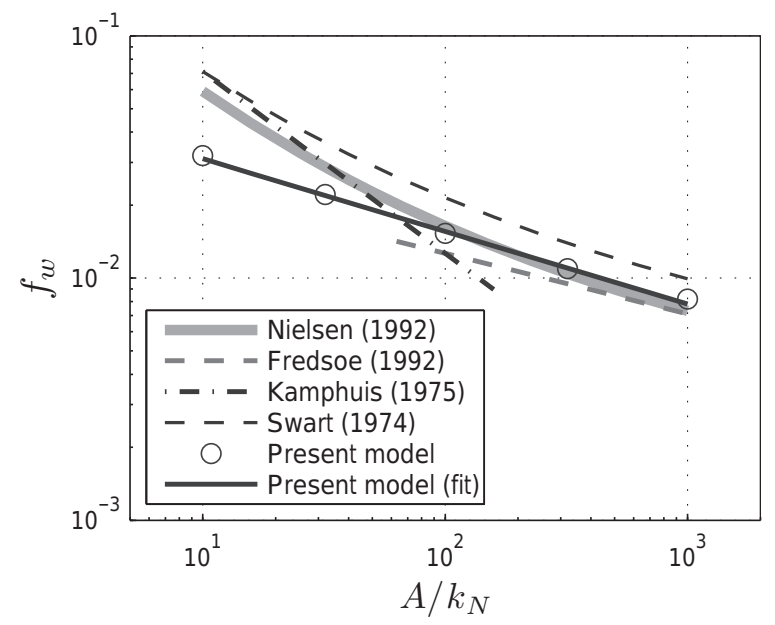

Figure 2.5: Wave friction factor $f_{\mathrm{w}}$ versus $A / k_{\mathrm{N}}$ from the present model compared to various (empirical) relations from literature. 


\subsection{ANALYSIS OF STREAMING GENERATING MECHANISMS}

We subsequently investigate how the observed direction and shape of the net current profiles can be attributed to the various streaming mechanisms and their potential competition. Next, we explore systematically how this competition will change for changing wave and bed conditions. Finally, we study the effects of the mean pressure gradient.

\subsubsection{Streaming mechanisms in the validation cases}

We use our model to assess and distinguish the influence of the various mechanisms on the $U_{0}$ profile. Firstly, a 'shape'-expression has been derived from the momentum balance (by period averaging and integration over $z$, see also appendix D, overbar indicates period-averaging):

$$
\rho\left(v+\bar{v}_{t}\right) \frac{\partial \bar{u}}{\partial z}=\rho\left(\overline{u w}-\overline{u w}_{\infty}\right)+\frac{\partial \bar{p}}{\partial x}(z-h)-\rho \tilde{\tilde{v}} \frac{\overline{\partial \tilde{u}}}{\partial z}
$$

This shows the influence of the various momentum transferring mechanisms to the mean velocity gradient (note that the wave averaged viscosity is always positive) or more precise the currentrelated part of the mean shear stress. The terms on the right hand side show respectively the contributions from 1) mean momentum transport by vertical velocity ('wave Reynolds stress') driving the progressive wave streaming, 2) the wave-averaged pressure gradient, and 3) differences in turbulence between the on- and offshore phase of the wave driving the wave shape streaming (wave-related mean shear stress). Secondly, profiles of all these terms have been computed from the model results. A direct comparison of the four validation cases is possible after normalization. The vertical distance has been scaled by $\delta^{*}$, an estimate for the thickness of the turbulent wave boundary layer [Nielsen, 1992; Swart, 1974]:

$$
\delta^{*}=\sqrt{\frac{f_{w}}{2}} A ; \quad f_{w}=\exp \left\{5.5\left(\frac{A}{k_{N}}\right)^{-0.2}-6.3\right\}
$$

The stress contributions are scaled by the maximum bed shear stress $\tau_{\mathrm{b}, \mathrm{m}}$ exerted by a sinusoidal oscillatory flow with a velocity amplitude $\hat{u}_{1 \infty}$ identical to the validation case [see Fuhrman et al., 2009a]. Here, this $\tau_{b, m}$ was obtained from simulations, but can equally well be computed with $\tau_{\mathrm{b}, \mathrm{m}}=1 / 2 \rho f_{\mathrm{w}} \hat{u}_{1}^{2}$ and $f_{\mathrm{w}}$ according to (2.18). These results are shown in Figure 2.6.

In Figure $2.6 \mathrm{a}, \mathrm{b}, \mathrm{c}, \mathrm{d}$ we observe the following (in order of increasing interest): No contribution of the wave Reynolds stress (b) is present in the tunnel cases (CASE 2 and 3). A positive mean pressure gradient (c) is present in the flume cases (CASE 1 and 4), a negative in the tunnel cases, consistent with the directions of the mass transport compensation currents. The wave shape related contribution (d) is negative for all cases, running from a maximum negative value at or very near the bed towards zero around $\delta^{*}$. This contribution is not only present for the velocity-skewed oscillations / waves (CASE 2 and 4), but also under the acceleration-skewed oscillation (CASE 3), albeit smaller. Also the practically linear wave in CASE 1 shows a negative wave shape contribution (d). We ascribe this to 
(a)

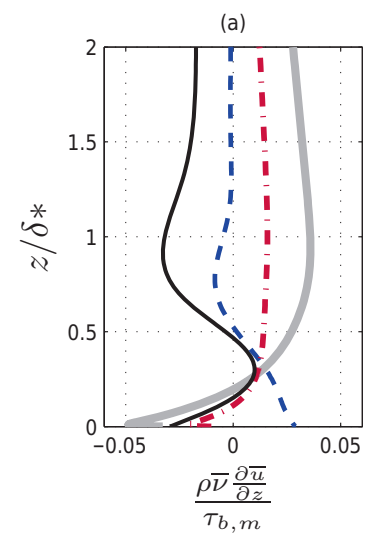

(b)

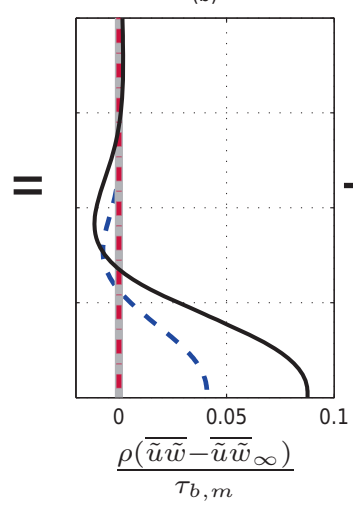

(c)

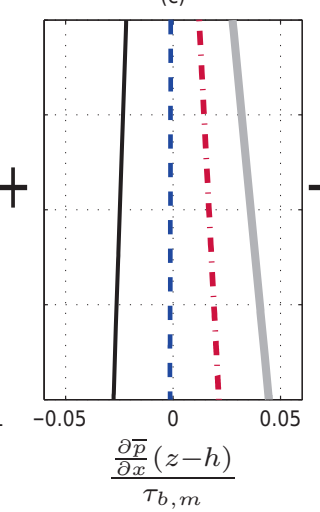

(d)

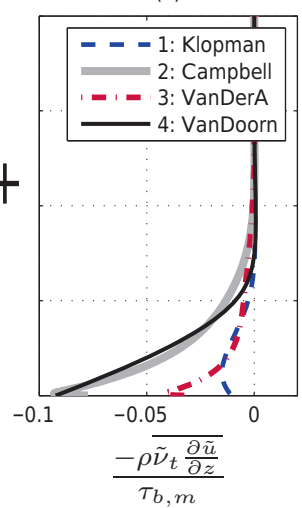

Figure 2.6: Normalized contributions to the current related mean shear stress (a) from the wave Reynolds stress (b), the mean pressure gradient (c) and the wave shape (d) for validation CASE 1 to 4 .

the increased onshore and reduced offshore near bed velocities due to the positive progressive wave streaming, introducing a turbulence behaviour like under velocity-skewed oscillation. The deviation of the wave Reynolds stress (b) from its free stream value has the same form in the two flume experiments: constant and positive close to the bed and subsequently twisting around zero with decreasing amplitude for increasing distance from the bed. For CASE 4, the summation in panel (a) shows a clear competition between the contribution from the wave Reynolds stress and from the wave-related mean stress. At low levels, apparently the wave shape streaming wins and the velocity gradient is negative. At higher levels, the gradient becomes positive and subsequently negative again, under influence of the progressive wave streaming mechanism. This explains the velocity profile in Figure 2.4, where the negative velocity is the result of velocity-skewness streaming, but the bulb in positive direction follows from the progressive wave streaming. Note that the latter has its level of maximum influence on a higher level than the first.

\subsubsection{Influence of changing wave and bed conditions}

Under free surface waves in prototype situation, both streaming phenomena act simultaneously. However, their contribution can vary largely with varying wave conditions. When waves approach the shore, orbital velocities close to the bed will increase while the wave propagation velocity decreases. Therefore, progressive wave streaming may be expected to increase with decreasing depth. However, the wave shape will change simultaneously. Where wave shape streaming due to velocity-skewness is absent for linear waves offshore, it will also increase with decreasing depth. So it is not a priory clear which of the streaming mechanisms wins. Earlier analytical investigation of this balance by Trowbridge and Madsen [1984b] revealed a reversal of the streaming velocity at the edge of the bottom boundary layer from on- to offshore for relatively long waves. Holmedal and Myrhaug's [2009] numerical simulations showed increasing importance of velocity-skewness streaming over progressive wave streaming for increasing wave periods, qualitatively consistent with Trowbridge and Madsen [1984b]. Here, we use the validated numerical model for a systematic 
quantitative investigation on the balance between the competing mechanisms for changing wave and bed conditions on the shoreface. These general insights in streaming are considered to be valuable for the development of adequate hydrodynamic input for practical sand transport formulae.

\subsubsection{The non-dimensional parameter domain}

The hydrodynamics of the boundary layer above a flat horizontal bed under a free surface wave is completely described by the parameters $a, h, T, k_{\mathrm{N}}, g$ and $v$. With six dimensional parameters and two fundamental dimensions, this situation can be described by combinations of four basically independent non-dimensional parameters, e.g. $a / h, k h, A / k_{\mathrm{N}}$ and $R e$, respectively the relative wave amplitude, relative water depth, relative bed roughness and the (wave) Reynolds number. Note that other informative non-dimensional parameters can be derived from these 4 parameters, for instance the parameter $\hat{u}_{1} / c$ that indicates the relative importance of the advective terms in the momentum equation (2.1), and the parameter $R$ that describes the degree of velocity-skewness (2.16). In contrast with tunnel experiments, velocity-skewness $R$ is not a free parameter under real free surface waves. It depends on the relative water depth $k h$ and relative wave amplitude $a / h$. To describe the shape of the near bed velocity signal as function of these parameters, a wave theory or model is needed. Using $2^{\text {nd }}$ order Stokes theory, see equation (2.8), $R$ can be expressed as:

$$
R=\frac{1}{2}+\frac{3}{8} \frac{a k}{\sinh ^{3}(k h)}
$$

From the four non-dimensional parameters $a / h, k h, A / k_{N}$ and $R e$, the first three are considered most relevant studying streaming and shear stress in a turbulent wave boundary layer potentially inducing sheet-flow: wave condition parameters $a / h$ and $k h$ give the forcing of the boundary layer model, parameter $A / k_{\mathrm{N}}$ directly influences the friction of the flow over the bed. Within the (rough) turbulent flow regime, the influence of $R e$ on the boundary layer flow characteristics should diminish. Extensive tests on model behavior do confirm this and show that the area of Re-independent model results coincides quite well with the experimentally determined delineation of the rough turbulent flow regime (see Figure 2.1). Restricting our exploration to this flow regime, we therefore couple the Re number to the relative roughness $A / k_{\mathrm{N}}$ with:

$$
\mathrm{Re}=1.75 * 223\left(\frac{A}{k_{N}}\right)^{1.17}
$$

which is a line parallel to the turbulent delineation of Jonsson [1966] in Figure 2.1, inside the rough turbulent regime.

We investigate the balance between velocity-skewness streaming and progressive wave streaming in the turbulent wave boundary layer for a domain spanned by the remaining parameters $a / h, k h$ and $A / k_{\mathrm{N}}$. Because we use second order Stokes theory to determine the oscillating free stream velocity (model input), cases outside the domain of applicability of this theory (wave breaking, too much non-linearity) have been excluded from the further procedure. The used restrictions are: $a / h<0.4$ and $R<0.625$ (coincides more or less with Ursell number $U=H L^{2} / h^{3}<45$, with $H$ and $L$ wave height 
and length respectively). See the delineation in the upper panel of Figure 2.7. Within these limits, cases have been defined (circles, same figure), and simulations have been carried out using the BL2free-model for zero mean pressure gradient. Following Trowbridge and Madsen [1984b], the computed streaming velocity just outside the boundary layer is taken as a measure in the visualization of the results. Dependency on $a / h$ is nearly completely removed from the visualization when the streaming is normalized as $\left(U_{0} / \hat{u}_{1}\right) /\left(\hat{u}_{1} / c\right)$. This can be seen from Figure 2.7 (lower panel), showing results for $A / k_{\mathrm{N}}=100$. Only at the outer edges of the domain, the surface formed by the numerical results is slightly bent in $a / h$ direction (which is attributed to slight numerical inaccuracies in the extreme cases). Note that the $a / h$ independency in the mentioned normalization reduces the normalized streaming to a function of $k h$ and $A / k_{\mathrm{N}}$ only.
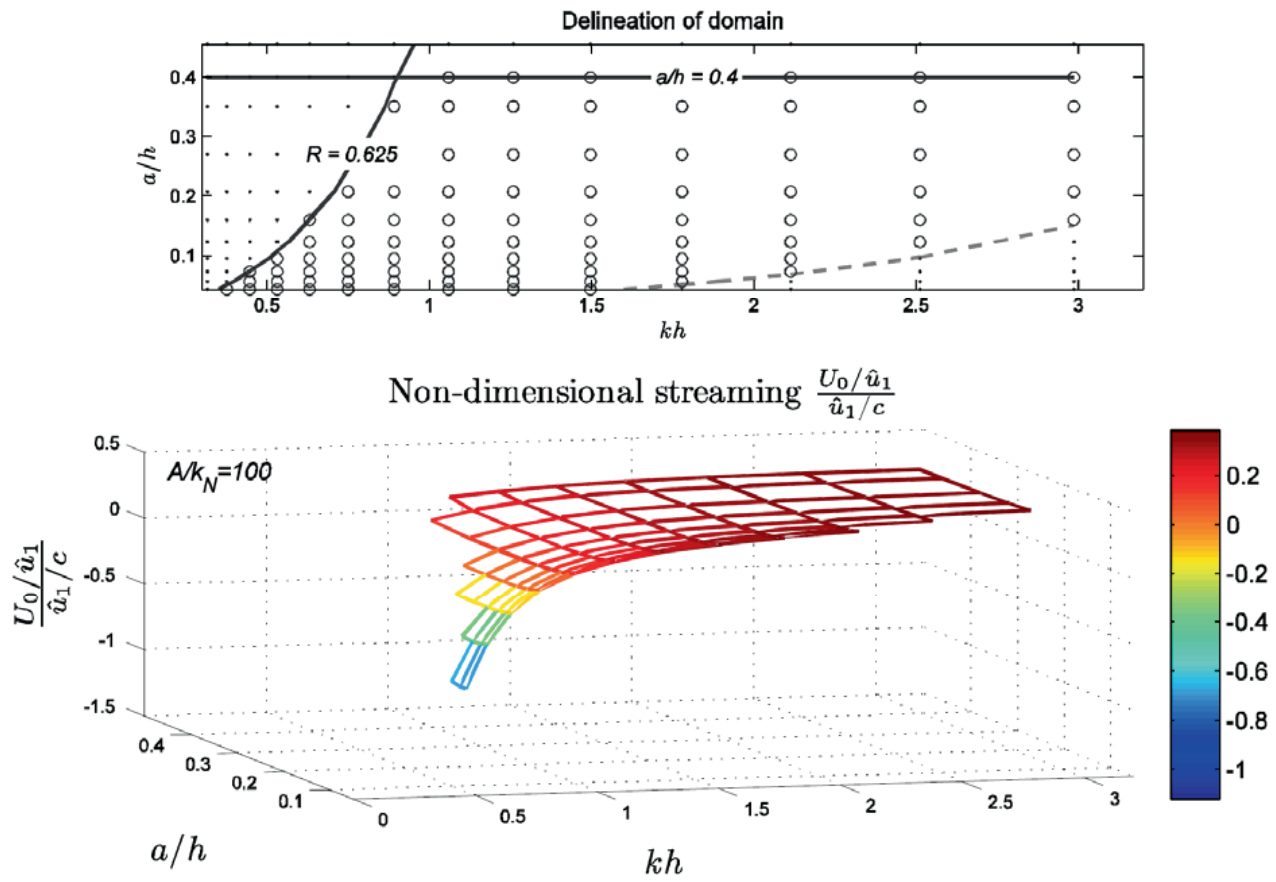

Figure 2.7: (top) Delineation of realistic parameter combinations in the plane spanned by $k h$ and $a / h$; (bottom) Non-dimensional streaming velocities at outer edge of the boundary layer as function of $k h$ and $a / h$ for $A / k_{\mathrm{N}}$ $=100$. 


\subsubsection{Influence of relative water depth $\mathrm{kh}$}

Figure 2.8 shows the non-dimensional streaming as function of $k h$ for a single roughness. The results show a clear dependence on $k h$ : streaming is positive at large $k h$, but decreases more and more for decreasing $k h$. Simulations with wave shape effect and progressive wave effect only, clarify these results: at relatively deep water (large $k h$ ) the non-dimensional streaming is completely determined by the free surface effect. For decreasing relative water depth $(k h)$, the normalized progressive wave streaming stays nearly constant (also for strongly non-linear waves, the contribution of higher harmonics to progressive wave streaming is small). However, the importance of wave shape effect relative to the free surface effect increases, resulting in a reversal from on- to offshore. This $k h$ behaviour is qualitatively consistent with the findings of Trowbridge and Madsen [1984b]. For $A / k_{\mathrm{N}}$ $=320$, the numerical model gives the directional reversal close to $k h=0.8$.

Before, we described that the two physical processes both become stronger when approaching the shore. We learn from Figure 2.8 that velocity-skewness streaming increases the most with decreasing water depth. Apparently, its driving force increases more than the mechanism driving the progressive wave streaming. As discussed in section 2 and confirmed by Figure 2.8, contribution of the latter to $U_{0} / \hat{u}_{1}$ depends on the vertical advection of horizontal momentum that scales with $\hat{u}_{1} / \mathrm{c}$. The increasing relative contribution of wave shape streaming for decreasing water depth can then be explained from the scaling of $\hat{u}_{2} / \hat{u}_{1}$, which is proportional to $\hat{u}_{1} \mathrm{c}^{-1} / \sinh ^{2}(k h)$ when using Stokes' theory. This is confirmed by the simulations for wave shape streaming only that show results proportional to $1 / \sinh ^{2}(k h)$. Figure 2.8 also shows that the streaming from the full model is as good as equal to the sum of separate simulations with wave shape and progressive wave streaming only. This indicates that interaction between the two streaming mechanisms is generally small.
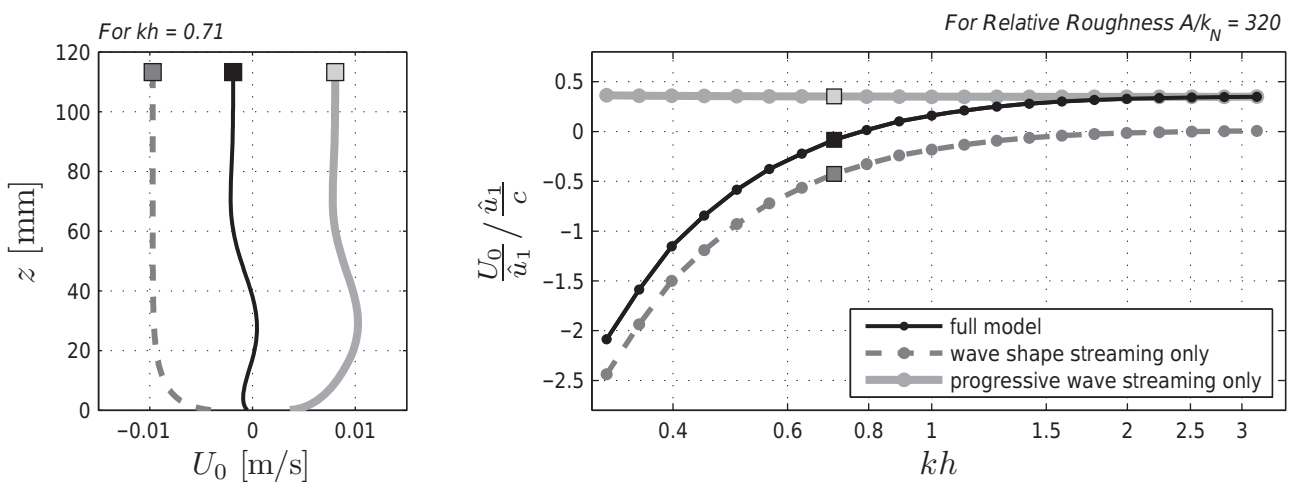

Figure 2.8: Streaming velocities from full model simulations (black), simulations with wave shape effect only (gray / dashed line), and free surface effect only (light gray); Left) streaming profiles for a single $k h$-value; right) non-dimensional streaming at the outer edge of the boundary layer as function of relative water depth $\mathrm{kh}$ (on log-scale) for $A / k_{\mathrm{N}}=320$. 


\subsubsection{Influence of relative bed roughness $\mathbf{A} / \mathrm{k}_{N}$}

Figure 2.9 shows model results for the non-dimensional streaming velocity for various values of $A / k_{\mathrm{N}}$, together with the analytical results of Trowbridge and Madsen [1984b] (TM84). In the numerical results, the main influence of the roughness is that for all $k h$ the streaming value shifts in negative direction for increasing $A / k_{\mathrm{N}}$, with decreasing shifts for larger values of $A / k_{\mathrm{N}}$. The results differ from TM84 in various ways. Firstly, the simulated streaming velocities at $k h=3$, approximating the streaming from progressive wave streaming only, are smaller and much less sensitive for $A / k_{\mathrm{N}}$. Secondly, the numerically predicted $k h$ value of streaming reversal is higher. Finally, at low values of $k h$ the $A / k_{\mathrm{N}}$ influence is opposite in the two models. According to the analytical model results both streaming processes become stronger with increasing bed roughness (decreasing $A / k_{\mathrm{N}}$ ). We conversely found almost no influence of the roughness on the offshore wave shape streaming. Like in validation case 4 , this can be explained by the diffusive transport of t.k.e., which is included in the present model with turbulence memory and $k-\varepsilon$ closure, and not in TM84, with an eddy viscosity being a function of the instantaneous shear velocity.

\subsubsection{Parameterizations}

Parameterizations of the numerical results may be helpful to include progressive wave streaming and wave shape streaming into practical sand transport formulae, that either use a free stream velocity moment (Bagnold-Bailard type) or bed shear stress (Meyer-Peter and Müller type) as hydrodynamic input (e.g. Van Rijn [2007], Nielsen [2006]). The results for the streaming at the top of a rough

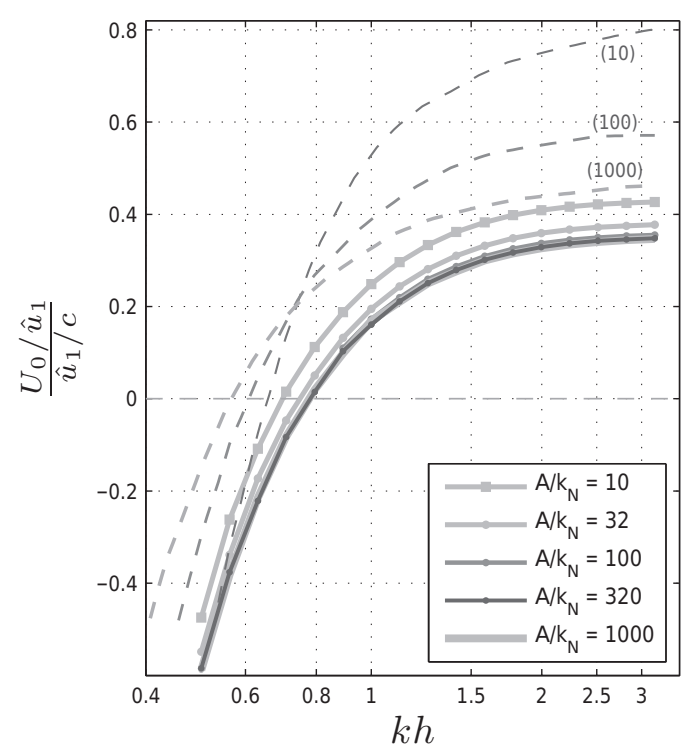

Figure 2.9: Non-dimensional streaming velocity at the outer edge of the boundary layer as function of $k h$ (log-scale) for various values of the relative roughness parameter $A / k_{\mathrm{N}}$. Solid lines: present model results. Dashed lines: analytical results of Trowbridge and Madsen [1984b]. 
turbulent boundary layer can be parameterized as follows:

$$
\frac{U_{0} / \hat{u}_{1}}{\hat{u}_{1} / c}=0.345+0.7\left(\frac{A}{k_{N}}\right)^{-0.9}-\frac{0.25}{\sinh ^{2}(k h)}
$$

(with the first two terms parameterizing progressive wave streaming and the last term connected to wave shape streaming beneath Stokes waves).

The current related mean bed shear stress and the contributions to it from the wave Reynolds stress and the wave shape effect (see equation (2.19)), have been studied just like the streaming velocities. When we normalize the contributions at the bed by $\tau_{\mathrm{b}, \mathrm{m}} \hat{u}_{1} / c$, the results shows a $k h$-dependency similar to Figure 2.8, but now independent of $A / k_{\mathrm{N}}$. Without a mean pressure gradient, the total mean bed shear stress is equal to the wave Reynolds stress $\bar{\tau}_{b, W R S}=-\rho \overline{u w}_{\infty}$. We found from the numerical simulations:

$$
\bar{\tau}_{b}=\rho\left[\left(v+\bar{v}_{t}\right) \frac{\partial \bar{u}}{\partial z}+\overline{\tilde{v}_{t} \frac{\partial \tilde{u}}{\partial z}}\right]=\bar{\tau}_{b, W R S}=0.430 \tau_{b, m} \hat{u}_{1} c^{-1}
$$

With $\tau_{b, m}=1 / 2 \rho f_{w} \hat{u}_{1}{ }^{2}$, this gives:

$$
\bar{\tau}_{b, W R S}=0.215 \rho f_{w} \hat{u}_{1}^{3} c^{-1}
$$

which numerically confirms earlier analytical estimates for $-\rho \overline{u w}_{\infty}$ from energy dissipation $D_{\mathrm{E}}$ in a sinusoidal oscillation:

$$
-\rho \overline{u w}_{\infty}=D_{E} / c \quad D_{E}=\overline{\vec{\tau}_{b}(t) \tilde{u}_{\infty}(t)}=\frac{2}{3 \pi} \rho f_{w} \hat{u}_{1}^{3}
$$

as applied before [Nielsen, 2006] to include progressive wave streaming in practical sand transport formulae.

\subsubsection{Effects of a mean pressure gradient on current and stress}

In reality, the boundary layer may also be affected by a mean pressure gradient, related to return current, undertow or effects of wave transformation on a sloping beach. This mean pressure gradient is not included in the simulations (and parameterizations) of section 2.4.2. We explore the influence of a mean pressure gradient on the mean current and stress components with the numerical model. Based on CASE 4: Van Doorn, with a mean pressure gradient of $0.2 \mathrm{~Pa} / \mathrm{m}$, we define three additional cases: with respectively a strongly increased positive mean pressure gradient, a zero mean, and a strong negative mean pressure gradient. The results are shown in Figure 2.10. Panel (e) for $U_{0}$ shows that the mean pressure gradients have large effects on both magnitude and shape of the $U_{0}$ profile inside the wave boundary layer. Not only the extreme cases, but also the simulation with zero mean pressure gradient show significant differences with the validation case. Panel (a) to (d) show the 
current related shear stress and the various contributions to it, see equation (2.19). The wave shape contribution (d) decreases with increasing pressure gradient, which is according expectation: a negative mean current reduces the difference between on- and offshore turbulence beneath the velocity-skewed wave. The contribution of the pressure gradient (c) is substantial: in the original validation case 4 , with only a small return current, the contribution from the pressure gradient at the bed is already $1 / 3$ of the wave Reynolds stress (b) at the bed. We can also observe that the wave Reynolds stress (b) at the bed is not affected by an adapted mean pressure gradient. So also with strong undertow or shoaling effects, the wave Reynolds stress contribution to the mean bed shear stress can be modeled with equation (2.25).

Estimates of realistic mean pressure gradients, that not only depend on the local situation, might be obtained from wave properties, mass-fluxes and geometric information through undertow models. See also Zhang et al., [2011] who studied the wave boundary layer beneath shoaling and breaking waves, both generating mean pressure gradients, with a first order boundary layer model. The coupling to undertow models has not been tested here.

(a)

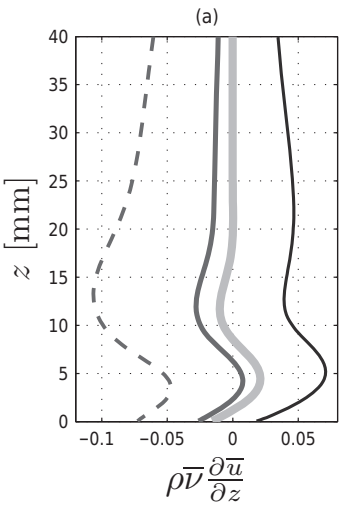

(b)

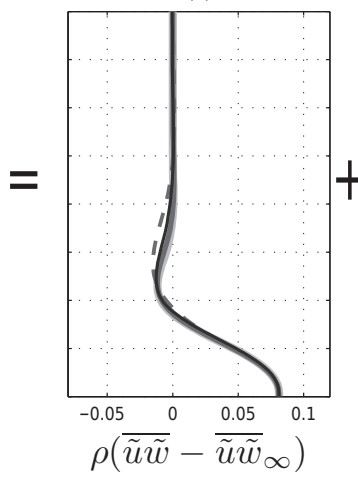

(c)

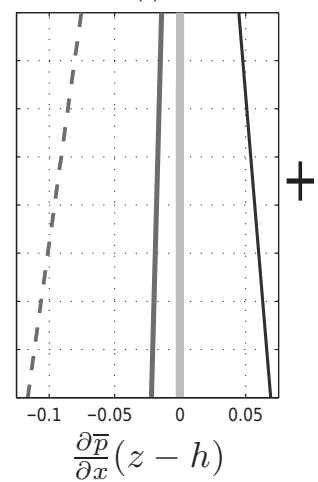

(e)

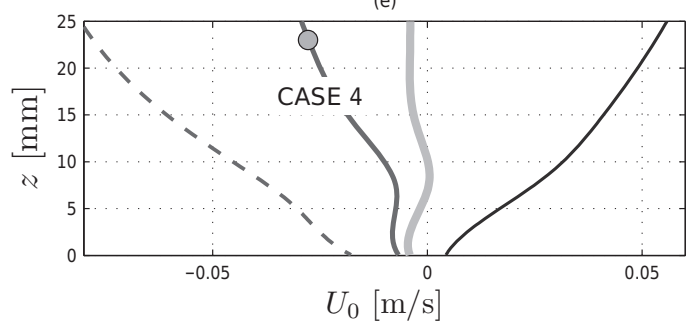

(d)

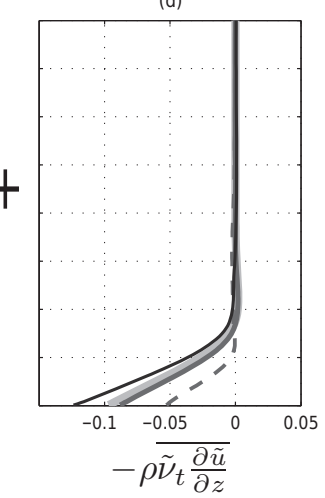

$-\rho \overline{\tilde{\nu}_{t} \frac{\partial \tilde{u}}{\partial z}}$ 


\subsection{DISCUSSION}

The main motive for this hydrodynamic study on wave boundary layer streaming is its potential influence on total sediment transport and nearshore morphology. Progressive wave streaming might explain the differences found in sand transport between tunnel and flume experiments. This is especially relevant, because most morphodynamic models use shear stress and transport formulations primarily based on tunnel experiments, and also tend to under predict onshore transport in accreting conditions [Van Rijn et al., 2011]. To show the potential importance of progressive wave streaming for sediment transport, we apply the numerical model both with (BL2-version) and without free surface effects (BL1-version) for two conditions of the full scale flume experiments of Dohmen-Janssen and Hanes [2002] (MI and MH, both with grain size $d_{50}=0.24 \mathrm{~mm}$ ). Following the example of Gonzalez Rodriquez and Madsen [2011], we use the simulated time-dependent bed shear stress $\tau_{\mathrm{b}}(\mathrm{t})$ from both versions as input to a bed load sediment transport formula [Nielsen, 2006]:

$$
\frac{q_{s}(t)}{\sqrt{\left(\rho_{s} / \rho_{w}-1\right) g d_{50}^{3}}}=\left\{\begin{array}{cc}
12[|\theta(t)|-0.05] \sqrt{|\theta(t)|} \frac{\theta(t)}{|\theta(t)|} & \text { for }|\theta(t)|>0.05 \\
0 & \text { for }|\theta(t)| \leq 0.05
\end{array}\right\}
$$

where $q_{\mathrm{s}}(\mathrm{t})$ and $\theta(\mathrm{t})$ are the time dependent sediment transport and Shields parameter respectively. The latter is computed from the model results for $\tau_{\mathrm{b}}(\mathrm{t})$ through:

$$
\theta(t)=\frac{\tau_{b}(t)}{\left(\rho_{s}-\rho_{w}\right) g d_{50}}
$$

To account for the higher roughness of the mobile bed, a bed roughness height $k_{\mathrm{N}}$ (model input) is used of the order of the maximum sheet-flow layer thickness in these experiments $\left(k_{\mathrm{N}}=20 D_{50}\right)$. Figure 2.11 shows results for $\tau_{\mathrm{b}}(\mathrm{t})$ and net transport rate $\left\langle q_{\mathrm{s}}>\right.$ from BL1 and BL2. The predicted $\left\langle q_{\mathrm{s}}\right\rangle$ increases with $40 \%$ in case $\mathrm{MH}$ and even $100 \%$ in case MI. So in the latter case, the contribution of progressive wave streaming to onshore transport is of the same order of magnitude as the contribution of velocity-skewness. In both cases, the measured $\left\langle q_{\mathrm{s}}\right\rangle$ is approached the best with progressive wave streaming included. Note that the numerical framework of the present model, shown to have some important advantages over the analytical approach concerning the hydrodynamics (see 2.3.4), also allows to investigate the role of streaming for fine sands, with much more sand in suspension. The question whether streaming is the full explanation of the differences in transport found in tunnel and flume will be discussed both for medium and fine sized sands in a future article, including a systematic data-model comparison involving all available large scale flume data.

Although the test cases 1 to 4 are represented by the model reasonably well, they still show sometimes small differences between the measured and computed mean and unsteady flow near the bed. The question could therefore be raised whether these inaccuracies may form a serious shortcoming of the model when applied to sediment transport predictions. What is the deviation in predicted sediment transport these errors might introduce and how does this compare to the effects 
of progressive wave streaming we pointed at before? To get an impression hereof, we study the influence of inaccuracies in mean and unsteady flow on the third-order velocity moment $\left\langle u^{3}\right\rangle$. We do this for CASE 4 , for which near the bed $(0-5 \mathrm{~mm})$ the negative streaming was somewhat overpredicted and the phases of the harmonic components were underpredicted, the latter explained by the model's underestimation of the friction. We study $\left\langle u^{3}\right\rangle$ because in this region very close to the bed, it is reasonable to assume that $\tau_{\mathrm{b}}(\mathrm{t}) \sim|u(t)| u(t)$ and $q_{\mathrm{s}}(\mathrm{t}) \sim \tau_{\mathrm{b}}(t) u(t) \sim u(t)^{3} \quad$ (at least for medium sized sand, neglecting phase-lags of suspended sediment), see Bailard [1981], Ribberink and Al-Salem [1994]. Figure 2.12 shows $\left\langle u^{3}\right\rangle$ computed from the experimental data and as computed by the model (BL2). Next, $\left\langle u^{3}\right\rangle$ has also been computed from a simulation without progressive wave streaming (BL1), and also again from the BL2 model but now with the computed mean velocity near the bed $(0-10 \mathrm{~mm})$ replaced by an approximation of the measured mean current $(-0.0025 \mathrm{~m} / \mathrm{s})$. In this way, possible differences between the first and the last computation can only be caused by inaccuracies in the simulated unsteady velocities. Figure 2.12 shows that the influence of unsteady flow inaccuracies on $\left\langle u^{3}\right\rangle$ is very small compared to steady flow inaccuracies, and the latter are much smaller than deviations introduced by neglecting progressive wave streaming (BL1). This underlines the primary importance of a good streaming prediction for sediment transport prediction in this case. At the same time, the present model performance in prediction of the nearbed unsteady flow seems to be sufficient.
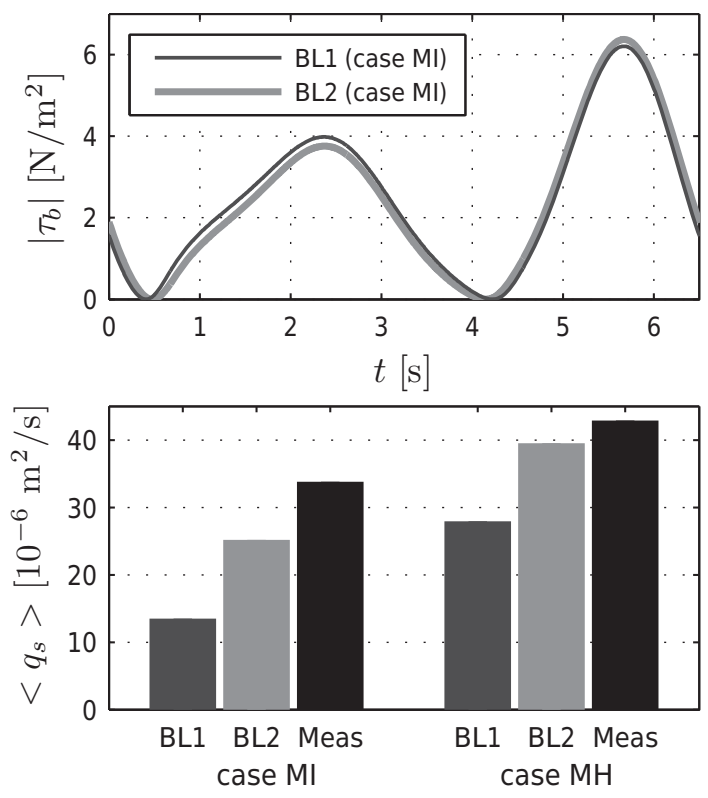

Figure 2.11: Top) Absolute bed shear stresses $\left|\tau_{\mathrm{b}}\right|$ as function of time for case MI of Dohmen-Janssen and Hanes [2002] computed both without (BL1) and with (BL2) progressive wave streaming; Bottom) Net sand transport rates $\left\langle q_{\mathrm{s}}>\right.$ for case MI and MH of Dohmen-Janssen and Hanes [2002] as determined from $\tau_{\mathrm{b}}(\mathrm{t})$ both without and with progressive wave streaming through a bed load transport formulae, compared with measured transport rates (Meas). 
Concerning the validity of the model assumptions it should be noted that the results in section 2.4.2 have been obtained using Stokes theory to determine the wave shape. Seaward of the surfzone, where waves are predominantly velocity skewed with limited non-linearity and accelerationskewness is nearly absent [Ruessink et al., 2009], this approximation is valid and the presented results can be applied. Note that the model itself is very well able to deal with the effects of larger non-linearity and acceleration-skewness on the boundary layer, as shown in section 3. So with a more advanced predictor of the wave shape, the model can also be applied in more shallow water and the surf zone. However, note that there also turbulence effects of (especially plunging) wave breakers may start to effect the boundary layer flow ([Fredsøe et al., 2003], [Scott et al., 2009]).

Finally, preliminary simulations with the present model including sediment and buoyancy-effects show a slight influence of suspended sediment on streaming, especially for fine sediment, most likely related to turbulence damping by density stratification. This asks for re-validation of the model on measured velocities above mobile beds when the contribution of progressive wave streaming to transport rates will be studied in more detail. Also here, reference is made to a future article which is focused on sediment transport prediction with the BL1 and BL2 models.

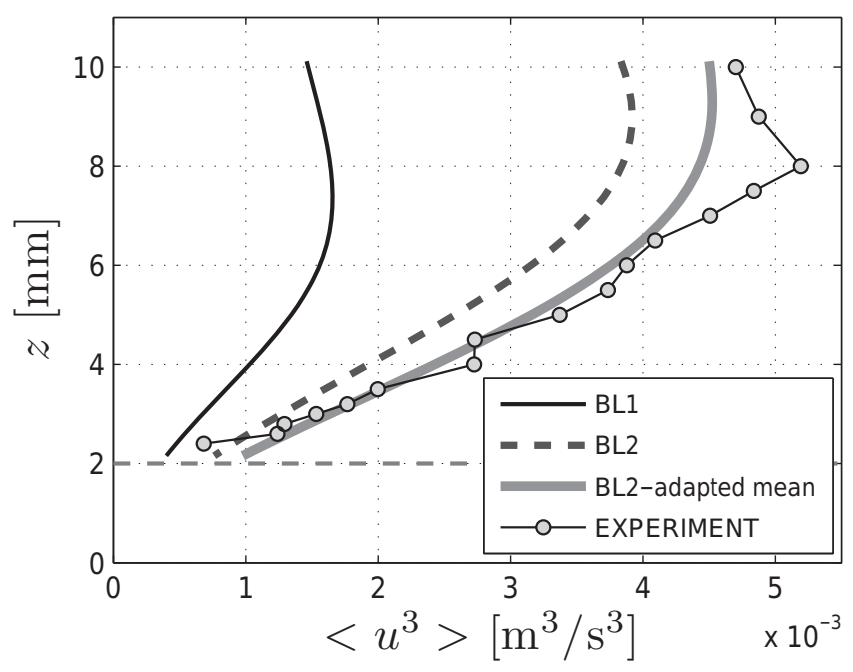

Figure 2.12: Third order velocity moment $\left\langle u^{3}\right\rangle$ as function of $z$, computed from the EXPERIMENT of CASE 4: Van Doorn; from results of simulations without (BL1) and with (BL2) progressive wave streaming; and from the BL2 model results with the computed mean velocity replaced by an approximation of the measured mean $(-0.0025 \mathrm{~m} / \mathrm{s})$. 


\subsection{CONCLUSION}

A numerical boundary layer model has been developed to investigate the net current and shear stress in the bottom boundary layer as determined by wave shape effects and free surface effects. The latter have been taken into account by inclusion of advection of momentum and turbulence properties into the 1DV-RANS model formulations and $k-\varepsilon$ turbulence closure.

The model has been validated with good agreement on a selection of experimental cases with different types of wave boundary layer flow. This fills a gap in literature on comparison of numerical models with measured mean wave boundary layer currents. The validation showed that both streaming processes, wave shape streaming and progressive wave streaming, need to be considered to reproduce the measurements. Besides, the turbulence memory in the model's $(\mathrm{k}-\varepsilon)$ turbulence closure and the presence of more harmonic velocity components contributes significantly to improved reproductions compared to earlier analytical modeling of streaming, e.g. the accurate reproduction of observed offshore current beneath acceleration-skewed waves where earlier analytical models failed.

Subsequently, the model has been used to investigate the changing balance between offshore wave shape streaming and onshore progressive wave streaming for varying wave and bed conditions (section 2.4.2), by studying their contribution to the non-dimensional streaming velocity $U_{0} c / \hat{u}_{1}^{2}$ in the parameter space spanned by relative water depth $k h$ and roughness parameter $A / k_{\mathrm{N}}$. At relative deep water (large $k h$ ) the streaming is completely determined by the free surface effect. For decreasing relative water depth $(k h)$, the normalized progressive wave streaming stays nearly constant, but the importance of wave shape effect relative to the free surface effect increases. The effect of bed roughness is less distinct. For increasing relative bed roughness (decreasing $A / k_{\mathrm{N}}$ ), we found slightly stronger onshore progressive wave streaming. These model results have been parameterized in an expression for the streaming velocities at the top of the boundary layer as function of $k h$ and $A / k_{\mathrm{N}}$, see equation (6.1). The model results for the contribution of progressive wave streaming to the normalized mean bed shear stress do not show a roughness dependency and give a numerical confirmation of earlier analytical estimates hereof for sinusoidal waves, which are shown to apply also when a strong pressure gradient is present (section 2.4.3).

Other insights obtained during this study are that the maximum offshore current resulting from velocity-skewness takes place on a lower level in the bottom boundary layer than the maximum onshore current from the progressive wave streaming. Therefore, layers with positive and negative shear $\left(\partial U_{0} / \partial z\right)$ can generally be observed in the mean current profile when both mechanisms are active. Next, the effect from acceleration-skewness is basically the same as the effect from velocityskewness: a difference in turbulence properties during on- and offshore movement results in an offshore mean current. However, the acceleration-skewness effect is smaller and the level of the maximum offshore current is closer to the bed. 
An exploration of the potential importance of the model results for sediment transport modeling is given in section 2.5, showing that increased bed shear stress due to progressive wave streaming leads to larger predicted sediment transport under waves, better matching the data. It is finally concluded that the validated numerical model provides a modeling framework for follow-up research on the question whether progressive wave streaming is the full explanation of the different sediment transport rates found in tunnel and flume experiments. 


\title{
3 SAND TRANSPORT BENEATH WAVES: THE ROLE OF PROGRESSIVE WAVE STREAMING AND OTHER FREE SURFACE EFFECTS ${ }^{2}$
}

\begin{abstract}
Recent large scale wave flume experiments on sheet-flow sediment transport beneath Stokes waves show more onshore directed sediment transport than earlier sheet-flow experiments in oscillating flow tunnels. For fine sand this extends to a reversal from offshore (tunnels) to onshore (flumes) directed transport. A remarkable hydrodynamic mechanism present in flumes (with free water surface) but not in tunnels (rigid lid) is the generation of progressive wave streaming, an onshore wave boundary layer current. This paper investigates whether this streaming is the full explanation of the observed differences in transport. Hereto we present a numerical model of wave-induced sand transport that includes the effects of the free surface on the bottom boundary layer. With these effects and turbulence damping by sediment included, our model yields good reproductions of the vertical profile of the horizontal (mean) velocities, as well as transport rates of both fine and medium sized sediment. Similar to the measurements, the model reveals the reversal of transport direction by free surface effects for fine sand. A numerical investigation of the relative importance of the various free surface effects shows that progressive wave streaming indeed contributes substantially to increased onshore transport rates. However, especially for fine sands, horizontal gradients in sediment advection in the horizontally non-uniform flow field also are found to contribute significantly. We therefore conclude that not only streaming, but also inhomogeneous sediment advection should be considered in formulas of wave-induced sediment transport applied in morphodynamic modeling. We propose a variable time-scale parameter to account for these effects.
\end{abstract}

\footnotetext{
${ }^{2}$ This chapter has been accepted for publication as: Kranenburg, W.M., J.S. Ribberink, J.L.M. Schretlen and R.E. Uittenbogaard (2013), Sand transport beneath waves: the role of progressive wave streaming and other free surface effects, Journal of Geophysical Research. DOI:10.1029/2012JF002427.
} 


\subsection{INTRODUCTION}

The development of cross-shore and long-shore coastal bottom profiles is strongly determined by the dynamics of water and sediment in the bottom boundary layer induced by surface waves. This has been the rationale for many experimental, analytical and numerical studies on the interaction between wave motion and sand beds. Understanding of the interaction processes steers the development of parameterized sediment transport formulas that are feasible in large-scale morphodynamic simulations. Finally, these large-scale simulations provide insight into coastal bottom profile developments.

A research topic of many wave-bed interaction studies is the influence of the wave shape on flow velocities, bed shear stresses and sediment transport rates. These studies either focus on velocity skewness (present under waves with amplified crests), acceleration skewness (present under waves with steep fronts) or both phenomena in joint occurrence (for references see Ruessink et al. [2009]). The experimental studies on wave shape effects have been carried out in oscillating flow tunnels (with horizontally uniform flow), with both fixed and mobile flat beds of various sand grain sizes, and with special attention paid to the sheet-flow transport regime, where bed forms are washed away and the bed is turned into a moving sediment layer [Ribberink et al., 2008]. An important observation from tunnel experiments in the sheet-flow regime is that under velocity-skewed flow over coarse grains the sediment transport is mainly onshore, but that net transport decreases with decreasing grain sizes and can even become negative [O'Donoghue and Wright, 2004]. An explanation for this is the phase-lag effect: rather fine sediment is stirred up by the strong onshore motion, settles only slowly, is still partly suspended during flow reversal and is subsequently transported offshore [Dohmen-Janssen et al., 2002]. Studies on the effect of acceleration skewness (e.g. [Van der A et al., 2011]) have revealed that the increased acceleration during the onshore motion results in increased near bed vertical velocity gradients and bed shear stresses. This enhances sediment pick-up and net onshore transport. For purely acceleration-skewed oscillations over fine sand, the phase-lag effect also contributes to onshore transport: more time is available for settling subsequent to maximum onshore flow and less following maximum offshore flow.

Dohmen-Janssen and Hanes [2002] and very recently Schretlen [2012] carried out detailed experiments on sand transport under velocity-skewed waves over flat beds in full-scale wave flumes. The flume experiments of Dohmen-Janssen and Hanes [2002] show larger transport rates for medium grain sizes compared to tunnel experiments with similar velocity skewness. Schretlen [2012] even found a reversed transport direction for fine sands in flumes (onshore) compared to tunnels (offshore). An explanation of the increased onshore transport brought up in these studies is 'progressive wave streaming', an onshore directed bottom boundary layer current under influence of vertical orbital motions in the horizontally non-uniform flow beneath progressive waves [LonguetHiggins, 1953]: the vicinity of the bed affects the phase difference between the horizontal and vertical orbital velocities. This introduces a wave-averaged transport of horizontal momentum towards the bed that drives the onshore current. Note that this process acts opposite to the net current 
generated in a turbulent bottom boundary layer by a velocity-skewed or acceleration-skewed oscillation ('wave shape streaming'). The latter mechanism is due to wave shape induced differences in time-dependent turbulence during the on- and offshore phases of the wave, which causes a nonzero wave-averaged turbulent shear stress ([Trowbridge and Madsen, 1984], [Ribberink and AlSalem, 1995], [Fuhrman et al., 2009]). We studied the streaming and the changing balance between the generation mechanisms for varying wave conditions above fixed beds in Kranenburg et al. [2012]. In this study we investigate numerically to what extent progressive wave streaming can explain the differences in transport of both medium and fine sized sand between tunnel and flume experiments. Further questions are: what other processes are introduced by the progressive character of the free surface wave and how do they influence sand transport for various grain sizes? A good understanding of the tunnel-flume differences is relevant, because many transport formulas used in morphodynamic computations in science and engineering are based on tunnel experiments and do not include the flume and prototype free surface effects. This study should therefore contribute to improvement of these formulas.

Free surface effects have been included in earlier modeling studies. For example, Gonzalez Rodriquez [2009] predicted the contribution of progressive wave streaming to onshore transport by coupling a higher order analytical boundary layer model with a bed load transport formula. However, this concept cannot be applied to fine sand. Henderson et al. [2004] and Hsu et al. [2006] studied sand bar migration with a clear fluid (single phase) fixed bed numerical boundary layer model with advection-diffusion formulation for suspended sediment concentrations. A similar model was used by Holmedal and Myrhaug [2009] and Blondeaux et al. [2012], both of which found significant differences in transport rates between tunnel- and sea wave simulations. Although their results are qualitatively consistent with the experimental data, no specification of the progressive wave streaming contribution hereto or quantitative comparison with flume measurements was provided in these studies. Also, the single phase studies mentioned above do not consider the details of the sediment pick-up and the effects of high sediment concentrations on grain settling velocity and turbulence. However, sediment-induced turbulence damping can largely affect velocity profiles and transport rates, especially for fine sediment, see e.g. Winterwerp [2001] (for steady flow) and Conley et al. [2008] and Hassan and Ribberink [2010] (oscillatory flow). Yu et al., [2010] studied progressive wave effects with a two-phase model that explicitly accounts for fluid-grain and graingrain interactions within the sheet-flow layer. However, until now this model-type has only been validated for large to medium grain sizes ( $>0.2 \mathrm{~mm})$ [Amoudry et al., 2008].

Compared to the single phase modeling studies above, the present study has three innovative aspects. Firstly, we use a model that includes both free surface effects and sediment related reduction of turbulence and settling velocities. Secondly, we present an extensive quantitative model validation on boundary layer flow beneath full scale waves over a mobile bed, as well as on net transport of both fine and medium sediment in both tunnel and flume experiments. This detailed validation could only be carried out because detailed full scale flume measurements became available recently [Schretlen, 2012]. A third new aspect is the differentiation between transport 
related to progressive wave streaming and related to other free surface effects, which we use to develop parameterizations for practical transport formulas.

The outline of this paper is as follows: section 2 describes our numerical model. The data used for model validation and the validation itself are described in section 3. Section 4 describes the model experiments quantifying the contribution of various free surface effects. The results are discussed in section 5, with a focus on their relevance for sediment transport formulas used in morphodynamic modeling. Our major conclusions are summarized in Section 6.

\subsection{MODEL FORMULATION}

Our model can be classified as a 1DV Reynolds Averaged Navier-Stokes flat bed boundary layer model with $k-\varepsilon$ closure for turbulence and an advection-diffusion formulation for suspended sediment. It is an extension of the hydrodynamic model described in Kranenburg et al. [2012] with a sediment balance and feedback of sediment on the flow. The sediment formulations correspond to those in the previous model version used by Ruessink et al. [2009], originally developed by Uittenbogaard et al. [2001], now extended with advective terms. The main differences with Henderson et al. [2004], Holmedal and Myrhaug [2009] and Blondeaux et al. [2012] appear in the turbulence formulations (stratification effects) and, in the latter two cases, in the forcing of the model.

\subsubsection{Basic Equations}

The fundamental unknowns solved by the model are horizontal flow velocity $u$, vertical flow velocity $w$, sediment concentration $c$ and turbulent kinetic energy $k$ and its rate of dissipation $\varepsilon$. The flow velocities are solved from the following equations:

$$
\begin{gathered}
\frac{\partial u}{\partial t}+u \frac{\partial u}{\partial x}+w \frac{\partial u}{\partial z}=-\frac{1}{\rho_{w}} \frac{\partial p}{\partial x}+\frac{\partial}{\partial z}\left\{\left(v+v_{t}\right) \frac{\partial u}{\partial z}\right\} \\
\frac{\partial u}{\partial x}+\frac{\partial w}{\partial z}=0
\end{gathered}
$$

where $p$ the pressure, $\rho_{\mathrm{w}}$ the fluid density, $v$ the kinematic viscosity of water, $v_{\mathrm{t}}$ the turbulence viscosity, $x$ and $z$ the horizontal and vertical coordinate, positive in onshore and upward direction respectively.

The closure for $v_{\mathrm{t}}$ is provided by a $k-\varepsilon$ model $[R o d i, 1984]$, where $k$ is the turbulent kinetic energy, $\varepsilon$ the energy dissipation rate and their relation to $v_{\mathrm{t}}$ :

$$
v_{t}=c_{\mu} \frac{k^{2}}{\varepsilon}
$$

The turbulence quantities are solved from the following equations: 


$$
\begin{gathered}
\frac{\partial k}{\partial t}+u \frac{\partial k}{\partial x}+w \frac{\partial k}{\partial z}=\frac{\partial}{\partial z}\left\{\left(v+\frac{v_{t}}{\sigma_{k}}\right) \frac{\partial k}{\partial z}\right\}+P_{k}-\varepsilon-B_{k} \\
\frac{\partial \varepsilon}{\partial t}+u \frac{\partial \varepsilon}{\partial x}+w \frac{\partial \varepsilon}{\partial z}=\frac{\partial}{\partial z}\left\{\left(v+\frac{v_{t}}{\sigma_{\varepsilon}}\right) \frac{\partial \varepsilon}{\partial z}\right\}+\frac{\varepsilon}{k}\left(c_{1 \varepsilon} P_{k}-c_{2 \varepsilon} \varepsilon-c_{3 \varepsilon} B_{k}\right)
\end{gathered}
$$

where $P_{\mathrm{k}}$ is the turbulence production and $B_{\mathrm{k}}$ the buoyancy flux. $\sigma_{\mathrm{k},}, \sigma_{\varepsilon,} c_{\mu}, \mathrm{c}_{1 \varepsilon}$ and $c_{2 \varepsilon}$ are constants. We apply $\left(\sigma_{\mathrm{k}}, \sigma_{\varepsilon,}, c_{\mu}, c_{1 \varepsilon,}, c_{2 \varepsilon}\right)=(1.0,1.3,0.09,1.44,1.92)$ (standard values, Rodi [1984]). The production term $P_{\mathrm{k}}$ yields:

$$
P_{k}=v_{t}\left(\frac{\partial u}{\partial z}\right)^{2}
$$

The buoyancy flux $B_{\mathrm{k}}$ accounts for the conversion of turbulent kinetic energy to mean potential energy (or vice versa) with the mixing of sediment, treated equivalent to buoyancy flux in a saltstratified or thermally-stratified flow. In a stable stratification $(\partial \rho / \partial z<0)$, this flux will lead to turbulence reduction, in case of an unstable stratification to turbulence generation. Besides, in the latter case the upward jets (by Rayleigh-Taylor instabilities) from the lighter fluid into the denser fluid on top of it produce extra vorticity, which is, considering the parallel between vorticity and $\varepsilon$ (TKE-dissipation), accounted for by an increase of $\varepsilon$. This is described with the following expressions for the buoyancy flux $B_{\mathrm{k}}$, the Brunt-Väisälä frequency $N$ and $c_{3 \varepsilon}$ :

$$
B_{k}=\frac{v_{t}}{\sigma_{p}} N^{2} \quad ; \quad N^{2}=-\frac{g}{\rho_{m}} \frac{\partial \rho_{m}}{\partial z} \quad ; \quad c_{3 \varepsilon}= \begin{cases}0 & N^{2} \geq 0 \\ 1 & N^{2}<0\end{cases}
$$

where $\sigma_{\mathrm{p}}$ is a constant, in this case equal to the turbulence Prandtl-Schmidt number $\sigma_{\mathrm{t}}$ for conversion of turbulence viscosity $v_{\mathrm{t}}$ into eddy diffusivity of sediment, $g$ the gravitational acceleration and $\rho_{\mathrm{m}}$ the density of the local water-sediment mixture $\rho_{\mathrm{m}}=\rho_{\mathrm{w}}+\left(\rho_{\mathrm{s}}-\rho_{\mathrm{w}}\right) c$.

The sediment (volume) concentration $c$ is solved from a sediment balance:

$$
\frac{\partial c}{\partial t}+u \frac{\partial c}{\partial x}+w \frac{\partial c}{\partial z}=\frac{\partial w_{s} c}{\partial z}+\frac{\partial}{\partial z}\left\{\left(v+\frac{v_{t}}{\sigma_{t}}\right) \frac{\partial c}{\partial z}\right\}
$$

where we apply $\sigma_{\mathrm{t}}=0.7$ (as derived from experiments by Breugem [2012]). The local sediment fall velocity $w_{\mathrm{s}}$ is determined using the undisturbed settling velocity $w_{\mathrm{s}, 0}$ according Van Rijn [1993], with a correction for hindered settling in high sediment concentrations following Richardson and Zaki [1954]:

$$
w_{s}=w_{s, 0}\left(1-\frac{c}{c_{s}}\right)^{p} ; \quad w_{s, 0}=\frac{10 v}{d_{50}}\left[\left(1+\frac{0.01 \Delta g d_{50}^{3}}{v^{2}}\right)^{1 / 2}-1\right] \quad \text { for } 0.1 \mathrm{~mm}<d_{50}<1.0 \mathrm{~mm}
$$


with $c_{\mathrm{s}}=0.65, p=5$ and $\Delta=\left(\rho_{s}-\rho_{w}\right) / \rho_{w}$.

Assuming uniformity of wave shape and height during propagating over the horizontal sand bed, the model is reduced to a $1 \mathrm{DV}$-model by transformation of horizontal gradients of velocity, turbulence properties and sediment concentration into time derivatives, using:

$$
\frac{\partial \ldots}{\partial x}=-\frac{1}{c_{p}} \frac{\partial \ldots}{\partial t}
$$

where $c_{\mathrm{p}}$ is the wave propagation speed.

The consideration of advective transport of horizontal momentum, turbulence properties and sediment marks the fundamental difference between modeling the horizontally uniform situation like in oscillating flow tunnels or the horizontally non-uniform situation beneath progressive surface waves in prototype situation and wave flumes. The progressive wave streaming is driven by the wave averaged vertical advective transport of horizontal momentum into the wave boundary layer (wave Reynolds stress).

\subsubsection{Forcing}

The model can be forced in two ways. In the 'match model' formulation, the principally unknown $u(t, z)$ is forced to match a predefined horizontal velocity signal at a certain vertical level, e.g. a measured time-series. The associated (oscillating plus mean) pressure gradient is determined iteratively every time step from equation (3.1) at the matching level. In the alternative 'free model' formulation, the oscillating horizontal pressure gradient is determined in advance from a given free stream horizontal velocity $\tilde{u}_{\infty}$ (or $u_{\text {red }}$ ) with zero mean, using:

$$
-\frac{1}{\rho} \frac{\partial \tilde{p}}{\partial x}=\frac{\partial \tilde{u}_{\infty}}{\partial t}+\tilde{u}_{\infty} \frac{\partial \tilde{u}_{\infty}}{\partial x}
$$

In the latter approach mass transport arising from streaming mechanisms and Stokes' drift is not compensated by a return flow driven by an additional mean pressure gradient and the mean current is allowed to develop freely. This formulation needs a predefined oscillating free stream velocity as input.

\subsubsection{Boundary conditions}

To solve equation (3.1), (3.4) and (3.5), we apply the upper boundary conditions:

$$
\left.v_{t} \frac{\partial u}{\partial z}\right|_{z=t o p}=0 \quad ;\left.\quad \frac{\partial k}{\partial z}\right|_{z=t o p}=0 \quad ;\left.\quad \frac{\partial \varepsilon}{\partial z}\right|_{z=t o p}=0
$$

and the lower boundary conditions: 


$$
\left.\frac{\partial u}{\partial z}\right|_{z=0}=\frac{u_{*}}{9 \kappa z_{0}} \quad ;\left.\quad k\right|_{z=0}=\frac{u_{*}{ }^{2}}{\sqrt{c_{\mu}}} \quad ;\left.\quad \varepsilon\right|_{z=0}=\frac{u_{*}^{3}}{9 \kappa z_{0}}
$$

Here $u_{*}$ is the friction velocity, $\kappa=0.41$ is the Von Karman constant, and $z_{0}$ is the roughness height. The lower boundary conditions assume hydraulically rough turbulent flow near the bed and are applied at a fixed bottom level. We relate $z_{0}$ to the median sand grain size $d_{50}$ by applying Nikuradse roughness height $k_{\mathrm{N}}=2 d_{50}$ and $z_{0}=k_{\mathrm{N}} / 30$.

The sediment balance of equation (3.8) is solved using a no-flux condition at the top boundary and a pick-up function at reference height $z=z_{\mathrm{a}}=2 d_{50}$. The latter reads:

$$
w_{s} c_{b}+\left.\left(v+\frac{v_{t}}{\sigma_{t}}\right) \frac{\partial c}{\partial z}\right|_{z=z_{a}}=0
$$

For the reference concentration $c_{\mathrm{b}}$ we use the expression of Zyserman and Fredsoe [1994]:

$$
c_{b}(t)=\frac{0.331\left(\theta-\theta_{c}\right)^{1.75}}{1+\frac{0.331}{C_{m}}\left(\theta-\theta_{c}\right)^{1.75}}
$$

a function of the instantaneous Shields parameter $\theta$, the critical Shields parameter $\theta_{\mathrm{c}}$ for initiation of motion [Van Rijn, 1993] and a constant $C_{\mathrm{m}}$, set to 0.32 for oscillatory flow [Zyserman and Fredsoe, 1994]. This reference concentration expression is an empirical relation originally based on near-bed concentration measurements in steady flow and the assumption of Rouse concentration profiles for suspended sediment. In the thin layer beneath $z=z_{\mathrm{a}}$, we apply $c(z)=\left.c\right|_{z=z_{a}}$.

\subsection{VALIDATION}

The validation of the model consists of four parts. We firstly investigate the quality of the model in reproducing boundary layer flow above a mobile bed (0). Because of our interest in the role of streaming in explaining the different trends in observed sediment transport rates in flumes and tunnels, we focus hereby especially on the mean current. Subsequently, we compare model and data for net sediment transport rates (3.3.3). A separate section is dedicated to the model reproduction of the observed different trends in transport as function of velocity moments (3.3.4). Finally, we conclude the validation with a sensitivity analysis and discussion (3.3.5). This section starts with a description of the experimental data used in the model validation (3.3.1).

\subsubsection{Experimental data for model validation}

The model-data comparison on flow velocities is carried out with data from the full scale wave flume experiments described by Schretlen et al. [2011] and Schretlen [2012]. In these recent experiments, regular trochoidal waves of varying wave period $T$ and wave height $H$ were sent 
through a $280 \mathrm{~m}$ long wave flume with water of $3.5 \mathrm{~m}$ depth above a horizontal sand bed with a median grain size $d_{50}$ of $0.245 \mathrm{~mm}$ and $0.138 \mathrm{~mm}$ respectively. At the end, the waves were absorbed by a dissipative beach. Multiple experimental runs (both 30 and 60 minutes runs) were carried out for each wave condition. At $110 \mathrm{~m}$ from the wave generator a frame with various instruments was fixed to the flume wall: among them an Ultrasonic Velocity Profiler (UVP) which was used to obtain detailed vertical profile measurements of the velocity inside the wave boundary layer. This makes these experiments the first that offer detailed information on the boundary layer flow beneath full scale waves over a mobile, flat bed. Before and after each run, the horizontal profile of the bed was measured either with a rolling bed profiler or with echo sounders (four next to each other to average out transversal variations). Subsequently, net sediment transport rates $\left\langle q_{\mathrm{s}}\right\rangle\left(\mathrm{m}^{2} / \mathrm{s}\right)$ at the position of the instrument frame $\left(x_{2}\right)$ were determined from sand volume conservation by spatial integration of the changes in bed level $z_{\mathrm{b}}$ between successive profile measurements:

$$
\left\langle q_{s}\right\rangle_{x 2}=-\int_{x 1}^{x 2} \frac{\partial\left((1-\varepsilon) z_{b}\right)}{\partial t} d x
$$

This integration started at $x_{1}$, a location with zero transport in a fixed bed zone offshore. Because the value and potential variation of porosity $\varepsilon$ during the tests were unknown, a constant value of $\varepsilon=0.4$ was assumed following Dohmen-Janssen and Hanes [2002]. Repetition of the procedure for the multiple experimental runs resulted in an average transport rate and standard deviation for each condition.

In addition to transport rates from Schretlen [2012], the model-data comparison on sediment transport also includes transport rates from the full scale wave flume experiments of DohmenJanssen and Hanes [2002]. In these experiments, again $T$ and $H$ of the nearly cnoidal waves were varied and water depth $h$ was $3.5 \mathrm{~m}$. The horizontal sand bed consisted of well-sorted grains with $d_{50}$ $=0.240 \mathrm{~mm}$ and the horizontal velocities were measured with an Acoustic Doppler Velocimeter (ADV) at around $100 \mathrm{~mm}$ above the still bed level. To the best of our knowledge, we thus include all available transport rates from full scale wave flume experiments on sheet-flow sand transport beneath regular waves. Considering the discussion on different trends in transport between flume and tunnel experiments, also tunnel experiments on transport of fine $\left(d_{50} \leq 0.140 \mathrm{~mm}\right)$ and medium sized $\left(d_{50} \geq 0.210 \mathrm{~mm}\right)$ sand beneath velocity skewed oscillatory flow have been included in the model validation. An overview of all the data used is given in Table 3.1. This table gives the names of the various conditions as used by the original authors, the period $T$, median grain size $d_{50}$, measured transport rates $\left\langle q_{\mathrm{s}}\right\rangle$ and a characterization of the flow velocities at $z=z_{\text {match }}$, where $z_{\text {match }}$ is the level at which the model will be forced to match the measured velocities. Note that flow and transport information generally concern averaged values over multiple runs per condition. For the experiments of Schretlen [2012], standard deviations are given in Table 3.2. 


\subsubsection{Model-data comparison on horizontal velocities}

For model-data comparison on boundary layer flow, we simulate the experiments of Schretlen [2012] by forcing the model at $z=z_{\text {match }}$ with the UVP-measured velocity at that level and compare model and data for the flow underneath. Except for the few runs for which the UVP-data did not extend up to there, we choose the matching level $z_{\text {match }}$ at $40 \mathrm{~mm}$ above the initial still bed level $(z=$ $0 \mathrm{~mm}$ ). Figure 3.1 presents measured and simulated horizontal velocities for a single run of condition $1065 \mathrm{f}$ (harmonic representation). The results for amplitude and phase of the harmonic components, especially component 1 and 2 , show that the model gives a good reproduction of the wave boundary layer thickness: the levels of maximum amplitude in data and model results nearly coincide and model and data show a similar level for the start of the phase lead of the boundary layer flow. A typical characteristic of sheet-flow beneath velocity-skewed waves is deeper mobilization of the bed during the onshore movement compared to the offshore movement (erosion-depth asymmetry). This results in distinct onshore wave averaged velocities $U_{0}$ in the lower part of the sheet-flow layer, which increase with increasing velocity skewness. This onshore mean velocity below the initial bed level is also visible in the shown data. The present model has a fixed bottom level and will therefore not reproduce this specific feature. However, the reproduction of magnitude, direction and shape of the $U_{0}$ profile higher up in the wave boundary layer is remarkably good. To illustrate the quality of this reproduction and the added value of the present model formulations compared to models in the literature, we compare the present model (BL2-SED) with results from respectively the first order 'tunnel' version (BL1-SED), and the purely hydrodynamic version of the present model (BL2-HYDRO) discussed in Kranenburg et al. [2012]. The results of the latter are
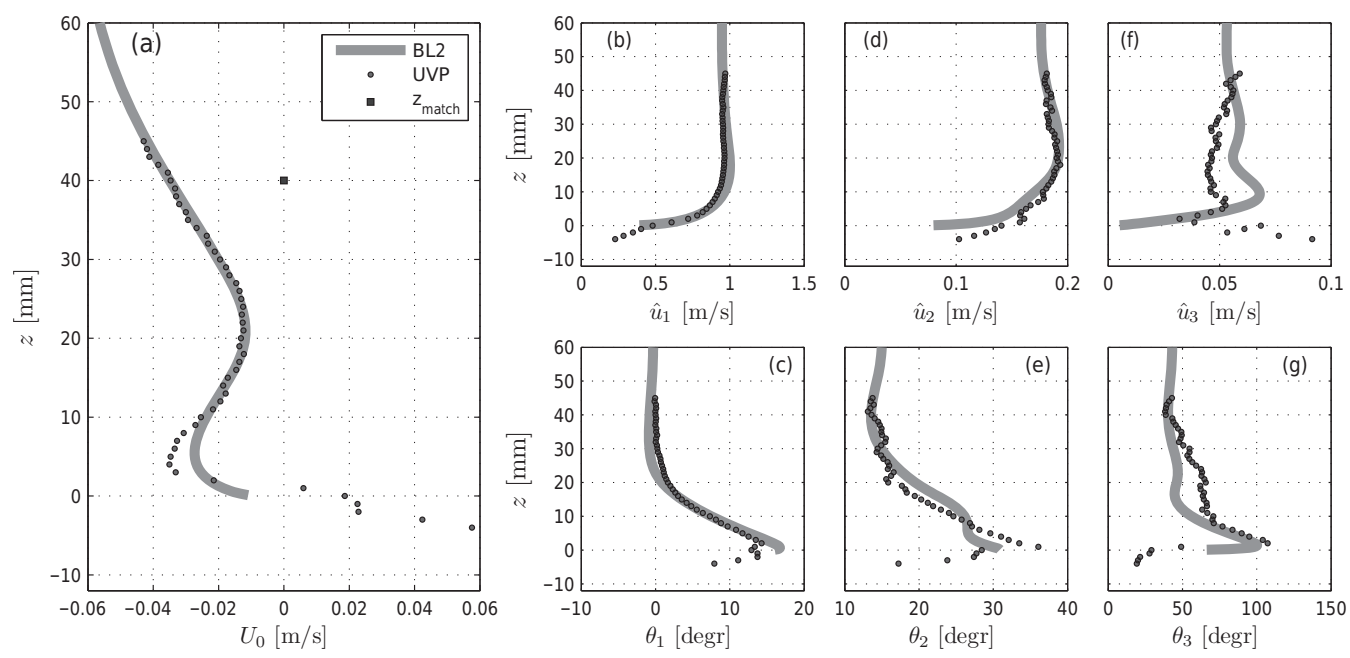

Figure 3.1: (Panel a) Wave averaged velocity $U_{0}$ and (panel b,d,f) amplitudes $\hat{u}$ and (panel c,e,g) phases $\theta$ of first, second and third harmonic components of the horizontal velocity. Dots: experimental data from Schretlen [2012] (condition 1065f: regular velocity-skewed waves with $H=1.0 \mathrm{~m}, T=6.5 \mathrm{~s}, h=3.5 \mathrm{~m}$ and $d_{50}=0.138$ $\mathrm{mm}$ ). Gray line: model results; square: matching level. Positive velocities are directed onshore. 


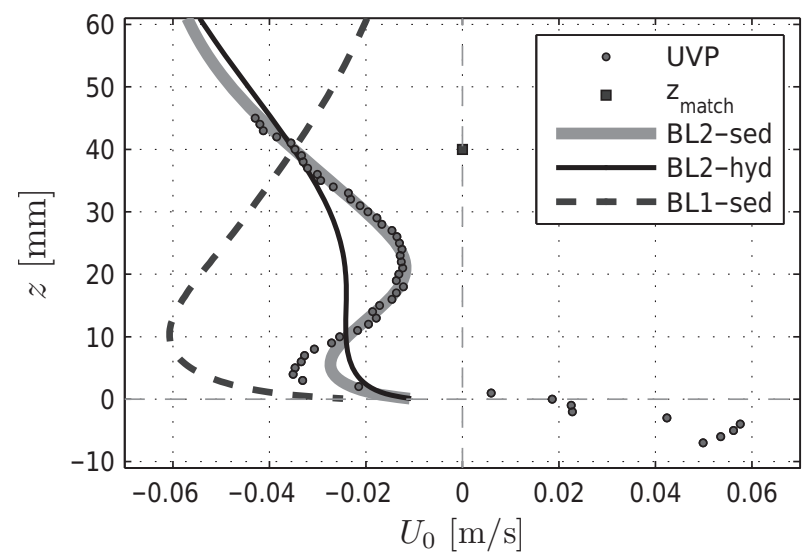

Figure 3.2: Wave averaged horizontal velocity $U_{0}$. Dots: experimental data; lines: simulation with BL1-SED, the first order boundary layer model with suspended sediment; with BL2-HYDRO, the second order boundary layer model without feedback of sediment on the flow; and with BL2-SED, the present second order boundary layer model with suspended sediment. Conditions as in Figure 3.1.

expected to be comparable with Henderson et al. [2004], a second order boundary layer model without feedback of sediment on the flow. For the three model versions, the mismatch between model and data, averaged over the domain between $z=z_{\text {match }}$ and $z=0 \mathrm{~mm}$, computed discretely by:

$$
\frac{1}{z_{\text {match }}} \int_{z=0}^{z=z_{\text {match }}} \sqrt{\left(U_{0, \text { comp }}(z)-U_{0, \text { meas }}(z)\right)^{2}} d z \text {, }
$$

is respectively $0.0292 \mathrm{~m} / \mathrm{s}$ (BL1-SED), $0.0079 \mathrm{~m} / \mathrm{s}$ (BL2-HYDRO) and $0.0024 \mathrm{~m} / \mathrm{s}$ (BL2-SED). The present model not only has by far the smallest averaged mismatch, Figure 3.2 shows that it also gives a better reproduction of the shape of the current profile. We therefore conclude that both progressive wave streaming and feedback of sediment on the flow through stratification effects need to be considered to model the net current in the boundary layer under waves above a mobile bed and to study the influence of streaming on sediment transport.

Figure 3.3 shows $U_{0}$ profiles for experimental conditions with varying $H, T$ and $d_{50}$. The changes in $U_{0}$ for changing $H, T$ and $d_{50}$ in the six runs shown here are representative for the $H, T$ and $d_{50}$ dependency in all other runs, as can be verified for $U_{0}$ at $z_{\text {match }}$ from Table 3.1. These results show that also for different wave and bed conditions the model is rather well able to reproduce the magnitude and shape of the $U_{0}$ profile and also shows a $H, T$ and $d_{50}$ dependency comparable to the data. (Compare e.g. the changes in local minima and maxima with changing $H$ and $T$ ). For more discussion on the shape of the $U_{0}$-profiles, the influence thereon of wave shape streaming, progressive wave streaming and Stokes drift compensation, and the changing balance between these mechanisms for changing wave and bed conditions, we refer to Kranenburg et al. [2012] and Schretlen [2012]. 

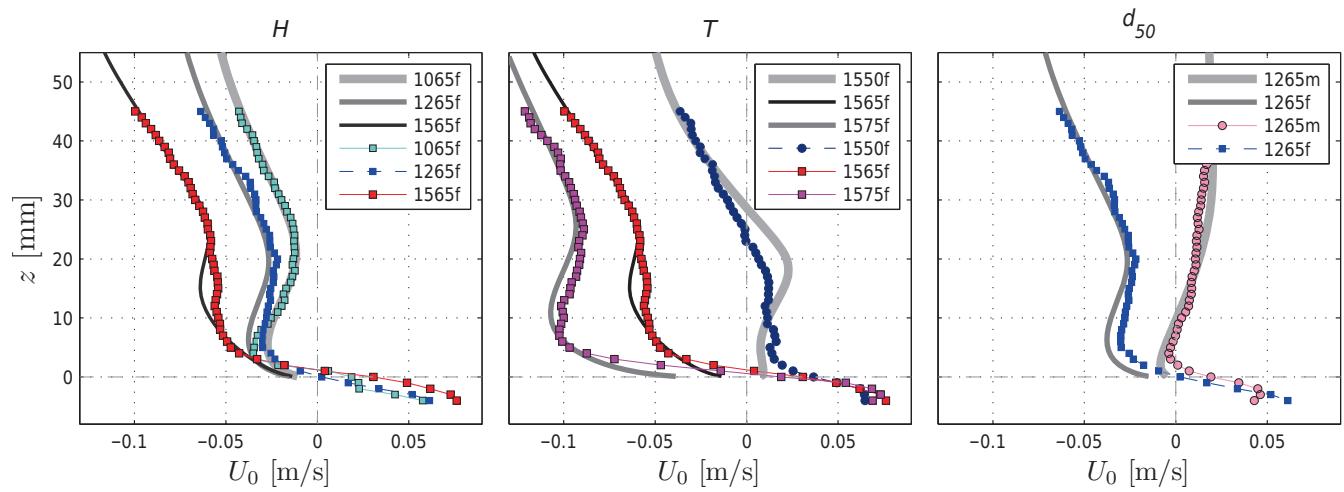

Figure 3.3: Measured and computed profiles of period averaged horizontal velocity $U_{0}$ for various wave and bed conditions. a) for waves with height $H$ of $1.0,1.2$ and $1.5 \mathrm{~m}$; b) for waves with period $T$ of 5.0, 6.5 and $7.5 \mathrm{~s}$; c) for waves over beds with a median grain size $d_{50}$ of 0.138 (f, fine) and $0.245 \mathrm{~mm}$ (m, medium).

\subsubsection{Model-data comparison on sediment transport}

Next, we compare computed and measured net sediment transport rates. Note that not every experimental run of Schretlen [2012] resulted in successful measurement of both velocity and sediment transport. To include as much experimental information as possible, the set-up of the comparison is as follows: for each run with successful UVP measurements, a simulation is carried out, using the UVP-measured velocity signal at $z=z_{\text {match }}$ to drive the model. All these simulations result in a single computed net sediment transport rate. Per wave condition, we determine mean and standard deviation of the computed transport rates and compare these with the mean and standard deviation of the experimentally determined transport rates. Note that the latter thus also includes runs for which no UVP measurements are available, while the computed results also include runs for which no transport rate could be determined from the experiments. The flume experiments of Dohmen-Janssen and Hanes [2002] $\left(d_{50}=0.240 \mathrm{~mm}\right)$ are simulated by driving the model with the ADV-measured horizontal velocities at around $100 \mathrm{~mm}$ above the still bed level (i.e around 2.5 times the UVP-matching level). For these experiments, no velocity data are available closer to the bed and per condition only one time series of horizontal velocities is available. As a consequence, the computed net transport for these conditions is based on one simulation only, while the measured transport is an average over multiple experimental runs. The model-data comparison on net transport rates $\left\langle q_{\mathrm{s}}\right\rangle$ is shown in Figure 3.4a. Figure 3.4b extends Figure 3.4a with simulations of tunnel experiments on transport of both fine $\left(d_{50} \leq 0.140 \mathrm{~mm}\right)$ and medium $\left(d_{50} \geq 0.210 \mathrm{~mm}\right)$ sand under velocity-skewed oscillations. The (mean) computed net transport rates per condition have been added to Table 3.1. For the conditions of Schretlen [2012] standard deviations have been added to Table 3.2. 


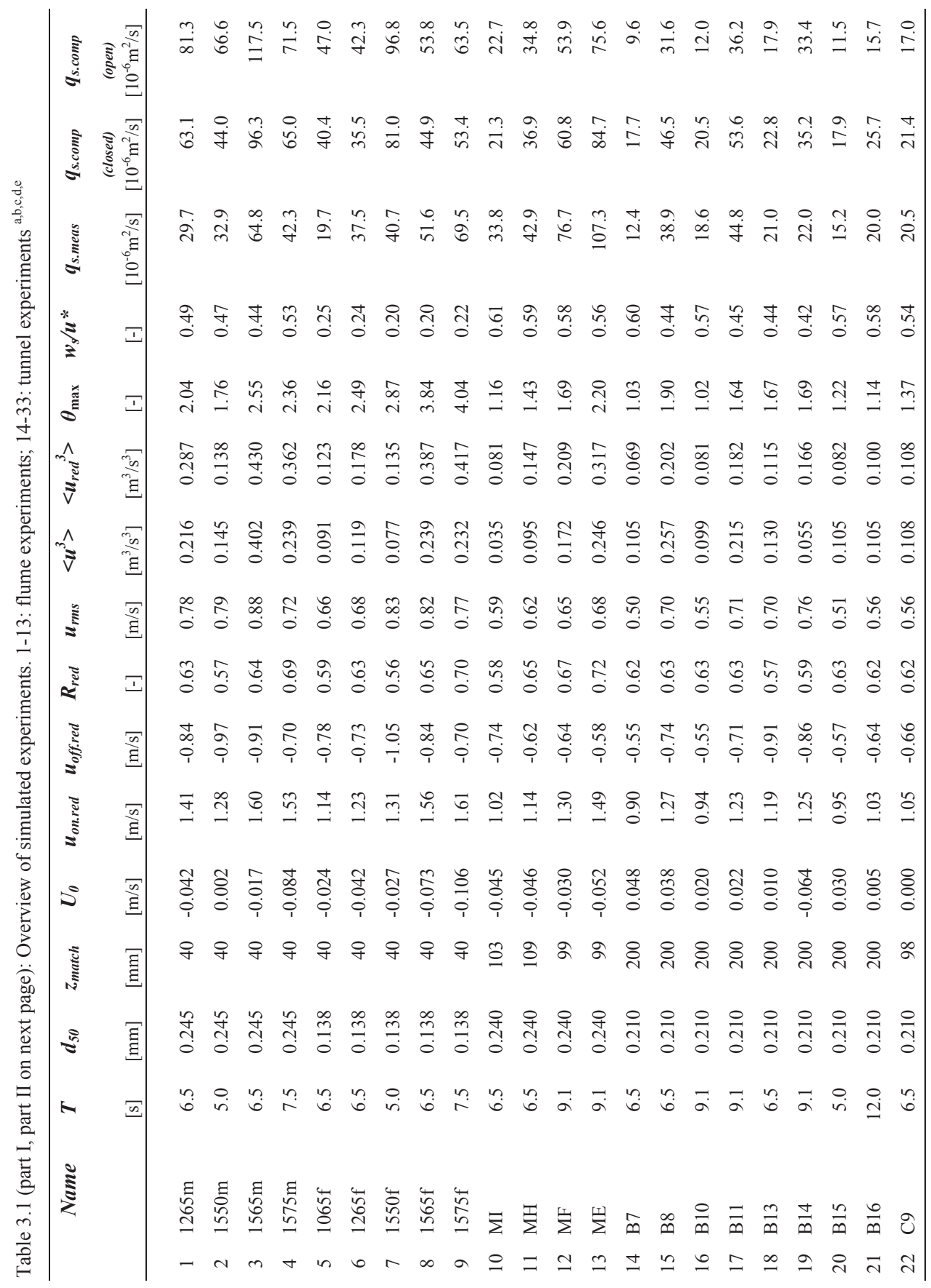




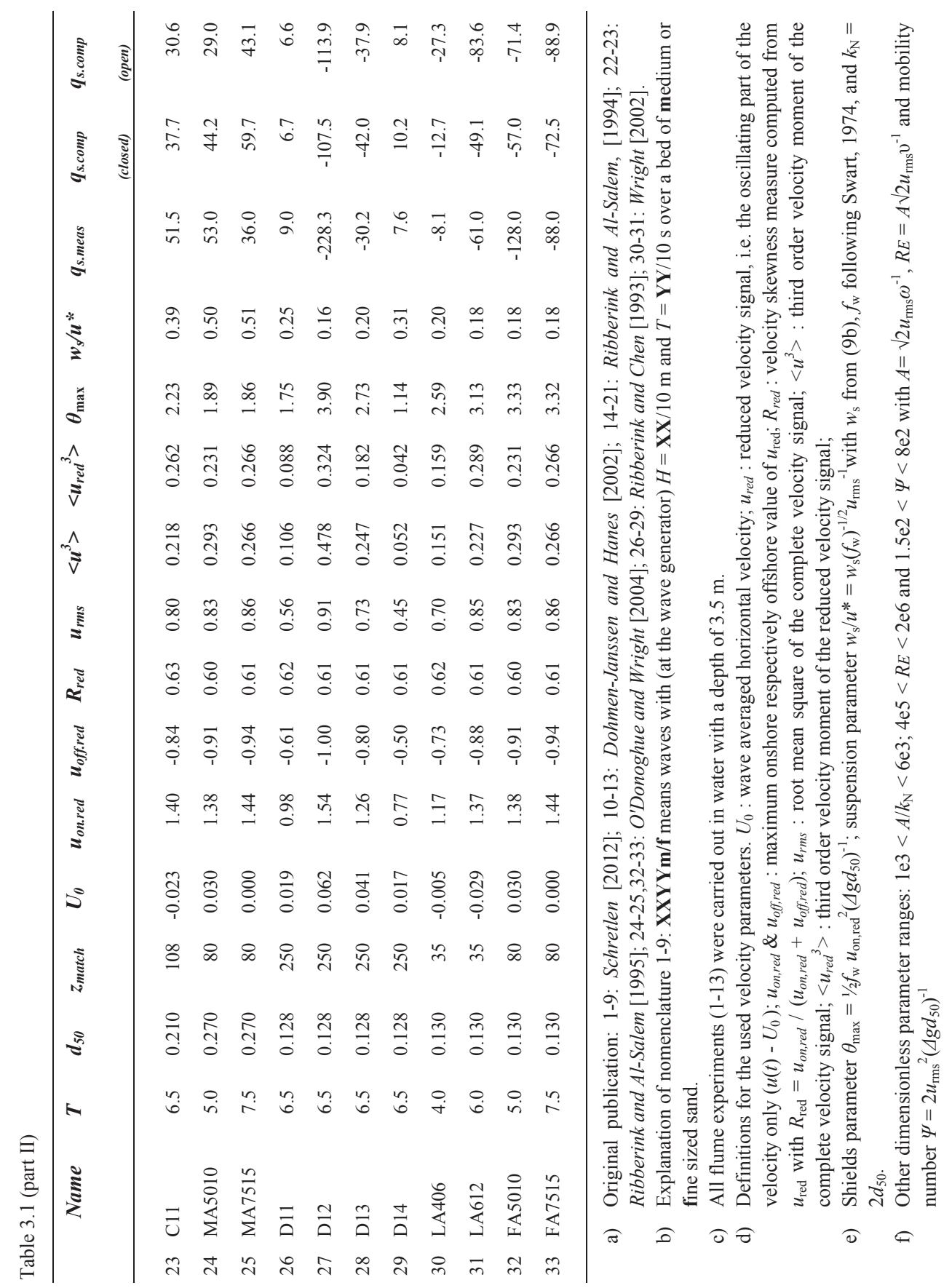



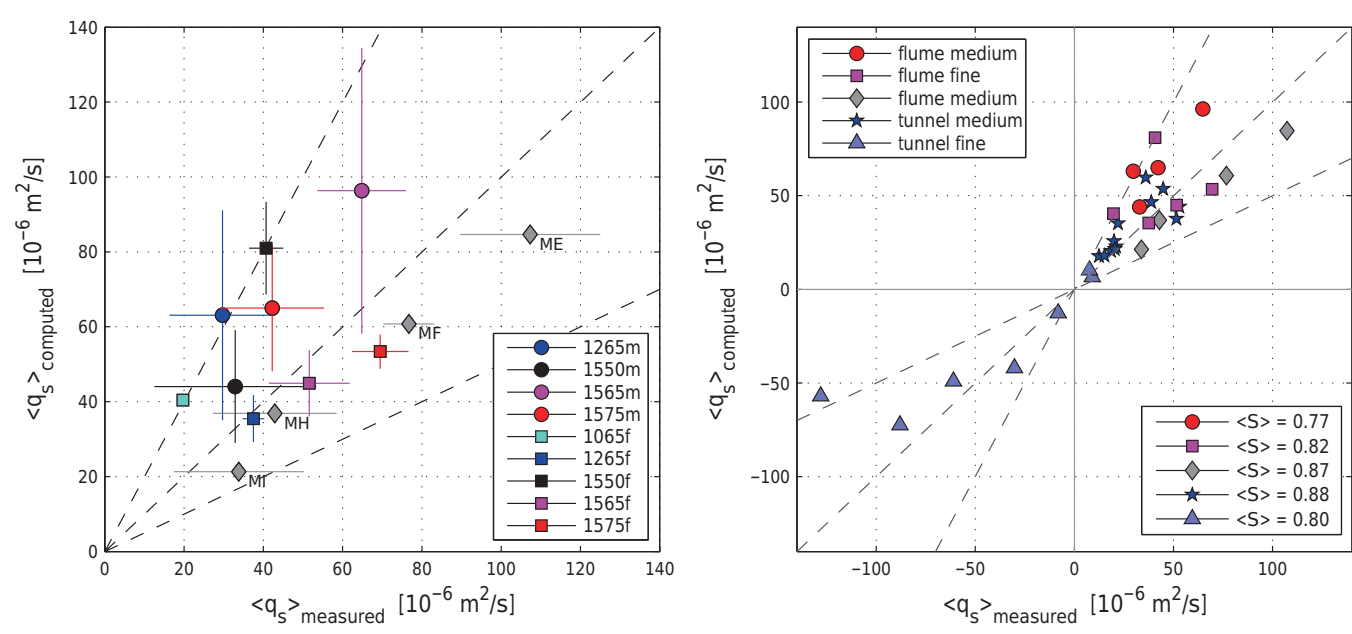

Figure 3.4: Computed against measured net sediment transport rates $\left\langle q_{\mathrm{s}}\right\rangle$ under regular, predominantly velocity-skewed waves. Left) For all available full scale flume experiments, with standard deviations; Right) for both flume and tunnel experiments. Circles: Schretlen [2012] flume experiments with medium sized sand (nr. 1-4 in Table 3.1); squares: Schretlen [2012] flume experiments with fine sand (5-9); diamonds: DohmenJanssen and Hanes [2002] flume experiments with medium sized sand (10-13); stars (right panel only): tunnel experiments with medium sand (14-25); triangles (right panel only): tunnel experiments with fine sand (2633); a total of 33 conditions and 65 simulations (note that condition 27 falls outside the graph). Dashed lines: $y$ $=\alpha x$, for $\alpha$ is $1 / 2,1$ and $2 ;<S>$ gives a reproduction quality measure per set, see eq. (3.18).

We observe from Figure 3.4 that the direction of $\left\langle q_{\mathrm{s}}\right\rangle$ is reproduced correctly in all cases. For nearly all cases, the model prediction is within a factor 2 of the measured $\left\langle q_{\mathrm{s}}\right\rangle$. We see in panel a) that within the various sets of wave flume experiments, also trends of increasing transport are reproduced, except for condition 1065f, $1550 \mathrm{f}$ and $1265 \mathrm{~m}$. For each set, a score has been given to the reproduction by averaging $S$ over all cases within the set, with:

$$
S=1-\frac{\left|q_{s, c}-q_{s, m}\right|}{\left|q_{s, c}+q_{s, m}\right|}
$$

This measure results in identical scores for over prediction with a factor 2 and under prediction with a factor $1 / 2$ (namely 0.667 ), and results in negative values when the transport direction is not reproduced well. The results per set are added to Figure 3.4 and all lie between 0.77 and 0.88 (around factor 1.6 and 1.3), which is considered a good quantitative reproduction for sediment transport rates [Davies et al., 2002]. The model overpredicts the medium sand flume experiments of Schretlen [2012] (circles, $\langle S\rangle=0.77$ ), while it slightly underpredicts the medium sand flume experiments of Dohmen-Janssen and Hanes [2002] (diamonds, $\langle S\rangle=0.87$ ). An explanation for this systematic difference might be the wider sieve curve of the sand in the experiments of Schretlen [2012], a difference not present in the simulations because the model considers the median grain size only. Finally, note that for the medium sand flume experiments of Schretlen [2012] the differences between the various runs of a condition are rather large. This experimental scatter is present both for 
the UVP-measured velocities (input to the model) and the measured (and computed) transport rates (see Table 3.2).

\subsubsection{Transport against velocity moments}

An important observation from tunnel experiments with velocity skewed oscillatory flows is that the net transport rate of medium sized sand $\left(d_{50} \geq 0.2 \mathrm{~mm}\right)$ is proportional to the third-order moment of the horizontal velocity in the free stream: $\left\langle q_{\mathrm{s}}\right\rangle \sim\left\langle u^{3}\right\rangle$ [Ribberink and Al-Salem, 1994]. This relation, an indication for quasi-steady behavior of $\left\langle q_{\mathrm{s}}\right\rangle$ during the wave cycle (see e.g. Bailard [1981]), is not valid for finer sands [O'Donoghue and Wright, 2004]. In that case, phase-lag effects will play a role and instantaneous concentration and intra wave transport are no longer coupled to the instantaneous free stream velocity. Net transport rates can even become negative for increasing positive velocity moments $\left\langle u^{3}\right\rangle$. In wave flume experiments, the $\left\langle q_{\mathrm{s}}\right\rangle \sim\left\langle u^{3}\right\rangle$ relation for medium sized sand is also found [Dohmen-Janssen and Hanes, 2002]. However, Schretlen [2012] show that the reversal of transport direction for fine sand is absent. Before we apply the model to investigate physical explanations of these differences, we need to verify the model reproduction of these trends.

In Figure 3.5, $\left\langle q_{\mathrm{s}}\right\rangle \sim\left\langle u^{3}\right\rangle$ trends from experiments (column 1) are compared with the simulation results (column 2), both for medium (panels a) and fine (panels b) sand and for tunnel as well as flume conditions (different symbols). We choose to determine the third order velocity moment from the oscillating part of the horizontal velocities only $\left(u_{\mathrm{red}}=u(t)-U_{0}\right.$, see Table 3.1). The reason is that $<u^{3}>$ is sensitive for $U_{0}$-variations, while $U_{0}$ depends on the height of the velocity measurements (much more than the oscillating velocity, see e.g. Figure 3.1), and is itself affected by the differences between flume and tunnel. In this way differences in $z_{\text {match }}$ between the various experiments will not influence the trends and tunnel and flume experiments that physically model the same wave condition will have identical third order velocity moments.

Panel $1 \mathrm{~b}$ clearly shows the differences in transport of fine sand between tunnel and flume experiments: in the tunnel, the transport direction reverses from onshore to offshore with increasing $<u_{\text {red }}{ }^{3}>$. For the flume cases, the transport remains onshore. Panel $2 \mathrm{~b}$ shows that these trends are reproduced by the model. Also the moment of transition from onshore to offshore transport for fine sand $\left(<u_{\text {red }}{ }^{3}>\approx 0.15 \mathrm{~m}^{3} / \mathrm{s}^{3}\right)$ is predicted correctly. Like in the experiments, the simulated transport rates of medium sized sand (panels a) are also generally increasing with increasing $\left\langle u_{\text {red }}{ }^{3}\right\rangle$ (panel $2 b$ ). The experimental results show both trends for larger (diamonds, measurements of [DohmenJanssen and Hanes, 2002]) as well as smaller (circles, [Schretlen, 2012]) net transport rates in wave flumes compared to tunnels (stars) for identical $<u_{\text {red }}{ }^{3}>$. The accompanying model simulations (panel $2 \mathrm{a}$ ), can be represented well with one simple third-order power function $\left\langle q_{\mathrm{s}}\right\rangle=A\left\langle u_{\text {red }}{ }^{3}\right\rangle$. Again, this might be explained by a systematic difference between the two series of medium sand flume experiments, not reflected by the model, that results in generally smaller measured net transport rates in the experiments of Schretlen [2012] compared to Dohmen-Janssen and Hanes [2002]; a possible explanation is the sieve curve width. See Schretlen [2012] for further discussion on the experimental differences. 

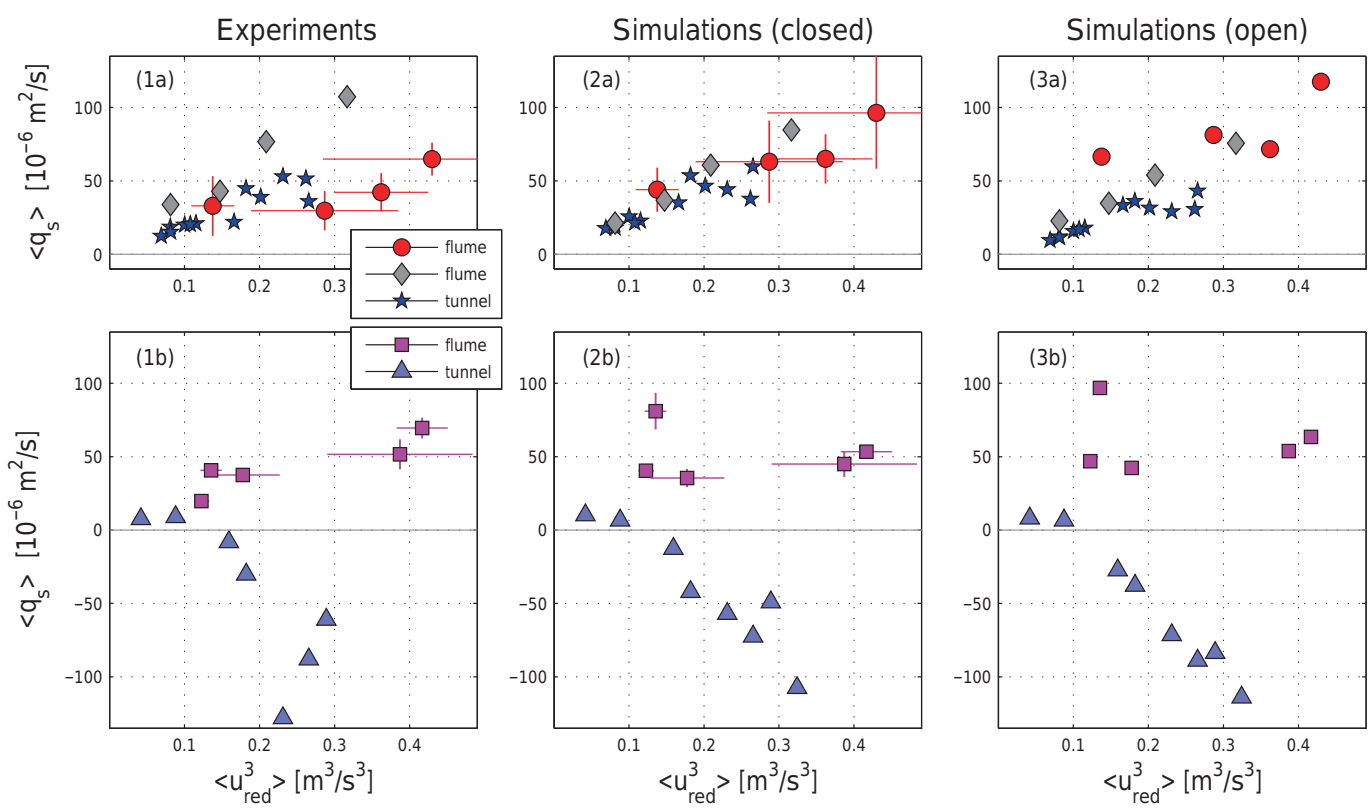

Figure 3.5: Measured (1a,b) and computed (2a,b) net sediment transport rates $\left\langle q_{\mathrm{s}}\right\rangle$ of medium (panels a) and fine (panel b) sands against the third order velocity moment as determined from the oscillating part of the horizontal velocity $u_{\mathrm{red}}$, for all conditions in Table 3.1. Panel 3a\&b: results for simulations without compensation of mass transport in flume and tunnel (section 3.4.1).

Table 3.2: Standard deviations of velocity and transport parameters for the Schretlen [2012] experiments and accompanying simulations;

\begin{tabular}{|c|c|c|c|c|c|c|c|c|c|c|c|c|}
\hline \multicolumn{2}{|c|}{ Condition } & $n^{a}$ & $\begin{array}{c}\boldsymbol{U}_{\boldsymbol{0}} \\
{[\mathrm{m} / \mathrm{s}]}\end{array}$ & $\begin{array}{c}\boldsymbol{U}_{\text {on,red }} \\
{[\mathrm{m} / \mathrm{s}]}\end{array}$ & $\begin{array}{c}\boldsymbol{U}_{\text {off,red }} \\
{[\mathrm{m} / \mathrm{s}]}\end{array}$ & $\begin{array}{c}\boldsymbol{R}_{\text {red }} \\
{[-]}\end{array}$ & $\begin{array}{l}\boldsymbol{u}_{r m s} \\
{[\mathrm{~m} / \mathrm{s}]}\end{array}$ & $\begin{array}{r}<\boldsymbol{u}^{3}> \\
{\left[\mathrm{m}^{3} / \mathrm{s}^{3}\right]}\end{array}$ & $\begin{array}{c}<\boldsymbol{u}_{\text {red }}{ }^{3}> \\
{\left[\mathrm{m}^{3} / \mathrm{s}^{3}\right]}\end{array}$ & $\begin{array}{c}\boldsymbol{q}_{\mathbf{s , \text { meas }}} \\
(\text { other } n) \\
{\left[10^{-6} \mathrm{~m}^{2} / \mathrm{s}\right]}\end{array}$ & $\begin{array}{c}q_{s, \text { comp }} \\
\text { (closed) } \\
{\left[10^{-6} \mathrm{~m}^{2} / \mathrm{s}\right]}\end{array}$ & $\begin{array}{c}\boldsymbol{q}_{\mathbf{s , c o m p}} \\
\text { (open) } \\
{\left[10^{-6} \mathrm{~m}^{2} / \mathrm{s}\right]}\end{array}$ \\
\hline 1 & $1265 \mathrm{~m}$ & 5 & 0.044 & 0.17 & 0.09 & 0.01 & 0.09 & 0.124 & 0.098 & 13.4 & 28.0 & 32.2 \\
\hline 2 & $1550 \mathrm{~m}$ & 7 & 0.034 & 0.13 & 0.15 & 0.02 & 0.10 & 0.079 & 0.029 & 20.4 & 15.1 & 26.9 \\
\hline 3 & $1565 \mathrm{~m}$ & 4 & 0.034 & 0.18 & 0.08 & 0.01 & 0.09 & 0.220 & 0.146 & 11.2 & 38.2 & 37.4 \\
\hline 4 & $1575 \mathrm{~m}$ & 4 & 0.027 & 0.10 & 0.07 & 0.01 & 0.04 & 0.084 & 0.063 & 13.0 & 16.8 & 15.5 \\
\hline 5 & $1065 f$ & 3 & 0.008 & 0.04 & 0.03 & 0.00 & 0.02 & 0.006 & 0.010 & 1.8 & 2.0 & 4.0 \\
\hline 6 & $1265 f$ & 7 & 0.011 & 0.11 & 0.09 & 0.01 & 0.06 & 0.047 & 0.049 & 2.8 & 6.3 & 6.7 \\
\hline 7 & $1550 \mathrm{f}$ & 4 & 0.011 & 0.04 & 0.05 & 0.01 & 0.03 & 0.020 & 0.014 & 4.3 & 12.4 & 18.4 \\
\hline 8 & $1565 \mathrm{f}$ & 5 & 0.024 & 0.13 & 0.12 & 0.02 & 0.08 & 0.077 & 0.097 & 10.2 & 8.8 & 11.1 \\
\hline 9 & $1575 \mathrm{f}$ & 2 & 0.003 & 0.06 & 0.07 & 0.01 & 0.04 & 0.010 & 0.034 & 7.1 & 4.6 & 0.3 \\
\hline
\end{tabular}

a) number of UVP-velocity signals, also input to $n$ simulations (see p.149 for the UVP run id's). 


\subsubsection{Sensitivity analysis and discussion}

We conclude the validation with a sensitivity analysis and discussion on the modeling concept. The sensitivity analysis focuses on model formulations for mixing, roughness and hindered settling. Although the present choices for $\sigma_{\mathrm{t}}, k_{\mathrm{N}}$ and $w_{\mathrm{s}}$ find their basis in literature, their application for sheetflow under waves is not without discussion. Nielsen et al. [2002] e.g. questioned the eddy diffusivity concept and found a settling velocity reduction significantly stronger than predicted by Richardson and Zaki [1954]. Next, some authors have suggested modeling flow over mobile beds using much larger $k_{\mathrm{N}}$ values (e.g. Sumer et al. [1996], Dohmen-Janssen and Hanes [2002]) or use $k_{\mathrm{N}}$ as a $d_{50}$ independent tuning parameter [Ruessink et al., 2009]. Here we investigate the effect of decreasing/increasing $\sigma_{\mathrm{t}}, k_{\mathrm{N}}$ and $p$ (hindered settling effect, equation (3.9)) with a factor of about 1.5. In addition we test for $k_{\mathrm{N}}$ increased one order of magnitude (test 5). The tests and results are presented in Figure 3.6 and Table 3.3.

(a)

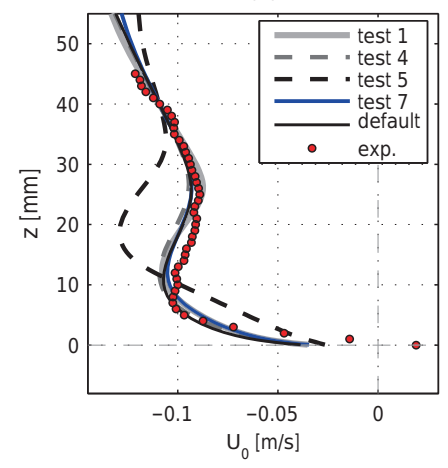

(b) test 1

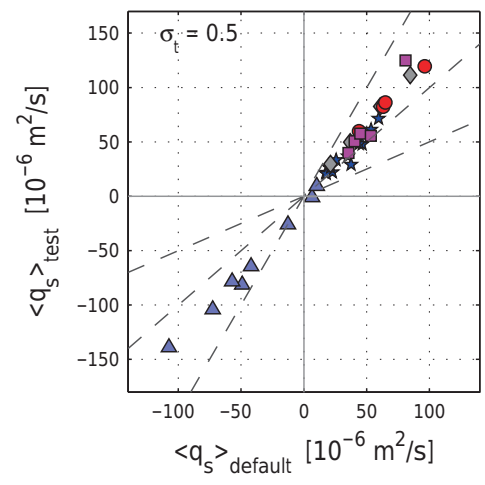

(c) test 5

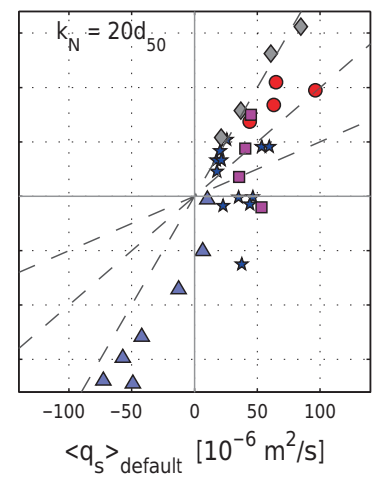

(d) test 7

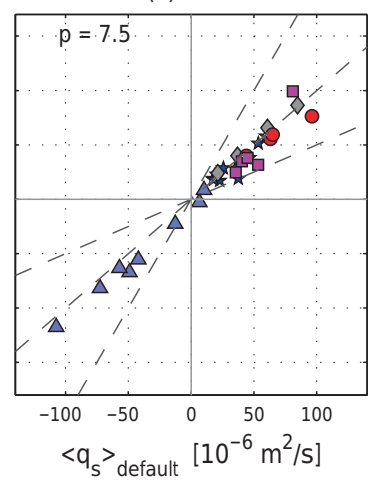

Figure 3.6: Results from the sensitivity analysis for a selection of tests from table 3. Panel (a): measured and computed mean current velocity $U_{0}$; panel (b-d): Transport rate $\left\langle q_{\mathrm{s}}\right\rangle$ computed with adapted model parameter values against $\left\langle q_{\mathrm{s}}\right\rangle$ computed with the original values, for all conditions of Table 1. (Default values: $\sigma_{\mathrm{t}}=0.7, k_{\mathrm{N}}=2 d_{50}, p=5.0$ ). Dashed lines: $y=\alpha x$, for $\alpha$ is $1 / 2,1$ and 2 . 
Table 3.3: Sensitivity Tests. Data reproduction quality measure $\langle S>$ for all tests, both per set and total.

\begin{tabular}{|c|c|c|c|c|c|c|c|c|c|}
\hline Set & nr & test $0^{\mathrm{a}}$ & $\begin{array}{c}\text { test } 1 \\
\sigma_{\mathrm{t}}=0.5\end{array}$ & $\begin{array}{r}\text { test } 2 \\
\sigma_{\mathrm{t}}=1.0\end{array}$ & $\begin{array}{c}\text { test } 3 \\
k_{\mathrm{N}}=1.3 d_{50}\end{array}$ & $\begin{array}{r}\text { test } 4 \\
k_{\mathrm{N}}=3 d_{50}\end{array}$ & $\begin{array}{c}\text { test } 5 \\
k_{\mathrm{N}}=20 d_{50}\end{array}$ & $\begin{array}{l}\text { test } 6 \\
p=3.3\end{array}$ & $\begin{array}{l}\text { test } 7 \\
p=7.5^{b}\end{array}$ \\
\hline flume medium & $1-4$ & 0.77 & 0.65 & 0.91 & 0.80 & 0.74 & 0.64 & 0.79 & 0.84 \\
\hline flume fine & $5-9$ & 0.82 & 0.77 & 0.85 & 0.85 & 0.78 & 0.40 & 0.83 & 0.71 \\
\hline flume medium & $10-13$ & 0.87 & 0.95 & 0.70 & 0.80 & 0.93 & 0.76 & 0.80 & 0.90 \\
\hline tunnel medium & $14-25$ & 0.88 & 0.82 & 0.92 & 0.91 & 0.85 & -0.50 & 0.90 & 0.85 \\
\hline tunnel fine & $26-33$ & 0.80 & 0.63 & 0.61 & 0.79 & 0.72 & 0.23 & 0.69 & 0.56 \\
\hline all conditions & $1-33$ & 0.84 & 0.76 & 0.81 & 0.85 & 0.81 & 0.10 & 0.82 & 0.77 \\
\hline
\end{tabular}

a) Test 0: reference model parameter choices with $\sigma_{\mathrm{t}}=0.7 ; k_{\mathrm{N}}=2.0 d_{50} ; p=5.0$

b) A larger $p$ leads to increased effects of hindered settling

Firstly, we observe from panel (a) that $U_{0}$ is only marginally affected by factor 1.5 changes in $\sigma_{\mathrm{t}}, k_{\mathrm{N}}$ and $p$. However, the order of magnitude change in $k_{\mathrm{N}}$ (test 5 ) introduces a large overestimation of the level and magnitude of the maximum offshore boundary layer streaming. This results from increasing boundary layer thickness with increasing roughness, see also the model behavior tests in Kranenburg et al. [2012] (BL2-HYDRO). For a selection of tests, panel $b$ - $d$ show $<q_{\mathrm{s}}>$ computed with adapted model parameters against $\left\langle q_{\mathrm{s}}\right\rangle$ computed with the original values for the conditions of Table 3.1. By and large, test 1 (reduced $\sigma_{\mathrm{t}}$, increased mixing) shows an increase of the absolute transport rates for all sets. In test 7 (increased $p$, increased hindered settling effect), the results for medium sized grains (circles, diamonds, starts) are nearly unaltered, while the fine sand cases (generally) show a slightly increased transport in offshore direction. Apparently, phase-lags effects increase in both tests, while the stronger mixing also strengthens the onshore transport mechanisms. The changes for $\left\langle q_{\mathrm{s}}\right\rangle$ in test 5 ( $k_{\mathrm{N}}$ increased with a factor 10) are clearly of another order of magnitude. Both for the sets with medium sand in a flume (circles, diamonds) and with fine sand in a tunnel (triangles), $\left|\left\langle q_{\mathrm{s}}\right\rangle\right|$ increases drastically. The two other sets show completely scattered results, from an increase with a factor 2 to a reversal of the transport direction. Table 3.3 lists the consequences for model-data comparison for all sensitivity tests. Clearly, from $U_{0}$ and $\left\langle q_{\mathrm{s}}\right\rangle$ results, there is no need to adopt alternative formulations.

A more fundamental question is whether it is justified to model sheet-flow as sand in suspension. Firstly, note that based on the non-dimensional parameters $\theta$ and $w_{\mathrm{s}} / u_{*}$ in Table 3.1 all experimental conditions can be classified as well inside the domain of "suspension mode sheet-flow" (Wilson [1989]: sheet-flow for $\theta>0.8$; Sumer et al. [1996]: suspension mode for $\left.w_{\mathrm{s}} / u_{*}<0.8-1.0\right)$. Also regarding the classical distinction between bed load and suspended load, the Rouse number $P=$ $w_{\mathrm{s}}(\kappa u *)^{-1}$ indicates that suspension load transport will dominate by far in most cases. Indeed Hassan and Ribberink [2010], who used a suspension model with a bed load formula to model the flux beneath $z=2 d_{50}$, found the bed load component of minor importance for the total computed 
transport (except for their large grain test). Furthermore, although shifted to levels above $\mathrm{z}=0 \mathrm{~mm}$ (instead of below $\mathrm{z}=0$ as measured in the pick-up layer), also the shape and magnitude of the net flux profiles were reproduced very well. Apparently, the sheet-flow layer dynamics can to a certain extent be represented as an advection-diffusion process, with the present empirical model for reference concentration (neglecting the details of sediment entrainment and dynamics in concentrations close to the pack limit). Based on the validation results and the considerations above, we consider the suspension approach appropriate for the present research. More detailed investigation on erosion behavior and sheet-flow layer thickness would require further development and application of other modeling concepts, e.g. two-phase models.

\subsection{RELATIVE IMPORTANCE OF VARIOUS FREE SURFACE EFFECTS}

This section describes model simulations to investigate the relevance of the hydrodynamic differences between tunnel and flume experiments for sediment transport rates. We firstly study the role of contrasting return flow mechanisms in the two experimental settings (3.4.1). Subsequently, we focus on differences induced by advection processes inside the wave boundary layer. Their effects on sediment transport are illustrated with a discussion on velocities and concentrations beneath sinusoidal waves in section 3.4.2 and quantified for more realistic non-linear waves in section 3.4.3.

\subsubsection{Compensation of mass transport in closed tunnels and flumes}

In a closed tunnel, the offshore wave shape streaming will cause an onshore directed mass transport compensation current. The strength of this current not only depends on the streaming, but also on properties of the facility like height and width. Beneath progressive surface waves, the mass transport originates not only from wave shape streaming, but also from the onshore progressive wave streaming and especially the onshore Stokes drift. In a flume with closed ends, this will result in a mean pressure gradient driving an offshore directed (Eulerian) compensating current. We determine the influence of these mass compensation mechanisms on sediment transport by comparing the earlier simulations with simulations of hypothetical open facilities, set up as described in section 3.2.2. Since the level $z_{\text {match }}$ of the horizontal velocity measurements used before is practically outside the wave boundary for all used tunnel and flume experiments, we use $u_{\text {red }}$ at $z=z_{\text {match }}$ as input signal to determine the oscillating horizontal pressure gradient. Figure 3.7 shows $<q_{\mathrm{s}}>$ for 'open' versus 'closed' simulations; Panel 3a\&b of Figure 3.5 show the newly computed $<q_{\mathrm{s}}>$ against $<u_{\text {red }}{ }^{3}>$ (identical to $<u_{\text {red }}{ }^{3}>$ for the measurements and closed simulations).

As expected, Figure 3.7 shows that the return flow generally leads to less onshore transport for flume conditions (with offshore directed return current) and to more onshore (or less offshore) transport for tunnel conditions (with onshore directed return current). This influence of the return flow is generally not very large. Panel $3 \mathrm{a} \& \mathrm{~b}$ of Figure 3.5 show that also the $<q_{\mathrm{s}}>-<u_{\text {red }}{ }^{3}>$ trends are not affected significantly. Compared to the closed simulations, the open simulations for medium sand show a more distinct trend for larger transport rates in flumes (both sets) compared to tunnels for identical $<u_{\text {red }}{ }^{3}>$. 




Figure 3.7: Computed net sediment transport rates $\left\langle q_{\mathrm{s}}\right\rangle_{\text {open }}$ versus $\left\langle q_{\mathrm{s}}\right\rangle_{\text {closed }}$ i.e. simulations without the current that compensates the mass transport, versus simulations with this current. Results for all conditions of Table 3.1. Dashed lines: $y=\alpha x$, for $\alpha$ is $1 / 2,1$ and 2 .

\subsubsection{Advection processes: illustration for sinusoidal waves}

Next, we discuss one by one the additional free surface related momentum and sediment advection processes in the horizontally non-uniform wave boundary layer, as present in flume and prototype situation and not in tunnels. These additional horizontal and vertical advection processes each appear in the reduced equations (3.1) or (3.8) in one single advective term (see Table 3.4). We illustrate the effects of these processes on boundary layer velocities and concentrations by comparing simulations with the advective terms one by one switched on to a reference simulation (REF) with all these terms switched off (BL1-model). All simulations are 'open' simulations in which the model is forced with an identical sinusoidal horizontal free stream velocity with amplitude $\hat{u}_{\infty}=1.0 \mathrm{~m} / \mathrm{s}$ and period $T=$ $6.5 \mathrm{~s}$. The simulations have been carried out for water depth $h=3.5 \mathrm{~m}$ and grain size $d_{50}=0.1 \mathrm{~mm}$. The surplus of horizontal velocity and sediment concentration from the various free surface effects is shown in Figure 7 (a-d). Panel (e) and (f) show the vertical profile of the period averaged sediment flux. The resulting net transport rates have been added to Table 3.4. Note that the reference simulation of a sinusoidal oscillating flow yields a zero wave averaged velocity, sediment flux and net transport rate.

We firstly discuss $w \partial u / \partial z$. This single term is the driver of the additional onshore streaming under progressive waves. This occurs through a net downward transport of horizontal momentum into the boundary layer by the vertical orbital motion as a result of the phase shift of the horizontal orbital 
velocities over the boundary layer height. The extra onshore current in the wave boundary layer is clearly visible in the surplus velocities in Figure 3.8 (a). The primary effect of this current is an additional current related (suspended) sediment flux over the whole wave boundary layer. The velocity skewness will also increase. Expected secondary effects are therefore increased pick-up rates under the wave crest and stirring up of sediment to higher levels because of larger flow and turbulence intensities. Under the trough, the opposite will occur.

The vertical orbital motion might also contribute to onshore transport trough vertical sediment advection. The vertical motion introduces a difference between the on- and offshore phase of the wave: at the reversal of the flow from on- to offshore, the orbital motion will be downward, while it will be upward during off- to onshore flow reversal. This becomes relevant for the sediment concentration when grains are stirred up to levels where the vertical velocity $\widetilde{w}$ is in the order of the grain settling velocity $w_{\mathrm{s}}$. In that case, the concentration at this level will decrease faster after the onshore movement and slower after the offshore movement. In other words, the phase-lag between velocity and concentration will behave differently under the wave crest and trough. Figure 3.8 (b) shows the consequences of $w \partial c / \partial z$ for the concentration profiles: under the crest more sediment is present at higher levels, under the trough more sediment is present near the bed. Consequently, positive net sediment fluxes appear higher up in the boundary layer and negative net sediment fluxes appear near the bed. These opposite contributions finally lead to a relatively small influence of vertical sediment advection on the vertically integrated net flux or net transport rate.

Next, in the horizontally non-uniform flow field the advection of sediment by the horizontal orbital motion might also contribute to onshore transport. The horizontal gradients in the sediment flux cause an accumulation of sediment in front of the wave top, where the flux gradient $\partial(u c) / \partial x<0$. Behind the top the opposite occurs. As a result, the absolute rates of change of the sediment concentration are larger and the concentration reacts faster on velocity changes during onshore flow than during offshore flow. A modulation in the concentration takes place, with an amplification of the concentration peak at maximum onshore velocity and a reduction at maximum offshore velocity, see Figure 3.8 (panel d). This induces a net contribution to sediment transport in the onshore direction. An analytical illustration of this process is given in appendix E (considering horizontal sediment exchange only). It shows that the additional net flux due to the modulation is proportional to $\hat{u}^{2} / c_{\mathrm{p}}$. Note that $\hat{u} / c_{\mathrm{p}}$ denotes the order of magnitude of the advective terms compared to the other terms and that the advection terms $w \partial c / \partial z$ and $u \partial c / \partial x$ together describe Stokes' drift of sand in an Eulerian model.

Like the effect of $u \partial c / \partial x$ for sediment, the primary effect of $u \partial u / \partial x$ is an modulation of the horizontal orbital velocities. When forced with a sinusoidal pressure gradient, $u \partial u / \partial x$ would lead to an increased horizontal velocity under the wave top and a decreased velocity under the wave trough (i.e. velocity skewness). However, here we forced the model to match a sinusoidal free stream velocity. As a result, the non-linear term induces slightly acceleration-skewed flow inside the boundary layer (increased acceleration, decreased deceleration). The resulting difference in 
turbulence yields sediment stirring to higher (less high) levels during onshore (offshore) flow, which yields a small positive net sediment fluxes at higher levels, see Figure 3.8 (panel c and e).

The primary effects of the various advection processes beneath progressive waves have been summarized in Table 3.4. Especially $w \partial u / \partial z$ and $u \partial c / \partial x$ have a clear onshore influence on net transport rates trough onshore contribution to the net sediment flux over the entire vertical. The other two terms ( $w \partial c / \partial z$ and $u \partial u / \partial x$ ) lead to both onshore (higher up in the vertical) and offshore fluxes (at lower levels). This results (for these conditions) in only small effects on the net sediment transport. It is also shown that the contribution from $u \partial c / \partial x$ to the net flux is nearly entirely wave-related $(<\tilde{u} \tilde{c}>)$, while the contribution from $w \partial u / \partial z$ (streaming) is mostly current-related, $(\langle u><c>$ ). Finally, the advection of turbulence properties (term 2 and 3 of equation (3.4) and (3.5)), has only a marginal effect on the sediment flux profile and is not further discussed.
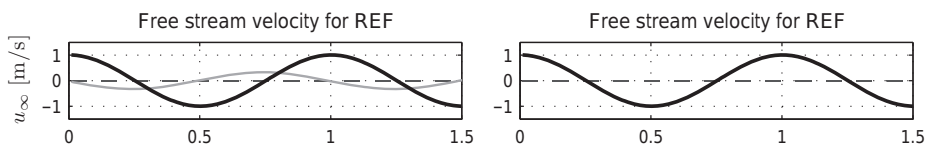

(a) $\Delta u(z, t)$ from $w \partial u / \partial z$
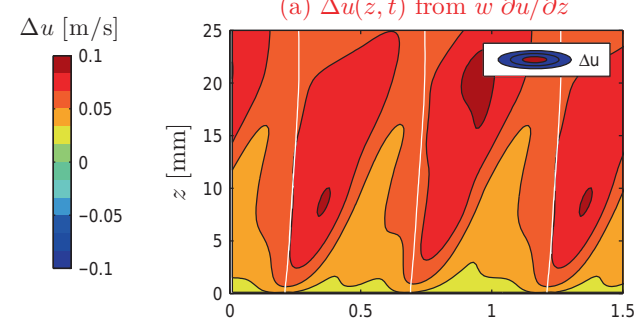

(c) $\Delta u(z, t)$ from $u \partial u / \partial x$
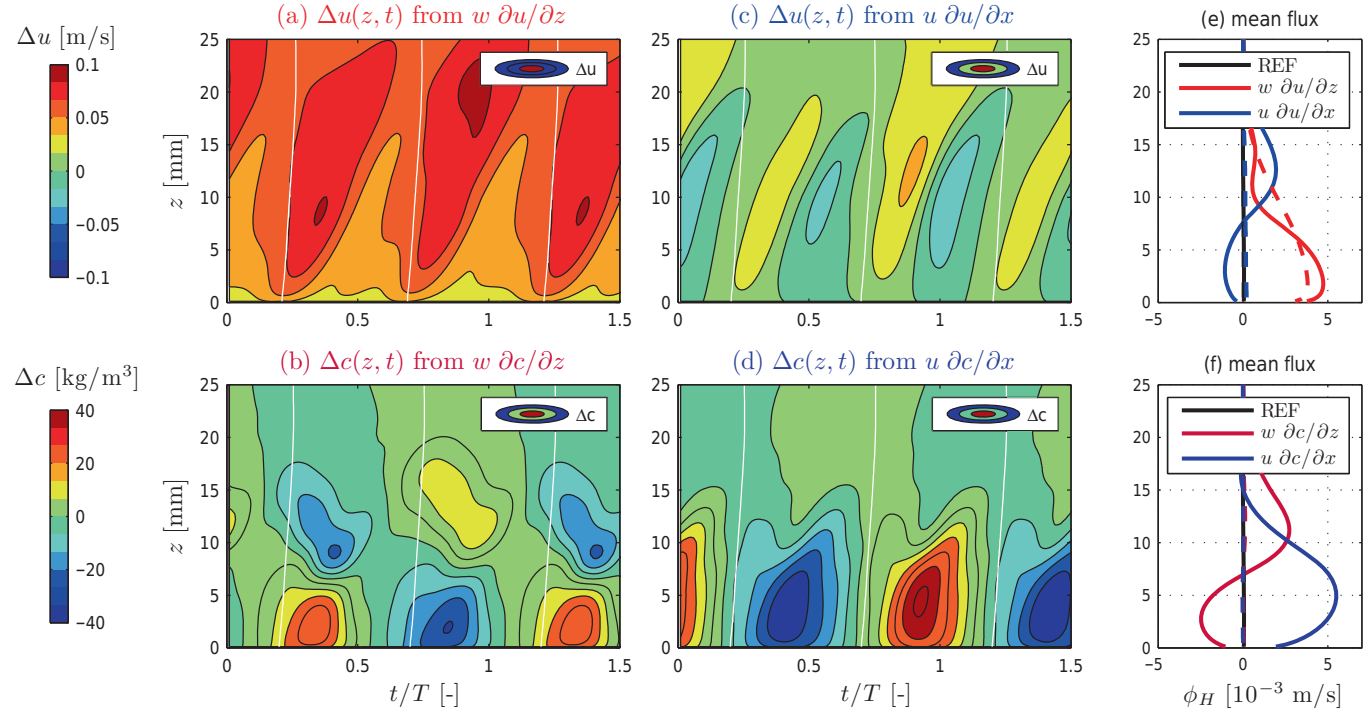

Figure 3.8: Surplus of horizontal velocity $(a, c)$ or sediment concentration $(b, d)$ induced by the various advective terms, with their consequence for the mean sediment flux (e, f). REF: reference simulation with all advective terms switched off. Solid lines: total wave averaged sediment flux $\overline{u c}$; dashed lines: current related sediment flux $\overline{u c}$. The top panels show free stream velocities. The white lines in (a-d) indicate flow reversal. Condition: sinusoidal wave with $T=6.5 \mathrm{~s}, \hat{u}_{\infty}=1.0 \mathrm{~m} / \mathrm{s}, h=3.5 \mathrm{~m}, d_{50}=0.1 \mathrm{~mm}$. 
Table 3.4: Overview of Free Surface Effects (with sediment transport values matching Figure 3.8)

\begin{tabular}{|c|c|c|c|c|c|}
\hline $\mathrm{Nr}$ & physical process & $\begin{array}{c}\text { mathematical } \\
\text { term }\end{array}$ & primary effect & $\begin{array}{c}\text { net } \\
\text { transport } \boldsymbol{q}_{\mathrm{s}} \\
{\left[10^{-6} \mathrm{~m}^{2} / \mathrm{s}\right]}\end{array}$ & $\begin{array}{c}\begin{array}{c}\text { current } \\
\text { related part }\end{array} \\
{\left[10^{-6} \mathrm{~m}^{2} / \mathrm{s}\right]}\end{array}$ \\
\hline 1 & vertical momentum advection & $w \partial u / \partial z$ & onshore streaming & 38.3 & 40.0 \\
\hline 2 & vertical sediment advection & $w \partial \mathrm{c} / \partial z$ & adapted phase-lag & 9.0 & 0.9 \\
\hline 3 & horizontal sediment advection & $u \partial c / \partial x$ & concentration modulation & 50.0 & 0.1 \\
\hline 4 & horizontal momentum advection & $u \partial u / \partial x$ & velocity skewness & 9.6 & 2.6 \\
\hline
\end{tabular}

\subsubsection{Advection processes: tests for realistic waves}

Where the effects of the various advection processes on velocities and concentrations were illustrated for sinusoidal waves in section 3.4.2, we now investigate their relevance for sediment transport for more realistic non-linear wave conditions. For that we define a number of test conditions with constant wave period $T$ and water depth $h$, but gradually increasing wave height $H$. From $T, h$ and $H$ we determine the fluctuating part of the near bed free stream horizontal velocity $\tilde{u}_{\infty}(t)$ with the Fourier approximation method of Rienecker and Fenton [1981]. This results in velocity signals with increasing velocity skewness for increasing $H$. Using the method of Rienecker and Fenton [1981], acceleration skewness from steepening of the wave towards breaking is not considered. Seaward of the surf zone, we consider this a justified approach, based on indications that waves in that region are predominantly velocity skewed [Ruessink et al., 2009]. An overview of the test conditions is given in Table 3.5. Next to wave height $H$, the table gives the amplitudes of four harmonic components of $\tilde{u}_{\infty}$, namely $\hat{u}_{\infty, 1-4}$, together with velocity skewness measures $R=\tilde{u}_{\infty, \text { crest }} /$ $\left(\tilde{u}_{\infty, \text { crest }}-\tilde{u}_{\infty, \text { trough }}\right)$ and $S k_{u}=\overline{\tilde{u}_{\infty}^{3}} /\left(\overline{\tilde{u}_{\infty}^{2}}\right)^{1.5}$, energy measure $u_{r m s}=\sqrt{\overline{\tilde{u}_{\infty}^{2}}}$ and the third order velocity moment $<\tilde{u}_{\infty}{ }^{3}>$, all determined from $\tilde{u}_{\infty}$. This free stream velocity $\tilde{u}_{\infty}$ is used to force the model; the mean velocity is allowed to develop freely (open simulation).

For the defined test cases, the sediment transport has been simulated with all advective terms switched on (FLU, because it models the flume situation), with all advective terms switched off (REF), and with only $w \partial u / \partial z, w \partial c / \partial z, u \partial c / \partial x$ or $u \partial u / \partial x$ switched on individually. This has been done for both medium sized sand $\left(\mathrm{d}_{50}=0.25 \mathrm{~mm}\right)$ and fine sized sediment $\left(\mathrm{d}_{50}=0.14 \mathrm{~mm}\right)$. The computed transport rates are shown in Figure 6.1, plotted against the third order velocity moment. For the fine grains, the percentage of the difference in transport between FLU and REF covered by a single advection term has been added to Table 3.5 , where TERM[\%] $=\left(q_{\mathrm{s}, \mathrm{TERM}}-q_{\mathrm{s}, \mathrm{REF}}\right) /\left(q_{\mathrm{s}, \mathrm{FLU}}-q_{\mathrm{s}, \mathrm{REF}}\right)$.

The computed transport rates provide insight in the relative importance of individual advective processes in explaining the differences between tunnels and flumes, and show how the relative contribution of the various terms changes with changing wave and bed conditions. We learn from Figure 3.9 that progressive wave streaming, induced by $w \partial u / \partial z$, indeed contributes substantially to onshore sediment transport. For the medium grains almost the complete difference between flume 

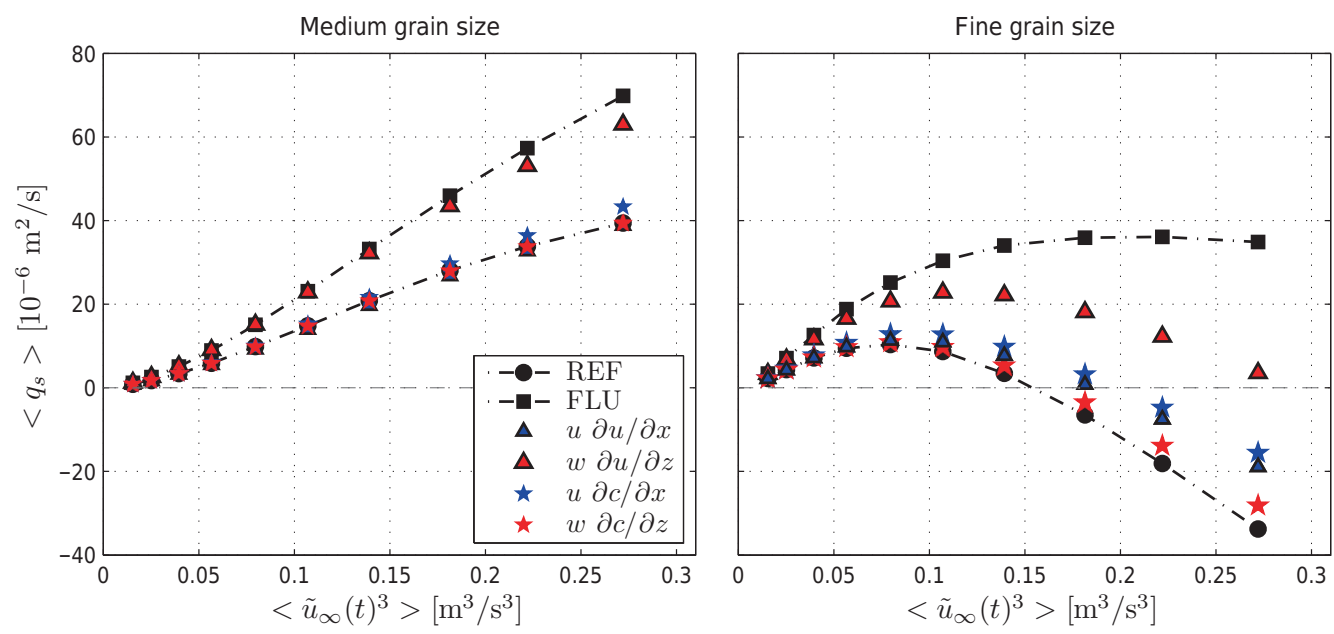

Figure 3.9: Net transport rates $\left\langle q_{\mathrm{s}}\right\rangle$ of medium $(0.25 \mathrm{~mm})$ and fine $(0.14 \mathrm{~mm})$ sized sediments for the wave conditions of Table 3.5, plotted against $\left\langle\tilde{u}_{\infty}{ }^{3}\right\rangle$. The figure shows results obtained with all advective terms switched on (FLU), all advective terms switched off (REF), and only $w \partial u / \partial z, w \partial c / \partial z, u \partial c / \partial x$ or $u \partial u / \partial x$ switched on.

Table 3.5: Overview of test conditions ${ }^{\mathrm{a}}$, with relative contribution of individual advective terms to the total sediment transport ${ }^{\mathrm{b}}$.

\begin{tabular}{|c|c|c|c|c|c|c|c|c|c|c|c|c|}
\hline $\begin{array}{c}\boldsymbol{H} \\
{[\mathrm{m}]}\end{array}$ & $\begin{array}{c}\hat{\boldsymbol{u}}_{\infty, \mathbf{1}} \\
{[\mathrm{m} / \mathrm{s}]}\end{array}$ & $\begin{array}{c}\hat{\boldsymbol{u}}_{\infty, \mathbf{2}} \\
{[\mathrm{m} / \mathrm{s}]}\end{array}$ & $\begin{array}{c}\hat{\boldsymbol{u}}_{\infty, 3} \\
{[\mathrm{~m} / \mathrm{s}]}\end{array}$ & $\begin{array}{c}\hat{\boldsymbol{u}}_{\infty, 4} \\
{[\mathrm{~m} / \mathrm{s}]}\end{array}$ & $\begin{array}{c}\boldsymbol{R} \\
{[-]}\end{array}$ & $\begin{array}{c}S \boldsymbol{k}_{\mathbf{u}} \\
{[-]}\end{array}$ & $\begin{array}{c}\boldsymbol{u}_{\mathbf{r m s}} \\
{[\mathrm{m} / \mathrm{s}]}\end{array}$ & $\begin{array}{l}<\tilde{\boldsymbol{u}}_{\infty}>^{3} \\
{\left[\mathrm{~m}^{3} / \mathrm{s}^{3}\right]}\end{array}$ & $\begin{array}{c}w \partial u / \partial z \\
{[\%]}\end{array}$ & $\begin{array}{c}w \partial \mathrm{c} / \partial z \\
{[\%]}\end{array}$ & $\begin{array}{c}u \partial c / \partial x \\
{[\%]}\end{array}$ & $\begin{array}{c}u \partial u / \partial \boldsymbol{x} \\
{[\%]}\end{array}$ \\
\hline 0.7 & 0.50 & 0.08 & 0.01 & - & 0.58 & 0.34 & 0.36 & 0.016 & 96 & 0 & 5 & -4 \\
\hline 0.8 & 0.56 & 0.10 & 0.01 & - & 0.59 & 0.38 & 0.41 & 0.025 & 90 & 1 & 7 & -3 \\
\hline 0.9 & 0.62 & 0.13 & 0.02 & - & 0.60 & 0.43 & 0.45 & 0.040 & 83 & 2 & 11 & -0 \\
\hline 1.0 & 0.68 & 0.15 & 0.02 & - & 0.61 & 0.47 & 0.50 & 0.057 & 76 & 3 & 14 & 3 \\
\hline 1.1 & 0.74 & 0.18 & 0.03 & 0.01 & 0.62 & 0.51 & 0.54 & 0.080 & 70 & 4 & 16 & 7 \\
\hline 1.2 & 0.79 & 0.21 & 0.04 & 0.01 & 0.63 & 0.55 & 0.58 & 0.107 & 65 & 5 & 19 & 10 \\
\hline 1.3 & 0.84 & 0.24 & 0.05 & 0.01 & 0.64 & 0.59 & 0.62 & 0.139 & 61 & 6 & 21 & 14 \\
\hline 1.4 & 0.89 & 0.27 & 0.06 & 0.01 & 0.65 & 0.63 & 0.66 & 0.182 & 58 & 7 & 23 & 17 \\
\hline 1.5 & 0.93 & 0.30 & 0.07 & 0.01 & 0.65 & 0.67 & 0.69 & 0.222 & 56 & 8 & 25 & 20 \\
\hline 1.6 & 0.97 & 0.33 & 0.08 & 0.01 & 0.66 & 0.71 & 0.73 & 0.272 & 54 & 8 & 27 & 22 \\
\hline
\end{tabular}

a) $T=6.5 \mathrm{~s}$ and $h=3.5 \mathrm{~m}$ in all tests

b) for the fine sand tests, with $d_{50}=0.14 \mathrm{~mm}$ 
(FLU) and tunnel (REF) simulations is covered with vertical momentum advection taken into account. However, in case of fine sand, with higher volumes of sediment in suspension, also the gradients in horizontal advection become important, especially $u \partial c / \partial x$. Table 3.5 shows that the relative contribution of this term also increases with increasing wave height. For the wave and bed conditions from the realistic ranges investigated here, the effect of $w \partial c / \partial z$ turns out to be negligible. Finally, note that the sum of the four separate contributions is smaller than but close to $100 \%$ for the least energetic and just over $100 \%$ for the most energetic condition. This means that the interaction between the various advective processes is small.

\subsection{DISCUSSION}

\subsubsection{Relevance for sediment transport formulas}

We have shown that both progressive wave streaming and gradients in horizontal advection are free surface effects that can contribute significantly to sediment transport beneath waves. Therefore we believe that these free surface effects should be accounted for in sediment transport formulas. This is generally not the case in transport formulas used in present day morphodynamic modeling, developed and calibrated from tunnel experiments (see e.g Davies et al. [2002]). Sediment transport formulas predict the transport from the free stream velocity or bed shear stress. 'Quasi-steady' formulas directly relate the instantaneous transport to the instantaneous velocity or stress through power laws and empirical coefficients (e.g. Bailard [1981], Ribberink [1998]). 'Semi-unsteady' formulas account for phase-lag effects through inclusion of a phase-lag parameter representing the ratio of sediment settling time and wave period (e.g. Dibajnia and Watanabe [1998], DohmenJanssen et al. [2002]). The first to account for progressive wave streaming in transport formulas were Nielsen [2006] and Van Rijn [2007]. They compute the transport with either an extra onshore wave-averaged (free stream) velocity [Van Rijn, 2007] or bed shear stress [Nielsen, 2006] added to the oscillatory input of their transport formula. Note that new parameterizations for this additional mean velocity and stress are provided by Kranenburg et al. [2012]. The effect of horizontal (sediment) advection gradients was not included, or it was assumed to be strongly correlated to the streaming effect [Nielsen, 2006]. This study's differentiation between the various free surface effects shows that the relative contribution is strongly grain size dependent. Here we present a parameterization for the horizontal advection effects consistent with the insights from this study.

Firstly, consider a simple transport formula that expresses the depth integrated sediment flux $q_{\mathrm{s}}$ as function of the free stream velocity $u_{\infty}$ and the depth-averaged volume concentration $C(t)$ :

$$
q_{s}(t)=\int_{z=z b e d}^{z b e d+\delta} u c d z=\alpha \delta u_{\infty}(t) C(t)
$$

with $\delta$ the thickness of the layer over which transport (and averaging) takes place and $\alpha$ a distribution coefficient related to the shape of the concentration and velocity profiles $(\mathrm{O}(1))$. Secondly, note that the time-dependent behavior of the depth-averaged concentration $C(t)$ in gradually-varying flows can be represented in a schematic way by a relaxation equation: 


$$
\frac{\partial C(t)}{\partial t}=\frac{\gamma\left\{C_{e q}(t)-C(t)\right\}}{T_{a}}
$$

(see Galappatti and Vreugdenhil [1985]). In this relaxation equation, $T_{\mathrm{a}}$ is the time scale of adaptation of the sediment concentration to the equilibrium concentration $C_{\text {eq }}$, and $\gamma$ is a coefficient related to the shape of the concentration profile. The (depth-averaged) $C_{\text {eq }}$ reflects the 'carrying capacity' of the flow: the concentration for which the sediment settling and pick-up are equal. $C_{\mathrm{eq}}$ is directly related to the instantaneous forcing through the Shields number $\theta$ (see e.g. Van Rijn [1993]). Here, we apply $C_{\mathrm{eq}}(t)=\beta \theta(t)$, with $\beta$ a coefficient. The key element of the parameterization is the expression for $T_{\mathrm{a}}$. Starting from the advection-diffusion equation, we derive in appendix $\mathrm{E}$ that the advection effects in horizontally non-uniform flow can be included in the concentration equation (3.20) and transport formula (3.19) with:

$$
T_{a}(t)=\frac{\delta}{w_{s}}\left\{1-\frac{\alpha u_{\infty}(t)}{c_{p}}\right\}
$$

Here $c_{\mathrm{p}}$ is the wave propagation speed and $\left\{1-\alpha u_{\infty} / c_{\mathrm{p}}\right\}$ is $<1$ during onshore flow and $>1$ during offshore flow. Note that in oscillatory flows, $T_{\mathrm{a}}$ reduces to $\delta / w_{\mathrm{s}}$. This is the settling time used also by Dohmen-Janssen et al. [2002] in the phase-lag parameter $T_{\mathrm{a}} / T$ for the semi-unsteady description of fine sand transport in tunnels. Hereby $\delta$ is the particle entrainment height (also an appropriate measure for the transport layer thickness), and $w_{\mathrm{s}}$ is the settling velocity. Next, for medium to coarse sand, $\delta / w_{\mathrm{s}}$ will be small. In that case, equation (3.20) yields concentrations immediately adapting to changes in the forcing, and sediment transport formula (3.19) becomes quasi-steady. With the full equation for $T_{\mathrm{a}}$, the main features of the advection effects under progressive waves are represented: (1) the concentration will adapt faster during the onshore motion than during the offshore motion, (2) increased/decreased maximum concentration will be found under the wave crest/trough, and (3) the advection effects will increase with decreasing grain size.

We illustrate the behavior of the parameterization with Figure 3.10. Figure 3.10(a) shows the concentration beneath a sinusoidal wave computed from relaxation equation (3.20) respectively with a quasi-steady approach $\left(T_{\mathrm{a}}=0\right)$, with phase-lag effects $\left(T_{\mathrm{a}}=\delta / w_{\mathrm{s}}\right)$, and with phase-lag effects beneath progressive waves, i.e. with $T_{\mathrm{a}}$ from equation (3.21). Comparison with Figure 3.8(d) shows that the latter yields concentration behavior consistent with the numerical model results. Next, Figure 3.10(b) shows, for the cases of Table 3.5, that also the numerically computed $\left\langle q_{\mathrm{s}}\right\rangle$ can be reproduced well using equation (3.19), (3.20) and (3.21). In these calculations, we set the transport layer thickness to 10 times the sheet-flow layer thickness: $\delta=10 \delta_{\mathrm{s}}$. From Dohmen-Janssen et al. [2002], we use $\delta_{\mathrm{s}}=35 d_{50} \theta_{\max }$. The maximum Shields parameter $\theta_{\max }=1 / 2 f_{\mathrm{w}} u_{\max }{ }^{2} /\left(\Delta g d_{50}\right)$. We computed $f_{\mathrm{w}}$ following Swart [1974] with bed roughness height $k_{\mathrm{N}}=2 d_{50}$. Settling velocity $w_{\mathrm{s}}$ is computed from equation (3.9)b. Coefficients $\alpha, \beta$ and $\gamma$ were used as calibration parameters tuning the balance between the processes. Note that the effects of horizontal sediment and momentum advection are strongly correlated (Figure 6.1; Table 3.5). Therefore parameter $T_{\mathrm{a}}$ can be applied to account for both advection processes together. 

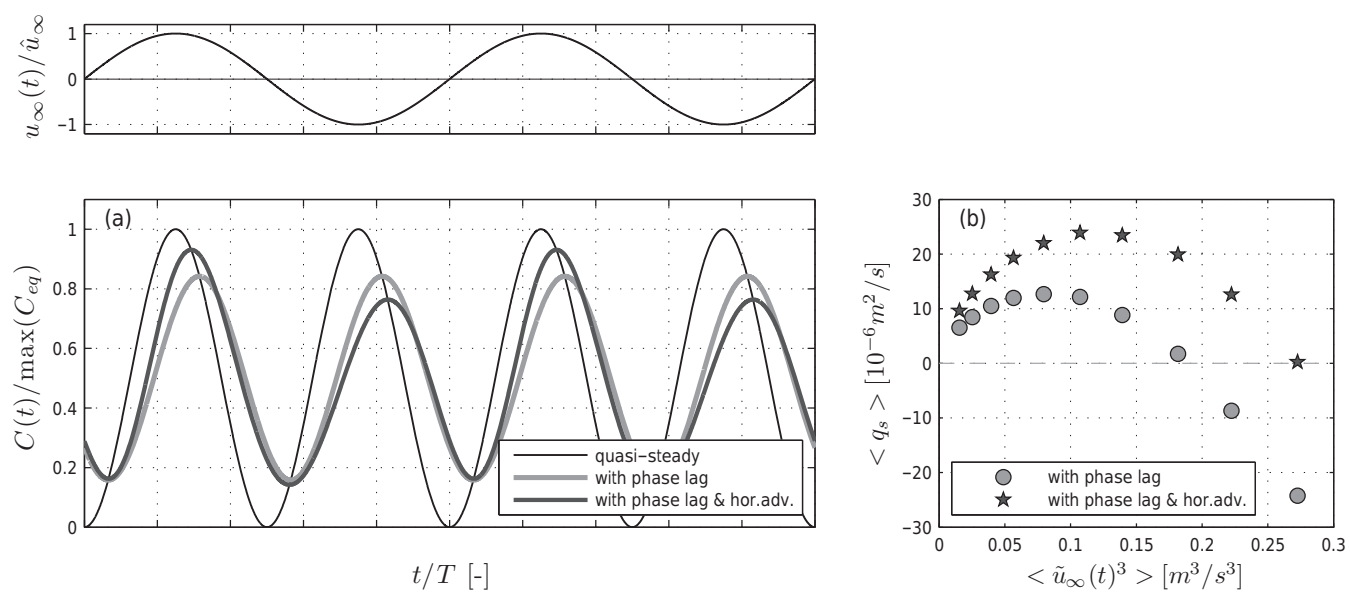

Figure 3.10: (a) Depth-averaged concentration $C$ beneath a sinusoidal wave (upper panel) respectively with a quasi-steady approach (thin black line), with phase-lag effects after Dohmen-Janssen et al. [2002] (thick light gray line), and with phase-lag in combination with horizontal advection effects (dark gray line, $T_{\mathrm{a}}$ according equation (3.21)); (b) Period-averaged sediment transport $\left\langle q_{\mathrm{s}}\right\rangle$ for the cases of Table 3.5 computed using $T_{\mathrm{a}}$ both with and without effects of horizontal advection. Parameters case (a): $T=6.5 \mathrm{~s}, \hat{u}_{\infty}=1.2 \mathrm{~m} / \mathrm{s}, h=3.0 \mathrm{~m}$, $d_{50}=0.14 \mathrm{~mm}$; used coefficients: $\alpha=2.0, \beta=0.01, \gamma=10$;

Considering the flume measurements of transport of fine sand under velocity-skewed waves (Figure 3.5 , panel b), one may wonder whether there is any need to let transport formulas evolve further away from the simple quasi-steady approach. After all, the correlation between $\left\langle q_{\mathrm{s}}\right\rangle$ and $\left\langle u_{\text {red }}{ }^{3}\right\rangle$ for these cases is very strong. One should realize that in these cases, the offshore transport from phaselag effects, so much important in velocity-skewed oscillatory tunnel flow over fine sand, and the onshore transport from advection effects nearly completely cancel each other out. These processes will not always (counter)act in the same balance. For instance when a velocity-skewed wave becomes steeper, the onshore contribution from advection effects remains, while the offshore contribution due to phase-lag effects decreases. (For purely acceleration skewed waves, phase-lag effects even contribute to onshore transport [Van $\operatorname{der} A, 2010]$ ). We therefore believe that both processes should be considered in parameterized transport formulas.

\subsubsection{Limitations of this study}

Both in the model formulation and validation, this study is limited to the suspension-mode sheetflow regime. The numerical tests to capture the various advection effects were carried out for a parameter range extending beyond this regime. Herein, we neglect that actually ripples may be expected beneath the lowest energy waves of Table 3.5 (Shields number $\theta<0.8$ ). The effects of streaming and horizontal advection on net transport rates over rippled beds, with more complicated flow patterns, are still unknown and need further research. Other issues not considered in the present study are the relevance for sediment transport of bed level variation and spreading in grain size. The 
potential role of the sieve curve width for the transport rates observed by Schretlen [2012] may initiate further research here on.

\subsection{CONCLUSIONS}

A numerical model has been developed to investigate the influence of free surface effects on transport of sediment in the wave boundary layer beneath regular progressive waves. The 1DV Reynolds Averaged Navier-Stokes boundary layer model with an advection-diffusion formulation for sediment concentration and a $k-\varepsilon$ turbulence closure with feedback of sediment on the flow through stratification effects has been successfully validated with recent full scale flume measurements on both boundary layer flow and suspension mode sheet-flow sediment transport under velocity-skewed waves. The validation showed that progressive wave streaming and stratification effects are essential processes to reproduce measured wave-averaged current profiles. As with the transport measurements, the model results show a reversal from off- to onshore waveaveraged transport of fine sediment under influence of the free surface effects. It was subsequently investigated to what extent the increased onshore transport could be attributed to progressive wave streaming. We conclude that this onshore streaming indeed contributes largely to increased onshore transport rates in flumes compared to tunnels. However, especially for fine grains, also other free surface effects are important. In particular gradients in horizontal advection of sediment in the nonuniform flow field beneath surface waves are found to influence fine sand transport significantly. This mechanism amplifies respectively reduces the maximum concentration during onshore respectively offshore motion, causing increased onshore transport rates. Therefore we conclude that, next to streaming, also the latter should be considered in formulas of wave-induced sediment transport in morphodynamic modeling. It is proposed to incorporate this process through a phase-lag parameter with a wave-phase dependent adaptation time $T_{\mathrm{a}}$ for sediment concentration in unsteady flow. The proposed parameter $T_{\mathrm{a}}$, given in equation (3.21), covers the relevant characteristics of the physical process, yields transport rates comparable to the numerical model and is therefore a suitable parameterization to be included in practical sand transport formulas. 


\title{
4 SHEET-FLOW BENEATH WAVES: EROSION DEPTHS AND SEDIMENT FLUXES AND THEIR DEPENDENCE ON GRAIN SIZE AND STREAMING ${ }^{3}$
}

\begin{abstract}
We study erosion depth and sediment fluxes for wave-induced sheet-flow, and their dependency on grain size and streaming. Hereto, we adopt a continuous two-phase model describing the motion of water and sediment. To make the model applicable to the range of sediment sizes of our interest, it appears necessary to adapt the fluid turbulence closure of the model. Good reproductions of measured erosion depth of fine, medium and coarse sized sand beds are obtained with adapted formulations for grain - carrier flow turbulence interaction. Also concentration and velocity profiles at various phases of the wave are reproduced well by the model, although inaccuracies stay present for fine sand simulations, especially around flow reversal and subsequent flow acceleration. Comparison of sediment flux profile from simulations for horizontally uniform oscillatory flow as in flow tunnels and horizontally non-uniform flow as under free surface waves, shows that especially for fine sand onshore fluxes inside the sheet-flow layer increase under influence of progressive wave effects. This includes both the current-related and the wave-related contribution to the periodaveraged sheet-flow sediment flux. The simulation results are consistent with trends for fine and medium sized sediment flux profiles observed from tunnel and flume experiments. Our exploration shows that this two-phase model can become a valuable instrument for further study and parameterization of sheet-flow layer processes.
\end{abstract}

\footnotetext{
${ }^{3}$ This chapter is the result of a collaboration with dr. T.J. Hsu, University of Delaware, Newark, DE, USA
} 


\subsection{INTRODUCTION}

Under high waves sand in the near-shore zone is transported as sheet-flow. The main characteristics of this phenomenon are that bed forms are washed away and that the motion of sediment extends down to several grain diameters below the initial bed level (erosion depth). This moving layer with high concentrations of sediment (sheet-flow layer) is held responsible for the larger part of the sediment transport. Good predictions of wave-induced sediment transport rates are of utmost importance for coastal engineering work. Therefore, it is relevant to develop detailed insights in sheet-flow mechanisms and characteristics and to develop tools to quantify transport rates in the sheet-flow regime.

Usually, morphodynamic models make use of (semi-)empirical sediment transport formulas. These formulas are generally based on sets of experiments with a limited number of wave and bed conditions. Next, most of these experiments have been carried out in oscillating flow tunnels, while it has become clear from recent flume experiments that free surface effects not included in these tunnel experiments can largely affect the transport rates and underlying processes . More detailed numerical models can be helpful to investigate parameter values that have not been investigated experimentally and to improve the insight in the underlying processes. Parameterization of the numerical model results can be helpful to improve the physical basis of these transport formulas.

Various types of numerical models are available. Here we mention (quasi) single phase and continuous two-phase wave boundary layer models. Models of the first type have been very helpful to investigate the role of wave shape , [Holmedal and Myrhaug, 2006], [Ruessink et al., 2009], grain size [Hassan and Ribberink, 2010], stratification [Conley et al., 2008] and free surface effects [Holmedal and Myrhaug, 2009], [Kranenburg et al., 2012], [Kranenburg et al., 2013] on boundary layer flow and/or sediment transport and have been applied to predict bar migration [Henderson et al., 2004] [Hsu et al., 2006]. In these single phase models, particles are assumed to move with the fluid velocity (apart from the settling velocity), and sediment concentrations are determined from an advection-diffusion equation for the concentration with a fixed-level lower boundary condition that relates the near-bed concentration or vertical sediment flux to the local shear stress through an empirical reference concentration or pick-up function. This means that these models actually do not solve the details of the sheet-flow layer, like the fluctuating position of the immobile bed and adapted flow and sediment dynamics in the region of high sediment concentrations.

Alternatively, sheet-flow models have been developed based on theory for continuous two-phase flow. These models describe the motion of water and sediment from the immobile bed into the suspension layer with separate momentum equations and mutual interactions between the phases. In principle, this makes it possible to simulate sediment suspension processes without empirical parameterizations for sediment pick-up and without any need to distinguish between bed load and suspended load. Examples of this type of models are [Asano, 1990], [Dong and Zhang, 1999], [Hsu et al., 2004], [Li et al., 2008], [Yu et al., 2010]). Most important differences between the various 
two-phase models appear in the closures of respectively the turbulent stresses and interparticle stresses. Regarding the first, a distinction can be made between models with mixing length, oneequation and two-equation turbulence closures. Interparticle stresses are modeled with rheological equations like Bagnold's expressions for the viscous \& inertia regime ([Bagnold, 1954], see also [Ahilan and Sleath, 1987]), or using the concept of 'granular temperature' from collisional granular flow theory for the energy of the particle fluctuations [Jenkins and Hanes, 1998]. Next, differences are found in the modeling of the particle-fluid interaction on the level of momentum equations (e.g. different descriptions of the drag force, omission of the added mass force) and in the modeling of particle influence on the carrier flow turbulence. Finally, so far only Yu et al. [2010] consider free surface effects by including horizontal and vertical advection of fluid and sediment momentum and energy.

From the aspiration to improve sediment transport formulas in morphodynamic modeling, it is our objective to investigate sheet-flow layer behavior and how this relates to sediment transport. We are especially interested in erosion depth, sheet-flow layer thickness and the distribution of the sediment flux over the vertical profile, and differences therein under influence of sand grain size variation and free surface effects, such as the onshore 'progressive wave streaming' in the boundary layer generated by the vertical orbital motion [Longuet-Higgins, 1958]. Parameterizations of these characteristics will be very useful for practical sand transport formulas.

Hereto, this study explores the possibility to predict erosion depth, sheet-flow layer thickness and sediment fluxes, and trends in their dependency on streaming and grain size variation, using a twophase continuum approach. Hereby, the model of Yu et al. [2010] is used as starting-point, because this is the only two-phase model that considers horizontally non-uniform flow (as occurs under progressive waves). Firstly, the background of the model is described in section 2. Secondly, an inventory is given of the data available for model validation (section 3). Section 4 describes validation tests on erosion behavior for various grain sizes resulting in model adaptations improving the grain size dependent model behavior. Next, section 5 describes model-data comparison on timedependent concentration profiles and time-dependent and wave-averaged velocity profiles, the latter both with and without progressive wave streaming. The reproduction quality is discussed and a sensitivity analysis is carried out to investigate how further improvement of the model performance could be achieved. Subsequently, in section 6 the model is applied to investigate trends in sediment flux profiles for fine and medium sized sand both without and with progressive wave streaming. Finally, section 7 provides a discussion and section 8 summarizes the conclusions.

\subsection{MODEL FORMULATION}

\subsubsection{Model background}

The two-phase model we adopt here has been developed originally by Hsu et al. [2003] for dilute sediment transport in steady and oscillatory flow. It has subsequently been extended with interparticle stress formulations to model sheet-flow of massive particles [Hsu et al., 2004]. Amoudry et 
al. [2008] have applied the model to sheet-flow of coarse and medium sized sand. The model applicability has been extended by $Y u$ et al. [2010] from horizontally uniform flow as present in oscillatory flow tunnels to horizontally non-uniform flow as present under propagating waves.

The model can be classified as a 1 dimensional vertical (1DV) two-phase model with a two-equation $(\mathrm{k}-\varepsilon)$ fluid turbulence and an interparticle stress closure using the 'granular temperature' concept. The turbulence averaged momentum equations have been derived using Favre-averaging. In Favreaveraging, ensemble-averaging is applied to the momentum per unit mass of each phase instead of the velocity. This avoids the need to account for correlations between concentration and velocity fluctuations in the continuity equation. The horizontal non-uniformity has been accounted for within the $1 \mathrm{DV}$ approach by the transformation:

$$
\frac{\partial}{\partial x}=-\frac{1}{c} \frac{\partial}{\partial t}
$$

which assumes that the waves propagate (with $c$ the propagation velocity) without changing their form. Below, the model equations are given in the averaged and transformed form, as solved by the numerical model.

\subsubsection{Governing equations}

The continuity equations for the fluid $(f)$ and sediment $(s)$ phase are:

$$
-\frac{1}{c} \frac{\partial(1-\phi) u^{f}}{\partial t}+\frac{\partial(1-\phi) w^{f}}{\partial z}-\frac{\partial \phi}{\partial t}=0
$$

and

$$
-\frac{1}{c} \frac{\partial \phi u^{s}}{\partial t}+\frac{\partial \phi w^{s}}{\partial z}+\frac{\partial \phi}{\partial t}=0
$$

with $\phi$ the volumetric concentration of sediment and $u$ and $w$ the (Favre-averaged) velocity components in horizontal $(x)$ respectively vertical $(z)$ direction. The momentum equations of the fluid phase in the $x$ - and $z$-directions can respectively be written as:

$$
\left(1-\frac{u^{f}}{c}\right) \frac{\partial u^{f}}{\partial t}+w^{f} \frac{\partial u^{f}}{\partial z}=-\frac{1}{\rho^{f}} \frac{\partial P^{f}}{\partial x}+\frac{1}{\rho^{f}(1-\phi)} \frac{\partial \tau_{x z}^{f}}{\partial z}-\frac{\beta \phi}{\rho^{f}(1-\phi)}\left(u^{f}-u^{s}\right)-\frac{\beta v_{f t}}{c \rho^{f}(1-\phi) \sigma_{c}} \frac{\partial \phi}{\partial t}
$$

and

$$
\frac{1}{\rho^{f}} \frac{\partial P^{f}}{\partial z}=-\left(1-\frac{u^{f}}{c}\right) \frac{\partial w^{f}}{\partial t}-w^{f} \frac{\partial w^{f}}{\partial z}+\frac{1}{\rho^{f}(1-\phi)} \frac{\partial \tau_{z z}^{f}}{\partial z}+g-\frac{\beta \phi}{\rho^{f}(1-\phi)}\left(w^{f}-w^{s}\right)+\frac{\beta v_{f t}}{\rho^{f}(1-\phi) \sigma_{c}} \frac{\partial \phi}{\partial z}
$$


with $P^{f}$ the fluid pressure, $g$ the gravitational acceleration, $\rho^{f}$ the fluid density, and $\tau_{x z}^{f}$ and $\tau_{z z}^{f}$ the shear and normal stresses of the fluid phase, including both the viscous and turbulent stresses, the latter modeled using the Boussinesq hypothesis. The last two terms of both equations originate from interface momentum transfer by drag. Hereby, the second term appears as a result of the ensembleaveraging: parallel to the Boussinesq hypothesis, the correlation between concentration and velocity fluctuations is modeled using a gradient transport [McTigue, 1981], with $v_{f t}$ the eddy viscosity and $\sigma_{\mathrm{c}}$ the Prandtl-Schmidt number (see appendix F for an elaboration hereof). The closure for $v_{f t}$ is discussed below. The drag parameter $\beta\left[\mathrm{kg} / \mathrm{m}^{3} / \mathrm{s}\right]$ is a function of particle diameter $d$, fluid density $\rho^{f}$, relative velocity magnitude $U_{r}=\left|\vec{u}^{f}-\vec{u}^{s}\right|$ and particle Reynolds number $\operatorname{Re}_{p}=U_{r} d / v_{f}$ :

$$
\beta=\frac{\rho^{f} U_{r}}{d}\left(\frac{18}{\operatorname{Re}_{\mathrm{p}}}+0.3\right) \frac{1}{(1-\phi)^{n}}
$$

A concentration dependent correction factor $(1-\phi)^{-n}$ is added to account for increased drag under influence of surrounding particles [Richardson and Zaki, 1954]. Herein, the coefficient $n$ depends on $\phi$ and $R e_{\mathrm{p}}$ and is computed following Fredsøe and Deigaard [1992] (p.200). In the model, the vertical fluid velocity is solved from the fluid continuity equation. The fluid momentum equation in $z$-direction is used to determine the vertical pressure gradient, needed to solve the sediment motion.

The momentum equations of sediment phase in the $x$ - and $z$-directions are respectively

$$
\left(1-\frac{u^{s}}{c}\right) \frac{\partial \phi u^{s}}{\partial t}-\frac{\phi u^{s}}{c} \frac{\partial u^{s}}{\partial t}+\frac{\partial \phi u^{s} w^{s}}{\partial z}=-\frac{\phi}{\rho^{s}} \frac{\partial P^{f}}{\partial x}+\frac{1}{\rho^{s}} \frac{\partial \tau_{x z}^{s}}{\partial z}+\frac{\beta \phi}{\rho^{s}}\left(u^{f}-u^{s}\right)+\frac{\beta v_{f i}}{c \rho^{s} \sigma_{c}} \frac{\partial \phi}{\partial t}
$$

and

$$
\left(1-\frac{u^{s}}{c}\right) \frac{\partial \phi w^{s}}{\partial t}-\frac{\phi w^{s}}{c} \frac{\partial u^{s}}{\partial t}+\frac{\partial \varphi w^{s} w^{s}}{\partial z}=-\frac{\phi}{\rho^{s}} \frac{\partial P^{f}}{\partial z}+\phi g+\frac{1}{\rho^{s}} \frac{\partial \tau_{z z}^{s}}{\partial z}+\frac{\beta \phi}{\rho^{s}}\left(w^{f}-w^{s}\right)-\frac{\beta v_{f t}}{\rho^{s} \sigma_{c}} \frac{\partial \phi}{\partial z}
$$

with $\rho^{s}$ the sediment density and $\tau_{x z}^{s}$ and $\tau_{z z}^{s}$ the shear and normal sediment stresses, discussed later.

Table 4.1: Coefficients turbulence model

\begin{tabular}{lll}
\hline Symbol & Meaning & Value \\
\hline$\sigma_{\mathrm{t}}$ & Prandtl-Schmidt number & 0.7 \\
$\sigma_{\mathrm{k}}, \sigma_{\varepsilon}, c_{\mu} \mathrm{c}_{1 \varepsilon}, c_{2 \varepsilon}$ & Coefficients in turbulence model & $1.0 ; 1.3 ; 0.09 ; 1.44 ; 1.92$ \\
\hline
\end{tabular}




\subsubsection{Closures for the fluid and particle stresses}

The fluid stresses are modeled using the Boussinesq hypothesis, with the eddy viscosity defined as:

$$
v_{f t}=C_{\mu} \frac{(1-\phi) k_{f}^{2}}{\varepsilon_{f}}
$$

The two-equation turbulence model to compute the fluid phase turbulent kinetic energy $k_{f}$ and the turbulent dissipation rate $\varepsilon_{f}$ is described with the transport equations:

$$
\begin{aligned}
\left(1-\frac{u^{f}}{c}\right) \frac{\partial k_{f}}{\partial t}+w^{f} \frac{\partial k_{f}}{\partial z} & =\frac{1}{\rho^{f}(1-\phi)}\left(\tau_{x z}^{f t} \frac{\partial u^{f}}{\partial z}+\tau_{z z}^{f t} \frac{\partial w^{f}}{\partial z}\right)+\frac{1}{(1-\phi)} \frac{\partial}{\partial z}\left[\left(v+\frac{v_{f t}}{\sigma_{k}}\right) \frac{\partial(1-\phi) k_{f}}{\partial z}\right] \\
& -\varepsilon_{f}+\frac{(s-1) g v_{f t}}{(1-\phi) \sigma_{c}} \frac{\partial \phi}{\partial z}-\frac{1}{\rho^{f}(1-\phi)} \phi \beta(1-\alpha) 2 k_{f}
\end{aligned}
$$

and

$$
\begin{aligned}
\left(1-\frac{u^{f}}{c}\right) \frac{\partial \varepsilon_{f}}{\partial t}+w^{f} \frac{\partial \varepsilon_{f}}{\partial z} & =\frac{C_{\varepsilon 1} \varepsilon_{f}}{k_{f}} \frac{1}{\rho^{f}(1-\phi)}\left(\tau_{x z}^{f t} \frac{\partial u^{f}}{\partial z}+\tau_{z z}^{f t} \frac{\partial w^{f}}{\partial z}\right)+\frac{1}{(1-\phi)} \frac{\partial}{\partial z}\left[\left(v+\frac{v_{f t}}{\sigma_{\varepsilon}}\right) \frac{\partial(1-\phi) \varepsilon_{f}}{\partial z}\right] \\
& -\frac{C_{\varepsilon 2} \varepsilon_{f}}{k_{f}} \varepsilon_{f}+\frac{C_{\varepsilon 3} \varepsilon_{f}}{k_{f}} \frac{(s-1) g v_{f t}}{(1-\phi) \sigma_{c}} \frac{\partial \phi}{\partial z}-C_{\varepsilon 3} \frac{1}{\rho^{f}(1-\phi)} \phi \beta(1-\alpha) 2 \varepsilon_{f}
\end{aligned}
$$

with specific density $s=\rho^{s} / \rho^{\mathrm{f}}$. The first three terms on the right-hand side of the transport equations describe respectively production, diffusion and dissipation. The last two terms describe modifications to the standard $k-\varepsilon$ model due to interactions between the sediment and the fluid flow turbulence [Drew, 1976], [Elghobashi and Abou-Arab, 1983]. The fourth term can be seen as the attenuation of the growth of eddies by density stratification. The fifth term models the drag-effect on the carrier flow turbulence of sediment particles that cannot completely follow the turbulent fluid velocity fluctuations. In this term, $\alpha$ is a parameter to characterize the degree the particles follow the fluid fluctuation, with a value between 0 and $1 . \alpha=1$ denotes completely passive particles, yielding no drag-induced turbulence damping. As background to the presented model formulations, appendix $\mathrm{F}$ discusses the derivation of the drag terms in the momentum and energy equations.

The question how to include the effect of sediment on the carrier flow turbulence is answered in different ways in literature. Some authors propose modifications of the turbulence model coefficients. Amoudry et al. [2008] e.g. adopted a particle concentration and particle inertia dependent $C_{\varepsilon 2}$. In this study, the model is applied with the standard (clear fluid) values for $C_{\mu}, C_{\varepsilon 1}$, $C_{\varepsilon 2}, \sigma_{\mathrm{k}}$ and $\sigma_{\varepsilon}$ (see Table 4.1), and we strive after modeling the sediment effect entirely through the description of the physical interaction mechanisms of buoyancy and drag and coefficients therein. Following Yu et al. [2010], default settings for the sediment related coefficients are $C_{\varepsilon 3}=1.2$ (based on research by Elghobashi and Abou-Arab [1983] on sediment laden jets) and 


$$
\alpha=\frac{1}{\left(1+T_{p} / T_{t}\right)}
$$

based on Rundqvist et al. [2005]. Herein, the parameters $T_{t}$ and $T_{p}$, respectively

$$
T_{t}=\frac{1}{6} \frac{k_{f}}{\varepsilon_{f}} \quad \& \quad T_{p}=\frac{\rho_{s}}{\beta}
$$

denote the time scale of the fluid turbulence and the particle response time. The latter is a measure of the time to accelerate a single particle from rest to the velocity of the surrounding fluid by drag. A relative small $T_{\mathrm{p}}$ yields large correlation between particle and fluid fluctuation $(\alpha \rightarrow 1)$. Finally, following examples for density stratified flow, the buoyancy term in the epsilon equation is switched off for stable stratifications (consistent with the single phase model of chapter 3 ). We will return to the grain - fluid turbulence interaction related model parameters in section 4 and 5 .

The sediment (particle) stresses result from interparticle interactions. The way particles interact differs throughout the water column. For the closure of the sediment stresses, various regions have been discerned [Hanes and Inman, 1985], [Zhang and Campbell, 1992], for which different descriptions of the particle stress are needed. High in the water column, the concentration of suspended sediment is very low (dilute region). In that region, particle-particle interactions can be neglected and the particle suspension is supported by turbulent mixing only. In the region below, the particles move independent of each other, but transfer momentum trough binary collisions (collisional region). Between the immobile bed and the collisional region, a transition region is present where the grains are able to move, but stay in contact with several other grains (quasi-static regime of enduring contact). This is typically the case when the sediment volume concentration $\phi$ is between the random close-packing and random loose-packing value, i.e. between 0.635 and 0.57 . In this regime, there is transfer of momentum through friction and normal stress - like in a solid while the material is moving like fluid. See Figure 4.1 for a schematization.

In the collisional regime, the behavior of the grains is modeled using the kinetic theory of granular flow. This theory is based on kinetic theory of gases describing the behavior of molecules, extended to account for slightly inelastic collisions and interstitial fluid. Key elements are a constitutive relation for moving identical, frictionless, slightly inelastic, colliding spherical particles and a transport equation for the energy of the particle velocity fluctuations or 'granular temperature' $\Theta$, respectively

$$
\tau_{i j}^{s}=\left(-p_{s}+\xi_{s} \frac{\partial u_{k}^{s}}{\partial x_{k}}\right) \delta_{i j}+\mu_{s}\left(\frac{\partial u_{i}^{s}}{\partial x_{j}}+\frac{\partial u_{j}^{s}}{\partial x_{i}}\right)
$$

and

$\rho^{s}\left[\left(1-\frac{u^{s}}{c}\right) \frac{\partial \phi \Theta}{\partial t}-\frac{\phi \Theta}{c} \frac{\partial u^{s}}{\partial t}+\frac{\partial \phi \Theta w^{s}}{\partial z}\right]=\tau_{x z}^{s} \frac{\partial u^{s}}{\partial z}+\tau_{z z}^{s} \frac{\partial w^{s}}{\partial z}-\frac{\partial Q}{\partial z}-\gamma+2 \beta \phi\left(\alpha k_{f}-\Theta\right)$ 


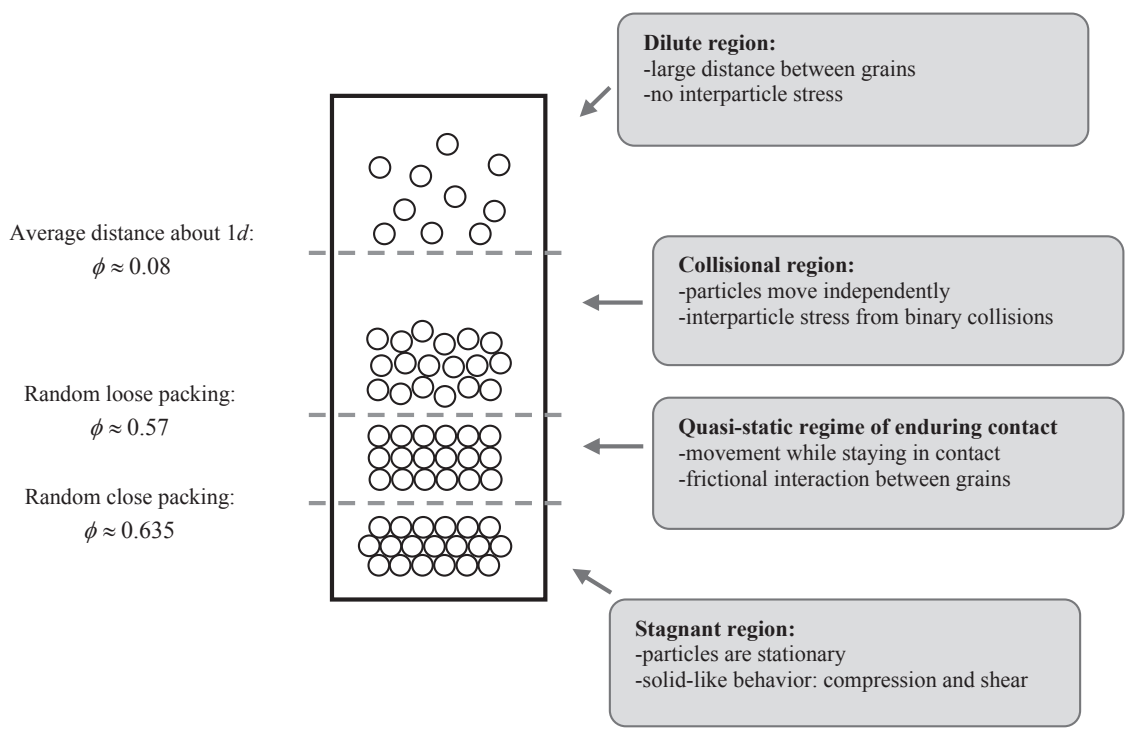

Figure 4.1: Schematization of vertical regions

In the latter equation, the first two right-hand side terms represent production of particle velocity fluctuations by shear. $Q$ represents the flux of the fluctuation energy and $\gamma$ the dissipation from inelastic collisions. The fifth term describes the effect of fluid-sediment interaction, with $2 \beta \phi \alpha k_{f}$ a source due to fluid turbulence and $-2 \beta \phi \Theta$ a sink due to drag. The symbols $p_{\mathrm{s}}, \xi_{\mathrm{s}}$ and $\mu_{\mathrm{s}}$ in the first equation denote granular pressure, bulk viscosity and shear viscosity. Note that $Q, \gamma, p_{\mathrm{s}}, \xi_{\mathrm{s}}$ and $\mu_{\mathrm{s}}$ itself are also functions of the volumetric sediment concentration $\phi$, the granular temperature $\Theta$ and properties of the sediment. See Jenkins and Hanes [1998] for further details.

In the region of enduring contact, the main assumption of the kinetic theory (binary collisions) is no longer appropriate. Therefore, additional closures are adopted in that regime (increased shear viscosity to account for the effect of frictional bonds and extra normal stress due to packed identical spheres in Hertzian contact). At the interface to the immobile bed, a Coulomb failure criterion is applied. See Hsu et al. [2004] for the complete description of the closures, parameters and boundary conditions.

\subsubsection{Solution method}

The equations are solved numerically using a grid size that is fine and uniform in the lower part of the domain (typically $\Delta z=0.3 \mathrm{~mm}$ ) and subsequently gradually increases in the upper part. The time integration makes use of both implicit and explicit discretizations. The latter put constraints on the time step $\Delta t$, which is dynamically adapted every time step to both guarantee stability and limit the 

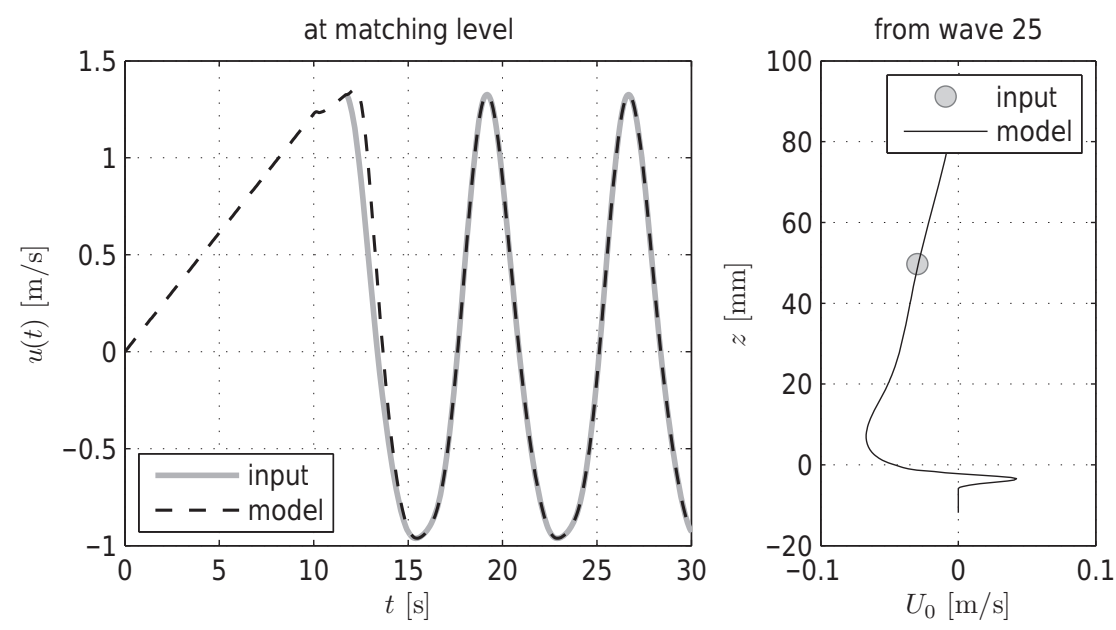

Figure 4.2: Model results for horizontal velocity $u$ compared with the velocity input. Left: time series at $z=z_{\text {match. }}$. Right: profile of mean velocity $U_{0}$ together with the mean velocity of the input signal at $z=z_{\text {match }}$.

computational time. To obtain improved stability compared to earlier model versions, the time-step criteria have been adapted to account for the $(1-u / c)$-term in front of the time derivative in case of horizontal non-uniform flow. The time integration is carried out using a predictor corrector method with all equations solved consecutively within every step. Usually 25 wave periods are simulated to ensure convergence of the (wave-averaged) results.

Another adaptation compared to earlier model versions concerns the forcing of the model. In the earlier versions, the oscillating pressure gradient in the momentum equation was computed from a prescribed oscillating velocity in the free-stream. It is now also possible to force the model to match any prescribed velocity signal $u(t)$ at $z=z_{\text {match }}$, where $z_{\text {match }}$ can be either in or outside the wave boundary layer and $u(t)$ consists of both the periodic and mean current component. This adaptation has been realized to allow for model-data comparison also for experiments with velocity profile measurement that do not extend up to the free stream. The new forcing is introduced gradually after the startup of the simulation and our experience is that a smooth and high-frequently sampled input signal is required to obtain stable results, especially for cases with high vertical orbital velocities. The quality of the match is illustrated in Figure 4.2.

\subsection{AVAILABLE DATA}

Three data sets have been selected that can be used for validation, calibration and further study of the effects of grain size variation and of flume-tunnel differences: Set 1) O'Donoghue and Wright [2004]; set 2) Dohmen-Janssen and Hanes [2002]; set 3) Schretlen [2012]. 
Set 1 contains detailed measurements of the sediment concentration $c(z, t)$ throughout the sheet-flow layer beneath oscillatory flow over various sand beds. These data have been obtained from Concentration Conductivity Measurements (CCM) in the Aberdeen Oscillatory Flow Tunnel (AOFT). Both sinusoidal and velocity-skewed oscillatory flows with varying period and energy were generated and sand beds of various compositions were investigated. This included beds of wellsorted fine, medium and coarse sized sediment (median grain size $d_{50}$ respectively $0.13,0.27$ and $0.46 \mathrm{~mm}$ ) and mixtures hereof. During the mixed-sediment experiments, an Ultrasonic Velocity Profiler (UVP) was present with which detailed information has been obtained on the horizontal velocity $u(z, t)$ inside the oscillating boundary layer.

Data set 2 and 3 are both the result of full scale wave flume experiments in the Hannover Large Wave Flume (GWK). In both cases, wave period $T$ and wave height $H$ of the regular velocity skewed waves were varied while the water depth $h$ was $3.5 \mathrm{~m}$ for all test conditions. In the experiments of Dohmen-Janssen and Hanes [2002] (set 2), the horizontal sand bed consisted of well-sorted grains with $d_{50}=0.240 \mathrm{~mm}$. Horizontal velocities were measured with an Acoustic Doppler Velocimeter (ADV) at around $100 \mathrm{~mm}$ above the still bed level and concentrations inside the sheet-flow layer were measured using CCM's. From correlation techniques, horizontal sediment velocity could be determined around maximum on- and offshore flow.

The experiments of Schretlen [2012] (set 3) contain two series, with sediment with a median grain size $d_{50}$ of $0.245 \mathrm{~mm}$ and $0.138 \mathrm{~mm}$ respectively. During both series both CCM and UVP were applied among other instruments and detailed concentration and velocity profile measurements were obtained in the sheet-flow and wave boundary layer. The UVP-measurements make these experiments the first to offer detailed information on the boundary layer flow beneath full scale waves over a mobile bed in the sheet-flow regime.

Set 1, with beds of well-sorted fine, medium and coarse sized grains, is most suitable for validation of the grain-size dependency of the model behavior for erosion depth and concentration profiles. The boundary layer velocity measurement of set 3 are unique material to validate the model's ability to reproduce boundary layer flow beneath waves, including the streaming profile. Vertical profiles of the horizontal sediment flux can in principle be obtained by combining the (UVP-)velocity and (CCM-)concentration measurements. However, the flux profiles for set 1 given by O'Donoghue and Wright [2004] are determined using the velocity information from the mixed sand tests under the assumption that these velocities are representative for the various bed conditions. Schretlen [2012] has shown that this assumption is not correct, especially for the mean current (and thus for the streaming induced flux). Quantification of the fluxes for set 3 is hampered by questions concerning the results of Schretlen [2012] for concentrations, especially for medium sized sand, after comparison with the results of Dohmen-Janssen and Hanes [2002]. For these reasons, model-data comparison on sediment fluxes will focus on predicted trends in flux profiles for fine and medium sized sand both without and with progressive wave streaming. 


\subsection{MODEL-DATA COMPARISON ON EROSION DEPTHS}

\subsubsection{Model-data comparison on grain size dependent erosion behavior}

Figure 4.3 (b) shows time-series of the erosion depth $\delta_{\mathrm{e}}$ of sand beds of well-sorted fine, medium and coarse sized sand under velocity-skewed oscillatory flow (condition FA7515, MA7515 and CA7515 of set 1, O'Donoghue and Wright [2004], with $d_{50}$ respectively $0.15,0.27$ and $0.46 \mathrm{~mm}$ ). To simulate these cases, we force the model to match the UVP-measured horizontal velocity at $49 \mathrm{~mm}$ above the original still bed level (measurements above mixed sand bed, X1A7515). The results for the erosion depth are shown in Figure 4.3 (c). Hereby, erosion depth is defined as the position of the lowest model grid point where the absolute sediment velocity is larger than zero.

The model results in Figure 4.3 (c) show erosion of the sand beds beneath both the onshore and offshore phase of the oscillatory flow. Next, there is a reduction of $\delta_{\mathrm{e}}$ during flow reversal. This is a correct reproduction of the experimentally observed sheet-flow layer behavior (panel b). However, the model results show fast and deep erosion for the coarse grains and only little erosion for the fine grains. This is not in line with the experimental results and also contradictory to what we intuitively expect for varying grain size. Nevertheless, the reduction of $\delta_{\mathrm{e}}$ during flow reversal shows a grain size dependence corresponding to the measurements: a fast drop of $\delta_{\mathrm{e}}$ for coarser sediment. So the settling characteristics reflect some of the expected grain size dependency, but the pick-up behavior of the model is not realistic.

\subsubsection{Grain-turbulence interaction (I): alternative formulations for fluctuation coefficient $\alpha$}

To improve the pick-up behavior of the model, we investigate the effect of adapted formulations for turbulence in sediment-laden flow. The argument to start any sensitivity study or review of the model formulations here, is the same as brought forward by Amoudry et al. [2008]: Sediment pickup is related to bed shear stress, unsatisfactory pick-up behavior is therefore probably related to inaccurate (time dependent) bed shear stress. The total bed shear stress is influenced both by turbulent and intergranular stresses, with increasing importance of the first for decreasing grain size. The original model [Hsu et al., 2004] has been validated on coarse grains, from which can be concluded that the intergranular stress formulations are satisfactory. Therefore, the first sub-model to be reconsidered to improve the grain-size dependent behavior is the turbulence model. Besides, the modeling of concentration effects on the carrier flow turbulence is subject of discussion in literature (e.g. [Squires and Eaton, 1994], [Amoudry et al., 2008]).

The modeled physical mechanisms of grain-turbulence interaction are buoyancy and drag. For coarse grains, the inertia of the grains is relatively large (large Stokes number) and the concentration of suspended sediment will be relatively small. In that case drag will be the most important grainturbulence interaction mechanism. Very fine particles will move easily with the flow and will result in steeper concentration profiles. In that case buoyancy will be the normative mechanism. For our model application to medium and fine sized sand $\left(d_{50}=0.27\right.$ and $\left.0.15 \mathrm{~mm}\right)$ beneath waves (wave 
period $T \approx 2-10 \mathrm{~s}$, near-bed free stream velocity amplitude $\hat{u}_{\infty} \approx 1 \mathrm{~m} / \mathrm{s}$ ), we not only enter the parameter range where turbulent stresses are increasingly important over intergranular stresses for sediment pick-up, but also the range where both drag and buoyancy are relevant (see appendix $\mathrm{G}$ for a discussion on the Stokes number range).

Model parameters related to buoyance and drag terms in the $k-\varepsilon$ turbulence model (respectively the fourth and fifth RHS-term in equation (4.10) and (4.11)) are $C_{\varepsilon 3}, \sigma_{\mathrm{c}}, \alpha, \beta$ and $n$. Here, we focus on fluctuation coefficient $\alpha$ in the drag terms. The reason therefore is threefold. Firstly, preliminary
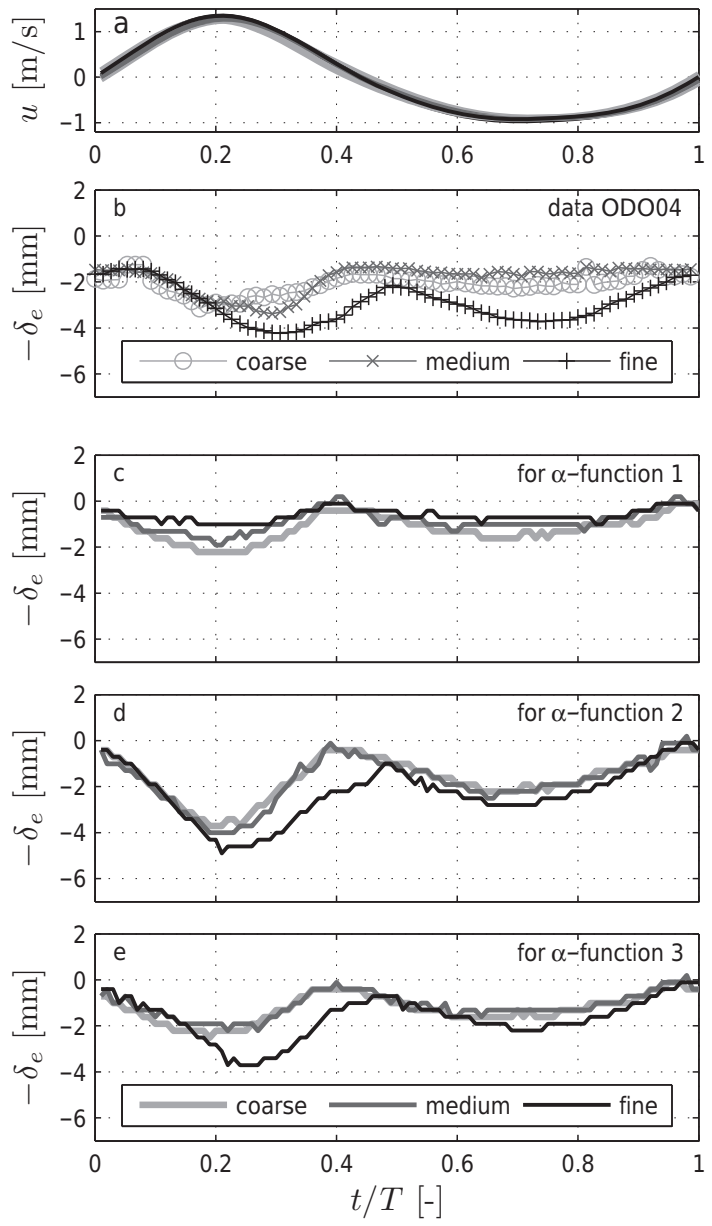

Figure 4.3: Erosion depth $\delta_{\mathrm{e}}$ for sand beds of coarse $\left(d_{50}=0.46 \mathrm{~mm}\right)$, medium $(0.27 \mathrm{~mm})$ and fine $(0.15 \mathrm{~mm})$ sized sand for condition A7515 of O'Donoghue and Wright [2004]. Panel b) experimental results. Panel c) model results. Panel $\mathrm{d} \&$ e) model results with alternative formulations for fluctuation coefficient $\alpha$. $\alpha$ function 1: equation (4.12); $\alpha$-function 2: equation (4.16) (here with $B=0.15$ ); $\alpha$-function 3: equation (4.17), (here with $c_{1}=0.5$ and $c_{2}=1.5$ ). Panel a) horizontal fluid velocity at $z=z_{\text {match }}$; 
sensitivity tests showed a large sensitivity of the model results to the drag terms: fine sand beds were eroded tens of mm with the drag terms multiplied by 0.5 . Secondly, alternative expressions for $\alpha$ are given in literature. Thirdly, the present $\alpha$-function can be questioned based on theoretical considerations.

Danon et al. [1977] and Chen and Wood [1985] proposed and exponential function for the fluidparticle fluctuation correspondence ( $\alpha$-function 2 ):

$$
\alpha=\exp \left(-B T_{p} / T_{t}\right)
$$

with $B$ an empirical coefficient of about 0.08 . Next to that, we introduce $\alpha$-function 3:

$$
\alpha=\left[1+c_{1}\left(T_{p} / T_{t}\right)^{c_{2}}\right]^{-1}
$$

with coefficients $c_{1}$ and $c_{2}$ available for tuning, provided that $c_{2}>1.0$. The consideration behind this function is as follows: Small particles will follow the fluid motion completely, while large particles are hardly accelerated by a velocity difference. Therefore any $\alpha$-function should be 1 for infinitesimal small $T_{p} / T_{t}$ and approach zero for infinitively large $T_{p} / T_{t}$. However, it may also be expected that no effect of drag on turbulence is present for particles perfectly following the fluid fluctuation. This is not the case when applying $\alpha$-function 1 or 2 from equation (4.12) and (4.16). This becomes clear when we substitute equation (4.13), $\beta=\rho_{\mathrm{s}} / T_{p}$, into the drag terms of the turbulence model: the damping effect of drag on $k$ and $\varepsilon$ turns out to be proportional to $(1-\alpha) / T_{p}$. Using $\alpha$ from equation (4.12) and (4.16), (1- $\alpha) / T_{p}$ does not approach zero for infinitesimal small $T_{p} / T_{t}$, but respectively $1 / T_{t}$ and $B / T_{t}$. Figure 4.4 shows $\alpha$ and (1- $\left.\alpha\right) / T_{p}$ as function of $T_{p} / T_{t}$ for the three alternative $\alpha$-functions.

\subsubsection{Results for erosion depths with alternative $\alpha$-functions}

Figure 4.3 panel (d) and (e) show model results obtained with $\alpha$-function 2 and 3 (values of coefficients $B, c_{1}$ and $c_{2}$ have been tuned). In line with the data, (d) and (e) show the largest erosion depths for fine sand beds. This is an important improvement over the original results (c). For coarse and medium grains, the minimum erosion depth coincides with the reversal of the flow (see panel a). For fine sand, this minimum occurs later. Furthermore, the minimum erosion is larger. Also these features of the model results are consistent with the data. They reflect the fine sand phase-lag behavior: the slow settling of fine sediment causes large amounts of sand still in suspension at the moment of flow reversal. The effect of larger fall velocity of medium and coarse grains is most clearly visible in panel (d), where the erosion depths reduce the strongest after maximum onshore flow. Some model-data differences are the complete return of the immobile bed to the initial still bed level $\mathrm{z}=0$ during flow reversal in the medium and coarse sand simulations, and the larger difference between the fine sand erosion maxima beneath the two velocity peaks in the model results compared to the data. 

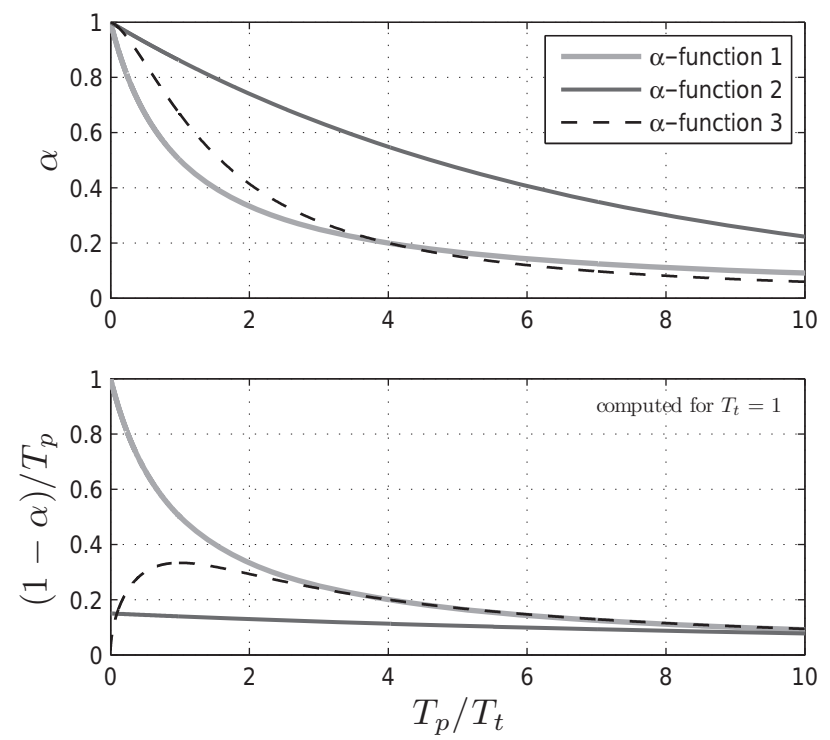

Figure 4.4: Particle-fluid fluctuation coefficient $\alpha$ according to equation (4.12), (4.16) and (4.17) and the accompanying damping proportionality $(1-\alpha) / T_{p}$ as function of the relative particle response time $T_{p} / T_{t}$. Settings: $B=0.15 ; c_{1}=0.5 ; c_{2}=1.5$. Computations with $T_{t}=1.0$.

We conclude that the alternative $\alpha$-functions induce an improved grain size dependent erosion behavior of the model. We explain this improvement from a reduction of drag induced turbulence damping, especially for simulations with fine grains: For identical $T_{t}$, a decreasing grain size results in a decreasing $T_{p} / T_{t}$ (see equation (4.13) and (4.6)). For smaller $T_{p} / T_{t}$, the alternative $\alpha$-functions show less damping effect, i.e. smaller $(1-\alpha) / T_{p}$, than the original one (see Figure 4.4). Note that estimation of the effect of changes in the turbulence model is complicated by the fact that $T_{t}$ actually varies strongly with position and time and is itself also influenced by the drag effect. In the remainder of this study, $\alpha$-function 2 will be used as default. $B$ is used as tuning coefficient and set to 0.18 .

Further improvement may be achieved by investigating more alternative $\alpha$-functions. Note that it may also be possible to derive such a function from a transfer function describing the relation between particle and fluid fluctuations. The present $\alpha$-function used to model the grain - carrier flow turbulence interaction is actually only a real amplitude ratio (see appendix F). A complex transfer function would give a better account for the phase difference between fluid and particle motions in high-frequency oscillations. For this, transfer functions as in appendix $\mathrm{G}$ could be applied (see also Hinze [1975], Hjelmfelt and Mockros [1966]). This is not further elaborated here. 


\subsection{MODEL-DATA COMPARISON ON CONCENTRATION AND VELOCITY}

The next step in the model-data comparison comprises a validation on time-dependent concentration profiles and on both time-dependent and wave-averaged velocity profiles. In line with section 3, the first will be carried out with the concentration profiles measured by O'Donoghue and Wright [2004] for both fine and medium sized sand. The velocity-validation will focus on the velocity profiles measured by Schretlen [2012]. Hereby, we especially look to the wave-averaged velocity, to check the model's ability to reproduce progressive wave streaming.

\subsubsection{Time-dependent concentration profiles for medium and fine sized sand}

Figure 4.5 and Figure 4.6 show a comparison of measured and computed time-dependent sediment concentration profiles at various phases of a velocity skewed oscillatory flow for respectively medium and fine sized sand (condition MA7515 and FA7515 of O'Donoghue and Wright [2004]). In the figures, phase $t / T=0.0$ marks the beginning of onshore flow in the free stream, compare Figure 4.3 panel (a).

From Figure 4.5 and Figure 4.6 we observe that the vertical position of the toe of the concentration profile, where $\phi=0.6$, is followed reasonably well by the model, especially for medium sized sand. This result is in line with Figure 4.3 for the erosion depth $\delta_{\mathrm{e}}$. Next, the model results show a decreasing $\phi$ beneath the original still bed level and an increasing $\phi$ above during increasing (absolute) flow velocities, which is also consistent with the data. For the overall profile, the best model-data agreement is found during offshore flow, in particular for medium sized sand. The simulated profiles generally show a large vertical concentration gradient just above the instantaneous erosion depth and a smaller concentration gradient at higher levels. The difference/transition between the two parts of the profile is the strongest for fine sand, where almost horizontal profiles are observed at low elevations. This is a discrepancy with the data, which show a more constant gradient over the sheet-flow layer. It seems that especially the fine sediment is brought to high levels in the water column directly after mobilization, leaving behind the pick-up layer with low concentrations. Another remarkable issue is observed during onshore to offshore flow reversal $(t / T=$ 0.42 ) in the fine sand simulation. Following the profile from the bed upwards we see subsequently a small concentration decrease around $-2 \mathrm{~mm}$, a thin unstably stratified layer, followed by a very strong concentration decrease: a nearly horizontal profile. The strong gradient close to $z=0$ implies that the concentration profile has collapsed nearly completely. The unstable stratifications are probably a secondary, numerical effect of the strong gradient.

Similar to the erosion depth, also concentration profiles are highly sensitive to the turbulence stresses. Therefore, the remaining profile imperfections might be caused by remaining inadequacies in the turbulence model. Without turbulence measurements available, we try to check the turbulence model using the (ensemble-averaged) velocity profiles. 

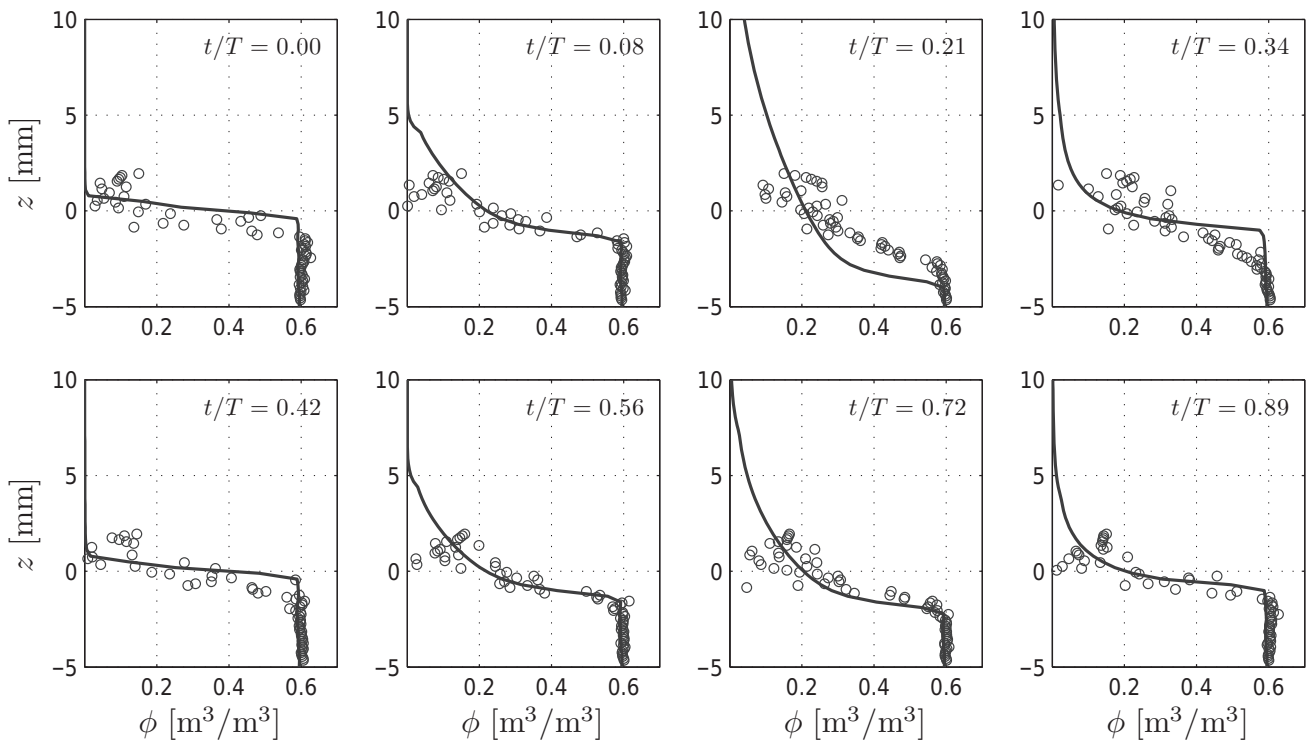

Figure 4.5: Comparison of measured (blue circles) and computed (black line) sediment concentrations at various phases of the flow for case MA7515.
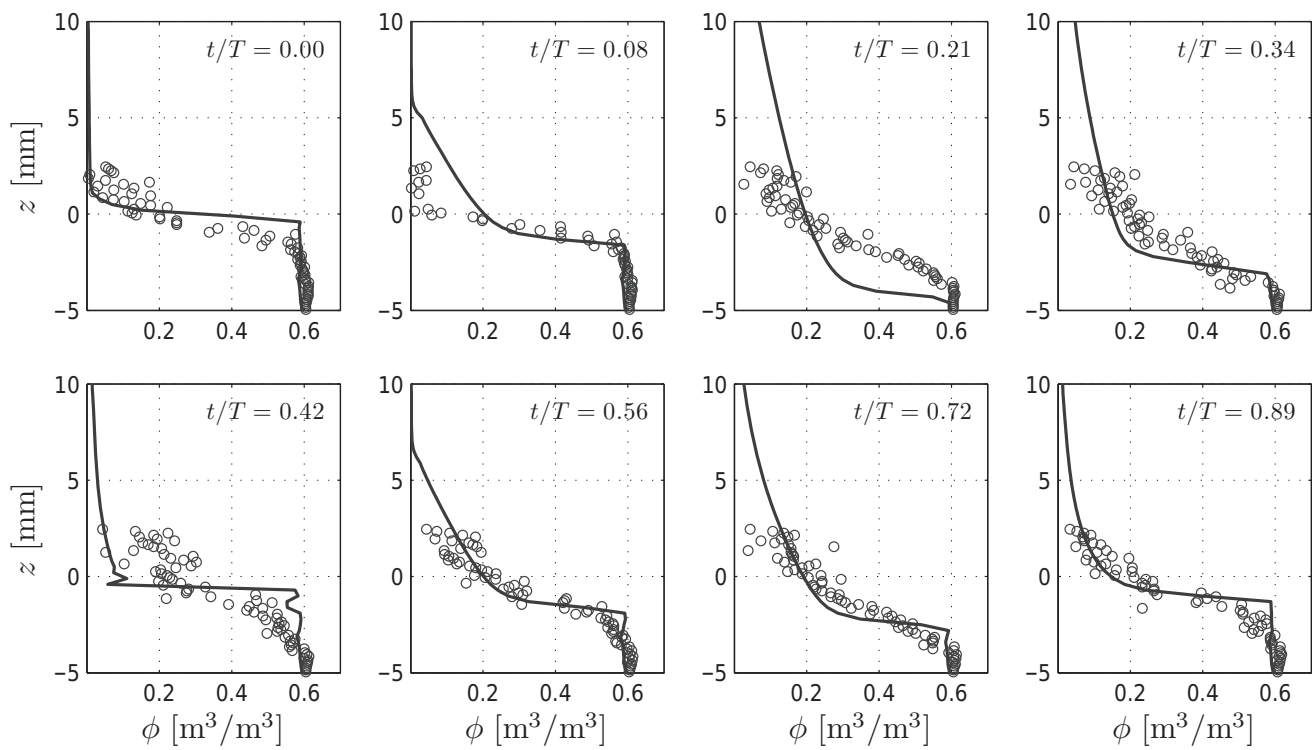

Figure 4.6: Comparison of measured (blue circles) and computed (black line) sediment concentrations at various phases of the flow for case FA7515 


\subsubsection{Time-dependent and wave-averaged velocity profiles}

Figure 4.7 shows a model-data comparison for vertical profiles of horizontal velocity from the bed to the free stream at various phases of the flow. Note that the velocity measurements of O'Donoghue and Wright [2004] shown here have been obtained from the mixed sediment experiments, while the simulation results belong to fine sand case FA7515.

Firstly, we observe that the course of the velocity profile from the bed to the free stream is generally followed well by the model. This includes the phase lead of the near-bed velocity over the free stream (most clear for $t / T=0.0$ and 0.42 ), and the location in the upper part of the profile above which velocity shear is nearly absent. The latter means that in general the model is well capable to predict the boundary layer thickness, and thus the turbulence intensity. Model-data differences are the largest for $t / T=0.0$ and 0.08 , i.e. around the off- to onshore flow reversals and during the following acceleration from zero towards maximum onshore flow. Here, the simulated profiles show a kink and the velocity gradients in the lowest part of the domain are overestimated. These features point at an underestimation of the vertical momentum transfer in this area, which might be explained by underestimated turbulence intensities near the bed.

Figure 4.8 shows a comparison of measured and computed horizontal velocities at various phases of the wave both for condition Re1575fine (run 173) from the fine sand series of the flume experiments of Schretlen [2012]. Comparing model results and data, we observe that also in this case the phase lead and location of no shear are reproduced by the model. The overestimation of the near bed velocity gradients in the acceleration phase observed in Figure 4.7 is almost absent here. However, the kink in the velocity profile is present again. In this case, the latter is still present both during maximum onshore and maximum offshore flow velocity $(t / T=0.17$ and 0.68 respectively). The longer persistence might be related to the stronger acceleration in this slightly more energetic flow condition. Finally, except for a slight underestimation during the acceleration phase $(t / T=0.09$ and 0.56), the erosion depths are reproduced well.

Figure 4.9 shows a model-data comparison for streaming profiles from both tunnel and flume conditions with beds of both fine and medium sized sand. Most important observation is that the model clearly produces the onshore mean current beneath the original still bed level, resulting from differences in erosion depth between on- and offshore flow. The reproduction of this typical sheetflow layer characteristic is an important improvement compared with earlier streaming profile predictions [Kranenburg et al., 2013] (chapter 3). Next, both model and data show a strong velocity gradient and an offshore current just above the original bed level. This current (wave shape streaming) is explained from differences in turbulence intensity between on- and offshore flow beneath the velocity-skewed waves [Trowbridge and Madsen, 1984], [Kranenburg et al., 2012]. Reproduction of this feature indicates good model validity concerning the turbulence asymmetry. This is noteworthy, considering the underestimation of turbulence viscosity suggested by Figure 4.7 and Figure 4.8. Next, note the local minimum around $z=20 \mathrm{~mm}$ in both model results and data in panel $b$. This feature is explained from an onshore directed streaming contribution in the presence of 

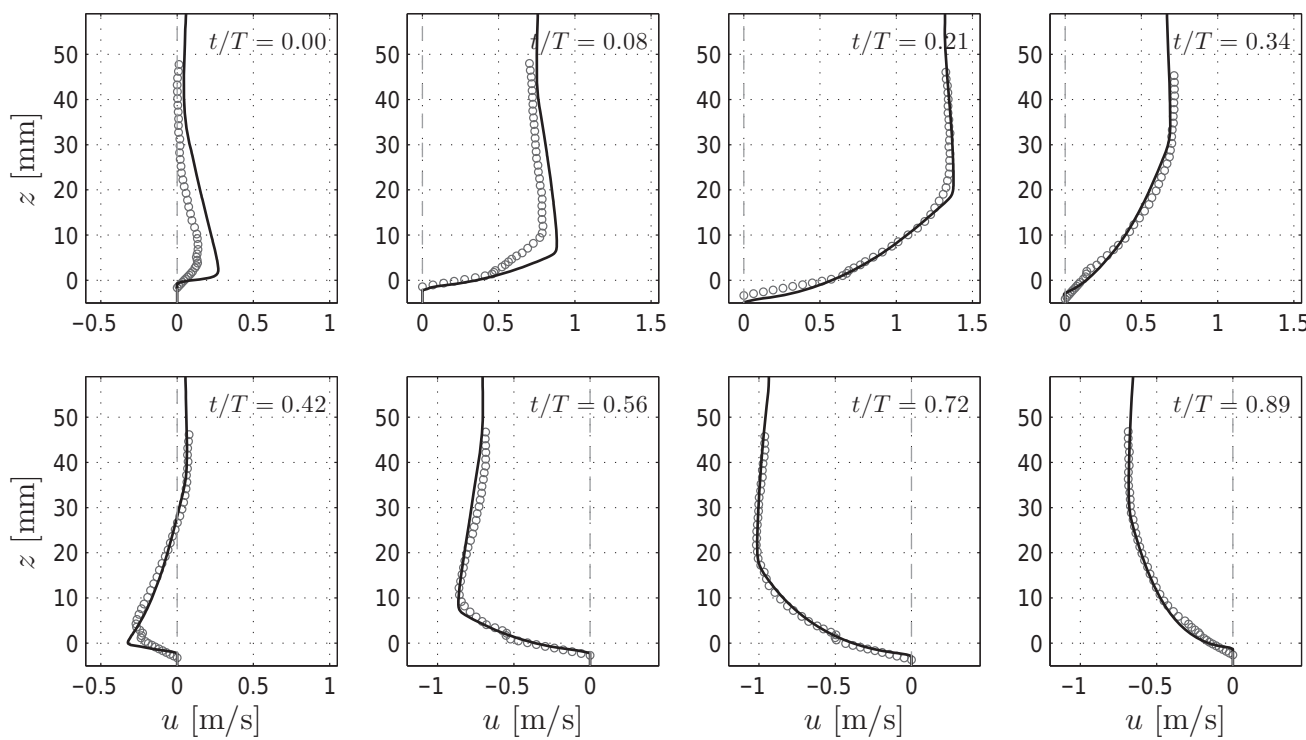

Figure 4.7: Comparison of measured (blue circles) and computed (black line) horizontal sediment velocities at various phases of the flow. Measurements: X1A7515; Model simulations: FA7515. $Z_{\text {match }}=40 \mathrm{~mm}$.
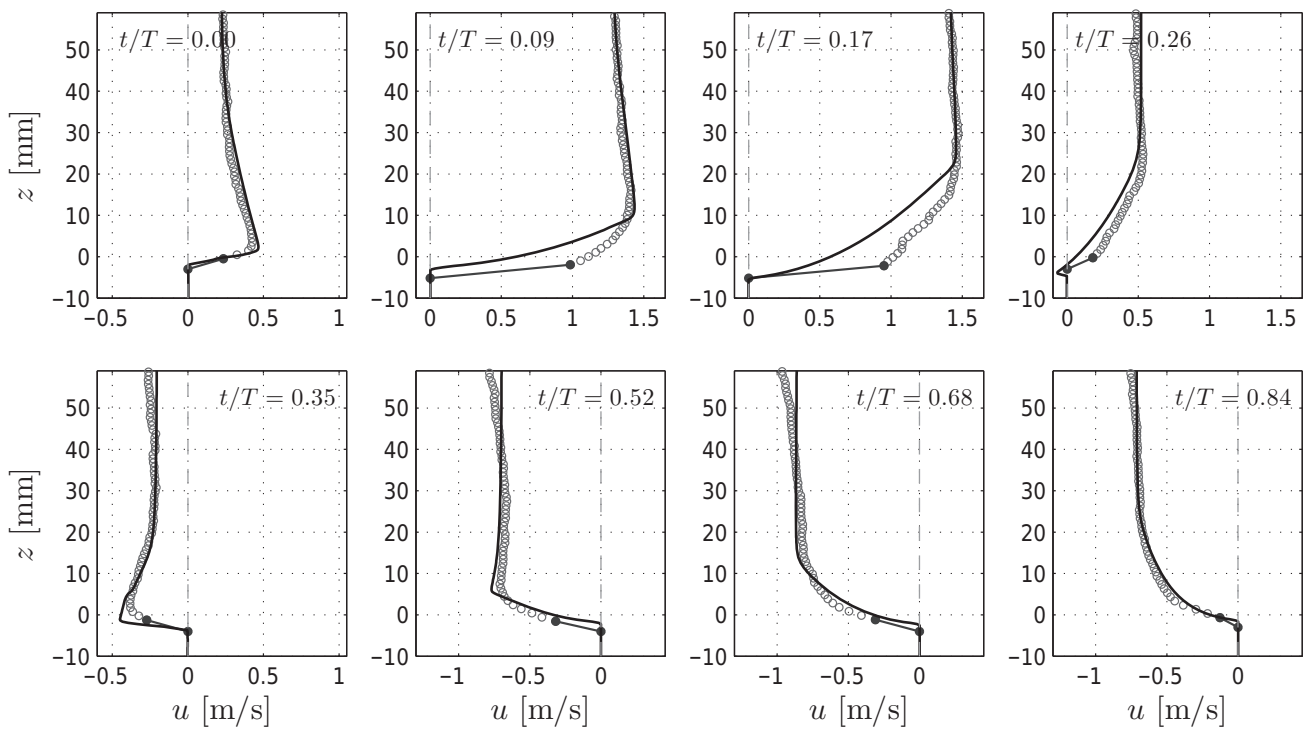

Figure 4.8: Comparison of measured (blue circles) and computed (black line) horizontal velocities at various wave phases for surface wave condition Re1575fine (run 173). $Z_{\text {match }}=40 \mathrm{~mm}$. 
(a) A7515

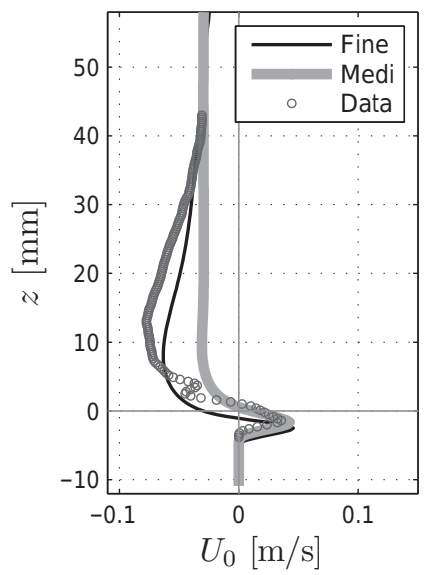

(b) Re1575fine

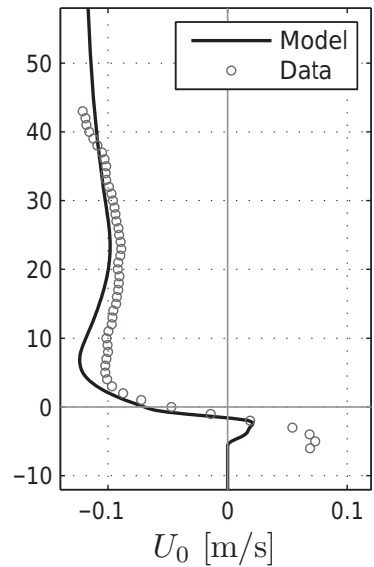

(c) Re1575medi

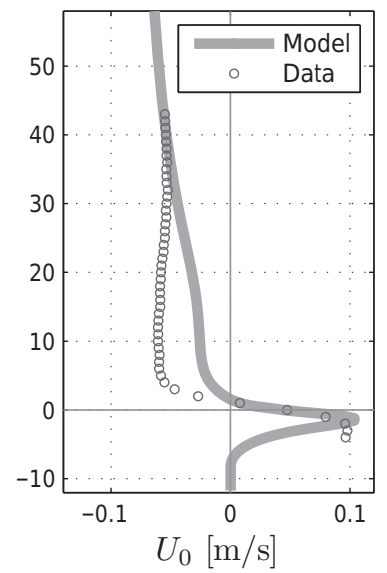

Figure 4.9: Comparison of measured and computed wave-averaged horizontal velocity $U_{0}$. Panel a) Simulation for tunnel conditions FA7515 (fine) and MA7515 (medium sized sand) compared with the measured profile from condition X1A7515 (sand mixture). Panel b) and c) Flume conditions with fine and medium sand. $Z_{\text {match }}=40 \mathrm{~mm}$ in all simulations.

vertical orbital motions (progressive wave streaming), which, considering the result, is also reproduced correctly.

\subsubsection{Evaluation}

The model reproduces a number of important experimentally observed sheet-flow and boundary layer characteristics. This includes the boundary layer thickness, the phase lead of the near bed flow, the wave shape streaming and the progressive wave streaming. It also includes the erosion depth asymmetry and the connected onshore current in the bottom part of the sheet-flow layer (the pick-up layer). Next, also the tilting behavior of the concentration profile (decreasing concentrations beneath and increasing concentrations above the original still bed level during increasing (absolute) flow velocities) is reproduced. With this feature, the model shows typical sheet-flow layer behavior. Remaining inaccuracies in the model results concern in particular the shape of the predicted concentration profile during maximum flow, the collapse of the profile for fine sand during flow reversal, and the kink in the velocity profile during flow reversal and subsequent acceleration. Although occurring at flow reversal, the collapse of the concentration profile is potentially important to fluxes of fine sand. This is because it suppresses phase lag effects, i.e. offshore transport of sand mobilized during onshore flow, the mechanism that explains the offshore transport rates found in tunnel experiments. Both the velocity and concentration profile inaccuracies around flow reversal might be explained by underestimated near bed turbulence intensities. Therefore, improved predictive model skills might be obtained from further adaptations to the turbulence model. 

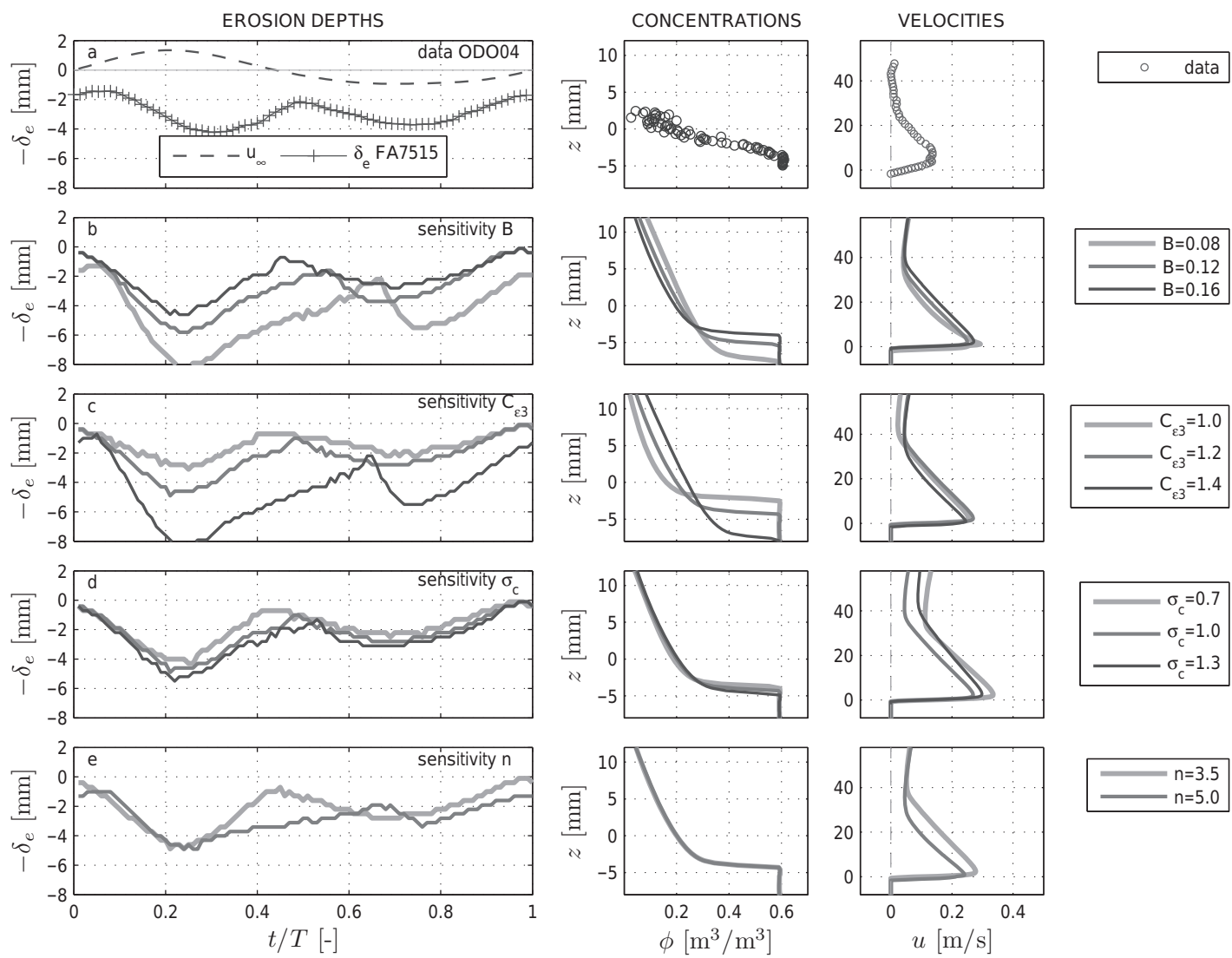

Figure 4.10: Sensitivity tests for coefficients in the grain - carrier flow turbulence interaction terms. Column 1) Time varying erosion depth $\delta_{\mathrm{e}}$; Column 2) concentration profile at $t / T=0.21$, maximum onshore flow; Column 3) velocity profile at $t / T=0.02$, just after offshore to onshore flow reversal. Row a) data condition FA7515 of O'Donoghue and Wright [2004]; Row b,c,d,e respectively sensitivity tests for coefficients $B, C_{\varepsilon 3}$, $\sigma_{\mathrm{c}}$ and $n$.

\subsubsection{Grain-turbulence interaction (II): further sensitivity tests}

This section further discusses the sensitivity of the model results for coefficients / parameters in the turbulence model. Hereby, we focus again on the model terms related to the grain - carrier flow turbulence interaction. The model parameters related to buoyance and drag terms in the $k-\varepsilon$ turbulence model (respectively the fourth and fifth RHS-term in equation (4.10) and (4.11)) are $C_{\varepsilon 3}$, $\sigma_{\mathrm{c}}, \alpha, \beta$ and sub-coefficients $n$ and $B$ (from $\alpha$-function 2).

Figure 4.10 shows the results for modification of $B, C_{\varepsilon 3}, \sigma_{\mathfrak{c}}$, and $n$. The figure presents for each test a time series of the erosion depth $\delta_{\mathrm{e}}$, a vertical profile of sediment concentration $\phi$ during maximum onshore flow and a vertical profile of fluid velocity $u$ just after off- to onshore flow reversal. All 
tests simulate condition FA7515 of O'Donoghue and Wright [2004]. The results for the erosion depth show that the erosion during maximum on- and offshore flow increases both with decreasing $B$ and with increasing $C_{\varepsilon 3}$ or $\sigma_{\mathrm{c}}$. We explain this erosion behavior as follows: An increasing $\sigma_{\mathrm{c}}$ leads to a decreasing reduction of $k$ from the buoyancy term, equation (4.10), term 4 . A decreasing $B$ leads to a decreasing reduction of $k$ from drag. (Also the reduction of $\varepsilon$ from drag will decrease, which actually increases the dissipation rate of $k$, but the effect hereof on $k$ during maximum flow appears small compared to the direct effect). An increasing $C_{\varepsilon 3}$ leads to increasing reduction of $\varepsilon$ and thus an decreasing dissipation rate of $k$. For all these changes, the larger turbulent kinetic energy induces increased bed shear stresses, leading to larger erosion depths. An increasing $n$ affects the model results in various ways through the drag coefficient $\beta$. The dominant effect is the reduction of the settling velocities. This causes a slower return of the bed level towards the initial still bed level. Note that this will result in increased phase lag effects.

The main conclusions from the sensitivity tests is that the maximum erosion depth $\delta_{\mathrm{e}}$ is relatively sensitive for changes in $B$ and $C_{\varepsilon 3}$ and that the return speed of the bed level to the initial still bed level is largely affected by $n$. However, the shape of the concentration profile during maximum onshore flow and of the velocity profile just after flow reversal is not really affected by changes in these parameters. Based on the latter observation, we recommend further research on the behavior of the model around flow reversal. We return to this issue in the discussion. Next, also alternative expressions for drag parameter $\beta$ are available in literature which could be included in this sensitivity analysis.

\subsection{SEDIMENT FLUXES FOR FINE AND MEDIUM SIZED SAND IN TUNNEL AND FLUME}

Next, we apply the model to investigate trends in sediment flux profiles under influence of grain size variation and free surface effects. Hereto, we compare the sediment flux profiles computed for condition MA7515 and FA7515 of the oscillating flow experiments of O'Donoghue and Wright [2004] with simulations for these same conditions, but now including the effects of the horizontally non-uniform flow field under progressive waves (which is realized by including again the advective terms in the fluid and sediment momentum and fluctuation energy equations).

Figure 4.11 shows profiles of the instantaneous sediment flux both during maximum onshore flow (a) and maximum offshore flow (b), together with profiles of wave-averaged sediment fluxes (c). The first row shows results for medium sized sand, the second row for fine sized sand. Each panel contains flux results obtained from an oscillating flow simulation, a progressive wave simulation and the flow tunnel experiments, where the latter are obtained by multiplication of the condition specific concentration measurements with the velocity measurements for condition X1A7515.

Firstly, note that the oscillatory flow simulations produce instantaneous and period-averaged sediment flux profiles with an order of magnitude and profile shape comparable to the results of the tunnel experiments. This is the case both for the medium and fine sized sand cases. The period 
(a)

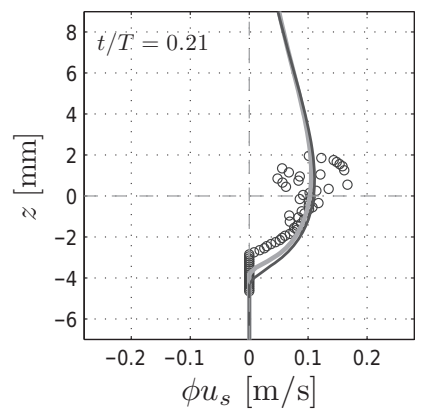

(a)

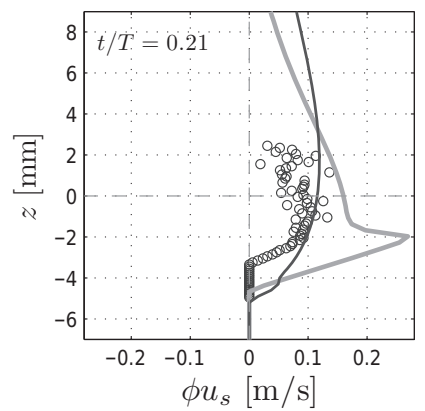

(b)

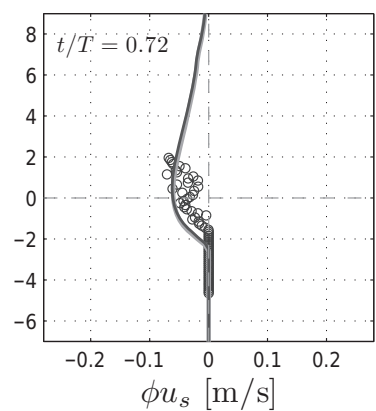

(b)

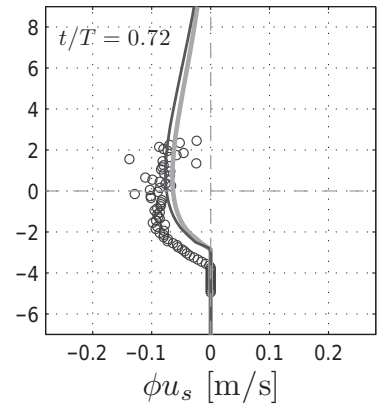

(c)

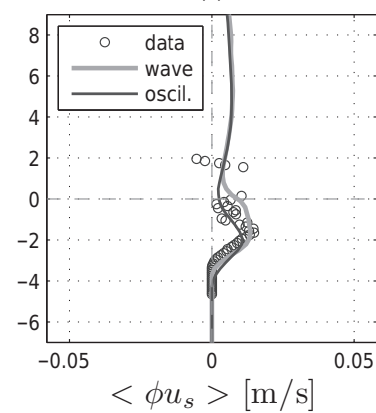

(c)

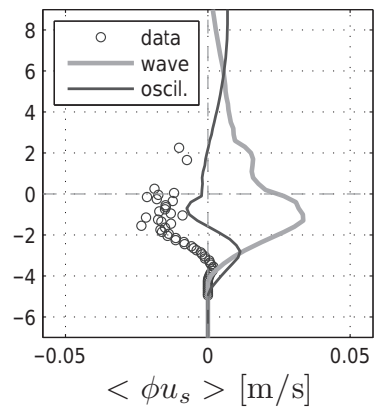

Figure 4.11: Vertical profiles of the horizontal sediment flux $\phi u_{s}$. (a) during maximum onshore flow; (b) during maximum offshore flow; (c) period averaged. Row 1) for medium sized sand condition MA7515; Row 2) for fine sized sand condition FA7515. Blue circles: oscillatory flow tunnel experiment; black line: oscillatory flow simulation; gray line: progressive wave simulation.
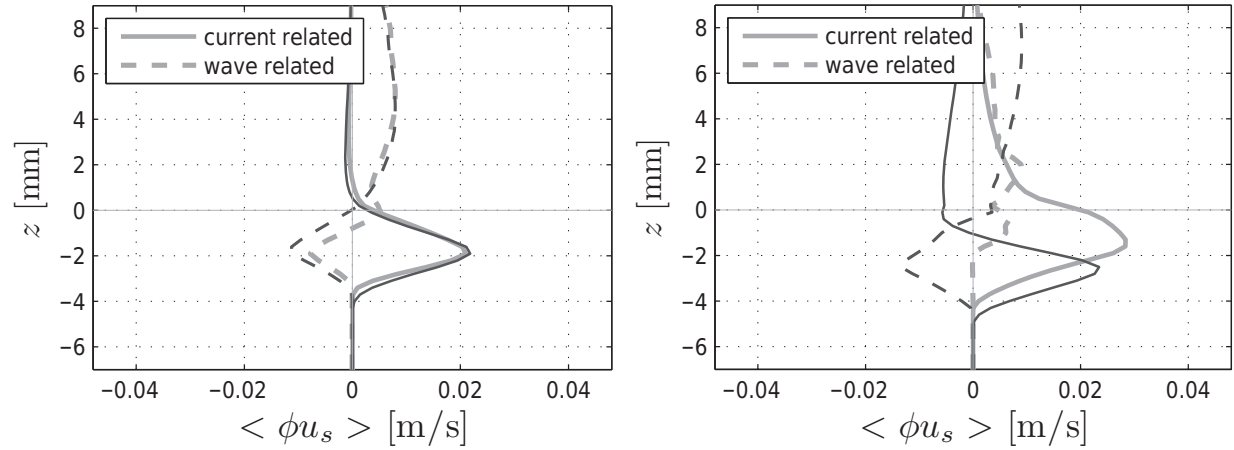

Figure 4.12: Current related contribution (continuous line) and wave-related contribution (dashed line) to the period averaged sediment flux $\left\langle\phi u_{s}\right\rangle$. Left) for medium sized sand condition MA7515; Right) for fine sized sand condition FA7515. Black lines: oscillatory flow simulations; Gray lines: progressive wave simulations. 
averaged results for fine sand are slightly shifted in onshore direction compared to the data. Note that this is directly connected to the earlier observation that for the fine sand condition the erosion depth asymmetry in the model results is larger than in the data. Next, comparison between the results for oscillatory flow and progressive waves over medium sized sand beds (row 1) shows that the differences in the instantaneous profiles are only small. The period-averaged differences are relatively larger, showing an increased onshore sediment transport rate under progressive waves. For fine sand, we find increased onshore sediment fluxes from progressive waves during maximum onshore flow (2a), especially in the lower part of the profile. Also the period averaged sediment flux is much larger and clearly onshore directed (2c). Finally, this oscillatory flow - progressive wave difference for fine sand is very large compared to the difference for medium sized sand.

These observations confirm the trend observed by Schretlen [2012] for increased onshore transport rates in flume experiments compared to tunnel experiments. Note that the trend for increased difference between the erosion depth under maximum onshore and maximum offshore flow in flumes compared to tunnels, also observed by Schretlen [2012] for velocity-skewed waves / oscillations, is not reproduced by the model results.

In Figure 4.12, the period-averaged sediment flux $\left\langle\phi u_{s}\right\rangle$ is divided in a current related and waverelated component, respectively $\langle\phi\rangle\left\langle u_{s}\right\rangle$ and $\left\langle\tilde{\phi}_{u_{s}}\right\rangle$ to study the background of the found differences. Clearly, the most important difference appears in the current-related contribution to the fine sand sediment flux (right panel). However, for fine sand, also the wave-related contribution is affected. Note that below the initial still bed level in general the current-related flux is onshore directed, while the wave-related flux is offshore directed. Schretlen [2012] explains the first as a result of the erosion depth asymmetry under velocity skewed waves / oscillations. The second observation can be explained from a negative correlation between wave-related velocities and concentrations inside the pick-up layer: high onshore velocities coincide with sediment pick-up, and thus sediment concentrations inside the pick-up layer lower than averaged.

\subsection{DISCUSSION}

The results of this study, especially section 6, provide valuable insights in the behavior of the sheetflow layer due to grain size variation and free surface effects. For sediment transport prediction within morphodynamic modeling systems, it will be very useful to further quantify and parameterize the wave-induced erosion depths and sediment fluxes, and the flux distribution over the vertical profile. Our exploration shows that this two-phase model can become a valuable instrument to do this. At present, the main hindrance for predictive model employment over a range of grain sizes are the inaccuracies in the concentration and velocity profiles predictions around and directly following flow reversal, especially for the finer grains. What possibilities are present for further model improvement and what potential limitations do exist for application in our domain of interest? 
Concerning the model formulations, it should be noted that the particle stress closure model has been originally formulated for heavy grains in the collisional regime [Jenkins and Hanes, 1998]. It is a question whether this closure from collisional theory is still valid in our domain of interest. On the other hand, the importance of this part of the model formulations decreases with decreasing grain size and it was recently shown by Amoudry [2012] for medium sized grains and moderate flow that these stress closures could be exchanged with alternative formulations without noteworthy effect on the results. Another option for improvement might be to reconsider the model formulations on the level of the momentum balances: presently, added mass forces and lift forces are not considered, while they are included in other two-phase models [e.g. Li et al., 2008]. These terms could be implemented in the present model. We expect the most from implementing the lift force: the transfer functions of appendix G suggest that on the phase-ensembled time scale, added mass effect is of minor importance.

The large sensitivity of the model results for the turbulence closure advocates further evaluation of this part of the model formulations. Firstly, more alternatives for the $\alpha$-function could be investigated, e.g. correlation functions directly derived from the transfer functions, see e.g. Hinze [1975] and appendix G. Also alternative formulations are available for the drag parameter $\beta$. Next, it needs reconsideration whether we can uphold the clear fluid turbulence coefficients for the noninteraction terms. Simultaneously, there are more fundamental question concerning the validity of any $k-\varepsilon$-model in the high-concentration region, where the flow might become laminar, and around flow reversal in rather high frequent oscillations, where a temporary strong reduction of $k-\varepsilon$ turbulence production term occurs. With our application, we might be pushing the $k$ - $\varepsilon$-model beyond its limits: features of shear instabilities [Carstensen et al., 2010] [Henriquez et al., 2012] and short and sudden concentration peaks around flow reversal [O'Donoghue and Wright, 2004] have been observed in wave boundary layer experiments. These feature can in principle not be captured by the $k$ - $\varepsilon$-turbulence model, and their importance may increase with decreasing grain size (see also Ozdemir et al. [2010], who predicted the turbulence generation during flow reversal using a turbulence-resolving model, and Guizien [2003], who modeled the concentration peak with an adaptation to the original k- $\omega$ model of Wilcox [1994]). Further research is needed to determine whether improved predictions can be achieved within the concept of turbulence-averaged continuous two-phase models.

\subsection{CONCLUSION}

In this study, we explored the possibilities to predict erosion depths and sediment fluxes, and their dependency on grain size and streaming with a two-phase continuum model. During this study, we improved two aspects of the model formulations: (1) the model forcing; (2) the way the model accounts for grain effects on fluid turbulence. The first adaptation makes it possible to force the model to match a measured velocity time-series, either in or outside the wave boundary layer. With the second adaptation, we extend the validity of the model towards finer grain sizes: the grain size dependent behavior of the model is largely improved and good reproductions are obtained of measured erosion depths of fine, medium and coarse sized sand beds. 
We conclude from model-data comparison on concentration and velocity profiles that the model is able to reproduce a number of important sheet-flow and boundary layer characteristics. An important characteristic is the tilting motion of the concentration profile during the wave. This is important, because this behavior reflects the typical structure of the sheet-flow layer, with respectively a pickup layer with decreasing concentrations and an upper sheet-flow layer with increasing concentrations under increasing (absolute) flow velocities. As a result, the model is able to produce fluxes over the entire sheet-flow layer, also below the original bed level. Next, the model reproduces the phase lead of the near-bed flow over the free stream and the maximum thickness of the boundary layer. We also find the experimentally observed positive streaming inside the sheet-flow layer and the negative current at slightly higher levels, both resulting from velocity-skewness. The model reproduction of the first feature is an important improvement over earlier streaming profile predictions.

Remaining inaccuracies mainly concern the behavior of concentration and velocity during flow reversal in fine sand simulations. From a sensitivity analysis to identify possibilities for model improvements, we conclude that the model results, especially the erosion depth $\delta_{\mathrm{e}}$, are very sensitive to changes in the grain - carrier flow turbulence interaction and the modeling of hindered settling effects.

In a model investigation on trends in sediment flux profiles under influence of grain size variation and progressive wave effects, we found period-averaged sediment fluxes increasingly onshore directed under influence of wave progression, both for medium and fine sand. This is consistent with the trends observed experimentally by Schretlen [2012]. We conclude from decomposition of the period-averaged sediment flux profile in a current-related and wave-related contribution, that the major part of the increased onshore transport of fine sand can be attributed to the current-related contribution.

We conclude from this study that the present two-phase model can become a valuable instrument for further study and parameterization of sheet-flow layer processes. We recommend further effort to improve the predictive model skills, to cover the entire range of realistic sand grain sizes on the foreshore. We especially recommend further research on the turbulence model and its behavior around flow reversal. 


\section{DISCUSSION}

This thesis investigates the effects of progressive waves on flow velocities and sheet-flow sand transport processes by numerical modeling of the wave boundary layer. In this chapter we reflect on the methodology and discuss the implications of the present study. Firstly, we discuss assumptions behind the applied modeling concepts and otherwise neglected aspects (section5.1). Secondly, we discuss methods to include our results in morphodynamic modeling systems (section 5.2). Next, we illustrate the potential implications of our results on predictions of cross-shore morphology (section 5.3) with a simple morphodynamic computation. Finally, we discuss the value of insights developed during this study for another application in the field of seabed morphology (sand waves, section 5.4).

\subsection{ASSUMPTIONS AND LIMITATIONS}

In the formulation of the numerical models applied in this study, a number of assumptions have been adopted. This concerns e.g. assumptions on the bed, the waves, the type of flow inside the wave boundary layer or the grain size distribution. Notwithstanding the reasons for the various assumptions, it is important to realize which processes are hereby excluded and how these processes would affect our results. Next, there are also processes that in principle can be dealt with by our numerical modeling tools, e.g. acceleration skewness and wave irregularity, but that were neglected in this study for other reasons. What would be the effect of these processes and how would they interact with progressive wave streaming and the other free surface effects? For a number of assumptions and neglected aspects, these questions are discussed below.

\subsubsection{Flat bed assumption}

This study focusses on progressive wave effects on sheet-flow sediment transport. It is a characteristic of sheet-flow that bed forms are washed away [Ribberink and Al-Salem, 1994]. Therefore, the flat bad assumption in our modeling tools is well justified. However, in the adjacent rippled-bed regime, the water and sediment motion change drastically under influence of the bed forms. This is induced by vortices generated on the ripple flanks around the moment of flow reversal. Firstly, these vortices cause a reduction of the contribution from progressive wave streaming to the mean current because of changes in the phase relationship between the horizontal and vertical component of the orbital velocity [Davies and Villaret, 1999]. Secondly, for velocity skewed flow, an additional streaming mechanism is introduced as a result of shedding of vortices of unequal strength in the successive wave half cycles [Davies and Villaret, 1999]. This additional mechanism contributes in onshore direction near the bed and in offshore direction higher up in the boundary layer. However, the most drastic change is the offshore directed contribution to waveaveraged sediment transport that results from asymmetrical vortex shedding [Van der Werf et al., 2007]: the strongest vortex, active around on to offshore flow reversal, produces a large near bed offshore flux and an increased suspension cloud, transported offshore during the offshore phase of the wave. Modeling the water and sediment dynamics above rippled beds requires a 2DV modeling 
approach [Van der Werf et al., 2008]. So far, most research on sediment transport above ripples has been carried out in tunnels and does therefore not include free surface effects. How progressive wave streaming and horizontal advection effects influence net transport over rippled beds is still an open question.

\subsubsection{Horizontal bed assumption \& steady, uniform wave assumption}

The models applied in this study assume that the bed level difference over a wave length is negligible compared to the wave length (horizontal bed). Together with neglecting energy loss during propagation, this forms the basis for the steady, uniform wave assumption. The assumption of steady, uniform waves made it possible to consider the horizontally non-uniform flow beneath progressive waves in a $1 \mathrm{DV}$ framework. In case of sloping beds, waves are not uniform: for perpendicular incident waves, period averaged wave characteristics and water levels will change during propagation due to shoaling, water level set-down, depth-induced breaking and subsequent water level set-up.

An important effect of slopes is therefore that the mean pressure gradient and the magnitude (and profile shape) of the return current are subjected to changes. This difference is especially clear when comparing profiles under breaking and under non-breaking waves: Reniers et al. [2004] e.g. found from measurements and period-averaged modeling that within the surfzone the maximum return flow velocities occur in the lower part of the water column, while in the shoaling zone the maximum return flow velocities occur closer to the water surface and are generally much smaller. However, also before breaking the strength of the return current was found to increase with reducing water depth. In the validation cases of chapter 3 (figure 7), the influence of the return current on sediment transport was found to be rather small. Although the Schretlen [2012] experiments do consider relative large waves, it might be unfounded to assume small return current influence on transport for all non-breaking waves. Note that the modeling tools itself have no restrictions in dealing with situations with increased pressure gradient and return current. However, to simulate situations with increasing return current, predictions of pressure gradient or return current will be needed to properly force the boundary layer model.

For increasing steepness, the slope-induced changes in the wave characteristics will also affect the adequacy of the $1 \mathrm{DV}$ approach through the transformation $\partial . . / \partial x=-c^{-1} \partial . . / \partial t$. Next, slope effects will also start to influence flow and transport directly. Regarding the flow, slope effects induce an uphill period-averaged current inside the boundary layer [Fuhrman et al., 2009a]. This slope related streaming is the result from convergence/divergence differences between the uphill and downhill water motion. Although estimates of Fuhrman et al. [2009b] show that for realistic slopes the slope related streaming will be small compared to velocity skewness streaming and progressive wave streaming individually, it might play a role in the delicate balance of the joint streaming processes. Regarding sediment transport, slopes evidently also have a hampering effect on uphill transport, against the direction of gravitational acceleration (and an opposite effect on downhill transport). 
To determine how flow and transport behave under the summation of all these effects, further research will be needed. Firstly, we recommend to give a better account for the mean pressure gradient. This could be achieved already within the assumption of a (locally) horizontal bed and (locally) uniform waves. A first option is to couple the model to a flow model on infra-wave time scale that predicts water level gradients and pressure gradients. Alternatively, a pressure gradient and return flow estimation / computation could be incorporating in the model itself. Note that we lost this functionality by leaving the 'whole water column approach' of the original PSM model (see introduction), that included a Stokes' drift and streaming compensating return current. Zhang et al. [2011] included shoaling and breaking effects on the mean pressure gradient in a first order boundary layer model. Including their approach in our model might enable determination of the role of the mean pressure gradient relative to progressive wave effects and wave shape effects for various wave conditions. Further in-depth research on boundary layer flow and transport for sloping beds and non-uniform waves presumably requires a 2DV modeling approach, combining both intra wave turbulence modeling and wave propagation prediction.

\subsubsection{Uniform sediment assumption}

Within this study, sediment is assumed to be uniform and the median grain size $d_{50}$ is used as representative value. This ignores that realistic sand samples are a mixture of sediments with different sizes. Hassan and Ribberink [2005] found from sheet-flow experiments in velocity skewed oscillatory flow that mixtures with a large fraction of fine grains generally show a reduced total net sand transport rate compared to mixtures with the same $d_{50}$ but smaller fractions of fine grains. This can be understood from the phase-lag effect: a larger fraction of fine sand means a larger amount of sediment still in suspension at flow reversal and a larger amount of sediment transported in offshore direction. However, Hassan and Ribberink [2005] also found that the transport rates of individual size fractions in a mixture are strongly influenced by the presence of the other fractions. Fine particles in sand mixtures are relatively less transported than those particles in nearly uniform sand. On the other hand, the contribution of coarse fractions in a mixture to the total net transport was found larger than expected from their volume fraction in the mixture. This was explained from vertical segregation of grain sizes in the upper layer of the bed. At the end of the experiments, a coarse surface layer was found on top a relatively fine sub-layer. This provides a relatively large flow exposure for the coarser grains, while it hides the fine grains. Hassan [2003] provides some results from intra wave boundary layer modeling with a multi fraction approach. Simply splitting up the sediment in fractions and applying the pick-up and diffusion computations on each separate fraction did not show an improvement of the total transport predictions over the uniform modeling approach. Only with a number of corrections, improved results were obtained. Firstly, a fraction approach was applied in which the coarsest fractions were assumed absent in the suspension. Secondly, a linear correction was applied on the transport of fractions with $d_{\mathrm{m} \text {,fraction }}>d_{50 \text {, mixture }}$ to give account for the increased exposure of the coarse sand. Considering the potential role of sieve curve differences in explaining the differences between the two validation data sets of medium sized sediment in flumes (1-4 and 10-13 in Table 3.1, chapter 3), further elaboration of the multi-fraction approach and suggested improvements might be informative. Here we refer to the fact that the 
medium sized sediment conditions of Schretlen [2012] that contain a larger fraction of coarse sediment $\left(d_{90}=0.42 \mathrm{~mm}\right)$, showed smaller net transport rates than Dohmen-Janssen and Hanes [2002] $\left(d_{90}=0.28 \mathrm{~mm}\right)$, and were, unlike Dohmen-Janssen and Hanes [2002], slightly overpredicted by the model. However, it should be noted that in flumes effects of fine size fractions may appear different from those described above, because the phase lag effect for fine sand is counteracted by progressive wave streaming and advection effects.

\subsubsection{Acceleration skewness}

The flume experiments used in the validation of the BL2-SED and 2PH model (Table 3.1, chapter 3, condition 1-13) are predominantly velocity skewed. Also the numerical tests consider velocity skewness only. A relevant question is what the effects are of free surface effects for progressive surface waves with increasing acceleration skewness towards the surfzone. For (theoretical) purely acceleration skewed waves, it may be expected that progressive wave streaming and horizontal advection effects will contribute to onshore transport, because the mechanisms are basically the same as for velocity skewed waves. However, contrary to velocity skewed waves, for acceleration skewed waves shear-stress asymmetry and phase-lag effects do also contribute to onshore transport [Van $\operatorname{der} A, 2010]$. As a results, there will be no compensation of offshore transport from phase lag effects by onshore transport due to progressive wave effects, but two mechanisms both contributing to onshore transport. A gradually increasing acceleration skewness of a progressive wave may therefore be expected to result in increasing transport in onshore direction. We recommend further research to quantify the progressive wave effects for acceleration skewed waves. Although model validation and application were mostly limited to velocity skewed conditions, there is no modelrelated restriction to apply the model for acceleration skewed waves. This is supported by the modeldata comparison for flow velocities (in chapter 2.3) and sediment transport (by Ruessink et al. [2009]) for acceleration skewed oscillatory flow. Finally, note that increased acceleration skewness occurs close to wave breaking. It may therefore coincide with many other effects. We already discussed the increasing importance of return currents close to the breaker point. At the same time, phase-lag effects and advection effects may decrease, because around the breaker line sand is usually coarser than further offshore.

\subsubsection{Wave irregularity and wave breaking}

Within this study, model validation and application has been limited to regular, non-breaking waves. In prototype situation, waves are often irregular and will break near the shore. Wave grouping / randomness and wave breaking introduce many additional flow and transport processes both in and above the wave boundary layer. Concerning transport, an interesting boundary layer phenomenon is the "pumping" of sediment during sequences of high waves [Vincent and Hanes, 2002], [Holmedal et al., 2004]. For breaking waves, Scott et al. [2009] pointed at the importance of the timing of breaker-induced turbulence reaching the wave boundary layer. The present model can be a useful tool to investigate the role of streaming and advection effects for "pumping" irregular waves and wave groups. Next, a model investigation on the differences in transport between regular and irregular waves will certainly contribute to the improvement of practical sediment transport formula. 
The influence of breaking on sediment transport is less suitable to be investigated with the modeling concepts adopted in this study (note that Scott et al. [2009] used the two-phase boundary layer model of Hsu and Hanes [2004]), because the complexity of breaking related processes outside the wave boundary layer are hard to be schematized in the forcing of the wave boundary layer model.

\subsection{TOWARDS MORPHODYNAMIC MODELING}

This section reflects on the role of the intra wave boundary layer models within the present study and the various ways our results could be employed in morphodynamic modeling.

\subsubsection{Through sediment transport formulas}

Within this study, process-based models have been mainly applied to obtain additional insights in physical mechanisms behind wave-induced sediment transport. The models were used as tool to interpret observations, to determine the relative importance of various processes, and subsequently to develop parameterizations of the various processes in aid of practical sediment transport formulas. In this approach, the numerical model functions as a supplier to the sediment transport formulas which can subsequently be applied in morphodynamic computations (see Figure 5.1). This approach is consistent with the research methodology applied in many more preceding sediment transport (PhD-) studies, e.g. Al-Salem [1993] on effects of velocity skewness, Dohmen-Janssen [1999] on grain size influence, Hassan [2003] on effects of graded sediment, Van der Werf [2006] on sediment transport over ripples, $\operatorname{Van} \operatorname{der} A$ [2010] on acceleration skewness effects (all for wave-induced transport). All these studies investigate a specific process or phenomenon in isolation, and provide parameterizations to include the considered aspect in sediment transport formulas and roughness expressions, which than form the connecting element between the detailed (mostly experimental but also numerical) process studies and the larger scale morphodynamic modeling. The advance of this approach is that it provides a clear understanding of the physics involved, that it underpins the sediment transport formulas with a clear physical basis and finally leads to a tool that covers a wide range of processes, conditions and transport regimes. In line with this approach, a part of the results of the present study has been added already to the sediment transport formula under ongoing development [Van der A et al., 2011] (including streaming and a preliminary implementation of the advection effects), and this formula has been applied already in the framework of a morphodynamic modeling system by Van der Werf et al. [2012]. (A complete description of the sediment transport formula including free surface effects is given by Van $\operatorname{der} A$ et al. [n.d.]).

\subsubsection{Through direct application of the process-based models}

For experimental studies, the approach described above is clearly the most practical way to apply the results in morphodynamic modeling. However, alternatively, intra-wave boundary layer models might be also directly applicable in morphodynamic modeling. What would be the advantages, necessities or practical limitations for that? 


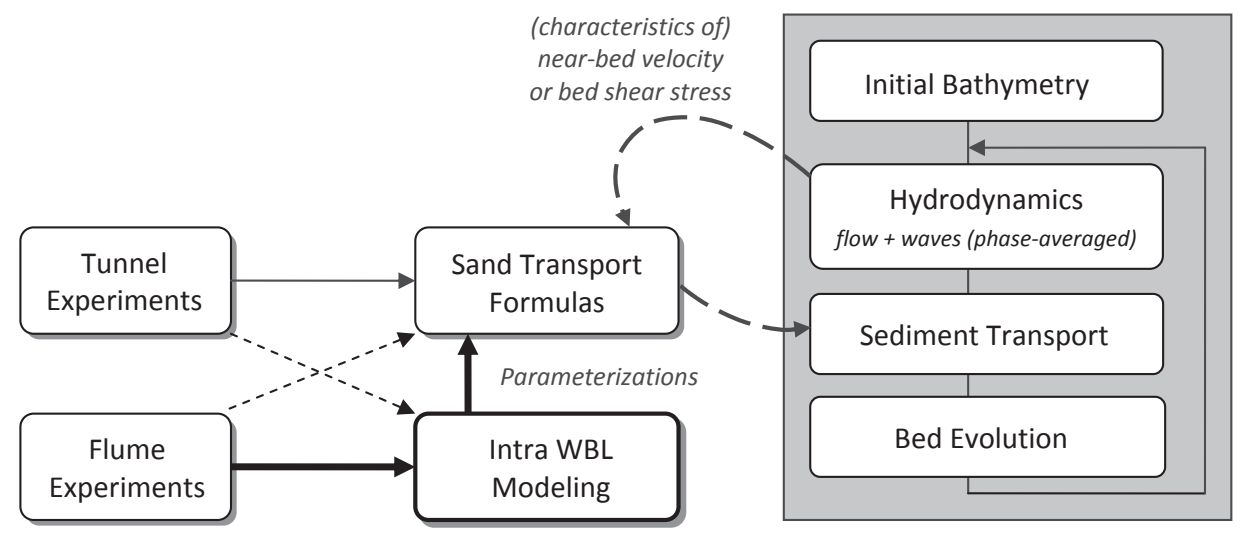

Figure 5.1: Relation between experiments, intra wave boundary layer modeling, sand transport formulas and morphological modeling. Thick line: present project; dashed ellipse: way to involve the results of the present study in morphodynamic modeling.

The main advantage of direct application of the numerical model is the possibility to avoid a part of the simplifications involved in parameterizations. Next, in principle a numerical model is better able to deal with combinations of mechanisms. Concerning e.g. wave shape effects, the transport formulas have been adapted and tuned for effects of both velocity skewness and acceleration skewness, but their effectiveness for waves with combined velocity and acceleration skewness still needs further validation. The numerical model is actually validated for the underlying processes of advection and diffusion and can be applied for any wave shape.

To directly apply the BL2-SED model of chapter 3 in morphodynamic computations, input is required for (near bed free stream) velocities. This information needs to be obtained from the hydrodynamic part of the modeling system. In present practice, often the hydrodynamic part consists of a flow module to solve the infra - wave dynamics (e.g. currents, long waves) and a wave module to provide wave properties and wave-averaged forces, see e.g. Lesser et al. [2004] and Ruessink et al. [2007]. In many engineering applications, (linear and period-averaged) wave energy models are used as wave module. Subsequently, the thus obtained wave

height is translated into near bed orbital velocities or bed shear stresses using non-linear theories (e.g. Rienecker and Fenton [1981]) (although a trend is present to develop and apply empirical expressions for this step [Elfrink et al., 2006], [Abreu et al., 2010], [Ruessink et al., 2012]). Next, the (characteristics of the) near bed orbital velocities and current velocities are used as input for a sediment transport formula.

For direct application of the intra WBL model, the same velocity information could be used. However, this introduces an unbalance between the various part of the modeling system: a rather detailed sediment transport module is than fed from a rather crude method for near bed velocity 
predictions. The increasing interest in the influence of the wave shape on sediment transport and morphodynamic has recently led to various studies using more detailed wave models in combination with sediment transport formula ([Hoefel and Elgar, 2003], [Lescinski and Özkan-Haller, 2004], [Long et al., 2006], [Wenneker et al., 2011]). Coupling of these wave models to an intra WBL model like BL2-SED is a consistent next step in which the development of wave shape predictors and sediment transport models keep pace. Note that within such implementations there is no need to compute transport on the same spatial and temporal grid as hydrodynamics. Concerning time, transport can be computed every morphological time step (e.g. determined from the "activity" of the bed). Concerning space, interpolation can be applied between locations on a coarser grid. The computational effort could be further reduced by the use of look-up tables, prepared in advance from a large number of intra WBL computations. Another option might be to derive a sediment transport formula from these synthetic intra WBL model data, e.g. through Generic Programming, as applied for vegetation roughness by Baptist et al. [2007].

Except for the computational effort, a disadvantage of direct application of the present BL2-SED in a morphodynamic modeling system is the limitation to the sheet-flow regime only. On the other hand, present sediment transport formulas allow for a smooth transition between the rippled and sheet-flow regime as function of $\Psi$ or $\theta$. Furthermore, it needs to be considered that even the more detailed process based models do only partly account for the complex physics of sediment transport in practice.

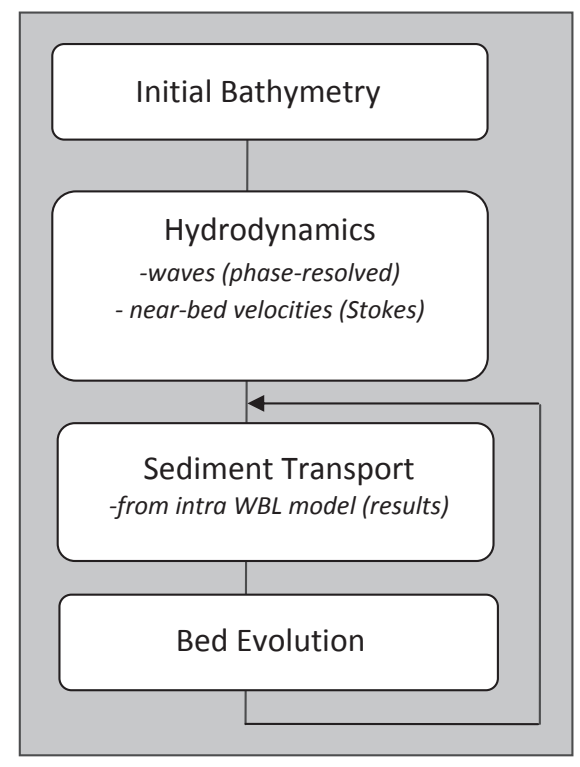

Figure 5.2: Structure of the simple morphodynamic model of section 5.3. 


\subsection{MORPHODYNAMIC APPLICATION: SANDBAR MIGRATION}

In the following example, we compute the morphological development of a cross-shore profile using the BL2-SED model of chapter 3. The aim of this exercise is to illustrate the potential implications of either or not considering free surface effects for predictions of cross-shore morphology. Simultaneously, we illustrate how our process-based model could be applied in the context of a simple morphodynamic model.

We consider a cross-shore profile with a single sand bar around $200 \mathrm{~m}$ offshore (Figure 5.3, panel a). The profile is affected by three days of wave action of perpendicular incident, steady waves (period $T=5.0 \mathrm{~s}$, height $H=0.8 \mathrm{~m}$ at $450 \mathrm{~m}$ offshore where water depth $h=6.3 \mathrm{~m}$ ). The chosen profile and wave conditions are based on the situation on 24-26 September 1994 near Duck, NC, USA [Gallagher et al., 1998], where onshore bar migration was observed. For these conditions, the nearbed flow in the bar area will be large enough to generate sheet-flow, while the waves will not break at the bar and the return current influence will be limited.

The first step in our example computation is to calculate the wave height along the profile with a shoaling computation starting at $6.3 \mathrm{~m}$ water depth. For simplicity, energy loss from bottom friction is neglected and waves are assumed to break as soon as the wave height / water depth ratio exceeds 0.65 (Figure 5.3, around $x=-80 \mathrm{~m}$ ). In the next step, the wave-related near-bed velocity is calculated along the profile from the wave period, wave height and water depth using $2^{\text {nd }}$ order Stokes theory (see equation 8, chapter 2). For a selection of 24 cross-shore locations between $x=-450$ and $x=-100$ $\mathrm{m}$, simulations are carried out with the intra WBL using the calculated near-bed velocity signal as forcing. These model runs result in values for the wave-averaged sediment transport rates $\left\langle q_{\mathrm{s}}\right\rangle$ at these locations. The results are interpolated (using splines and a fine $x$-grid) to obtain sediment transport rates along the profile from 450 till $100 \mathrm{~m}$ offshore (Figure 5.3, panel b, the dots are model results). Subsequently, profile changes are calculated from the convergence / divergence of sediment transport during time intervals of $1 / 2$ an hour. After updating the bed profile, new transport rates are determined for the selected cross-shore locations. However, rather than running new computations / simulations for hydrodynamics and sediment transport, this is done using the old simulation results: because energy loss from friction was neglected and no memory for wave shape deformation is present in the used wave theory, every water depth is connected to a single wave height and wave shape and therefore to a single results for the transport rate. Hence, the transport rates belonging to the new bed levels / water depths can be determined from interpolation between the earlier computed $\left.<q_{\mathrm{s}}\right\rangle$-values. In this way (see Figure 5.2), profile changes are computed till three days have passed (144 time steps). We carry out this procedure using the intra WBL model both with (BL2) and without (BL1) free surface effects. The resulting bed levels around the sandbar are shown in (Figure 5.3 , panel c).

We conclude from Figure 5.3 that either or not including progressive wave effects in morphodynamic predictions can result in large differences in the predicted sandbar migration. In this example, the difference in predicted migration speed of the sandbar crest with and without 
progressive wave effects is a factor 2. Note that for finer sized sand, e.g. $d_{50}=0.15 \mathrm{~mm}$, larger differences and even opposite migration directions may be expected, with offshore migration when progressive wave effects are neglected. Notwithstanding the simplifications in this model and the absence of validation with measurements, we believe the large difference in migration rate is an important observation. As discussed before, present day morphodynamic models do not or only limitedly account for free surface effects. At the same time they tend to under predict onshore transport in accreting conditions (see e.g. Gallagher et al. [1998], Van Rijn et al. [2011]). Together, these issues further underline the necessity to properly accounting for free surface effects in morphodynamic models.

(a)

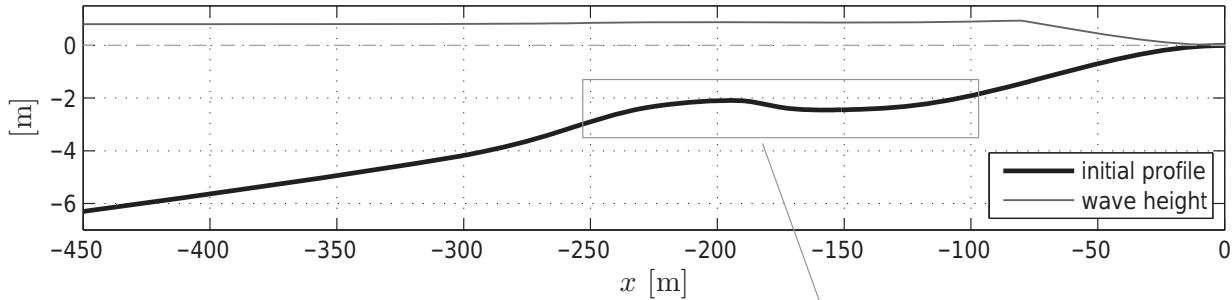

(b)

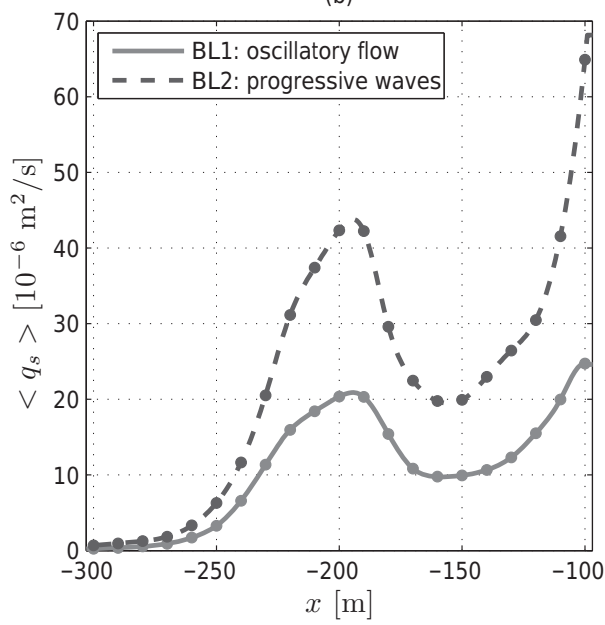

(c)

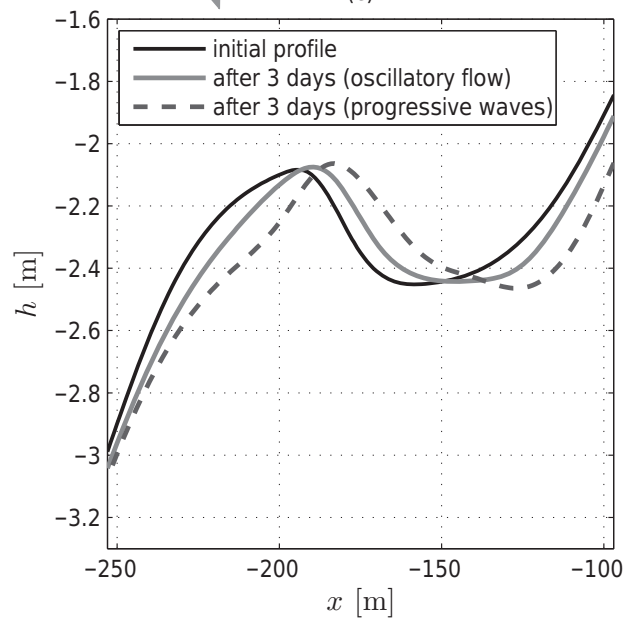

Figure 5.3: Morphodynamic example: computation of the development of a cross-shore profile using the process-based numerical model of chapter 3, either with (BL2) or without (BL1) progressive wave effects. (a) initial bed level and wave height; (b) wave averaged sediment transport rates $\left\langle q_{\mathrm{s}}\right\rangle$ during the first time step; (c) resulting bed levels around the sandbar after 3 days, all plotted against the cross-shore position. Condition: $T=5.0 \mathrm{~s}, H=0.8 \mathrm{~m}$ at $6.3 \mathrm{~m}$ water depth, $d_{50}=0.20 \mathrm{~mm}$ 


\subsection{MORPHODYNAMIC APPLICATION: SAND WAVES}

The significance of the insight that the advective terms may largely influence net sediment transport is not restricted to wave-induced sand transport only. Here we discuss an application in the wider field of seabed morphology, namely the occurrence of sand waves. Sand waves are rhythmic bed forms at sandy beds of tidal seas. There formation is explained from self-organization due to interaction between the sandy seabed and the tidal flow [Hulscher, 1996]. Initial small amplitude perturbations cause flow contraction and therefore pressure reduction above the crest of the perturbations. This happens both during flow with (positive) and against (negative) the direction of tidal wave propagation. The pressure difference generates a small tide averaged near bed current from both sides of the perturbation towards the crest. The sand transported by this current feeds the perturbation and strengthens the mechanism. Sand waves are found at many locations in the North Sea. However, the prevalence and the height of sand waves has been found to diminish with decreasing sand bed grain size [McCave, 1971], which is attributed to the increasing role of suspended sediment. Indeed, recent numerical sand wave simulations of Borsje et al. [n.d.] (extension of Borsje et al. [2011]) including suspended sediment transport, show a growth reduction and even negative growth for sand waves in case of dominant suspended sediment transport. With the insights of the present study, we are now able to explain the damping mechanism and the absence of sand waves in parts of the North sea with finer sands.

Like the additional onshore transport under (short) surface waves, the damping effect of suspended load transport on sand wave formation can be explained from slight differences in sediment concentration between both halves of the cyclic flow forcing. Both during positive and negative flow, the absolute horizontal velocities $u$ and the sediment concentrations $c$ are slightly higher above the crest of the sand wave compared to the trough. This will lead to a divergence in sediment advection $c u$ during the uphill motion and a convergence during the downhill motion. This induces a slight modulation of the sediment concentration above the flank of a sand wave with the period of the tide. As a consequence, the suspended load transport rate at the flank of a sand wave is larger during the downhill motion than during the uphill motion.

Figure 5.4 shows the various contributions to the tide-averaged suspended load transport rate $\langle\mathrm{cu}>$ from 2DV numerical simulation of $M_{2}$-tidal flow over a number of sand waves (tidal amplitude $\hat{u}_{\mathrm{M} 2}=$ $0.65 \mathrm{~m} / \mathrm{s}$, sand waves length $L_{\mathrm{sw}}=600 \mathrm{~m}$, original height $H_{\mathrm{sw}}=1.0 \mathrm{~m}, d_{50}=0.2 \mathrm{~mm}$, mean water $h$ $=25 \mathrm{~m})$. The tide-averaged current $\left(U_{\mathrm{M} 0}\right)$ induces a convergence of suspended load transport at the sand wave crest and hence growth of the sand wave (Figure 5.4B). However, the $u$ and $c$ fluctuations with the frequency of the tide produce a flattening contribution $\left(<_{\mathrm{C}_{2}} u_{\mathrm{M} 2}>\right.$, Figure $\left.5.4 \mathrm{C}\right)$, which is clearly dominant in this case (compare panel $\mathrm{C}$ with $\mathrm{A}$ ).

Interestingly, both for flow over sand waves and sheet-flow under free surface waves, the (sand or surface wave-related) period averaged represents only a part of the dynamics additional to the horizontally uniform situation. However, where for waves over a flat bed current and advection convergence/divergence effects both contribute in the same way, these processes counteract in the 
case of flow over a wavy bed. From an engineering point of view, the importance of accounting for advection effects is therefore even larger in the sand wave case.
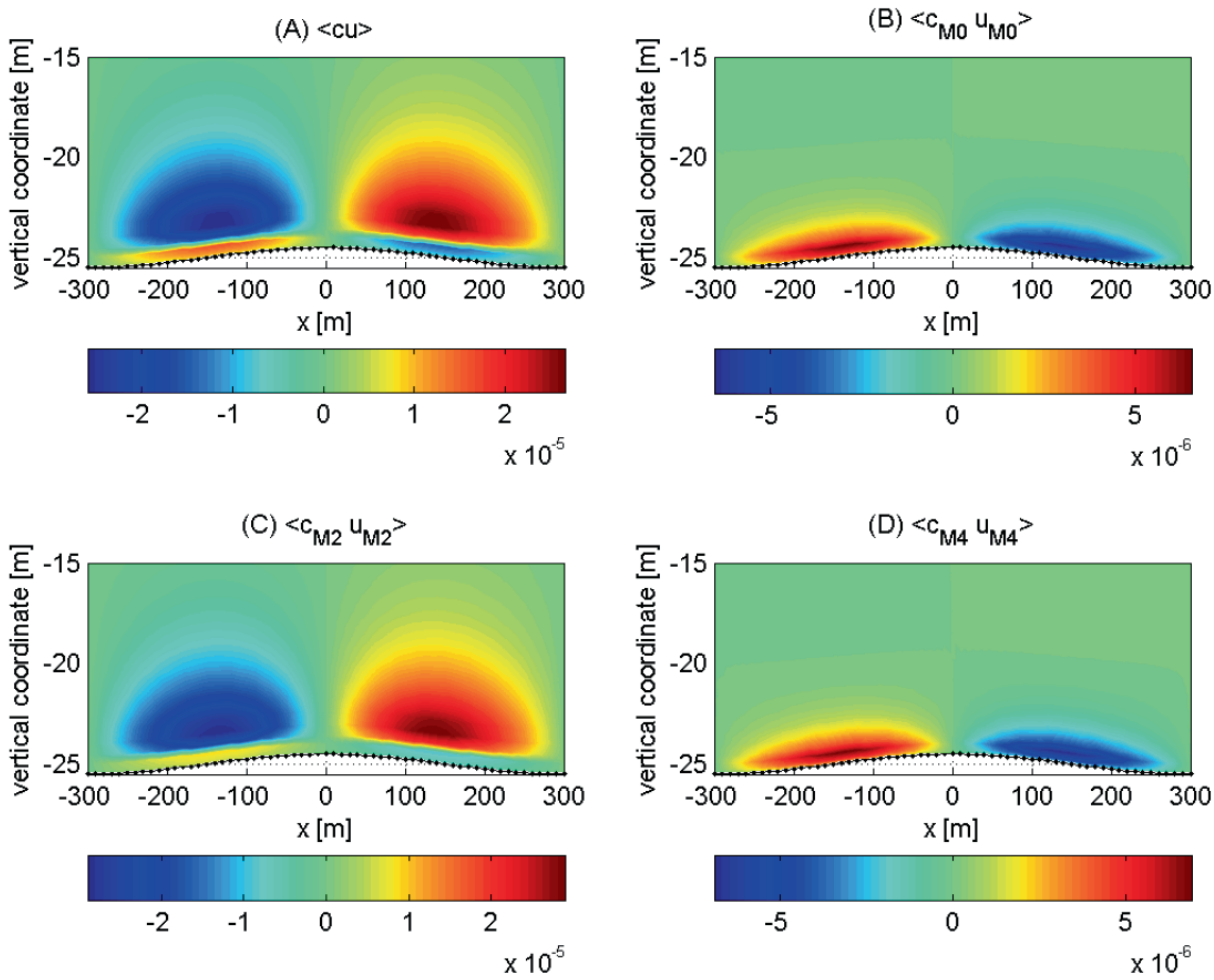

Figure 5.4: Tide-averaged suspended sediment flux $\langle\mathrm{Cu}\rangle\left[\mathrm{kg} \mathrm{s}^{-1} \mathrm{~m}^{-2}\right]$ above a sand wave, and the contributions to it from the mean $\left\langle c_{\mathrm{M} 0} u_{\mathrm{M} 0}>\right.$ (B), first $<c_{\mathrm{M} 2} u_{\mathrm{M} 2}>$ (C) and second $<c_{\mathrm{M} 4} u_{\mathrm{M} 4}>$ (D) harmonic components of concentration $c$ and horizontal velocity $u$. Positive fluxes (red) are directed to the right. Conditions: $\hat{u}_{\mathrm{M} 2}=0.65$ $\mathrm{m} / \mathrm{s}, L_{\mathrm{sw}}=600 \mathrm{~m}, H_{\mathrm{sw}}=1.0 \mathrm{~m}, d_{50}=0.2 \mathrm{~mm}, h=25 \mathrm{~m}$; figure from Borsje et al. [n.d.] 


\section{CONCLUSIONS AND RECOMMENDATIONS}

The objective of this study was to develop a detailed understanding of the effects of progressive wave streaming on boundary layer flow and sheet-flow sand transport processes beneath surface waves for realistic wave and bed conditions by development, validation and application of numerical models for wave-induced sediment transport. The objective has been elaborated in a number of research questions. This chapter summarizes the answers on these research questions. Next, a number of recommendations for further research are listed.

\subsection{CONCLUSIONS}

RQ1: How can we develop process-based numerical tools to investigate the effects of progressive wave streaming on flow, transport and detailed sheet-flow layer processes for realistic wave and bed conditions?

Progressive wave streaming is the result of vertical advection of horizontal momentum. Therefore, the most important element to be included in any model to investigate wave-induced streaming is the vertical component of the orbital velocity. Inside the wave boundary layer, this velocity component is very small and the influence of vertical momentum advection on the time-dependent horizontal fluid motion is negligible. For that reason, it is often neglected in wave boundary layer models. However, this is not appropriate when the wave-averaged motion is considered. Next to vertical advection, also differences in turbulence between the on- and offshore phase of the wave are fundamental for good prediction of the wave-averaged current. Therefore, also the turbulence model - including the effect of sediment on the carrier flow turbulence - is of key importance for our modeling tools.

RQ2: How important is progressive wave streaming for the turbulent boundary layer flow above a fixed rough bed relative to other current generating processes, especially wave shape streaming? How do changes in wave and bed conditions affect the balance between these processes?

To answer this question, we developed a numerical boundary layer model including progressive wave effects: a 1DV-RANS-numerical boundary layer model with $k-\varepsilon$ turbulence closure has been extended with horizontal and vertical advection of momentum and turbulence properties. The model has been validated with good agreement against detailed experimental data on different types of wave boundary layer flow.

A generic analysis of the balance between onshore directed progressive wave streaming and offshore directed wave shape streaming for changing wave and bed conditions has been carried out by studying their separate contributions to the total non-dimensional streaming velocity $U_{0} c / \hat{u}_{1}{ }^{2}$. For turbulent flow, $U_{0} c / \hat{u}_{1}^{2}$ at the top of the wave boundary layer is a function of relative water depth $k h$ and bed roughness parameter $A / k_{\mathrm{N}}$ only. At relative deep water (large $k h$ ) the non-dimensional 
streaming velocity $U_{0} c / \hat{u}_{1}{ }^{2}$ is completely determined by the onshore directed progressive wave streaming. For decreasing relative water depth $(k h)$, the normalized progressive wave streaming stays nearly constant, but the relative importance of the wave shape effect increases and even becomes dominant. This means that the direction of the current inside the wave boundary layer will reverse from onshore to offshore directed during wave propagation towards the shore. The effect of bed roughness on the balance between the streaming processes is less distinct. For increasing relative bed roughness (decreasing $A / k_{\mathrm{N}}$ ), we found slightly increased contributions from onshore progressive wave streaming.

The model results for $2^{\text {nd }}$ order Stokes waves have been parameterized in an expression for the streaming velocities at the top of the boundary layer as function of $k h$ and $A / k_{\mathrm{N}}$ :

$$
\frac{U_{0} / \hat{u}_{1}}{\hat{u}_{1} / c}=0.345+0.7\left(\frac{A}{k_{N}}\right)^{-0.9}-\frac{0.25}{\sinh ^{2}(k h)}
$$

Progressive wave streaming also affects the bed shear stress. Our numerical results confirm earlier analytical estimates of the mean bed shear stress under sinusoidal waves. In addition, we found that this estimate can also be applied in the presence of non-linear wave shapes or strong wave-averaged pressure gradients.

RQ3: To what extent is progressive wave streaming important for sheet-flow transport of fine and medium sized sand, relative to other transport generating effects of the free surface wave? How do changes in wave and bed conditions affect the role of these processes?

Firstly, the effect of progressive wave streaming on wave-averaged sediment transport rates has been explored using the hydrodynamic model results for bed shear stress without and with progressive wave streaming in combination with a sediment transport formula. For the investigated cases (medium sized grains), this resulted in a progressive wave streaming induced increase of transport rates with $40 \%$ to $100 \%$ (chapter 2 ).

Next, RQ3 is investigated in more detail with the hydrodynamic model of chapter 2 extended with formulations describing the pick-up, the advective and diffusive transport and the turbulence damping effects of suspended sediment (chapter 3). This approach made it possible to differentiate between the contribution to sediment transport from progressive wave streaming and from other advection processes, and to determine whether progressive wave streaming is the full explanation of the observed increased onshore transport rates in flumes compared to tunnels. The results show that the onshore progressive wave streaming indeed contributes largely to increased onshore transport rates in flumes. However, especially for fine grains, also other advection processes are important. In particular convergence and divergence in horizontal advection of sediment in the non-uniform flow field beneath surface waves are found to influence fine sand transport significantly. These mechanisms amplify respectively reduce the maximum (suspension) concentration during onshore respectively offshore motion, causing an onshore directed contribution to the wave-averaged 
sediment transport. Quantification is given in Figure 6.1, showing simulated net transport rates $q_{\mathrm{s}}$ of medium $(0.25 \mathrm{~mm})$ and fine $(0.14 \mathrm{~mm})$ sized sediments for $2^{\text {nd }}$ order Stokes waves with increasing energy. For velocity-skewed waves, the horizontal advection effects work against the phase-lag effect that caused the offshore transport of fine sands in velocity-skewed oscillatory flow.

Considering their large impact on sediment transport, we conclude that not only streaming but also horizontal advection effects should be considered in formulas of wave-induced sediment transport in morphodynamic modeling. We propose to incorporate this effect in transport formulas through a parameter describing the adaptation time of sediment concentrations to changes in the flow velocity. The crux is that under progressive waves, this adaptation time not only depends on the grain size, but also on the flow direction with or against wave propagation. The proposed parameter $T_{\mathrm{a}}$ (chapter 3 , (3.21)) covers the relevant characteristics of the physical process, yields transport rates comparable to the numerical model and is therefore a suitable parameter to be included in practical sand transport formulas.

RQ4: What is the influence of progressive wave effects on the erosion depth, sheet-flow layer thickness and the sediment flux taking place within the sheet-flow layer? How do these effects differ for various realistic grain sizes?
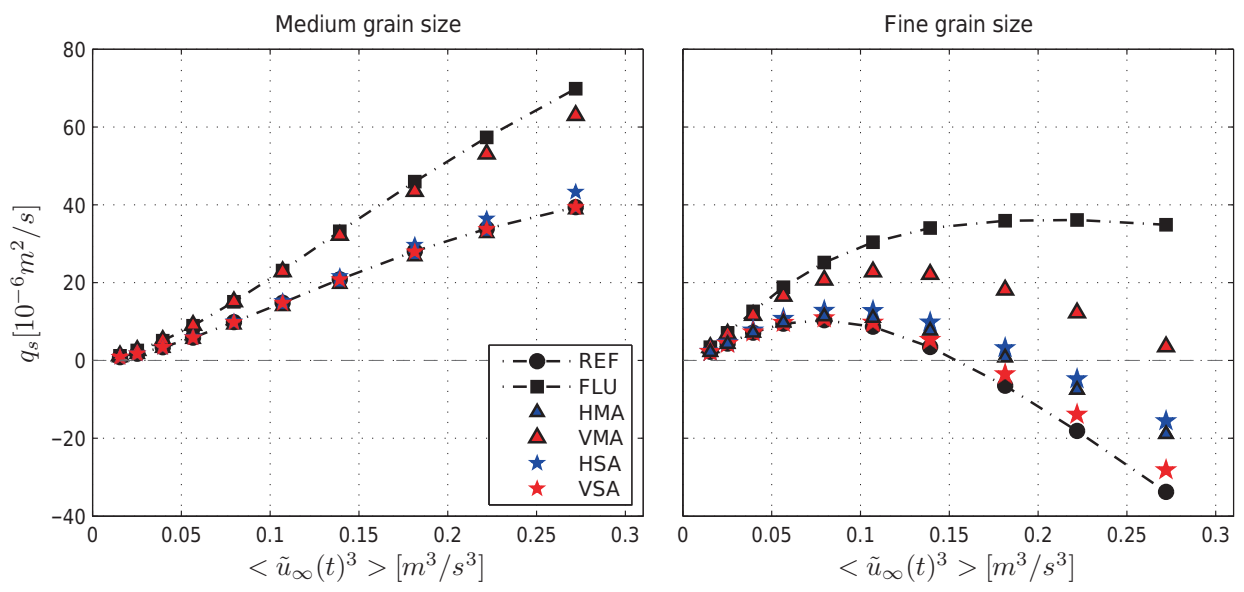

Figure 6.1: Net transport rates $q_{\mathrm{s}}$ of medium $(0.25 \mathrm{~mm})$ and fine $(0.14 \mathrm{~mm})$ sized sediments as function of third order velocity moment $\left\langle\tilde{u}_{\infty}{ }^{3}\right\rangle$ for $2^{\text {nd }}$ order Stokes waves with increasing energy. The figure shows results obtained with all advective processes switched on (FLU), all advective processes switched off (REF), and only terms related to a single advection process switched on. The letters in VMA, VSA, HSA and HMA denote: $\mathrm{V}=$ =ertical, $\mathrm{H}=$ horizontal, $\mathrm{M}=$ momentum, $\mathrm{S}=$ sediment and $\mathrm{A}=$ advection. Switching on VMA shows the influence of progressive wave streaming. 
In chapter 4, we have explored the possibilities to predict erosion depth and sediment fluxes and their dependency on grain size and streaming with a two-phase continuum model. To investigate RQ4, it appeared necessary to adapt the model formulations for grain - fluid turbulence interaction. With this adaptation, we extended the validity of the model towards finer grain sizes: the grain size dependent behavior of the model was largely improved and good reproductions were obtained of measured erosion depths for fine, medium and coarse sized sand beds. The large sensitivity of the results for this sub-model can be understood from increasing importance of fluid turbulence to support the grain motion with decreasing grain size.

From model-data comparison on concentration and velocity profiles, we concluded that the model is able to reproduce a number of important sheet-flow and boundary layer characteristics. This includes the tilting motion of the concentration profile during the wave. This is important, because this behavior reflects the typical structure of the sheet-flow layer, with respectively a pick-up layer with decreasing concentrations and an upper sheet-flow layer with increasing concentrations under increasing (absolute) flow velocities. As a result, the model is able to produce fluxes over the entire sheet-flow layer, also below the original bed level. Next, the model reproduced the phase lead of the near-bed flow, the thickness of the boundary layer and wave shape streaming and progressive wave streaming. The ability to predict streaming was also proven in the model reproduction of experimentally observed positive period-averaged velocities inside the sheet-flow layer and negative period-averaged velocities at slightly higher levels. Remaining inaccuracies mainly concern the behavior of concentration and velocity results for fine sand simulations during flow reversal and subsequent acceleration.

Comparison of sediment flux profiles for horizontally uniform oscillatory flow as in flow tunnels and horizontally non-uniform flow as under progressive waves, shows that fluxes inside the sheetflow layer increase in onshore direction under influence of progressive wave effects. This effect increases with decreasing grain size. For fine sand under velocity skewed waves, it is found that both the wave-related contribution (which is generally offshore inside the sheet-flow layer) and the current-related contribution (onshore inside the sheet-flow layer under velocity skewed waves) to the period-averaged sheet-flow sediment flux are affected. The results are consistent with trends for fine and medium sized sediment flux profiles observed from tunnel and flume experiments.

\subsection{RECOMMENDATIONS}

Based on our conclusion that both progressive wave streaming and non-linear horizontal advection effects are very important for wave-induced sediment transport, especially for fine sand, our first and most important recommendation is to consider these processes in morphodynamic studies. This requires that these processes are included in the sediment transport sub-models of the morphodynamic modeling systems, nowadays mostly sediment transport formulas.

We recommend implementation of progressive wave streaming and horizontal advection effects into sediment transport formulas through the parameterizations for these processes provided in this study 
(chapter 2, section 4.2.4, and chapter 3, section 5.1). We expect that an increased account for these sediment transport processes will contribute to improved morphological predictions, especially for accreting conditions under energetic, but non-breaking waves. For these conditions, present morphodynamic models tend to underpredict the morphological changes [Van Rijn et al., 2011], while the contribution of progressive wave effects to sediment transport will be relatively large.

For future research on wave-induced sediment transport with the intra wave boundary layer models of this study, we recommend:

- To improve the way these models account for the mean pressure gradient. This could be done by coupling the model to a flow model on infra-wave time scale or by including the prediction of the mean pressure gradient in the model itself.

- Further validation of the two-phase continuum model and additional research on the turbulence closure, especially its behavior around flow reversal. We believe improvements in this aspect can largely improve the overall predictive quality of the model. A further improved model will be a valuable instrument for further study and parameterization of sheet-flow layer processes.

Finally, this study was restricted to progressive wave effects for sheet-flow under regular, nonbreaking waves over flat, horizontal beds. Progressive wave effects in combination with nonuniform sand, ripples, bed slopes, acceleration skewed waves and wave-current combinations were not or only limitedly considered. Also the effects of wave irregularity and wave breaking on boundary layer flow and sediment transport have not been studied. Of the processes mentioned here, we especially recommend further research on wave boundary layer processes under combined velocity and acceleration skewed waves and under breaking waves. Both these situations occur shoreward of the domain considered in this study, where the morphological impact of the processes may be expected even stronger. Sand transport under breaking waves is one of the central topics of the new STW/EPSRC-funded research project SINBAD. 


\section{APPENDICES}

\section{APPENDIX A: BOUNDARY LAYER VELOCITIES (ANALYTICAL SOLUTIONS)}

(First referred to in section 1.2.1).

The vertical profiles of the amplitude $\hat{u}(z)$ and phase $\theta(z)$ of the horizontal component of the orbital velocity and the period-averaged current $U_{0}(z)$ in Figure 1.2 are described with:

$$
\begin{gathered}
\frac{\hat{u}(z)}{\hat{u}_{\infty}}=\sqrt{1+e^{-2 \beta z}-2 e^{-\beta z} \cos (\beta z)} \\
\theta(z)=\operatorname{atan}\left[\frac{e^{-\beta z} \sin (\beta z)}{1-e^{-\beta z} \cos (\beta z)}\right] \\
\frac{U_{0}(z)}{\hat{u}_{\infty}} / \frac{\hat{u}_{\infty}}{c_{p}}=\frac{1}{4}\left\{3+e^{-2 \beta z}-2 e^{-\beta z}[(\beta z-1) \sin (\beta z)+(\beta z+2) \cos (\beta z)]\right\}
\end{gathered}
$$

with $z$ the vertical level above the bed, $\hat{u}_{\infty}$ the horizontal velocity amplitude in the free stream, $c_{\mathrm{p}}$ the wave propagation velocity and $\beta$ the inverse of the Stokes length $\delta_{\mathrm{s}}=(2 v / \omega)^{1 / 2}$. These solutions have been obtained analytically for a layer with constant viscosity $v$ beneath a sinusoidal wave with angular frequency $\omega$. Equation (A.1) and (A.2) are first order solutions, equation (A.3) for the steady flow component is part of the second order solution. Expressions for streaming were firstly derived by Longuet-Higgins [1958]. The expressions (A.1), (A.2) and (A.3) are given by Svendsen [2006] See Svendsen [2006] section 10.1 and also Nielsen [1992] section 1.2 for details on the derivation. Because of the relevance of this material to the present study, we provide a summary of the derivations below.

Inside the WBL, the flow can be described in first order approximation with the equation and boundary conditions:

$$
\frac{\partial u}{\partial t}=\frac{\partial u_{\infty}}{\partial t}+v \frac{\partial^{2} u}{\partial z^{2}} ; \quad u(z=0)=0 ; \quad u(z \rightarrow \infty)=u_{\infty}=\hat{u}_{\infty} e^{i \psi}
$$

Here $\psi=\omega t-k x$ (which reduces to $\psi=\omega t$ in uniform oscillating flow). Using the 'defect velocity' $v=u-u_{\infty}$ and the $z$-independency of $u_{\infty}$, equation (A.4) can be transformed to:

$$
\frac{\partial v}{\partial t}=v \frac{\partial^{2} v}{\partial z^{2}} ; \quad v(z=0)=-\hat{u}_{\infty} e^{i \psi} ; \quad v(z \rightarrow \infty)=0
$$

Assuming solutions of the form $v=V(z) e^{i \psi}$, with $V(z)$ a complex amplitude, we arrive at: 


$$
i \omega V=v \frac{\partial^{2} V}{\partial z^{2}}
$$

which has solutions of the form

$$
V=C_{1} e^{\alpha z}+C_{2} e^{-\alpha z} ; \quad \text { with } \quad \alpha=(1+i) \sqrt{\frac{\omega}{2 v}}=(1+i) \beta
$$

From the boundary conditions for (A.5) it follows that $C_{1}=0$ and $C_{2}=-\hat{u}_{\infty}$. Therefore:

$$
u=\left(V+\hat{u}_{\infty}\right) e^{i \psi}=\left(-\hat{u}_{\infty} e^{-(1+i) \beta z}+\hat{u}_{\infty}\right) e^{i \psi}=\hat{u}_{\infty}\left(1-e^{-\beta z} e^{-i \beta z}\right) e^{i \psi}
$$

We arrive at (A.1) and (A.2) by taking the modulus and argument of the complex amplitude of $u$.

The first order solution for the vertical velocity $w$ (in non-uniform flow) can be derived from (A.8) using continuity and a boundary condition for $w$ at the bed:

$$
w=\int_{0}^{z}-\frac{\partial u}{\partial x} d z ; \quad w(z=0)=0
$$

and yields:

$$
w=i \frac{\hat{u}_{\infty} k}{\beta}\left\{\beta z e^{i \psi}+\frac{e^{-\beta z}}{\sqrt{2}} e^{i\left(\psi-\beta z-\frac{\pi}{4}\right)}-\frac{1}{\sqrt{2}} e^{i\left(\psi-\frac{\pi}{4}\right)}\right\}
$$

The expression for streaming, equation (A.3), is derived from the second order momentum balance after substitution of (the real parts of) $u$ and $w$ from equation (A.8) and (A.10). Hereby, the balance is averaged over the wave period and two times integrated over the vertical. The (non-zero terms of the) period-averaged balance and boundary conditions for $U_{0}$ read:

$$
\frac{\partial(\overline{u w})}{\partial z}=v \frac{\partial^{2} U_{0}}{\partial z^{2}} ; \quad U_{0}(z=0)=0 ; \quad \frac{\partial U_{0}}{\partial z}(z \rightarrow \infty)=0 ;
$$

Vertical integration using these boundary conditions results in equation (A.3). 


\section{APPENDIX B: EXPLORATION ON THE OCCURRENCE OF SHEET- FLOW}

(First referred to in section 1.2.2).

Our exploration of the occurrence of sheet-flow in front of the Dutch coast consists of three steps:

1. Analysis of 35 years of data of a wave buoy in front of the Dutch coast to obtain a schematized wave climate;

2. Construction of representative deep water wave conditions and translation of these conditions into wave heights and near bed velocities in the near shore area;

3. Determination of the depth from where sheet-flow may be expected.

Step (1): Figure B.1 shows a histogram of the joint occurrence of significant wave height $H_{\mathrm{s}}$ and mean wave period $T_{\mathrm{m} 02}$ in the three hour records of wave buoy YM6 (IJmuiden Munitiestortplaats, $30 \mathrm{~km}$ offshore at $21 \mathrm{~m}$ water depth). Based on this histogram, we derive functions to couple both the peak wave period and the probability of exceedance to the wave height at the wave buoy. The first relation is obtained from a linear fit through the mean $T_{\mathrm{m} 02}$ values per $H_{\mathrm{s}}$ bin, and the fact that for most spectra peak period $T_{\mathrm{p}} \approx 1.25 T_{\mathrm{m} 02}$. The second relation is obtained from fitting a Weibull distribution through the cumulative distribution of the records over $H_{\mathrm{s}}$. The resulting relations are:

$$
\bar{T}_{p}=1.25\left(a H_{s}+b\right) ; \quad \operatorname{Pr}\left\{H_{s}>H\right\}=\exp \left\{-\left(\frac{H-\gamma}{\beta}\right)^{\alpha}\right\}
$$

with $a=0.7845, b=3.4742, \alpha=1.1, \beta=0.9083$ and $\gamma=0.4190$. We use the coupled combinations of $H_{\mathrm{s}}, T_{\mathrm{p}}$ and $\operatorname{Pr}\left\{H_{\mathrm{s}}>H\right\}$ to schematize the wave climate. Neglecting potential effects of refraction and energy losses, we construct deep water conditions with $H_{0}=H_{\mathrm{s}} K_{\mathrm{sh}}{ }^{-1}$, where $H_{0}$ is the deep water wave height and $K_{\text {sh }}$ is the shoaling coefficient, see Table B.1 for some numerical values.

Step (2): Subsequently, the representative deep water conditions are translated to near shore wave heights using linear wave theory. Hereby, we consider shoaling and breaking, but neglect again energy losses from bottom friction and assume wave propagation perpendicular to the shore. The near bed velocities are subsequently calculated from the near shore wave heights using second order Stokes theory.

Step (3): From the near bed velocities, we determine for various grain sizes the depth from where sheet-flow may be expected. On the basis of laboratory observations, Wilson [1989] marked the transition to sheet-flow at Shields number $\theta=0.8$, where for oscillatory flow $\theta$ may be computed from the maximum near bed orbital velocity. Alternatively, the sheet-flow regime is delineated using the mobility number $\Psi$ [Soulsby, 1997], with sheet-flow for $\Psi>100-200$ and $\Psi=2 u_{r m s}^{2} /\left\{(s-1) g d_{50}\right\}$. Hereby $u_{\mathrm{rms}}$ is the root-mean-square of the velocity signal and $s=\rho_{\mathrm{s}} / \rho_{\mathrm{w}}$. 
In this exploration, we used the mobility number to determine the onset of sheet-flow: Figure 1.5 shows the line $\Psi=200$ for sand with median grain size $d_{50}=0.14$ and $0.25 \mathrm{~mm}$. Note that these median grain sizes are realistic grain sizes for the Dutch coast, and that grains near the breaker line are usually coarser than grains at deeper water.
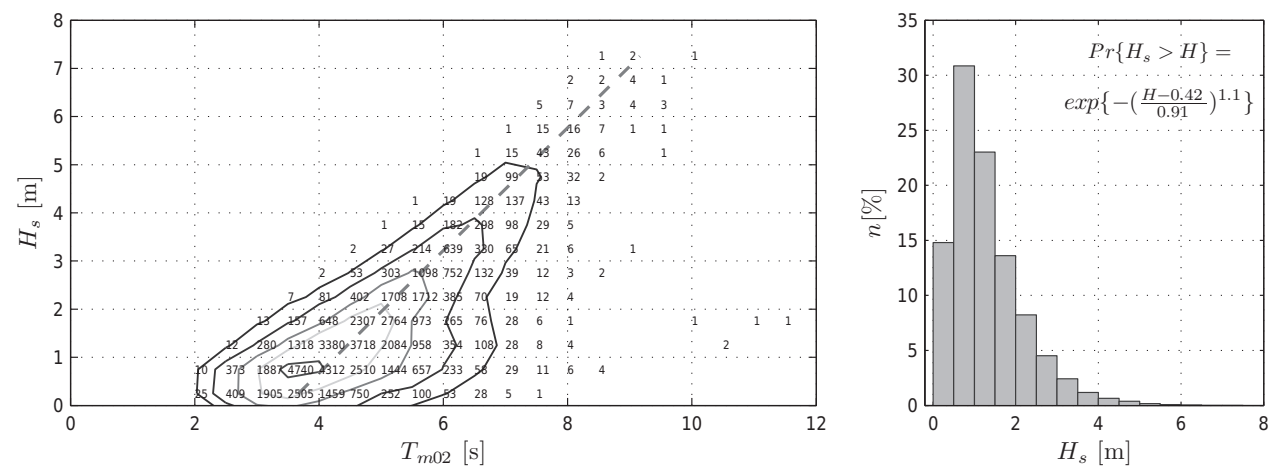

Figure B.1: Left) Histogram of the long-term, joint occurrence of significant wave height $H_{\mathrm{s}}$ and mean wave period $T_{\mathrm{m} 02}$ for the years 1976-2011 for RWS buoy YM6 (IJmuiden munitiestortplaats, data: waterbase.nl). Dashed line: fitted line through mean $T_{\mathrm{m} 02}$ values per $H_{\mathrm{s}}$ bin. Right) Histogram of $H_{\mathrm{s}}$ (same data), with $n$ the percentage of the total number of occurrences in the interval $\Delta H_{\mathrm{s}}=0.5 \mathrm{~m}$.

Table B.1: Schematized deep water wave characteristics in front of the Dutch coast as derived from wave buoy YM6.

\begin{tabular}{cccc}
\hline $\begin{array}{c}\text { Probability of } \\
\text { Exceedance (\%) }\end{array}$ & $\begin{array}{c}\text { Wave Height }(\mathrm{m}) \\
\text { at buoy }(h=21 \mathrm{~m})\end{array}$ & Wave Period (s) & $\begin{array}{c}\text { Deep Water } \\
\text { Wave Height }(\mathrm{m})\end{array}$ \\
\hline 50 & 1.1 & 5.4 & 1.1 \\
20 & 1.8 & 6.1 & 1.9 \\
10 & 2.4 & 6.6 & 2.5 \\
1 & 4.1 & 8.3 & 4.4 \\
\hline
\end{tabular}




\section{APPENDIX C: NUMERICAL SOLUTION METHOD ${ }^{4}$}

(Belonging to section 2.2.5).

The equations (2.1), (2.4) and (2.5) for momentum, turbulent kinetic energy and dissipation, are rewritten using (2.9) and (2.10) and discretized on a non-equidistant grid with staggered definition of the flow parameters: horizontal velocity and pressure are defined in the cell center, vertical velocity and turbulence properties at the cell interfaces, see Figure C.1.

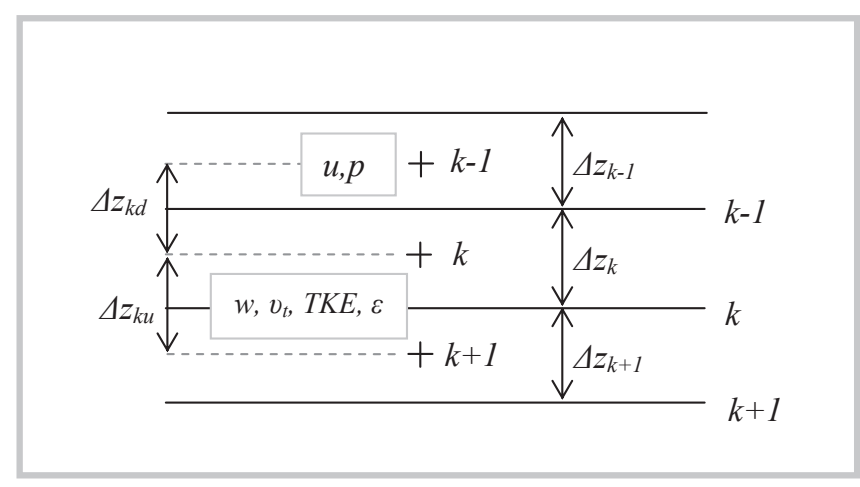

Figure C.1: Numerical grid (staggered), with layer and interface numbering and $\Delta z$-definitions. ${ }^{+}$) cell center; lines) cell interfaces.

Every time step the three balance equations are solved consecutively: at first the new velocity field is computed, subsequently the new turbulence properties are determined using the newly obtained velocity field. The momentum balance is solved in two steps: first a new velocity is predicted from the first order terms and an approximated non-linear horizontal advection term. This predicted horizontal velocity is used to predict the vertical velocity (continuity). Subsequently, predicted horizontal and vertical velocities are applied in the discretization of the non-linear advection terms in the corrector step. For the 'free' model formulation, the discretizations read:

Step 1:

$$
\begin{gathered}
\frac{\partial u}{\partial t}+u \frac{\partial u}{\partial x} \Rightarrow\left(1-\frac{u_{k}^{n}}{c}\right) \frac{u_{k}^{p}-u_{k}^{n}}{\Delta t} \\
-\frac{1}{\rho} \frac{\partial p}{\partial x} \Rightarrow\left(1-\frac{\tilde{u}_{\infty}^{n}}{c}\right) \frac{\tilde{u}_{\infty}^{n+1}-\tilde{u}_{\infty}^{n}}{\Delta t}
\end{gathered}
$$

\footnotetext{
${ }^{4}$ This appendix is extended compared to the appendix in the journal article.
} 


$$
\frac{\partial}{\partial z}\left(\left(v+v_{t}\right) \frac{\partial u}{\partial z}\right) \Rightarrow \frac{1}{\Delta z_{k}}\left(\left(v+v_{t, k-1}^{n}\right) \frac{u_{k-1}^{p}-u_{k}^{p}}{\Delta z_{k d}}-\left(v+v_{t, k}^{n}\right) \frac{u_{k}^{p}-u_{k+1}^{p}}{\Delta z_{k u}}\right)
$$

When $u^{p}$ has been solved, $w$ is predicted:

$$
w(z)=-\int_{0}^{z} \frac{\partial u}{\partial x} d z=\frac{1}{c} \int_{0}^{z} \frac{\partial u}{\partial t} d z \quad \Rightarrow \quad w_{k}^{p}=\frac{1}{c} \frac{1}{\Delta t}\left(\sum_{j=k+1}^{k \max }\left(u_{j}^{p}-u_{j}^{n}\right) \Delta z_{j}\right)
$$

Step 2:

$$
\begin{gathered}
\frac{\partial u}{\partial t}+u \frac{\partial u}{\partial x} \Rightarrow\left(1-\frac{u_{k}^{n}+u_{k}^{p}}{2 c}\right) \frac{u_{k}^{n+1}-u_{k}^{n}}{\Delta t} \\
w \frac{\partial u}{\partial z} \Rightarrow \frac{1}{4}\left[w_{k-1}^{p}\left(\frac{u_{k-1}^{n}-u_{k}^{n}}{\Delta z_{k d}}+\frac{u_{k-1}^{n+1}-u_{k}^{n+1}}{\Delta z_{k d}}\right)+w_{k}^{p}\left(\frac{u_{k}^{n}-u_{k+1}^{n}}{\Delta z_{k u}}+\frac{u_{k}^{n+1}-u_{k+1}^{n+1}}{\Delta z_{k u}}\right)\right] \\
-\frac{1}{\rho} \frac{\partial p}{\partial x} \Rightarrow\left(1-\frac{\tilde{u}_{\infty}^{n+1}+\tilde{u}_{\infty}^{n}}{2 c}\right) \frac{\tilde{u}_{\infty}^{n+1}-\tilde{u}_{\infty}^{n}}{\Delta t} \\
\frac{\partial}{\partial z}\left(\left(v+v_{t}\right) \frac{\partial u}{\partial z}\right) \Rightarrow \frac{1}{\Delta z_{k}}\left(\left(v+v_{t, k-1}^{n}\right) \frac{u_{k-1}^{n+1}-u_{k}^{n+1}}{\Delta z_{k d}}-\left(v+v_{t, k}^{n}\right) \frac{u_{k}^{n+1}-u_{k+1}^{n+1}}{\Delta z_{k u}}\right)
\end{gathered}
$$

(with $n$ the time step number and $p$ the prediction for the next time step). Hereby, $\tilde{u}_{\infty}$ is the free stream horizontal orbital velocity component, known in advance for every time step. So in the free model formulation, the pressure term is also known in advance. This is not the case in the match model formulation (discretization not elaborated here). The discretizations in both step 1 and 2 result in a tri-diagonal matrix, which is solved using Gaussian elimination.

The solution method above could result in a time-step dependent numerical contribution to the wave-averaged current, caused by slight phase shifts between the various components of discretized terms. However, this error can never be larger than the numerical error from the discretization of the horizontal advective term without the predictor:

$$
\left.u \frac{\partial u}{\partial x}\right|_{k} \Rightarrow-\left.\frac{u}{c} \frac{\partial u}{\partial t}\right|_{k} \Rightarrow-\frac{u_{k}^{n}}{c} \frac{u_{k}^{n+1}-u_{k}^{n}}{\Delta t}
$$

An analytical estimation of this error for a sinusoidal wave gives:

$$
\int_{t=0}^{T}\left(-\frac{u^{n}}{c} \frac{\partial u^{n+1 / 2}}{\partial t}\right) d t=\int_{t=0}^{T}\left\{-\hat{u}^{2} k \sin (\omega t-k x) \cos \left(\omega t-k x+\frac{\omega \Delta t}{2}\right)\right\} d t=T \frac{\hat{u}^{2} k}{2} \sin \left(\frac{\omega \Delta t}{2}\right)
$$


This indicates that (for reasonable values of $\Delta t$ ) the maximum error depends linearly on the time step. A time step criteria can be determined from equation (C.10) and the requirement that the numerical contribution should be at least two orders smaller than the progressive wave streaming estimated with the analytical expression of Longuet-Higgins [1958]. This would yield:

$$
\frac{\pi^{2} \Delta t}{T} \leq 0.01
$$

Representative model settings are (for simulations with the BL2-free model version): a time step of $1 / 1000$ times the wave period, a simulation length of 100 waves and a grid of 150 layers exponentially divided over 2.5 times the estimated boundary layer thickness, leading to simulation durations of around 3 minutes that allow for systematic exploration of the parameter domain.

As a check, it may be noted that the BL2-free model version perfectly reproduces the analytical solution of [Longuet-Higgins, 1958] for streaming under progressive sinusoidal waves when run with constant viscosity, and the numerical results of Holmedal and Myrhaug [2009, fig 7] when run with $k-\varepsilon$ closure. The theoretical model validation on the analytical solutions is shown in Figure C.2.

(a)

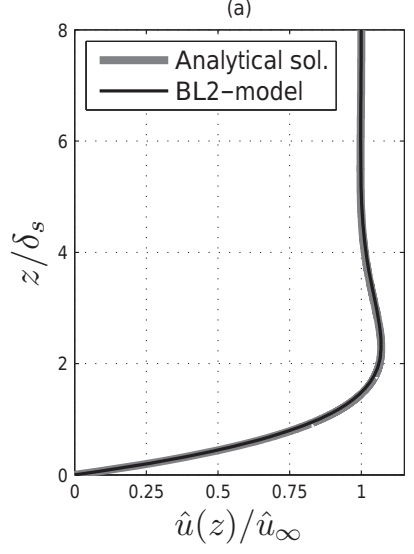

(b)

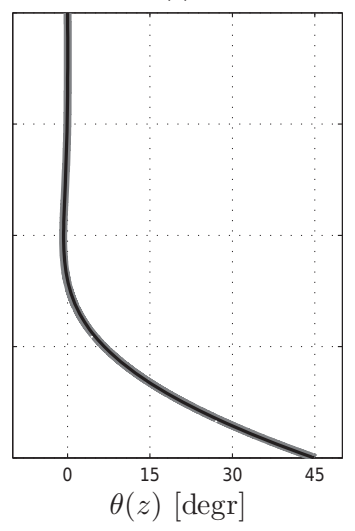

(c)

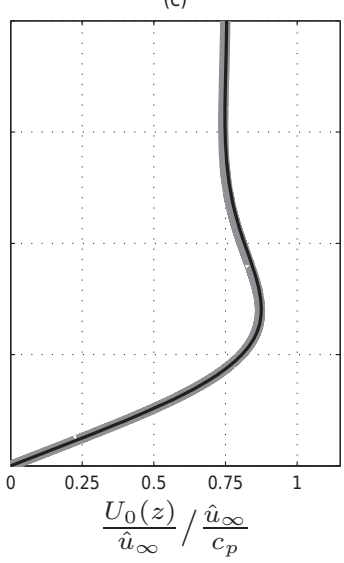

Figure C.2: Theoretical model validation with analytically obtained (normalized) vertical profiles of (a) the amplitude and (b) the phase of the horizontal component of the orbital velocity, and (c) the period-averaged current. (Compare Chapter 1, Figure 1.2, and see appendix A for the mathematical expressions). 


\section{APPENDIX D: SHAPE EXPRESSION}

(First referred to in section 2.4.1).

Equation (2.19) has been derived from the momentum balance (2.1) with the following steps:

1) Averaging over the wave period:

$$
\frac{\partial \overline{u w}}{\partial z}=-\frac{1}{\rho} \frac{\partial \bar{p}}{\partial x}+\frac{\partial}{\partial z}\left\{\overline{\left(v+v_{t}\right) \frac{\partial u}{\partial z}}\right\}
$$

2) Integration over $z$ :

$$
\overline{u w}=-\frac{1}{\rho} \frac{\partial \bar{p}}{\partial x} z+C_{1}+\overline{\left(v+v_{t}\right) \frac{\partial u}{\partial z}}
$$

3) Choice of integration constants such that all typical boundary layer terms are zero outside the boundary layer; no shear stress at the upper boundary $(z=h)$ :

$$
\left(\overline{u w}-\overline{u w}_{\infty}\right)=-\frac{1}{\rho} \frac{\partial \bar{p}}{\partial x}(z-h)+\overline{\left(v+v_{t}\right) \frac{\partial u}{\partial z}}
$$

4) Decomposition of turbulent viscosity and velocity into a period-averaged (overbar) and waverelated (tilde) part and rearrangement of terms to express the mean current as a result of all other contributions:

$$
\left(v+\bar{v}_{t}\right) \frac{\partial \bar{u}}{\partial z}=\left(\overline{u w}-\overline{u w}_{\infty}\right)+\frac{1}{\rho} \frac{\partial \bar{p}}{\partial x}(z-h)-\overline{\tilde{v}_{t} \frac{\partial \tilde{u}}{\partial z}}
$$

with $\infty$ denoting the edge of the boundary layer. 


\section{APPENDIX E: HORIZONTAL SEDIMENT ADVECTION AND ADAPTATION TIME SCALE $T_{\mathrm{A}}$}

(Belonging to section 3.4.2/3.5.1).

\section{Analytical illustration of the effect of horizontal sediment advection}

The contribution of intra-wave gradients in horizontal advection to sediment transport in the direction of wave propagation can be analytically illustrated as follows: moving with the wave propagation speed $c_{\mathrm{p}}$, the material derivative of a steady harmonic oscillation is zero for all quantities (equation (4.2)), including the sediment flux $f=\tilde{u} c$ :

$$
\frac{\partial f}{\partial t}+c_{p} \frac{\partial f}{\partial x}=0
$$

We substitute this equality into the sediment balance, neglecting all vertical sediment exchange:

$$
\frac{\partial c}{\partial t}+\frac{\partial \tilde{u} c}{\partial x}=\frac{\partial}{\partial t}\left\{c-\frac{\tilde{u} c}{c_{p}}\right\}=0
$$

By integration, an expression for $c$ can be derived showing the variation of $c$ with $\tilde{u}$. Taylor expansion around $\tilde{u} / c_{\mathrm{p}} \approx 0$ yields an approximation valid for $\tilde{u} / c_{\mathrm{p}}<<1$ ( $\alpha$ is the integration constant):

$$
c(x, t)=\alpha\left(1-\frac{\tilde{u}(x, t)}{c_{p}}\right)^{-1} \approx \alpha\left(1+\frac{\tilde{u}(x, t)}{c_{p}}\right)
$$

Multiplication with $\tilde{u}$ gives an expression for the flux $f$ that shows the onshore contribution to period averaged sediment transport:

$$
f(x, t) \approx \alpha \tilde{u}+\frac{\alpha \tilde{u}^{2}}{c_{p}} \rightarrow \bar{f} \approx \frac{\alpha \hat{u}^{2}}{2 c_{p}}
$$

\section{Derivation of $T_{\mathrm{a}}$, time scale of adaptation}

The proposed time scale $T_{\mathrm{a}}$ to include the effects of horizontal gradients in sediment advection has been derived from the advection-diffusion equation for sediment:

$$
c_{t}+(u c)_{x}+(w c)_{z}=\left(w_{s} c+\varepsilon c_{z}\right)_{z}
$$

Here subscripts $t, x$, and $z$ denote derivatives. The vertical sediment flux $\varphi$ being:

$$
\varphi=-\left\{\left(w_{s}-w\right) c+\varepsilon c_{z}\right\}
$$

the sediment balance can be written as: 


$$
c_{t}+(u c)_{x}=-\varphi_{z}
$$

We integrate this expression from the bottom $z=0$ to a constant level in the wave boundary layer $z=\delta$ where the sediment concentration (and vertical flux) become negligible (for example the maximum stirring height or 10 times the sheet-flow layer thickness). Subsequently, we shift integration and differentiation and divide all terms by thickness $\delta$ to obtain:

$$
C_{t}+(\overline{u c})_{x}=\frac{\varphi(0)}{\delta}
$$

where capital and over bar denote depth-averaging. $u$ and $c$ are not uniformly distributed over the vertical. Using a distribution coefficient $\alpha$, we express the second term in the free stream velocity $u_{\infty}$ and the depth-averaged concentration $C$, such that $\overline{u c}=\alpha u_{\infty} C$. With this approach, we follow the approximation of Galappatti and Vreugdenhil [1985] for shallow gradually varying flows. Next, we split up the second term in equation (E.8) in two separate derivatives:

$$
\left(\alpha u_{\infty} C\right)_{x}=\alpha u_{\infty}(C)_{x}+\alpha C\left(u_{\infty}\right)_{x}
$$

The first is subsequently rewritten using the transformation $\frac{\partial}{\partial x}=-\frac{1}{c_{p}} \frac{\partial}{\partial t}$ for uniform waves (with $c_{\mathrm{p}}$ the propagation speed of the wave). The second term is rewritten assuming a constant ratio $\xi$ between free stream velocity $u_{\infty}$ and depth-averaged velocity $U$ and using flow continuity over the transport layer:

$$
\frac{\partial u_{\infty}}{\partial x}=\frac{\partial \xi U}{\partial x}=\xi \frac{\partial U}{\partial x}=-\xi \frac{w(\delta)}{\delta}
$$

As a result, we can write equation (E.8) as:

$$
\left\{1-\frac{\alpha u_{\infty}}{c_{p}}\right\} C_{t}-\alpha \xi C \frac{w(\delta)}{\delta}=\frac{\varphi(0)}{\delta}
$$

The vertical sediment flux $\varphi$ at the bottom is the net result of pick-up and deposition: $\varphi(0)=p_{u p}-d e p$, with $p_{\text {up }}$ a function of the instantaneous bed-shear stress and dep the result of vertical settling of sand near the bed. With the near bed concentration $c_{\text {bed }}$ related to the depthaveraged concentration $C$ through a shape coefficient $\gamma>>1, \operatorname{dep}=w_{s} c_{\text {bed }}=w_{s} \gamma C$. Substitution into equation (E.11) gives: 


$$
\left\{1-\frac{\alpha u_{\infty}}{c_{p}}\right\} C_{t}=\frac{1}{\delta}\left\{p_{u p}-w_{s} \gamma C+\alpha \xi C w(\delta)\right\}
$$

The vertical orbital velocities in the wave boundary layer are generally smaller or of the same order of magnitude as the sand settling velocity, i.e. $w(\delta) \leq w_{\mathrm{s}}$. Next $\alpha$ and $\xi$ are of order $\mathrm{O}(1)$, so that $\gamma>>(\alpha \xi)$. Therefore, the third term on the right-hand side of equation (E.12) can be neglected in comparison with the second term. This results in the relaxation expression:

$$
C_{t}=\frac{\frac{\gamma w_{s}}{\delta}\left\{\frac{p_{u p}}{\gamma w_{s}}-C\right\}}{\left\{1-\frac{\alpha u_{\infty}}{c_{p}}\right\}} ; \quad \text { or } \quad C_{t}=\frac{\gamma\left(C_{e q}-C\right)}{T_{a}}
$$

with:

$$
C_{e q}=\frac{p_{u p}}{\gamma w_{s}} ; \quad \text { and } \quad T_{a}=\frac{\delta}{w_{s}}\left\{1-\frac{\alpha u_{\infty}}{c_{p}}\right\}
$$




\section{APPENDIX F: DRAG-RELATED TERMS IN MOMENTUM AND ENERGY EQUATIONS}

(First referred to in section 4.2.2).

\section{Momentum:}

The drag related fluid-particle interaction terms in the momentum equations are obtained by finding the ensemble averaged of the drag force. With Favre averaging, the ensemble averaging is applied over the momentum per unit mass of each phase. This results in the following expressions/definitions for the corresponding mean $\tilde{u}$ and fluctuation $\Delta u$ of the fluid and sediment velocity $u^{f}$ and $u^{s}$ :

$$
\tilde{u}^{f}=\frac{\overline{(1-\phi) u^{f}}}{(1-\bar{\phi})} ; \quad \overline{(1-\phi) \Delta u^{f}}=0 ; \quad \tilde{u}^{s}=\frac{\overline{\phi u^{s}}}{\bar{\phi}} ; \quad \overline{\phi \Delta u^{s}}=0
$$

Using decomposition $u=\tilde{u}+\Delta u$, the drag force can be expressed as:

$$
\bar{F}_{D}=\overline{\beta \phi\left(u^{f}-u^{s}\right)}=\beta\left(\bar{\phi} \tilde{u}^{f}+\overline{\phi \Delta u^{f}}-\bar{\phi} \tilde{u}^{s}-\overline{\phi \Delta u^{s}}\right)
$$

Using equation (F.1), part 4, the last term of equation (F.2) can be omitted, leaving:

$$
\bar{F}_{D}=\beta \bar{\phi}\left(\tilde{u}^{f}-\tilde{u}^{s}\right)+\beta \overline{\phi \Delta u^{f}}
$$

The first RHS term is the drag force due to the mean velocity difference between water and sediment. The second RHS term is closed using a gradient transport and - for the horizontal gradient - subsequently transformed to the time derivative:

$$
\beta \overline{\phi \Delta u^{f}}=-\beta \frac{v_{f t}}{\sigma_{c}} \frac{\partial \bar{\phi}}{\partial x}=\frac{\beta}{c} \frac{v_{f t}}{\sigma_{c}} \frac{\partial \bar{\phi}}{\partial t}
$$

with $c$ the wave propagation speed, $v_{\mathrm{ft}}$ the turbulent viscosity and $\sigma_{\mathrm{c}}$ the Prandt-Schmidt number.

\section{Energy:}

The $k$-equation describes the rate of change of the ensemble averaged kinetic energy of the turbulent fluctuations $(k)$. The turbulent kinetic energy is the total ensemble averaged kinetic energy $(K)$ minus the kinetic energy of the ensemble averaged flow $(\widetilde{K})$. The procedure to obtain the $k$ equation is as follows:

1) multiply fluid momentum equation with $u$ to get kinetic energy 

2) apply decomposition
3) apply ensemble averaging to determine the $K$
4) multiply ensemble averaged fluid momentum equation with $\tilde{u}$ to get $\widetilde{K}$
5) apply $k=K-\widetilde{K}$
6) do this for all directions and sum up the energy of the fluctuations

The drag related terms in the $k$-equation are obtained by applying this procedure on the drag term in the momentum equation. The contribution of drag (in x-direction) to $K$ is:

$$
\begin{aligned}
& D R A G_{K}=-\beta \overline{\phi u^{f}\left(u^{f}-u^{s}\right)}=-\beta\left[\overline{\phi u^{f} u^{f}}-\overline{\phi u^{f} u^{s}}\right] \\
& =-\beta\left[\overline{\phi\left(\tilde{u}^{f}+\Delta u^{f}\right)\left(\tilde{u}^{f}+\Delta u^{f}\right)}-\overline{\phi\left(\tilde{u}^{f}+\Delta u^{f}\right)\left(\tilde{u}^{s}+\Delta u^{s}\right)}\right] \\
& =-\beta\left[\bar{\phi} \tilde{u}^{f} \tilde{u}^{f}+\overline{\phi \Delta u^{f}} 2 \tilde{u}^{f}+\overline{\phi \Delta u^{f} \Delta u^{f}}-\bar{\phi} \tilde{u}^{f} \tilde{u}^{s}-\overline{\phi \Delta u^{f} u^{f}}-\overline{\phi \Delta u^{f}} \tilde{u}^{s}-\overline{\phi \Delta u^{f} \Delta u^{s}}\right]
\end{aligned}
$$

The contribution of drag to $\widetilde{K}$ is:

$$
D R A G_{\widetilde{K}}=-\beta \bar{\phi} \tilde{u}^{f}\left(\tilde{u}^{f}-\tilde{u}^{s}\right)-\beta \tilde{u}^{f} \overline{\phi \Delta u^{f}}=-\beta\left[\bar{\phi} \tilde{u}^{f} \tilde{u}^{f}-\bar{\phi}^{\tilde{u}^{f}} \tilde{u}^{s}+\tilde{u}^{f} \overline{\phi \Delta u^{f}}\right]
$$

Subtracting equation (F.6) from (F.5) yields the drag contribution to the $k$-equation:

$$
\begin{aligned}
& D R A G_{k}=D R A G_{K}-D R A G_{\widetilde{K}} \\
& =-\beta\left[\overline{\phi \Delta u^{f}} \tilde{u}^{f}+\overline{\phi \Delta u^{f} \Delta u^{f}}-\overline{\phi \Delta u^{f}} \tilde{u}^{s}-\overline{\phi \Delta u^{f} \Delta u^{s}}\right] \\
& =-\beta\left[\overline{\phi \Delta u^{f}}\left(\tilde{u}^{f}-\tilde{u}^{s}\right)+\overline{\phi \Delta u^{f}\left(\Delta u^{f}-\Delta u^{s}\right)}\right]
\end{aligned}
$$

The first term is again modeled with the gradient transport assumption. Considering $x$ and $z$ direction, this yields:

$$
\beta \frac{v_{f t}}{\sigma_{c}} \frac{\partial \bar{\phi}}{\partial x}\left(\tilde{u}^{f}-\tilde{u}^{s}\right)+\beta \frac{v_{f t}}{\sigma_{c}} \frac{\partial \bar{\phi}}{\partial z}\left(\widetilde{w}^{f}-\tilde{w}^{s}\right)
$$

In Hsu et al. [2004], we find for the sum of these terms:

$$
\beta \frac{v_{f t}}{\sigma_{c}} \frac{\partial \bar{\phi}}{\partial z}\left(\widetilde{w}^{f}-\widetilde{w}^{s}\right)
$$


In that study the horizontal gradient can be left out following the original model assumption of horizontally uniform flow. Another argument, also valid in horizontally non-uniform flow, is that $x$ term is small compared to the $z$-term because the vertical velocity difference is higher under influence of gravity and the concentration gradient is higher because the boundary layer is small compared to the wave length. In Yu et al. [2010], we finally find:

$$
\beta \frac{v_{f t}}{\sigma_{c}} \frac{\partial \bar{\phi}}{\partial z}\left(\widetilde{w}^{f}-\widetilde{w}^{s}\right) \quad \rightarrow \quad\left(\rho_{s}-\rho_{f}\right) g \frac{v_{f t}}{\sigma_{c}} \frac{\partial \bar{\phi}}{\partial z}
$$

which actually assumes an equilibrium between drag force from settling and (reduced) gravity force.

The second term of equation (F.7), last row, is modeled using the parameter $\alpha$ characterizing the degree the particles fluctuations follow the fluid fluctuations:

$$
-\beta \overline{\phi \Delta u^{f}\left(\Delta u^{f}-\Delta u^{s}\right)}=-\beta(1-\alpha) \overline{\phi \Delta u^{f} \Delta u^{f}}
$$

The fluctuation correlation for all directions together is related to $k$ itself. With the approximation:

$$
\overline{\phi \Delta u^{f} \Delta u^{f}} \approx 2 \bar{\phi} k_{f}
$$

the contribution can be expressed as:

$$
-\beta(1-\alpha) \overline{\phi \Delta u^{f} \Delta u^{f}}=-2 \beta(1-\alpha) \bar{\phi} k
$$

applied in the $k$-equation. 


\section{APPENDIX G: PARTICLE MOTION AND STOKES NUMBER RANGE}

(First referred to in section 4.4.2).

This appendix discusses the motion of a particle in accelerating fluid. We discuss an approximation of the transfer function between particle and fluid motion, determined by the relative density and the Stokes number. Finally we discuss the Stokes number range relevant for this study.

We consider a volume of fluid that accelerates under influence of a pressure gradient and the acceleration of a particle within that volume under influence of the pressure gradient and the drag force. The momentum equations read:

$$
\begin{gathered}
\rho_{f} \frac{d u_{f}}{d t}=-\frac{d P}{d x} \\
\rho_{s} \phi \frac{d u_{s}}{d t}=-\phi \frac{d P}{d x}+\phi \beta\left(u_{f}-u_{s}\right)
\end{gathered}
$$

Herein, the volume concentration $\phi$ is small (so no feedback on the flow is present), the fluid motion is assumed uniform and the sediment is described as a continuum (to allow for comparison with section 4.2.2).

Substitution of (G.1) into (G.2) yields:

$$
\frac{d u_{s}}{d t}+a u_{s}=b \frac{d u_{f}}{d t}+a u_{f} ; \quad \text { with } \quad a=\frac{\beta}{\rho_{s}}=\frac{1}{T_{p}} ; \quad b=\frac{\rho_{f}}{\rho_{s}} ;
$$

Considering fluid and sediment motion as a summation of Fourier components with angular frequency $\omega$, we define for each component:

$$
u_{f}=\hat{u}_{f} e^{i \omega t} ; \quad u_{s}=\eta e^{i \varphi} \hat{u}_{f} e^{i \omega t} ;
$$

with $\eta$ the amplitude ratio and $\varphi$ a phase difference. Substitution into equation (G.3) gives:

$$
\eta e^{i \varphi}=\frac{i \omega b+a}{i \omega+a}
$$

and $\eta$ and $\varphi$ are respectively:

$$
\eta=\sqrt{\operatorname{Re}^{2}(A)+\operatorname{Im}^{2}(A)} ; \quad \beta=\operatorname{atan}\{\operatorname{Im}(A) / \operatorname{Re}(A)\}
$$

with $A$ the RHS of equation (G.5). For coefficients $a$ and $b$ as in (G.3), and with fluid time scale $T_{f}=$ $2 * \pi / \omega$, the transfer function can be written as: 


$$
\eta e^{i \varphi}=\frac{i \omega b+a}{i \omega+a}=\frac{2 \pi \frac{T_{p}}{T_{f}} \frac{\rho_{f}}{\rho_{s}}-i}{2 \pi \frac{T_{p}}{T_{f}}-i}
$$

This shows that the transfer function is a function of the relative time scale and relative density ratio only. When we would consider only viscous drag (first part of $\beta$-expression, equation (4.6)), ratio $T_{p}$ / $T_{f}$ could be expressed as:

$$
\frac{T_{p}}{T_{f}}=\frac{\rho_{s}}{\beta} \frac{1}{T_{f}}=\frac{1}{2 \pi} \frac{1}{18} \frac{\rho_{s}}{\rho_{f}} \frac{d^{2} \omega}{v}
$$

with two independent parameters $\rho_{\mathrm{s}} / \rho_{\mathrm{f}}$ and $d^{2} \omega / v$, the latter being the Stokes number (note that often also $T_{\mathrm{p}} / T_{\mathrm{f}}$ itself is called the Stokes number).

(a)

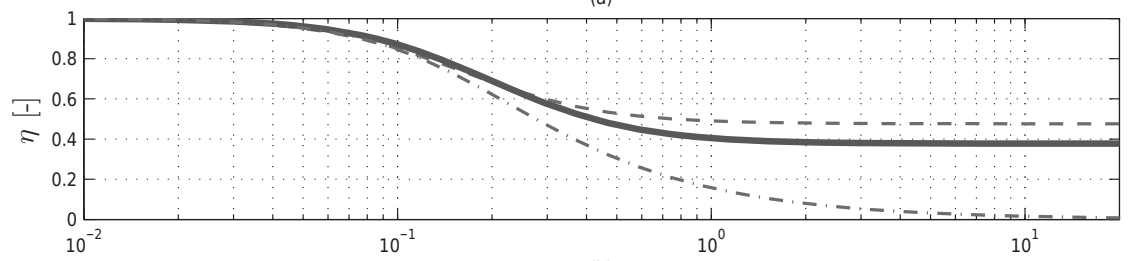

(b)

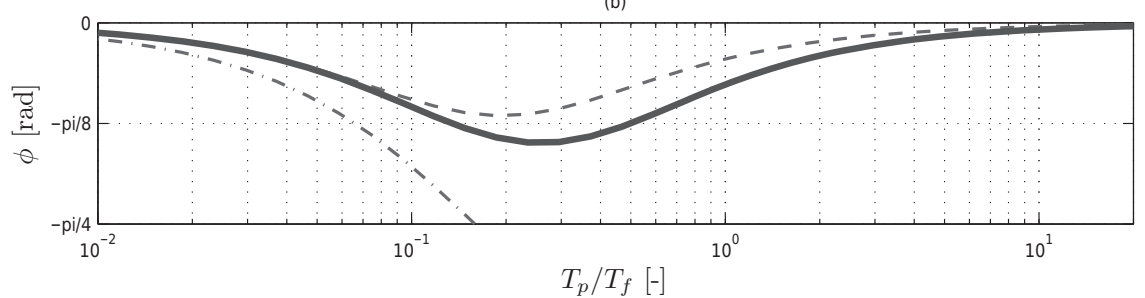

(c)

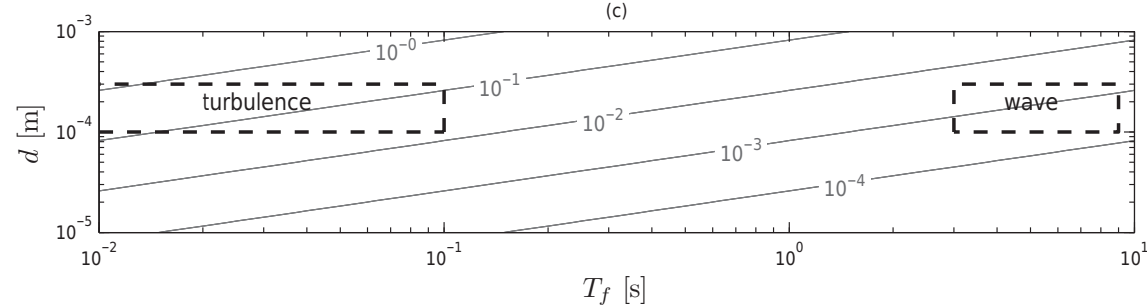

Figure G.1: (a) Amplitude ratio $\eta$ and (b) phase difference $\varphi$ between fluid and particle motion as function of time scale ratio $T_{\mathrm{p}} / T_{\mathrm{f}}$ for density ratio $\rho_{\mathrm{s}} / \rho_{\mathrm{f}}=2.65$. (c) $T_{\mathrm{p}} / T_{\mathrm{f}}$ as function of $T_{\mathrm{f}}$ and $d$. Combination of information from (c) and (a) shows that the fine and medium sized sands $(0.1<d<0.3 \mathrm{~mm})$ may be expected to follow very well the (first) harmonic of the wave, while fluid sediment differences will appear for the turbulent motion (say $T_{\mathrm{f}}<0.1 \mathrm{~s}$ ). Line: transfer function of equation (G.7); Dashed line: particle motion due to pressure gradient, drag force and added mass; Dash-dotted line: particle motion due to drag force only. 
Amplitude ratio and phase difference are shown in Figure G.1 (a) and (b) and indications of relevant parameter values are given in panel (c). We conclude that fluid-grain velocity differences may be expected for the turbulent motion and thus that drag will become relevant for the turbulence model.

This appendix is based on Hjelmfelt and Mockros [1966] and Hinze [1975]. Hjelmfelt and Mockros [1966] also investigated the effect of excluding / including other terms in the momentum balance of the sediment (include added mass effect, exclude pressure gradient for accelerating fluid). This results in alternative values for coefficients $a$ and $b$, while equation (G.5) stays unchanged. Two alternatives are added to Figure G.1. We learn that the effect of added mass is limited, and that the $\alpha$ functions applied in the two phase model resemble the transfer function from drag force only.

\section{Addendum to Table 3.2}

Run ID-codes of the UVP measurements of Schretlen [2012] used for analysis and simulations in Chapter 3;

\begin{tabular}{llll}
\hline & Condition & $\boldsymbol{n}$ : number of UVP runs & UVP run ID-codes \\
\hline & & 5 & $206 \mathrm{a}, 258 \mathrm{a}, 258 \mathrm{~b}, 259 \mathrm{~b}, 259 \mathrm{c}$, \\
1 & $1265 \mathrm{~m}$ & 7 & $195,238 \mathrm{a}, 238 \mathrm{~b}, 246 \mathrm{a}, 246 \mathrm{~b}, 254 \mathrm{~b}, 255 \mathrm{~b}$ \\
2 & $1550 \mathrm{~m}$ & 4 & $154,154 \mathrm{a}, 225 \mathrm{a}, 243 \mathrm{~b}$ \\
3 & $1565 \mathrm{~m}$ & 4 & $169 \mathrm{a}, 176 \mathrm{a}, 239 \mathrm{a}, 255 \mathrm{~b}$ \\
4 & $1575 \mathrm{~m}$ & 3 & $135,140,144$ \\
\hline 5 & $1065 \mathrm{f}$ & 7 & $104 \mathrm{a}, 104 \mathrm{~b}, 110,110 \mathrm{a}, 124,132,170$ \\
6 & $1265 \mathrm{f}$ & 4 & $067,071,075,084$ \\
7 & $1550 \mathrm{f}$ & 5 & $044,047,056,174 \mathrm{a}, 174 \mathrm{~b}$ \\
8 & $1565 \mathrm{f}$ & 2 & 089,173 \\
9 & $1575 \mathrm{f}$ & & \\
& & &
\end{tabular}




\section{REFERENCES}

Abreu, T., P. a. Silva, F. Sancho, and A. Temperville (2010), Analytical approximate wave form for asymmetric waves, Coastal Engineering, 57(7), 656-667, doi:10.1016/j.coastaleng.2010.02.005.

Ahilan, R. V., and J. F. A. Sleath (1987), Sediment transport in oscillatory flow over flat beds, Journal of Hydraulic Engineering, 113(3), 308-322.

Al-Salem, A. A. (1993), Sediment transport in oscillatory boundary layers under sheet-flow conditions, PhD Thesis, Delft University of Technology, Delft, The Netherlands.

Amoudry, L., T.-J. Hsu, and P. L.-F. Liu (2008), Two-phase model for sand transport in sheet flow regime, Journal of Geophysical Research, 113(C3), 1-15, doi:10.1029/2007JC004179.

Amoudry, L. O. (2012), Assessing sediment stress closures in two-phase sheet flow models, Advances in Water Resources, doi:10.1016/j.advwatres.2012.03.011.

Asano, T. (1990), Two-phase flow model on oscillatory sheet flow, in Proceedings of 22th International Conference on Coastal Engineering, pp. 2372-2384, ASCE, New York, U.S.

Bagnold, R. a. (1954), Experiments on a Gravity-Free Dispersion of Large Solid Spheres in a Newtonian Fluid under Shear, Proceedings of the Royal Society A: Mathematical, Physical and Engineering Sciences, 225(1160), 49-63, doi:10.1098/rspa.1954.0186.

Bailard, J. A. (1981), An energetics total load sediment transport model for a plane sloping beach, Journal of Geophysical Research, 86(C11), 10938-10954.

Baptist, M. J., V. Babovic, J. R. Uthurburu, M. Keijzer, R. E. Uittenbogaard, A. Mynett, and A. Verwey (2007), On inducing equations for vegetation resistance, Journal of Hydraulic Research, 45(4), 435-450.

Blondeaux, P., G. Vittori, A. Bruschi, F. Lalli, and V. Pesarino (2012), Steady streaming and sediment transport at the bottom of sea waves, Journal of Fluid Mechanics, 697, 115-149, doi:10.1017/jfm.2012.50.

Borsje, B. W., P. C. Roos, W. M. Kranenburg, and S. J. M. H. Hulscher (2011), Modeling sandwave formation in a numerical shallow water model, in 7th IAHR symposium on River, Coastal and Estuarine Morphodynamics, edited by X. Shao, Z. Wang, and G. Wang, Beijing, China.

Borsje, B. W., W. M. Kranenburg, P. C. Roos, J. Matthieu, and S. J. M. H. Hulscher (n.d.), Modeling sand wave formation in a numerical shallow water model: the role of suspended sediment,

Bosboom, J., and G. Klopman (2000), Intra-wave sediment transport modeling, in Proceedings of 27th International Conference on Coastal Engineering, edited by B. L. Edge, pp. 2453-2466, Sydney, Australia, 2000.

Breugem, A. (2012), Transport of suspended particles in turbulent open channel flows, PhD-thesis, Delft University of Technology, The Netherlands.

Campbell, L. J., T. O’Donoghue, and J. S. Ribberink (2007), Wave boundary layer velocities in oscillatory sheet flow, in Proceedings of 30th International Conference on Coastal Engineering, edited by J. McKee Smith, pp. 2207-2219, World Scientific Publishing, San Diego, California, USA, 2006.

Carstensen, S., B. M. Sumer, and J. Fredsøe (2010), Coherent structures in wave boundary layers. Part 
1. Oscillatory motion, Journal of Fluid Mechanics, 646, 169, doi:10.1017/S0022112009992825.

Cavallaro, L., P. Scandura, and E. Foti (2011), Turbulence-induced steady streaming in an oscillating boundary layer: On the reliability of turbulence closure models, Coastal Engineering, 58(4), 290304, doi:10.1016/j.coastaleng.2010.10.001.

Chen, C. P., and P. E. Wood (1985), Chen-C, Canadian Journal of Chemical Engineering, 63(3), Pages 349-360.

Chen, X., Y. Li, X. Niu, D. Chen, and X. Yu (2011), A two-phase approach to wave-induced sediment transport under sheet flow conditions, Coastal Engineering, 58(11), 1072-1088, doi:10.1016/j.coastaleng.2011.06.003.

Conley, D. C., S. Falchetti, I. P. Lohmann, and M. Brocchini (2008), The effects of flow stratification by non-cohesive sediment on transport in high-energy wave-driven flows, Journal of Fluid Mechanics, 610, 43-67, doi:10.1017/S0022112008002565.

Danon, H., M. Wolfshtein, and G. Hetsroni (1977), Numerical calculations of two-phase turbulent round jet, Int. J. Multiphase Flow, 3(1960), 223-234.

Davies, A. G. (1980), Field observations of the threshold of sand motion in a transitional wave boundary layer, Coastal Engineering, 4, 23-46.

Davies, A. G., and Z. Li (1997), Modelling sediment transport beneath regular symmetrical and asymmetrical waves above a plane bed, Continental Shelf Research, 17(5), 555-582, doi:10.1016/S0278-4343(96)00048-9.

Davies, A. G., and C. Villaret (1999), Eulerian drift induced by progressive waves above rippled and very rough beds, Journal of Geophysical Research, 104(C1), 1465-1488, doi:10.1029/1998JC900016.

Davies, A. G., L. C. Van Rijn, J. S. Damgaard, J. Van de Graaff, and J. S. Ribberink (2002), Intercomparison of research and practical sand transport models, Coastal Engineering, 46, 1-23.

Dibajnia, M., and A. Watanabe (1992), Dibaijnia_Watanabe_1992, in Proceedings 23rd International Conference on Coastal Engineering, pp. 2015-2028, ASCE, Venice.

Dibajnia, M., and A. Watanabe (1998), Transport rate under irregular sheet flow conditions, Coastal Engineering, 35(3), 167-183, doi:10.1016/S0378-3839(98)00034-9.

Dohmen-Janssen, C. M. (1999), Grain size influence on sediment transport in oscillatory sheet-flow, phase lags and mobile-bed effects, PhD Thesis, Delft University of Technology, Delft, The Netherlands.

Dohmen-Janssen, C. M., and D. M. Hanes (2002), Sheet flow dynamics under monochromatic nonbreaking waves, Journal of Geophysical Research, 107(C10), 1-21, doi:10.1029/2001JC001045.

Dohmen-Janssen, C. M., D. F. Kroekenstoel, W. N. Hassan, and J. S. Ribberink (2002), Phase lags in oscillatory sheet flow: experiments and bed load modelling, Coastal Engineering, 46(1), 61-87, doi:10.1016/S0378-3839(02)00056-X.

Dong, P., and K. Zhang (1999), Two-phase flow modelling of sediment motions in oscillatory sheet flow, Coastal Engineering, 36(2), 87-109, doi:10.1016/S0378-3839(98)00052-0.

Drew, D. A. (1976), Production and dissipation of energy in the turbulent flow of a particle-fluid mixture, with some results on drag reduction, , 543-547. 
Elfrink, B., D. M. Hanes, and B. G. Ruessink (2006), Parameterization and simulation of near bed orbital velocities under irregular waves in shallow water, Coastal Engineering, 53(11), 915-927, doi:10.1016/j.coastaleng.2006.06.002.

Elghobashi, S. E., and T. W. Abou-Arab (1983), A two-equation turbulence model for two-phase flows, Physics of Fluids, 26(4), 931, doi:10.1063/1.864243.

Fredsøe, J., A. O.H., and S. Silberg (1985), Distribution of suspended sediment in large waves, Journal of Waterway, Port, Coastal and Ocean Engineering, 111(6), 1041-1059.

Fredsøe, J., and R. Deigaard (1992), Mechanics of coastal sediment transport, World Scientific, Advanced Series on Ocean Engineering Vol.3.

Fredsøe, J., B. M. Sumer, A. Kozakiewicz, L. H. C. Chua, and R. Deigaard (2003), Effect of externally generated turbulence on wave boundary layer, Coastal Engineering, 49, 155 - 183, doi:10.1016/S0378-3839(03)00032-2.

Fuhrman, D. R., J. Fredsøe, and B. M. Sumer (2009a), Bed slope effects on turbulent wave boundary layers: 1. Model validation and quantification of rough-turbulent results, Journal of Geophysical Research, 114(C3), 1-16, doi:10.1029/2008JC005045.

Fuhrman, D. R., J. Fredsøe, and B. M. Sumer (2009b), Bed slope effects on turbulent wave boundary layers: 2. Comparison with skewness, asymmetry, and other effects, Journal of Geophysical Research, 114(C3), 1-19, doi:10.1029/2008JC005053.

Galappatti, G., and C. B. Vreugdenhil (1985), A depth-integrated model for suspended sediment transport, Journal of Hydrualic Research, 23(4), 359-377.

Gallagher, E. L., S. Elgar, and R. T. Guza (1998), Observations of sand bar evolution on a natural beach, Journal of Geophysical Research, 103(C2), 3203-3215.

Gonzalez Rodriquez, D. (2009), Wave boundary layer hydrodynamics and cross-shore sediment transport in the surf zone, PhD-thesis Massachusetts Insitute of Technology, Cambridge, MA, USA.

Gonzalez Rodriquez, D., and O. S. Madsen (2011), Prediction of net bedload transport rates obtained in oscillating water tunnels and applicability to real surf zone waves, in Proceedings of 32nd International Conference on Coastal Engineering, edited by J. McKee Smith and P. Lynett, pp. 111, CERC, Shanghai, China, 2010.

Guizien, K. (2003), 1DV bottom boundary layer modeling under combined wave and current: Turbulent separation and phase lag effects, Journal of Geophysical Research, 108(C1), 1-15, doi:10.1029/2001JC001292.

Hagatun, K., and K. J. Eidsvik (1986), Oscillating turbulent boundqary layer with suspended sediments, Journal of Geophysical Research, 91(C11), 13045-13055.

Hanes, D. M., and D. L. Inman (1985), Observations of rapidly flowing granular-fluid materials, Journal of Fluid Mechanics, 150, 357-380, doi:10.1017/S0022112085000167.

Hassan, W. N. M. (2003), Transport of size-graded and uniform sediment under oscillatory sheet-flow conditions, PhD Thesis, University of Twente, Enschede, The Netherlands.

Hassan, W. N., and J. S. Ribberink (2005), Transport processes of uniform and mixed sands in oscillatory sheet flow, Coastal Engineering, 52(9), 745-770, doi:10.1016/j.coastaleng.2005.06.002. 
Hassan, W. N. M., and J. S. Ribberink (2010), Modelling of sand transport under wave-generated sheet flows with a RANS diffusion model, Coastal Engineering, 57(1), 19-29, doi:10.1016/j.coastaleng.2009.08.009.

Henderson, S. M., J. S. Allen, and P. A. Newberger (2004), Nearshore sandbar migration predicted by an eddy-diffusive boundary layer model, Journal of Geophysical Research, 109(C6), 1-15, doi:10.1029/2003JC002137.

Henriquez, M., A. J. H. M. Reniers, B. G. Ruessink, and M. J. F. Stive (2012), Vortex tubes in the wave bottom boundary layer, in NCK-days 2012: Jubilee Conference Proceedings 20th NCK-Days, edited by W. M. Kranenburg, E. M. Horstman, and K. M. Wijnberg, pp. 143-146, University of Twente, Enschede, the Netherlands.

Hinze, J. O. (1975), Turbulence, 2nd ed., edited by B. J. Clark, McGraw-Hill series in mechanical engineering.

Hjelmfelt, A. T., and L. F. Mockros (1966), Motion of discrete particles in a turbulent fluid, Appl. Sci. Res., 16, 149-161.

Hoefel, F., and S. Elgar (2003), Wave-induced sediment transport and sandbar migration, Science (New York, N.Y.), 299(5614), 1885-7, doi:10.1126/science.1081448.

Holmedal, L. E., D. Myrhaug, and H. Rue (2003), The sea bed boundary layer under random waves plus current, Continental Shelf Research, 23(7), 717-750, doi:10.1016/S0278-4343(03)00020-7.

Holmedal, L. E., D. Myrhaug, and K. J. Eidsvik (2004), Sediment suspension under sheet flow conditions beneath random waves plus current, Continental Shelf Research, 24(17), 2065-2091, doi:10.1016/j.csr.2004.06.021.

Holmedal, L. E., and D. Myrhaug (2006), Boundary layer flow and net sediment transport beneath asymmetrical waves, Continental Shelf Research, 26(2), 252-268, doi:10.1016/j.csr.2005.11.004.

Holmedal, L. E., and D. Myrhaug (2009), Wave-induced steady streaming, mass transport and net sediment transport in rough turbulent ocean bottom boundary layers, Continental Shelf Research, 29(7), 911-926, doi:10.1016/j.csr.2009.01.012.

Hsu, T.-J., J. T. Jenkins, and P. L.-F. Liu (2003), On two-phase sediment transport: Dilute flow, Journal of Geophysical Research, 108(C3), doi:10.1029/2001JC001276.

Hsu, T.-J., J. T. Jenkins, and P. L.-F. Liu (2004), On two-phase sediment transport: sheet flow of massive particles, Proceedings of the Royal Society A: Mathematical, Physical and Engineering Sciences, 460(2048), 2223-2250, doi:10.1098/rspa.2003.1273.

Hsu, T.-J., and D. M. Hanes (2004), Effects of wave shape on sheet flow sediment transport, Journal of Geophysical Research, 109(C5), 1-15, doi:10.1029/2003JC002075.

Hsu, T.-J., S. Elgar, and R. T. Guza (2006), Wave-induced sediment transport and onshore sandbar migration, Coastal Engineering, 53(10), 817-824, doi:10.1016/j.coastaleng.2006.04.003.

Hulscher, S. J. M. H. (1996), Tidal-induced large-scale regular bed form patterns in a three-dimensional shallow water model, Journal of Geophysical Research, 101(C9), 20727-20744.

Jackson, P. S. (1981), On the displacement height in the logarithmic velocity profile, Journal of Fluid Mechanics, 111, 15-25.

Jenkins, J. T., and D. M. Hanes (1998), Collisional sheet flows of sediment driven by a turbulent fluid, Fluid Mechanics, 370, 29-52. 
Johns, B. (1970), On the mass transport induced by oscillatory flow in a turbulent boundary layer, Journal of Fluid Mechanics, 43(01), 177-185, doi:10.1017/S0022112070002306.

Johns, B. (1977), Residual flow and boundary shear stress in the turbulent bottom layer beneath waves, Journal of Physical Oceanography, 7, 733-738.

Jonsson, I. G. (1966), Wave boundary layers and friction factors, in Proceedings of 10th conference on Coastal Engineering, vol. II, pp. 127-148, ASCE, Tokyo, Japan, 1966.

Jonsson, I. G. (1980), A new approach to oscillatory rough turbulent boundary layers, Ocean Engineering, 7, 109-152.

Justesen, P. (1988), Turbulent wave boundary layers, Thesis Techn. Univ. of Denmark, Inst. of Hydrodynamics and Hydraulic Engineering ISVA, Denmark.

Kamphuis, J. W. (1975), Friction factor under oscillatory waves, Journal of the waterways harbors and coastal engineering division, 101(2), 135-144.

Klopman, G. (1994), Vertical structure of the flow due to waves and current, Progress report, H840.30, Part II, Delft, The Netherlands.

Kranenburg, W. M., J. S. Ribberink, J. L. M. Schretlen, and R. E. Uittenbogaard (2013), Sand transport beneath waves: the role of progressive wave streaming and other free surface effects, Journal of Geophysical Research, doi:10.1029/2012JF002427

Kranenburg, W. M., J. S. Ribberink, R. E. Uittenbogaard, and S. J. M. H. Hulscher (2012), Net currents in the wave bottom boundary layer: on wave shape streaming and progressive wave streaming, Journal of Geophysical Research, 117(F03005), doi:10.1029/2011JF002070.

Launder, B. E., and D. B. Spalding (1972), Mathematical models of turbulence, Academic Press, London.

Lescinski, J., and H. T. Özkan-Haller (2004), Modeling bar migration using predicted velocity time series, in Proceedings of 29th International Conference on Coastal Engineering, pp. 2729-2741, Lisbon, Portugal, 2004.

Lesser, G. R., J. A. Roelvink, J. A. T. M. van Kester, and G. S. Stelling (2004), Development and validation of a three-dimensional morphological model, Coastal Engineering, 51(8-9), 883-915, doi:10.1016/j.coastaleng.2004.07.014.

Li, M., S. Pan, and B. A. O'Connor (2008), A two-phase numerical model for sediment transport prediction under oscillatory sheet flows, Coastal Engineering, 55(12), 1159-1173, doi:10.1016/j.coastaleng.2008.05.003.

Li, Z., and A. G. Davies (1996), Towards prediction sediment transport in combined wave-current flow, Journal of waterway, port, coastal and ocean engineering, 122(4), 157-164.

Long, W., J. T. Kirby, and T.-J. Hsu (2006), Cross shore sandbar migration predicted by a tima domain boussinesq model incorportation undertow, in Proceedings of 30th International Conference on Coastal EngineeringCoastal Engineering, pp. 2655-2667, San Diego, California, USA, 2006.

Longuet-Higgins, M. S. (1953), Mass transport in water waves, Philosophical Transactions of the Royal Society of London. Series A, Mathematical and Physical Sciences, 245(903), 535-581.

Longuet-Higgins, M. S. (1958), The mechanics of the boundary-layer near the bottom in a progressive wave, in Proceedings of 6th conference on Coastal Engineering, pp. 184-193, ASCE, Berkely, USA, 1958. 
Madsen, O. S., and W. D. Grant (1976), Quantitative description of sediment transport by waves, in Proceedings of 15th International Conference on Coastal Engineering, pp. 1093-1112, ASCE, Honolulu, Hawai, USA.

McCave, I. N. (1971), Sand waves in the North Sea off the coast of Holland, Marine Geology, 10, 199225.

McTigue, D. F. (1981), Mixture theory for suspended sediment transport, Journal of the Hydraulics Division of ASCE, 107(6), 659-673.

Nielsen, P. (1992), Coastal bottom boundary layers and sediment transport, World Scientific, Advanced Series on Ocean Engineering Vol.4.

Nielsen, P., K. V. D. Wal, and L. Gillan (2002), Vertical fluxes of sediment in oscillatory sheet flow, Coastal Engineering, 45, 61-68.

Nielsen, P. (2006), Sheet flow sediment transport under waves with acceleration skewness and boundary layer streaming, Coastal Engineering, 53(9), 749-758, doi:10.1016/j.coastaleng.2006.03.006.

O'Donoghue, T., and S. Wright (2004a), Flow tunnel measurements of velocities and sand flux in oscillatory sheet flow for well-sorted and graded sands, Coastal Engineering, 51(11-12), 11631184, doi:10.1016/j.coastaleng.2004.08.001.

O'Donoghue, T., and S. Wright (2004b), Concentrations in oscillatory sheet flow for well sorted and graded sands, Coastal Engineering, 50(3), 117-138, doi:10.1016/j.coastaleng.2003.09.004.

Ozdemir, C. E., T.-J. Hsu, and S. Balachandar (2010), A numerical investigation of fine particle laden flow in an oscillatory channel: the role of particle-induced density stratification, Journal of Fluid Mechanics, 665(2010), 1-45, doi:10.1017/S0022112010003769.

Reniers, a. J. H. M., E. B. Thornton, T. P. Stanton, and J. a. Roelvink (2004), Vertical flow structure during Sandy Duck: observations and modeling, Coastal Engineering, 51(3), 237-260, doi:10.1016/j.coastaleng.2004.02.001

Ribberink, J. S. (1998), Bed-load transport for steady flows and unsteady oscillatory flows, Coastal Engineering, 34, 59-82.

Ribberink, J. S., and A. A. Al-Salem (1994), Sediment transport in oscillatory boundary layers in cases of rippled beds and sheet flow, Journal of Geophysical Research, 99(C6), 12707-12727.

Ribberink, J. S., and A. A. Al-Salem (1995), Sheet flow and suspension of sand in oscillatory boundary layers, Coastal Engineering, 25(3-4), 205-225, doi:10.1016/0378-3839(95)00003-T.

Ribberink, J. S., and Z. Chen (1993), Sediment transport of fine sand under asymmetric oscillatory flow, Report, Delft, The Netherlands.

Ribberink, J. S., J. J. van der Werf, T. O'Donoghue, and W. N. M. Hassan (2008), Sand motion induced by oscillatory flows: Sheet flow and vortex ripples, Journal of Turbulence, 9(20), 1-32, doi:10.1080/14685240802220009.

Richardson, J. F., and W. N. Zaki (1954), Sedimentation and fluidisation: Part I, Transactions of the Institution of Chemical Engineers, 32, S82-S100, doi:10.1016/S0263-8762(97)80006-8.

Rienecker, M. M., and J. D. Fenton (1981), A Fourier approximation method for steady water waves, Journal of Fluid Mechanics, 104, 119-137.

Rodi, W. (1984), Turbulence models and their applications in hydraulics. A state of the art review, 2nd 
ed., IAHR, Delft.

Ruessink, B. G., G. Ramaekers, and L. C. van Rijn (2012), On the parameterization of the free-stream non-linear wave orbital motion in nearshore morphodynamic models, Coastal Engineering, 65, 56-63, doi:10.1016/j.coastaleng.2012.03.006.

Ruessink, B. G., T. J. J. van den Berg, and L. C. van Rijn (2009), Modeling sediment transport beneath skewed asymmetric waves above a plane bed, Journal of Geophysical Research, 114(C11), 1-14, doi:10.1029/2009JC005416.

Ruessink, B. G., Y. Kuriyama, A. J. H. M. Reniers, J. A. Roelvink, and D. J. R. Walstra (2007), Modeling cross-shore sandbar behavior on the timescale of weeks, Journal of Geophysical Research, 112(F3), 3010, doi:10.1029/2006JF000730.

Rundqvist, R., C. Ljus, and B. van Wachem (2005), Experimental and numerical investigation of particle transport in a horizontal pipe, AIChE Journal, 51(12), 3101-3108, doi:10.1002/aic.10571.

Schretlen, J. L. M. (2012), Sand transport under full-scale progressive surface waves, $\mathrm{PhD}$-thesis, University of Twente, Enschede, The Netherlands (http://doc.utwente.nl/80884).

Schretlen, J. L. M., J. S. Ribberink, and T. O’Donoghue (2011), Boundary layer flow and sand transport, in Proceedings of 32nd International Conference on Coastal Engineering, edited by J. McKee Smith and P. Lynett, pp. 1-14, CERC, Shanghai, China, 2010.

Scott, N. V., T.-jian Hsu, and D. Cox (2009), Steep wave, turbulence, and sediment concentration statistics beneath a breaking wave field and their implications for sediment transport, Continental Shelf Research, 29(20), 2303-2317, doi:10.1016/j.csr.2009.09.008.

Sleath, J. F. A. (1987), Turbulent oscillatory flow over rough beds, Journal of Fluid Mechanics, 182, 369, doi:10.1017/S0022112087002374.

Soulsby, R. L. (1997), Dynamics of Marine Sands, Thomas Telford, London.

Squires, K. D., and J. K. Eaton (1994), Effect of selective modification of turbulence on two-equation models for particle-laden turbulent flows, Journal of Fluids Engineering, 116(4), 778.

Sumer, B. M., A. Kozakiewicz, J. Fredsoe, and R. Deigaard (1996), Velocity and concentration profiles in sheet-flow layer of movable bed, Journal of Hydraulic Engineering, (October), 549-558.

Svendsen, I. A. (1984), Mass flux and undertow in a surf zone, Coastal Engineering, 8, 347-365.

Svendsen, I. A. (2006), Introduction to nearshore hydrodynamics, World Scientific Publishing, Advanced Series on Ocean Engineering Vol.24.

Swart, D. H. (1974), Offshore sediment transport and equilibrium beach profiles, Delft Hydraulics Laboratory Publication no. 131, Delft, The Netherlands.

Teakle, I. A. L. (2006), Coastal boundary layer and sediment transport modelling, PhD-thesis, University of Queensland, Australia, 2006.

Trowbridge, J., and D. Young (1989), Sand transport by unbroken water waves under sheet flow conditions, Journal of Geophysical Research, 94(C8), 10971-10991.

Trowbridge, J., and O. S. Madsen (1984a), Turbulent wave boundary layers 1. Model formulation and first-order solution, Journal of Geophysical Research, 89(C5), 7989-7997.

Trowbridge, J., and O. S. Madsen (1984b), Turbulent wave boundary layers 2. Second-order theory and mass transport, Journal of Geophysical Research, 89(C5), 7999-8007. 
Uittenbogaard, R. E. (2000), 1DV Simulation of wave current interaction, in Proceedings of 27th International Conference on Coastal Engineering, vol. 2, edited by B. L. Edge, pp. 255-268, ASCE, Sydney, Australia, 2000.

Uittenbogaard, R. E., J. Bosboom, and T. Van Kessel (2001), Numerical simulation of wave-current driven sand transport: Theoretical background of the beta-release of the POINT-SAND model, Report, Z2899.10, Delft, The Netherlands.

Uittenbogaard, R., and G. Klopman (2001), Numerical simulation of wave-current driven sediment transport, in Proceedings of Coastal Dynamics 2001, vol. 3, pp. 1-11, Lund, Sweden, 2001.

Van der A, D. A. (2010), Effects of acceleration skewness on oscillatory boundary layers and sheet flow sand transport, PhD-thesis University of Aberdeen, UK.

Van der A, D. A., J. S. Ribberink, J. J. van der Werf, and T. O'Donoghue (2011), New practical model for sand transport induced by non-breaking waves and currents, in Proceedings of 32nd International Conference on Coastal Engineering, edited by J. McKee Smith and P. Lynett, p. 14, CERC, Shanghai, China, 2010.

Van der A, D. A., T. O’Donoghue, A. G. Davies, and J. S. Ribberink (2011), Experimental study of the turbulent boundary layer in acceleration-skewed oscillatory flow, Journal of Fluid Mechanics, 684, 251-283, doi:10.1017/jfm.2011.300.

Van der A, D. a., T. O’Donoghue, and J. S. Ribberink (2010), Measurements of sheet flow transport in acceleration-skewed oscillatory flow and comparison with practical formulations, Coastal Engineering, 57(3), 331-342, doi:10.1016/j.coastaleng.2009.11.006.

Van der Werf, J. J. (2006), Sand transport over rippled beds in oscillatory flow, PhD Thesis, University of Twente, Enschede, The Netherlands.

Van der Werf, J. J., J. S. Doucette, T. O'Donoghue, and J. S. Ribberink (2007), Detailed measurements of velocities and suspended sand concentrations over full-scale ripples in regular oscillatory flow, Journal of Geophysical Research, 112(F2).

Van der Werf, J. J., V. Magar, J. Malarkey, K. Guizien, and T. O’Donoghue (2008), 2DV modelling of sediment transport processes over full-scale ripples in regular asymmetric oscillatory flow, Continental Shelf Research, 28(8), 1040-1056, doi:10.1016/j.csr.2008.02.007.

Van der Werf, J., H. Nomden, J. Ribberink, D.-J. Walstra, and W. Kranenburg (2012), Application of a new sand transport formula within the cross-shore morphodynamic model Unibest-TC, in Proceedings of 33nd International Conference on Coastal Engineering, Santander, Spain, 2012.

Van Doorn, T. (1981), Experimental investigation of near-bottom velocities in water waves with and without a current, Report, M1423, Part I, Delft, The Netherlands.

Van Rijn, L. C. (1990), Principles of fluid flow and surface waves in rivers, estuaries, seas and oceans, Aqua Publications, Amsterdam, The Netherlands.

Van Rijn, L. C. (1993), Principles of sediment transport in rivers, estuaries and coastal seas, Aqua Publications, Amsterdam, The Netherlands.

Van Rijn, L. C. (2007), Unified view of sediment transport by currents and waves. I: Initiation of motion, bed roughness, and bed-load transport, Journal of Hydraulic Engineering, 133(6), 649, doi:10.1061/(ASCE)0733-9429(2007)133:6(649).

Van Rijn, L. C., P. K. Tonnon, and D. J. R. Walstra (2011), Numerical modelling of erosion and accretion of plane sloping beaches at different scales, Coastal Engineering, 58(7), 637-655, 
doi:10.1016/j.coastaleng.2011.01.009.

Vincent, C. E., and D. M. Hanes (2002), The accumulation and decay of near-bed suspended sand concentration due to waves and wave groups, Continental Shelf Research, 22(14), 1987-2000, doi:10.1016/S0278-4343(02)00051-1.

Wenneker, I., A. van Dongeren, J. Lescinski, D. Roelvink, and M. Borsboom (2011), A Boussinesqtype wave driver for a morphodynamical model to predict short-term morphology, Coastal Engineering, 58(1), 66-84, doi:10.1016/j.coastaleng.2010.08.007.

Wilcox, D. C. (1994), Turbulence modeling in CFD, 1st ed., Ind., Inc., La Canada, Calif.

Wilson, K. C. (1989), Friction of wave-induced sheet flow, Coastal Engineering, 12, 371-379.

Winterwerp, J. C. (2001), Stratification effects by cohesive and noncohesive sediment, Journal of Geophysical Research, 106(C10), 22559-22574.

Wright, S. (2002), Well-sorted and graded sand is oscillatory sheet-flow, PhD-thesis University of Aberdeen, UK, Aberdeen.

Yu, X., T.-J. Hsu, and D. M. Hanes (2010), Sediment transport under wave groups: Relative importance between nonlinear waveshape and nonlinear boundary layer streaming, Journal of Geophysical Research, 115(C2), 1-18, doi:10.1029/2009JC005348.

Yu, X., T.-J. Hsu, J. T. Jenkins, and P. L.-F. Liu (2012), Predictions of vertical sediment flux in oscillatory flows using a two-phase, sheet-flow model, Advances in Water Resources, 48, 2-17, doi:10.1016/j.advwatres.2012.05.012.

Zhang, C., J. Zheng, Y. Wang, and Z. Demirbilek (2011), Modeling wave-current bottom boundary layers beneath shoaling and breaking waves, Geo-Marine Letters, 31(3), 189-201, doi:10.1007/s00367-010-0224-9.

Zhang, Y., and C. S. Campbell (1992), The interface between fluid-like and solid-like behaviour in twodimensional granular flows, Journal of Fluid Mechanics, 237, 541-568, doi:10.1017/S0022112092003525.

Zyserman, J. A., and J. Fredsoe (1994), Data analysis of bed concentration of suspended sediment, Journal of Hydraulic Engineering, 120(9), 1021-1042. 


\section{LIST OF FIGURES}

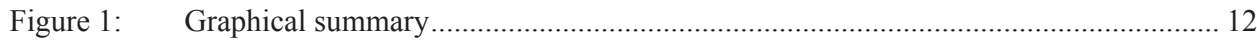

Figure 1.1: Orbital motions under waves for various water depths ............................................. 16

Figure 1.2: Vertical profiles of $\hat{u}, \theta$ and $U_{0}$ : analytical solutions for constant viscosity ............... 17

Figure 1.3: Schematic representation of the generation of progressive wave streaming ............... 18

Figure 1.4: Schematic representation of the generation of wave shape streaming. ....................... 18

Figure 1.5: Parameter space delineation for sheet-flow beneath non-breaking waves. .................. 20

Figure 1.6: Laboratory facilities for research on wave-induced sediment transport ...................... 22

Figure 1.7: Schematic overview of thesis methodology and outline.............................................. 28

Figure 2.1: Delineation of flow regimes in $A / k_{\mathrm{N}}-R e$ space, with position of validation cases..... 37

Figure 2.2: $\quad$ Model-data comparison on $U_{0}, \hat{u}$ and $\theta$ for validation case 1 .................................... 41

Figure 2.3: Model-data comparison on $U_{0}, \hat{u}$ and $\theta$ for validation case 2, 3 and 4 ..................... 43

Figure 2.4: Model-model-data comparison on $U_{0}$; results from BL2, BL2-sinus \& BL1, analytical results of Trowbridge and Madsen [1984b] and data of Van Doorn [1981] .............. 44

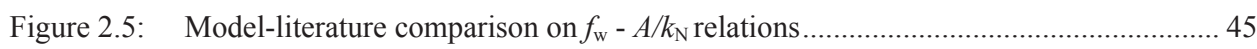

Figure 2.6: Contributions to the mean shear stress for validation case 1 to 4 ............................... 47

Figure 2.7: Non-dimensional streaming velocities as function of $k h$ and $a / h$ for $A / k_{\mathrm{N}}=100 \ldots \ldots . .49$

Figure 2.8: Streaming velocities from full model simulations, simulations with wave shape effect only, and simulations with free surface effect only

Figure 2.9: Streaming velocities as function of $k h$ for various values of $A / k_{\mathrm{N}}$, compared with analytical results of Trowbridge and Madsen [1984b] ............................................ 51

Figure 2.10: Contributions to the mean shear stress for changing pressure gradients .................... 53

Figure 2.11: Net sand transport rates $<q_{\mathrm{s}}>$ for case MI and MH of Dohmen-Janssen and Hanes [2002], determined from $\tau_{\mathrm{b}}(t)$ both without and with progressive wave streaming .... 55

Figure 2.12: Third order velocity moment $\left\langle u^{3}\right\rangle$ as function of $z$, for simulations without and with

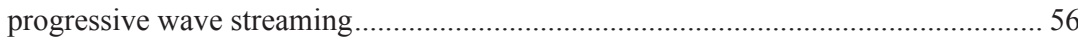

Figure 3.1: Model-data comparison on $U_{0}, \hat{u}$ and $\theta$ for condition $1065 \mathrm{f}$ of Schretlen [2012] ...... 67

Figure 3.2: Model-model-data comparison on $U_{0}$; comparing BL1-SED, BL2-HYDRO \& BL2SED simulations with experimental data of Schretlen [2012] .................................... 68

Figure 3.3: Model-data comparison on $U_{0}$ for multiple wave and bed conditions.......................... 69

Figure 3.4: Model-data comparison on $\left\langle q_{\mathrm{s}}>\right.$ under regular, velocity-skewed waves ..................... 72

Figure 3.5: Model-data comparison on trends in $\left\langle q_{\mathrm{s}}\right\rangle$ as function of $\left\langle u_{\mathrm{red}}{ }^{3}\right\rangle$............................... 74

Figure 3.6: Sensitivity analysis considering $U_{0} \&<q_{\mathrm{s}}>$ for changing $\sigma_{\mathrm{t}}, k_{\mathrm{N}} \& p$.......................... 75

Figure 3.7: Quantification of the return current effect by comparing $\left\langle q_{\mathrm{s}}\right\rangle_{\text {open }} \&\left\langle q_{\mathrm{s}}\right\rangle_{\text {closed }} \ldots . . . . . . . . .78$

Figure 3.8: Surplus of horizontal velocity and sediment concentration from the various advective

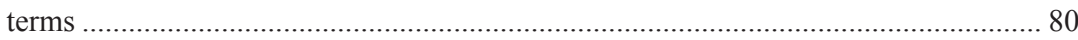

Figure 3.9: $\quad\left\langle q_{\mathrm{s}}>\right.$ against $<\tilde{u}_{\infty}{ }^{3}>$ with the various advective terms switched on/off ...................... 82

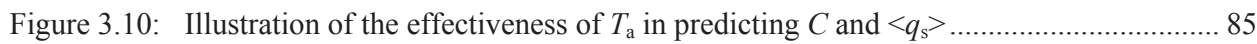

Figure 4.1: Schematization of vertical regions in the two-phase model ...................................... 96

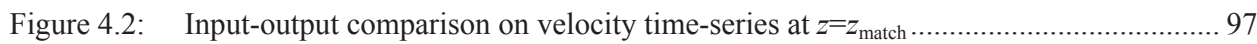


Figure 4.3: Model-model-data comparison on erosion depth $\delta_{\mathrm{e}}$ : data of O'Donoghue and Wright [2004] for various grain sizes and model results obtained with various $\alpha$-functions 100

Figure 4.4: Particle-fluid fluctuation coefficient $\alpha$ and damping proportionality $(1-\alpha) / T_{\mathrm{p}}$ as

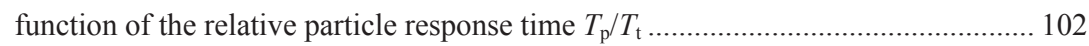

Figure 4.5: Model-data comparison on intra wave concentrations, case MA7515 ...................... 104

Figure 4.6: Model-data comparison on intra wave concentrations, case FA7515 ...................... 104

Figure 4.7: Model-data comparison on intra wave horizontal sediment velocities...................... 106

Figure 4.8: Model-data comparison on intra wave horizontal flow velocities ............................. 106

Figure 4.9: Model-data comparison on $U_{0}$ for tunnel \& flume cases, fine and medium sand ..... 107

Figure 4.10: Sensitivity tests considering $\delta_{\mathrm{e}}, c$ and $u$ for changing coefficients in the grain - carrier

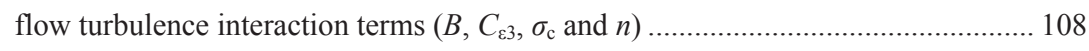

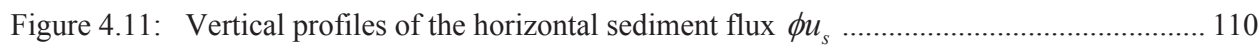

Figure 4.12: Current related and wave relate contribution to the mean flux $\left\langle\phi u_{s}\right\rangle \ldots \ldots \ldots \ldots \ldots \ldots \ldots \ldots . . . . . . .110$

Figure 5.1: Diagram of relation between experiments, intra wave boundary layer modeling, sand transport formulas and morphological modeling .................................................... 120

Figure 5.2: Structure of simple morphodynamic model................................................................ 121

Figure 5.3: Morphology: sandbar migration with and without free surface effects ..................... 123

Figure 5.4: Morphology: mechanisms contribution to sediment fluxes above a sand wave........ 125

Figure 6.1: $\quad\left\langle q_{\mathrm{s}}>\right.$ against $<\tilde{u}_{\infty}{ }^{3}>$ with the various free surface effects switched on/off................. 129

Figure B.1: Histogram of the occurrence of Hs and Tm02 for RWS buoy YM6 ..........................136

Figure C.1: Numerical grid ...................................................................................................137

Figure C.2: $\quad$ Model validation on analytically obtained vertical profiles of $\hat{u}, \theta$ and $U_{0} \ldots \ldots \ldots \ldots \ldots . . .139$

Figure G.1: Amplitude ratio and phase difference between fluid and particle motion ...................148 


\section{LIST OF SYMBOLS}

\section{Abbreviations}

\begin{tabular}{ll}
\hline Abbreviation & Description \\
\hline ADV & Acoustic Doppler Velocity meter \\
AOFT & Aberdeen Oscillatory Flow Tunnel \\
BL & Boundary Layer \\
CCM & Conductivity Concentration Meter \\
DNS & Direct Numerical Simulation \\
EMF & Electromagnetic Flow Meter \\
FLU & Simulation with all advective terms switched on (flume) \\
LDA & Laser-Doppler Anemometry \\
LES & Large Eddy Simulation \\
PIV & Particle Image Velocimetry \\
RANS & Reynolds-Averaged Navier-Stokes (equations) \\
REF & Reference simulation, with all advective terms switched off \\
RMS & Root Mean Square (value) \\
RWS & Rijkswaterstaat (Dutch Ministry of Public Works) \\
TKE & Turbulent Kinetic Energy \\
UVP & Ultrasonic Velocity Profiler \\
WBL & Wave Boundary Layer \\
WRS & Wave Reynolds Stress \\
&
\end{tabular}

\section{Operators}

\begin{tabular}{ll}
\hline Operator & Description \\
\hline$\langle X\rangle$ & Wave period average parameter $X$ \\
$\bar{X}$ & Wave period average parameter $X$ \\
$\widetilde{X}$ & Wave-related component of parameter $X$ \\
$\widetilde{X}$ & Ensemble average parameter $X$ \\
$\widehat{X}$ & Amplitude of parameter $X$ \\
$\vec{X}$ & Parameter $X$ in vector form \\
$\dot{X}$ & Time derivative of parameter $X$ \\
\hline
\end{tabular}




\section{Subscripts}

\begin{tabular}{ll}
\hline Subscript & Description \\
\hline$X_{\infty}$ & Parameter $X$ in the free stream / at edge of wave boundary layer \\
$X_{\text {red }}$ & Reduced parameter $X$ : based on wave-related velocity components only \\
$X_{\mathrm{w}}$ & Parameter $X$ for the fluid phase \\
$X_{\mathrm{f}}, X^{\mathrm{f}}$ & Parameter $X$ for the fluid phase \\
$X_{\mathrm{s}}, X^{\S}$ & Parameter $X$ for the sediment phase \\
\end{tabular}

\section{Roman symbols}

\begin{tabular}{|c|c|c|}
\hline Symbol & Description & Unit \\
\hline$A$ & orbital flow excursion & {$[\mathrm{m}]$} \\
\hline$a$ & wave amplitude & {$[\mathrm{m}]$} \\
\hline$B_{\mathrm{k}}$ & $\begin{array}{l}\text { buoyancy flux, conversion of kinetic energy in potential energy with } \\
\text { upward mixing of sediment }\end{array}$ & {$\left[\mathrm{m}^{2} / \mathrm{s}^{3}\right]$} \\
\hline$C$ & sediment (volume) concentration: depth-averaged & {$\left[\mathrm{m}^{3} / \mathrm{m}^{3}\right]$} \\
\hline$c$ & sediment (volume) concentration & {$\left[\mathrm{m}^{3} / \mathrm{m}^{3}\right]$} \\
\hline$c, c_{\mathrm{p}}$ & wave propagation velocity & {$[\mathrm{m} / \mathrm{s}]$} \\
\hline$c_{\mathrm{b}}$ & reference concentration & {$\left[\mathrm{m}^{3} / \mathrm{m}^{3}\right]$} \\
\hline$c_{\text {bed }}$ & near bed concentration & {$\left[\mathrm{m}^{3} / \mathrm{m}^{3}\right]$} \\
\hline$C_{e q}$ & equilibrium concentration (depth-averaged) & {$\left[\mathrm{m}^{3} / \mathrm{m}^{3}\right]$} \\
\hline$c_{\mathrm{s}}$ & maximum volume concentration of packed sand & {$\left[\mathrm{m}^{3} / \mathrm{m}^{3}\right]$} \\
\hline$c_{\mu}, \mathrm{c}_{1 \varepsilon}, c_{2 \varepsilon}, c_{3 \varepsilon}$ & coefficients in turbulence model (in Ch 4 written as: $C_{\mu}, C_{\varepsilon 1}, C_{\varepsilon 2}, C_{\varepsilon 3}$ ) & {$[-]$} \\
\hline$D, d$ & grain diameter & {$[\mathrm{m}]$} \\
\hline$d_{50}$ & median grain diameter & {$[\mathrm{m}]$} \\
\hline$D_{\mathrm{E}}$ & energy dissipation from bed friction (period averaged) & {$[\mathrm{N} / \mathrm{m} / \mathrm{s}]$} \\
\hline$f_{\mathrm{w}}$ & friction factor & {$[-]$} \\
\hline$g$ & gravitational acceleration & {$\left[\mathrm{m} / \mathrm{s}^{2}\right]$} \\
\hline$H$ & wave height & {$[\mathrm{m}]$} \\
\hline$H_{0}$ & deep water wave height & {$[\mathrm{m}]$} \\
\hline$H_{\mathrm{s}}$ & significant wave height & {$[\mathrm{m}]$} \\
\hline$h$ & water depth & {$[\mathrm{m}]$} \\
\hline$k$ & layer or interface number in vertical direction & {$[-]$} \\
\hline$K$ & total ensemble averaged kinetic energy & {$\left[\mathrm{m}^{2} / \mathrm{s}^{2}\right]$} \\
\hline$k$ & turbulent kinetic energy & {$\left[\mathrm{m}^{2} / \mathrm{s}^{2}\right]$} \\
\hline$k$ & wave number & {$[1 / \mathrm{m}]$} \\
\hline$k_{\mathrm{N}}$ & Nikuradse roughness height & {$[\mathrm{m}]$} \\
\hline$K_{\mathrm{sh}}$ & shoaling coefficient & {$[-]$} \\
\hline
\end{tabular}


Brunt-Väisälä frequency

coefficient in the concentration-dependent correction of the drag

numerical index, e.g. denoting the $n$-th harmonic

percentage of the total number of occurrences

time step number

Rouse number $w_{\mathrm{s}}(\kappa u *)^{-1}$

coefficient in concentration-dependent correction of the settling velocity

pressure

$\left[\mathrm{N} / \mathrm{m}^{2}\right]$

turbulence production

sediment pick-up

flux of particle fluctuation energy $\Theta$

sediment transport rate

velocity skewness parameter

wave Reynolds number $\left(\hat{u}_{1} A / v\right)$

particle Reynolds number $\left(U_{\mathrm{r}} d / v\right)$

velocity skewness measure computed from $u_{\text {red }}$

reproduction quality measure

specific density $\left(\rho_{\mathrm{s}} / \rho_{\mathrm{w}}\right)$

velocity skewness parameter

time

wave period

time scale of adaptation of the sediment concentration

mean period of wave energy spectrum

peak period of wave energy spectrum

time scale of the particle response: measure of the time to accelerate a

single particle from rest to the velocity of the surrounding fluid by drag

amplitude of the horizontal component of the orbital velocity

horizontal velocity component

Ursell number $U=H L^{2} / h^{3}$

friction velocity 


$\begin{array}{lll}V(z) & \text { complex amplitude of the 'defect velocity' } v & {[\mathrm{~m} / \mathrm{s}]} \\ w & \text { vertical velocity component } & {[\mathrm{m} / \mathrm{s}]} \\ w_{\mathrm{s}} & \text { sediment fall velocity or grain settling velocity } & {[\mathrm{m} / \mathrm{s}]} \\ w_{\mathrm{s}, 0} & \text { settling velocity of a single particle in clear fluid } & {[\mathrm{m} / \mathrm{s}]} \\ x & \text { coordinate along horizontal axes } & {[\mathrm{m}]} \\ z & \text { coordinate along vertical axes } & {[\mathrm{m}]} \\ Z & \text { domain height } & {[\mathrm{m}]} \\ z_{0} & \text { roughness length scale } & {[\mathrm{m}]} \\ z_{\mathrm{a}} & \text { level where lower b.c. of sediment balance is applied }(\mathrm{Ch} 3) & {[\mathrm{m}]} \\ z_{\mathrm{b}} & \text { bed level } & {[\mathrm{m}]} \\ z_{\mathrm{m}} & \text { matching level, where model output for } u \text { is forced to match a measured } & {[\mathrm{m}]} \\ & \text { velocity signal } & \end{array}$

\section{Greek symbols}

\begin{tabular}{lll}
\hline Symbol & Description & Unit \\
\hline$\alpha$ & distribution coefficient related to shape of concentration \& velocity profile & {$[-]$} \\
$\alpha$ & parameter characterizing the degree particles follow the fluid fluctuation & {$[-]$} \\
$\beta$ & drag parameter $\beta=\rho_{\mathrm{s}} / T_{p}$ & {$\left[\mathrm{~kg} / \mathrm{m}^{3} / \mathrm{s}\right]$} \\
$\beta$ & acceleration-skewness parameter & {$[-]$} \\
$\beta$ & inverse of the Stokes length $\delta_{\mathrm{s}}$ & {$[1 / \mathrm{m}]$} \\
$\gamma$ & coefficient within $T_{\mathrm{a}}$ related to the shape of the concentration profile. & {$[-]$} \\
$\gamma$ & energy dissipation from inelastic collisions & {$\left[\mathrm{N} / \mathrm{m}^{2} / \mathrm{s}\right]$} \\
$\Delta$ & relative immersed density $\left(\rho_{\mathrm{s}}-\rho_{\mathrm{w}}\right) / \rho_{\mathrm{w}}$ & {$[-]$} \\
$\delta$ & particle entrainment height $($ also measure for the transport layer thickness) & {$[\mathrm{m}]$} \\
$\delta^{*}$ & estimate for the turbulent wave boundary layer thickness & {$[\mathrm{m}]$} \\
$\delta_{\mathrm{e}}$ & erosion depth & {$[\mathrm{m}]$} \\
$\delta_{\mathrm{s}}$ & sheet-flow layer thickness & {$[\mathrm{m}]$} \\
$\delta_{\mathrm{s}}$ & Stokes length $(2 \mathrm{v} / \omega)^{1 / 2}$ & {$[\mathrm{~m}]$} \\
$\delta_{\mathrm{s}}$ & boundary layer thickness estimate according to Sleath $[1987]$ & {$[\mathrm{m}]$} \\
$\Delta t$ & time step & {$[\mathrm{s}]$} \\
$\delta_{\mathrm{v}}$ & thickness of viscous sub-layer & {$[\mathrm{m}]$} \\
$\Delta z$ & layer thickness vertical grid & {$[\mathrm{m}]$} \\
$\varepsilon, \varepsilon_{f}$ & dissipation rate of turbulent kinetic energy $k$ & {$\left[\mathrm{~m}^{2} / \mathrm{s}^{3}\right]$} \\
$\varepsilon$ & porosity & {$\left[\mathrm{m}^{3} / \mathrm{m}^{3}\right]$} \\
$\Theta$ & 'granular temperature': energy of the particle velocity fluctuations & {$\left[\mathrm{m}^{2} / \mathrm{s}^{2}\right]$} \\
$\theta$ & Shields number; non-dimensional bed shear stress & {$[-]$} \\
$\theta$ & phase (lead) & {$[\mathrm{rad}],\left[{ }^{\circ}\right]$} \\
\hline & & \\
\hline & &
\end{tabular}




\begin{tabular}{|c|c|c|}
\hline$\theta_{\mathrm{c}}$ & critical Shields parameter for initiation of motion & {$[-]$} \\
\hline$\theta_{\max }$ & maximum Shields parameter during the wave & {$[-]$} \\
\hline$\theta_{\mathrm{n}}$ & phase lead of the $n$-th harmonic component with respect to the free stream & {$[\mathrm{rad}],\left[^{\circ}\right]$} \\
\hline$\kappa$ & Von Karman constant & {$[-]$} \\
\hline$\mu_{\mathrm{s}}$ & shear viscosity & {$[\mathrm{kg} / \mathrm{m} / \mathrm{s}]$} \\
\hline$v_{\mathrm{ft},}, v_{\mathrm{t}}$ & eddy viscosity & {$\left[\mathrm{m}^{2} / \mathrm{s}\right]$} \\
\hline$\xi_{\mathrm{s}}$ & bulk viscosity & {$[\mathrm{kg} / \mathrm{m} / \mathrm{s}]$} \\
\hline$\rho$ & density & {$\left[\mathrm{kg} / \mathrm{m}^{3}\right]$} \\
\hline$\rho_{\mathrm{m}}$ & density of the local water-sediment mixture $\rho_{\mathrm{m}}=\rho_{\mathrm{w}}+\left(\rho_{\mathrm{s}}-\rho_{\mathrm{w}}\right) c$ & {$\left[\mathrm{~kg} / \mathrm{m}^{3}\right]$} \\
\hline$\rho_{\mathrm{w}}$ & density of water & {$\left[\mathrm{kg} / \mathrm{m}^{3}\right]$} \\
\hline$\sigma_{\mathrm{c}}, \sigma_{\mathrm{t}}$ & Prandtl-Schmidt number, ratio turbulent viscosity and sediment diffusivity & {$[-]$} \\
\hline$\sigma_{\mathrm{k}}, \sigma_{\varepsilon}$ & coefficients from turbulence model, to link diffusivity to $v_{\mathrm{t}}$ & {$[-]$} \\
\hline$\sigma_{\mathrm{p}}$ & coefficient (chosen equal to the turbulence Prandtl-Schmidt number $\sigma_{\mathrm{t}}$ ) & {$[-]$} \\
\hline$\tau$ & stress & {$\left[\mathrm{N} / \mathrm{m}^{2}\right]$} \\
\hline$\tau_{\mathrm{b}}$ & bed shear stress & {$\left[\mathrm{N} / \mathrm{m}^{2}\right]$} \\
\hline$\tau_{\mathrm{b}, \mathrm{m}}$ & $\begin{array}{l}\text { maximum bed shear stress (exerted by a sinusoidal oscillatory flow with a } \\
\text { velocity amplitude } \hat{u}_{1 \infty} \text { ) }\end{array}$ & {$\left[\mathrm{N} / \mathrm{m}^{2}\right]$} \\
\hline$\tau_{\mathrm{b}, \mathrm{WRS}}$ & wave Reynolds stress & {$\left[\mathrm{N} / \mathrm{m}^{2}\right]$} \\
\hline$v$ & kinematic viscosity of water & {$\left[\mathrm{m}^{2} / \mathrm{s}\right]$} \\
\hline$\phi$ & volumetric sediment concentration & {$\left[\mathrm{m}^{3} / \mathrm{m}^{3}\right]$} \\
\hline$\Psi$ & mobility number & {$[-]$} \\
\hline$\psi$ & phase of periodic parameter & {$[\mathrm{rad}],\left[^{\circ}\right]$} \\
\hline$\omega$ & vorticity & {$[1 / \mathrm{s}]$} \\
\hline$\omega$ & wave angular frequency & {$[\mathrm{rad} / \mathrm{s}]$} \\
\hline
\end{tabular}

\section{Some important parameter combinations (non-dimensional)}

\begin{tabular}{lll}
\hline Abbreviation & Description & \\
\hline & & \\
$a / h$ & Relative wave amplitude & {$[-]$} \\
$A / k_{\mathrm{N}}$ & Relative bed roughness & {$[-]$} \\
$k h$ & Relative water depth & {$[-]$} \\
$t / T$ & Normalized phase & {$[-]$} \\
$T_{\mathrm{a}} / T$ & Phase-lag parameter & {$[-]$} \\
$\hat{u} / c$ & Order of magnitude of advective terms compared to first order terms & {$[-]$} \\
$U_{0} \mathrm{c} / \hat{u}_{1}{ }^{2}$ & Normalized streaming velocity & {$[-]$} \\
$w_{\mathrm{s}} / u^{*}$ & Suspension parameter & {$[-]$} \\
& & \\
\hline
\end{tabular}




\section{DANKWOORD / ACKNOWLEDGEMENTS}

Dit proefschrift zou niet tot stand zijn gekomen zonder de ondersteuning van begeleiders, collega's, vrienden en familie. Een woord van dank is daarom zeker op zijn plaats.

Allereerst dank aan Jan Ribberink, wiens al jarenlang bestaande betrokkenheid bij sheet-flow onderzoek en eigen werk op het gebied van zandtransportformules mij verzekerde van interesse in de resultaten van mijn bezigheden. Dank voor de discussies, vragen en opmerkingen, in het bijzonder de kritische. Dank ook aan Suzanne Hulscher voor bijdragen aan het proces op beslissende momenten in het project. Vooral de steun die je uitsprak en de ruimte die je gaf toen ik - in afwijking van wat we tot dan toe probeerden - besloot tot implementatie van de golfvoortplantingseffecten in het raamwerk van het grenslaagmodel, is voor mij van groot belang geweest. Dank aan Rob Uittenbogaard, voor de introductie in de code waar dit proefschrift in zo'n belangrijke mate op rust, en voor de intensieve en inspirerende privé-colleges m.n. in de eerste helft van dit project. $\mathrm{Al}$ heb ik maar een gering deel uitgewerkt: bedankt voor de karrenvrachten aan ideeën en suggesties.

Next, I would like to thank Tom Hsu for providing me the two-phase code and for hosting me at the University of Delaware. It was a privilege to be a guest in your group, where so much interesting topics come together and can be discussed on a range of levels of detail with such dedicated students. Thanks for this also to Xiao, Charlie, Nancy and the others.

This PhD project was strongly related to the international research projects SANTOSS and HYDRALAB III \& IV-SANDS \& WISE. I thankfully acknowledge the contribution from this side, by means of data provision, comments on draft-papers and inspiring discussions during the project meetings. The same applies for the meetings of the user-committee. Although we conferred in continuously changing constitution - or perhaps just because of that-every time it was refreshing to be critically interviewed on the progress and the benefits of the project, and to hear what happens outside university's door.

Ook de (oud-)collega's van WEM mogen niet onvermeld blijven in dit dankwoord. Allereerst Jolanthe Schretlen, voor de gelegenheid mee te kijken en te doen bij de experimenten im Großen Wellenkanal in Hannover, en voor het beschikbaar stellen van de data. Bas, dank voor alle discussies, meer en minder inhoudelijk. Geweldig om hiervan (en dan bedoel ik de eerste categorie) zelfs iets terug te mogen vinden in je papers: de samenwerking met jou en Pieter rondom jouw zandgolfmodeleerwerk was voor mij een van de hoogtepunten van de laatste jaren. Dank ook aan Erik en Kathelijne voor de goede samenwerking rond de NCK-jubileum conferentie. En zonder verder alle bijdragen aan namen te koppelen: WEM-collega's, bedankt voor alle gezelligheid, praktische hulp en mentale support. 
Ook de interesse en ondersteuning van de kant van vrienden, familie en andere sociale verbanden heb ik zeer gewaardeerd, evenals de afleiding die door diverse activiteiten werd verschaft. Ik noem hierbij graag Spirit, kring, koor, G8 \& bestuur Visser (plus aanhang), de familie Balk en de gezinnen De Lange en Kranenburg waar ik samen met Ditske deel van mag uitmaken. Willem en Liesbeth, dank voor jullie luisterend oor en jullie voorbede. Pa en ma, dank voor al die ondersteuning, van nul tot nu, in zoveel verschillende vormen en bij zoveel verschillende zaken.

Lieve Ditske, ook jij hartelijk bedankt! Voor je geduld bij het aanhoren van mijn verhalen, voor je geweldige veerkracht in moeilijke tijden, voor al je vrolijke initiatieven, en je opgeruimde karakter, dat zich openbaart bij het kleinste scheurtje in een wolkendek. Bedankt voor de aanmoedigingen er tegenaan te gaan, en voor de dwangmaatregelen om de boel te laten rusten. En voor de bevrijdende verwijzing naar God, die bovenal te danken is.

Hoe rijk zijn uw gedachten, God, hoe eindeloos in aantal, ontelbaar veel, meer dan er zandkorrels zijn.

Ontwaak ik, dan nog ben ik bij $u$. (uit: psalm 139) 


\section{LIST OF PUBLICATIONS}

\section{Journal articles}

Kranenburg, W.M., J.S. Ribberink, J.L.M. Schretlen and R.E. Uittenbogaard; Sand transport beneath waves: the role of progressive wave streaming and other free surface effects, accepted for publication in J. Geophys. Res., DOI:10.1029/2012JF002427 (Chapter 3)

Kranenburg, W.M., J.S. Ribberink, R.E. Uittenbogaard and S.J.M.H. Hulscher (2012); Net currents in the wave bottom boundary layer: On waveshape streaming and progressive wave streaming, J. Geophys. Res., 117, F03005, DOI:10.1029/2011JF002070 (Chapter 2)

Kranenburg, W.M., J.C. Winterwerp, G.J. de Boer, J.M. Cornelisse and M. Zijlema (2011); SWAN-mud: Engineering Model for Mud-Induced Wave Damping, Journal of Hydraulic Engineering, ASCE, Vol. 137, No. 9, DOI:10.1061/(ASCE)HY.1943-7900.0000370

Borsje, B.W., W.M. Kranenburg, P.C. Roos, M. Jordan and S.J.M.H. Hulscher; The role of suspended load transport in the occurrence of tidal sand waves, under review

Borsje, B.W., P.C. Roos, W.M. Kranenburg and S.J.M.H. Hulscher; Modeling tidal sand wave formation in a numerical shallow water model: the role of turbulence formulation, under review

Schretlen, J.L.M., J.S. Ribberink, W.M. Kranenburg and T. O'Donoghue; Boundary layer velocities measured above mobile beds under full scale progressive surface waves, under review

Van der A, D.A., J.S. Ribberink, J.J. Van der Werf, T. O’Donoghue, R.H. Buijsrogge and W.M. Kranenburg; Practical sand transport formula for non-breaking waves and currents, under review

\section{Conference papers}

Kranenburg, W.M., J.S. Ribberink, and R.E. Uittenbogaard (2011); Sand transport by surface waves: can streaming explain the onshore transport? In: J. McKee Smith and P. Lynett (Eds.), Proceedings of 32nd International Conference on Coastal Engineering, pp. 1-14, CERC, Shanghai, China, 2010

Kranenburg, W.M., J.S. Ribberink, and R.E. Uittenbogaard (2011); Net currents in the wave boundary layer: balance of wave shape and free surface effects. In: P.Wang, J.D. Rosati, and T.M. Roberts (Eds.), Proceedings of Coastal Sediments 2011, edited by, pp. 1499-1513, Miami, Florida, USA, 2011 (best student paper)

Van der Werf, J.J, H. Nomden, J.S. Ribberink, D.-J. Walstra and W.M. Kranenburg (2012); Application of a new sand transport formula within the cross-shore morphodynamic model Unibest-TC. In: Proceedings of 33nd International Conference on Coastal Engineering, Santander, Spain, 2012 
Borsje, B.W., P.C. Roos, W.M. Kranenburg and S.J.M.H. Hulscher (2011); Modeling sand wave evolution in a numerical shallow water model. In: X.Shao, Z.Wang and G.Wang (Eds.), Proceedings of $7^{\text {th }}$ IAHR symposium on River, Coastal and Estuarine Morphodynamics (RCEM), Bejing, China, 2011

\section{Conference proceedings (as editor)}

Kranenburg, W.M., Horstman, E.M. and Wijnberg, K.M., Eds. (2012); NCK-days 2012: Crossing borders in coastal research : jubilee conference proceedings. University of Twente, Department of Water Engineering \& Management, Enschede, the Netherlands. ISBN 9789036533423 (http://proceedings.utwente.nl/164)

\section{Master thesis}

Kranenburg, W.M. (2008); Modelling wave damping by fluid mud, derivation of a dispersion equation and an energy dissipation term and implementation into SWAN; M.Sc.-thesis, Delft University of Technology, Delft, The Netherlands 


\section{ABOUT THE AUTHOR}

Wouter Kranenburg was born on June 6, 1981 in Zoetermeer, The Netherlands. He received his pre-university education at the 'Gereformeerde Scholengemeenschap Randstad' in Rotterdam (1993-1999), followed by a one year education program (Basisjaar) at the Evangelische Hogeschool in Amersfoort.

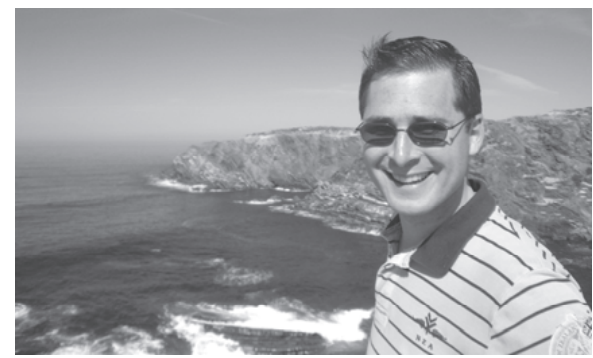

Wouter Kranenburg studied Civil Engineering at Delft University of Technology (2000-2008). He obtained his BSc cum laude in January 2006, after completing his bachelor's with a numerical study on the effects of a bypass in the river Meuse. During his Master's (Hydraulic Engineering, specialization Environmental Fluid Mechanics) he visited the Department of Coastal Engineering \& Port Infrastructure of the Counsel of Scientific and Industrial Research (CSIR) in Stellenbosch, South-Africa, for a research internship concerning long waves generated by wave groups and shoreline accretion by mega nourishment. Wouter obtained his MSc degree cum laude in February 2008. The graduation project, carried out at WL|Delft Hydraulics and the Federal University of Rio de Janeiro (FURJ), Brazil, concerned modeling of wave damping by fluid mud and was awarded with the prof.dr.ir. J.A. Battjes-award for excellent student research on hydraulic engineering.

In June 2008, Wouter joined the Water Engineering and Management group at the University of Twente. He worked on the PhD-research project 'Modeling Sand Transport under Waves in the Sheet-Flow regime', embedded in the international research projects SANTOSS and HYDRALAB IV-SANDS. A part of the project has been carried out at the University of Delaware, USA. The work has been presented in peer reviewed journal articles and contributions to international conferences, one of which was awarded with the 'best student paper award' at Coastal Sediments 2011, Florida, USA.

Next to the activities in the field of numerical modeling - the main focus of the $\mathrm{PhD}$ project $-\mathrm{a}$ wider experience has been developed a.o. by participation in experiments on sand transport under waves in the large scale wave flume in Hannover, Germany, teaching \& supervision of students, and the organization of the NCK-Days 2012, the jubilee conference of the Dutch Center for Coastal Research at the University of Twente. 


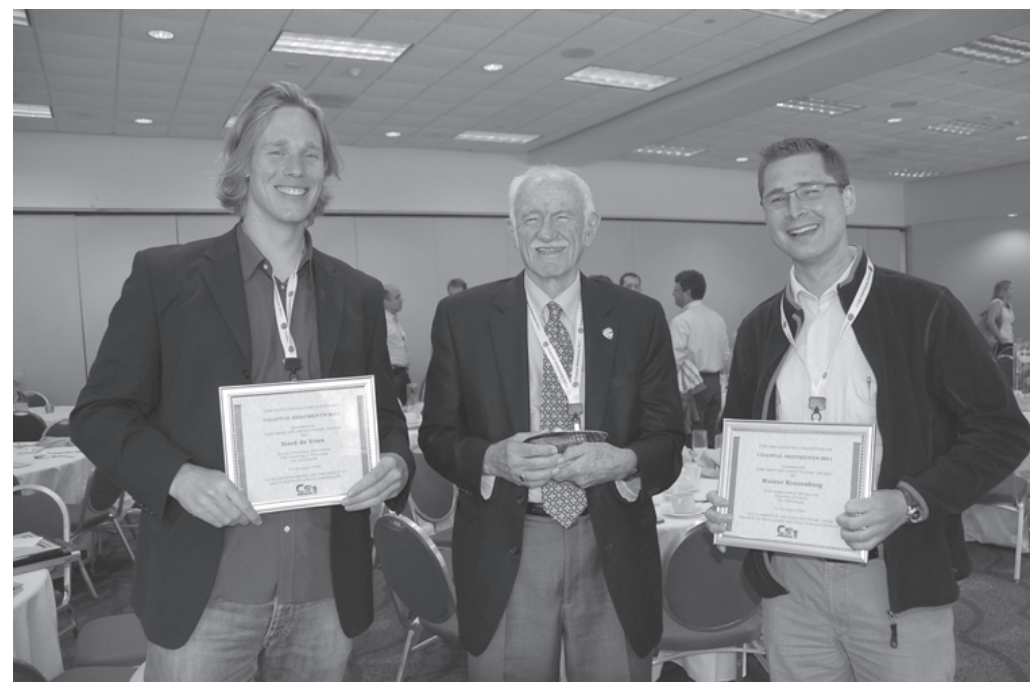

Award ceremony at Coastal Sediment 2011, Miami, Florida, USA. With Coastal Award winner prof. R.G. Dean and fellow student paper award winner Sierd de Vries (left). 
In the near-shore zone, energetic sea waves generate sheet-flow sand transport. The progressive nature of the waves also induces an onshore directed current near the bed.

This thesis describes the development of process-based numerical models of the wave bottom boundary layer and investigates progressive wave effects on boundary layer flow and sheet-flow sand transport.

The insights and parameterizations resulting from this study underline the relevance of progressive wave effects for sand transport and facilitate further development of sand transport formulas used for morphological predictions in engineering practice.

Wouter Kranenburg conducted his PhD research at the Department of Civil Engineering of the University of Twente in the Netherlands.

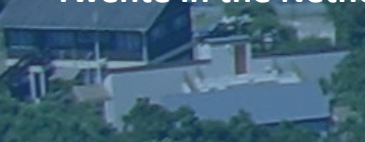

ISBN: 978-90-365-3504-5

DOI: $10.3990 / 1.9789036535045$

University of Twente, The Netherlands
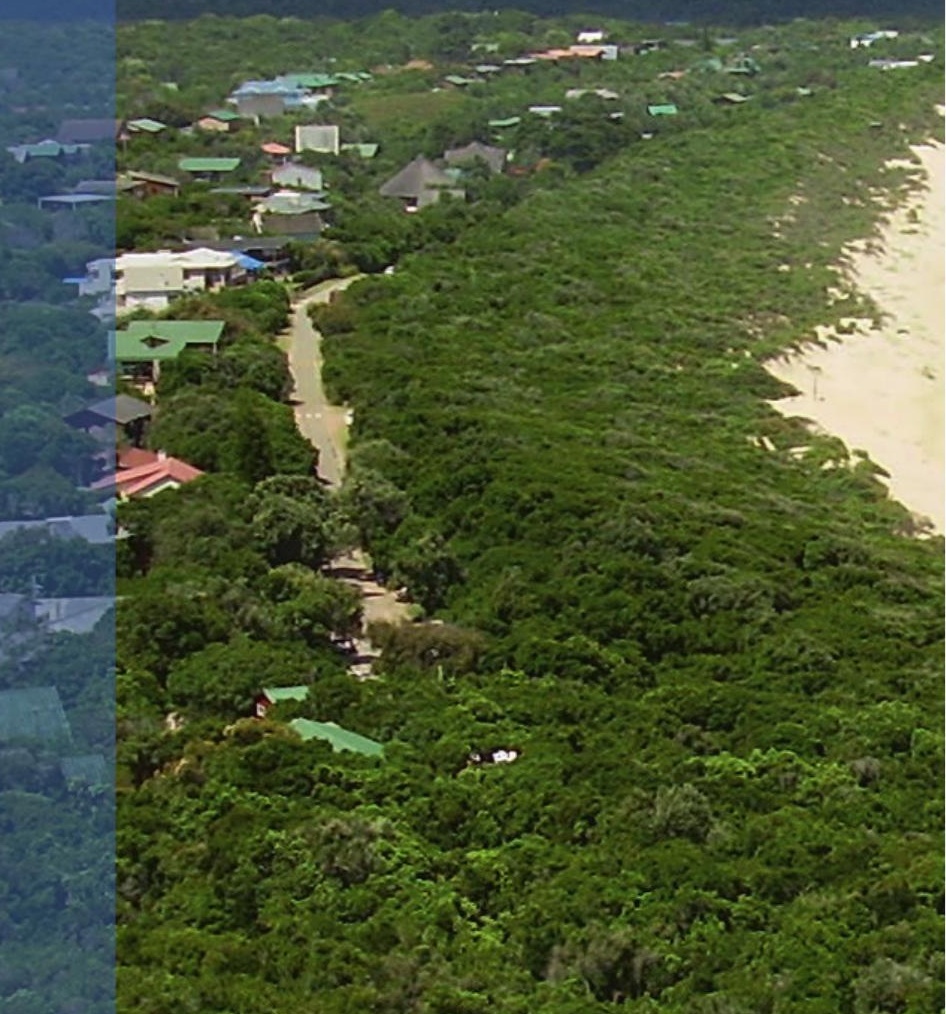